\title{
Bioinspired dinuclear copper complexes for catalytic oxidation of phenolic substrates
}

\author{
Dissertation \\ zur Erlangung des mathematisch-naturwissenschaftlichen \\ Doktorgrades „Doctorum rerum naturalium“ der \\ Georg-August-Universität Göttingen im
}

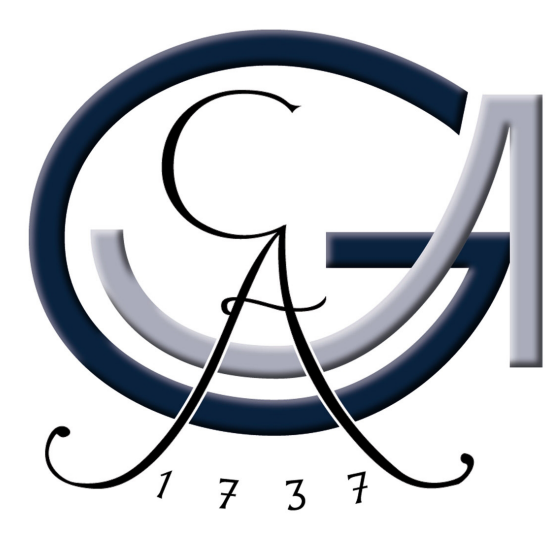

strukturierten Promotionsprogramm Catalysis for Sustainable Synthesis (CaSuS).

\author{
vorgelegt von \\ Marten Seeba \\ aus Upgant-Schott





\section{Betreuungsausschuss}

Prof. Dr. Franc Meyer, Institut für Anorganische Chemie, Georg-August-Universität Göttingen

Prof. Dr. Sven Schneider, Institut für Anorganische Chemie, Georg-August-Universität Göttingen

Prof. Dr. Dietmar Stalke, Institut für Anorganische Chemie, Georg-August-Universität Göttingen

Mitglieder der Prüfungskommission

Referent:

Prof. Dr. Franc Meyer, Institut für Anorganische Chemie, Georg-August-Universität Göttingen

Koreferent:

Prof. Dr. Sven Schneider, Institut für Anorganische Chemie, Georg-August-Universität Göttingen

weitere Mitglieder der Prüfungskommission:

Prof. Dr. Dietmar Stalke, Institut für Anorganische Chemie, Georg-August-Universität Göttingen

Prof. Dr. Manuel Alcarazo, Institut für Organische und Biomolekulare Chemie, Georg-August-Universität Göttingen

Dr. Franziska Thomas, Institut für Organische und Biomolekulare Chemie, GeorgAugust-Universität Göttingen

Prof. Dr. Ricardo Mata, Institut für Physikalische Chemie, Georg-August-Universität Göttingen

Tag der mündlichen Prüfung: 06.11.2017 



\section{Contents}

\begin{tabular}{lll}
\hline $\mathbf{1}$ & Introduction & $\mathbf{1}$
\end{tabular}

1.1 Copper: A versatile protagonist in synthesis and biology . . . . . . . . . . 1

1.2 Copper containing proteins . . . . . . . . . . . . . . . . 2

1.3 Modes of dioxygen binding to two copper(I) ions . . . . . . . . . . . . . 4

$1.3 .1{ }^{\mathrm{T}} \mathbf{P}$ and ${ }^{\mathrm{C}} \mathbf{P}$ binding mode ................. . . . 4

1.4 Binding and activation of dioxygen by dinuclear copper enzymes . . . . . . 6

1.4.1 Oxygen activation and transport by type-3 copper proteins . . . . . 7

1.4 .2 The particulate Methane Monooxygenase (pMMO) . . . . . . . . . 10

1.5 Bioinorganic model complexes . . . . . . . . . . . . . . . 10

1.5.1 Biomimetic dinuclear copper complexes and their reactivity towards endogenous and exogenous substrates . . . . . . . . . . . . . 11

1.5 .2 Self assembly of the copper dioxygen core . . . . . . . . . . . 14

1.5.3 The $\mathrm{O}$ core as an alternative active species . . . . . . . . . . 15

1.6 Binding and activation of sulfur by copper enzymes . . . . . . . . . . . 16

1.7 Homogenous copper catalyzed reactions . . . . . . . . . . . . . . . 16

1.7.1 Oxidative coupling of phenols . . . . . . . . . . . . . 17

$1.7 .2 \mathrm{C}-\mathrm{H}$ bond activation . . . . . . . . . . . . . . 20

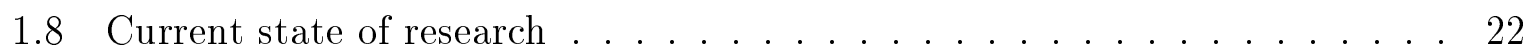

1.8.1 Spectroscopy of copper-phenolate adducts . . . . . . . . . . 26

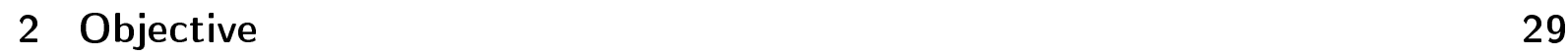

3 Synthesis of pyrazolate based ligand scaffold 31

3.1 Pyrazolate based Ligand Frameworks . . . . . . . . . . . . . . . . . 31

3.2 Synthesis of building blocks . . . . . . . . . . . . . . . . . . . . 33

3.2 .1 Synthesis of the pyrazole backbone . . . . . . . . . . . . . 33

3.2 .2 Synthesis of sidearms . . . . . . . . . . . . . 33

3.3 Synthesis of Ligands $\ldots \ldots \ldots \ldots \ldots \ldots \ldots$

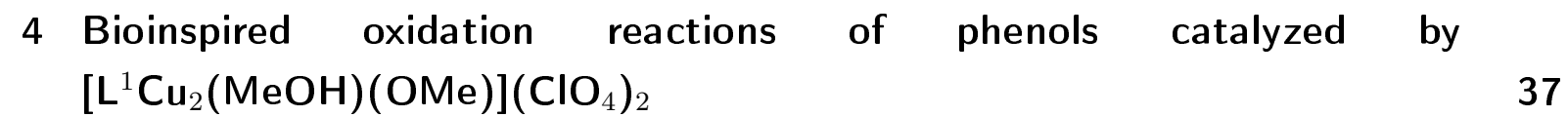

4.1 Mechanistic studies of the oxidation of TMBB . . . . . . . . . . . 37

$4.1 .1 \quad$ Kinetic investigations . . . . . . . . . . . . . . . . . . . 44

4.1 .2 Reoxidation of the mixed valent copper(I)copper(II) species by molecular dioxygen . . . . . . . . . . . . . . . . . . 48 
$4.1 .3 \quad$ Proposed reaction mechanism . . . . . . . . . . . . . . 52

4.2 Functionalization of TMP in para position . . . . . . . . . . . . . 55

4.2 .1 Synthesis of 2,6-dimethyl-4-methylmethoxy-phenol . . . . . . . . . 55

4.2.2 Synthesis of 2,6-dimethyl-4-hydroxymethyl-phenol and subsequent

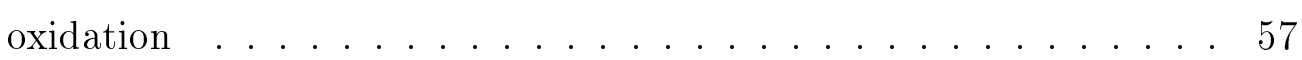

4.2 .3 Direct synthesis of 3,5-dimethyl-4-hydroxy benzaldehyde . . . . . . 64

4.3 Polymerization of 4-bromo-2,6-dimethyl phenol $\ldots \ldots$. . . . . . 70

$4.3 .1 \quad$ Investigation of the mechanism . . . . . . . . . . 70

4.3 .2 Analysis of the obtained polymer and mechanistic considerations. . 74

4.3 .3 Conclusion . . . . . . . . . . . . . . . . . 81

5 Structure and Reactivity of $\mathrm{L}^{2} \mathrm{Cu}_{2}^{\prime \prime}\left(\mathrm{NO}_{3}\right)_{3}$ and $\mathrm{L}^{2} \mathrm{Cu}_{2}^{\prime} \mathrm{OTf}$

5.1 Spectroscopic properties of dicopper complexes of $\mathbf{H L}^{2} \ldots \ldots$. . . . . 83

$5.1 .1 \quad$ Synthesis of the dicopper(I)complex . . . . . . . . . . . . . 86

5.2 Reactivity of the dicopper complexes of $\mathbf{H L}^{2} \ldots \ldots \ldots$. . . . . . . . 89

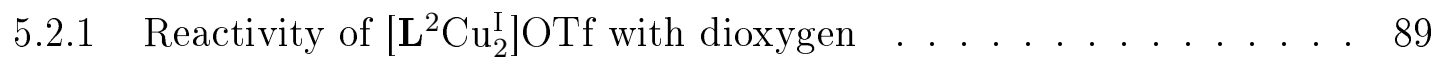

5.2 .2 Reactivity of $\left[\mathrm{L}^{2} \mathrm{Cu}_{2}^{\mathrm{II}}\left(\mathrm{NO}_{3}\right)_{2}\right]\left(\mathrm{NO}_{3}\right)$ with sodium 2,4,6-trimethyl

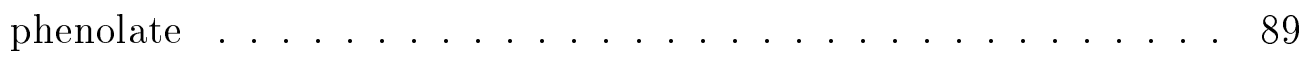

5.2 .3 Reactivity of $\left[\mathrm{L}^{2} \mathrm{Cu}_{2}^{\mathrm{II}}\left(\mathrm{NO}_{3}\right)_{2}\right]\left(\mathrm{NO}_{3}\right)$ with one equivalent of NaTMP. $\quad 90$

5.3 Nature of the intermediate . . . . . . . . . . . . . 98

6 Structure and reactivity of dicopper complexes of $\mathrm{HL}^{3} \quad 105$

6.1 Synthesis and characterization of dicopper(II) complexes of $\mathbf{H L}^{3}$. . . . . 105

6.2 Synthesis, characterization and reactivity of a dicopper(I) complex of $\mathbf{H L}^{3} 107$

6.3 Reactivity with $\mathrm{O}_{2} \ldots \ldots \ldots \ldots \ldots$. . . . . . . . . . . . . . . . . . . . . 108

6.4 Reactivity with Sulfur . . . . . . . . . . . . . . . . . . 109

6.5 Reactivity with $\mathrm{NO}$. . . . . . . . . . . . . . . . . . 112

6.5.1 Reactivity with sodium phenolate . . . . . . . . . . . 117

6.5 .2 Conclusion . . . . . . . . . . . . . . . 118

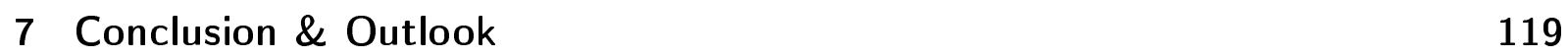

8 Experimental section $\quad 121$

8.1 General considerations . . . . . . . . . . . . . . . . 121

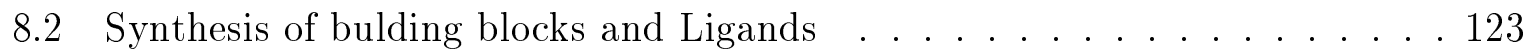

8.2.1 Synthesis of 3,5-Bis(chloromethyl)-1-(tetrahydropyran-2-yl)-pyrazole 123

8.2.2 Synthesis of bis(2-(1-methylbenzimidazolyl)ethyl)amine . . . . . . . 124

8.2.3 Synthesis of Bis(1-methyl-2-phenyl-4-imidazolylmethyl)amine. . . . 124

8.2 .4 Synthesis of $\mathrm{HL}^{1} \ldots \ldots \ldots \ldots \ldots$. . . . . . . . . . . . . 126 
8.2 .5 Synthesis of $\mathrm{HL}^{2} \ldots \ldots \ldots \ldots$. . . . . . . . . . . . . 27

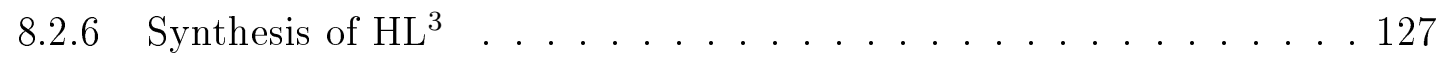

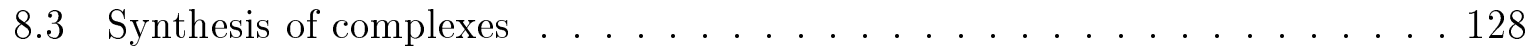

8.3 .1 Synthesis of $\left[\mathrm{L}^{1} \mathrm{Cu}_{2}(\mathrm{OMe})(\mathrm{HOMe})\right]\left(\mathrm{ClO}_{4}\right)_{2}(\mathbf{1}) \ldots \ldots \ldots . .28$

8.3 .2 Synthesis of $\left[\mathrm{L}^{1} \mathrm{Cu}^{\mathrm{II}} \mathrm{Cu}^{1}(\mathrm{MeCN})\left(\mathrm{ClO}_{4}\right)_{2}\right]_{2}(\mathbf{2}) \ldots \ldots \ldots \ldots . .28$

8.3 .3 Synthesis of $\left[\mathrm{L}^{1} \mathrm{Cu}_{2}\left(\mathrm{O}_{2} \mathrm{C}_{9} \mathrm{H}_{9}\right)(\mathrm{HOMe})\right]\left(\mathrm{ClO}_{4}\right)_{2}(\mathbf{3}) \ldots \ldots \ldots . .128$

8.3 .4 Synthesis of $\left.\left[\mathrm{L}^{2} \mathrm{Cu}_{2}\left(\mathrm{NO}_{3}\right)_{2}\right]\left(\mathrm{NO}_{3}\right)(4)\right] \ldots \ldots \ldots \ldots . \ldots \ldots 129$

8.3 .5 Synthesis of $\left.\left[\mathrm{L}^{2} \mathrm{Cu}_{2}\left(\mathrm{H}_{2} \mathrm{O}\right)(\mathrm{OH})\right]\left(\mathrm{NO}_{3}\right)_{2}(5)\right] \ldots \ldots \ldots \ldots$

8.3 .6 Synthesis of $\left.\left[\mathrm{L}^{2} \mathrm{Cu}_{2}\right] \mathrm{OTf}(\mathbf{6})\right] \ldots \ldots \ldots \ldots \ldots \ldots \ldots$

8.3 .7 Synthesis of $\mathrm{L}^{3} \mathrm{Cu}_{2}\left(\mathrm{NO}_{3}\right)_{3}(7) \ldots \ldots \ldots \ldots \ldots$

8.3 .8 Synthesis of $\left.\left[\mathrm{L}^{3} \mathrm{Cu}_{2}(\mathrm{OAc})\right]\left(\mathrm{NO}_{3}\right)_{2}(8)\right] \ldots \ldots \ldots \ldots \ldots$

8.3 .9 Synthesis of $\left.\left[\mathrm{L}^{3} \mathrm{Cu}_{2}\right] \mathrm{OTf}(\mathbf{9})\right] \ldots \ldots \ldots \ldots \ldots$

Overview of complexes . . . . . . . . . . . . . . . . . . . 130

\begin{tabular}{ll}
\hline Bibliography & 131
\end{tabular}

Crystallographic data . . . . . . . . . . . . . . . . . . . 144

Abbreviations . . . . . . . . . . . . . . . . . . . . . . . . 145 



\section{Introduction}

\subsection{Copper: A versatile protagonist in synthesis and biology}

Copper is not only a versatile material for the manufacturing of products for every day use such as pipes, electronic devices and, in the creation of alloys, but also plays a vital role in modern organic synthesis. [1+7]

A large number of copper compounds exist, in which the oxidation state of copper ranges from 0 to $+\mathrm{IV}$. The most common and also most important states are $+\mathrm{I}\left(3 \mathrm{~d}^{10}\right)$ and + II $\left(3 \mathrm{~d}^{9}\right)$, nevertheless $0\left(3 \mathrm{~d}^{10} 4 \mathrm{~s}^{1}\right)$ and + III $\left(3 \mathrm{~d}^{8}\right)$ also play a role in coupling and oxygenation reactions. ${ }^{8[9]} \mathrm{Cu}(\mathrm{I})$ is considered a soft lewis acid and prefers soft lewis bases such as thioethers, phosphanes and unsaturated amines. It is often found in a linear two-coordinate environment but also tolerates up to four-coordinate settings.

$\mathrm{Cu}(\mathrm{II})$ is a moderately hard lewis acid and therefore prefers coordination by harder ligands such as carboxylates, alkoxides, saturated amines and amides. It is usually found four coordinate in a square planar environment or five-coordinate in square pyramidal geometry. The standard aqueous potential for the $\mathrm{Cu}(\mathrm{II}) / \mathrm{Cu}(\mathrm{I})$ redox couple is $0.153 \mathrm{~V}$ vs. NHE. 10111

The standard aqueous reduction potentials show that copper(I) is unstable in aqueous solution. It readily undergoes a disproportionation reaction to form metallic copper $(0)$ and copper(II) $\left(2 \mathrm{Cu}^{+} \rightarrow \mathrm{Cu}^{0}+\mathrm{Cu}^{2+}, \mathrm{E}^{0}=+0.37 \mathrm{~V}\right.$ vs. NHE). Additionally, copper(I) is not stable in aqueous solution under aerobic conditions and oxidized to copper(II). 11 . The variety of oxidation states allows for catalysis tolerating different functional groups through either lewis acidity or $\pi$-coordination. Furthermore, copper can catalyze one electron processes, which are very often of radical nature as well as two electron processes, enabling bond formation reactions similar to those typically mediated by palladium.

A prominent example for lewis acid catalysis is the so called "click-chemistry", a copper(I) catalyzed biocompatible azide-alkyne $[3+2]$ cycloaddtion widely used for tagging biomolecules with sbstrates of choice. ${ }^{8}$ Another example of $\mathrm{C}-\mathrm{C}, \mathrm{C}-\mathrm{O}$ and $\mathrm{C}-\mathrm{N}$ coupling reactions involving copper catalysts are the GLASER-coupling and the ULMANNcoupling. $6[12$

Besides being the protagonist in coupling reactions, copper often enables other catalytic reactions as a co-catalyst, e.g. in the famous STILLE-coupling in which copper(I) acts as an alkyl transfer reagent from stannanes to palladium in a cross metallation reaction or in the widely used WACKER oxidation. 13-15] Therein, two copper(II)chloride units are reduced to copper(I) regenerating the palladium(II) catalyst from its palladium(0) state after a catalytic cycle. Copper(I) is then reoxidized by dioxygen to recover its initial 
copper(II) state. 16$] 17$

Apart from its versatile applicability in organic synthesis, copper is essential in a number of biological processes and occurs in all higher plant and animal species. Embedded into an enzyme, both copper(I) and copper(II) species are stabilized by the provided coordination environment. Due to its ability to easily switch between $\mathrm{Cu}^{+}$and $\mathrm{Cu}^{2+}$ its biological role is focused on electron transport as well as oxygen transport and oxygenation and oxidation reactions. It is found for example in the active center of cytochrome c oxidase, catalyzing the final step in the respiratory chain, which is the simultaneous reduction of dioxygen by four elecrons to two molecules of water. Copper is also found in hemocyanin, an oxygen carrier in molluscs and arthropods and in the particulate methane monooxygenase (pMMO), an enzyme able to oxidize methane to methanol. 10|11|18 20]

All these reactions share the neccessity for the activation of dioxygen to achieve the en-

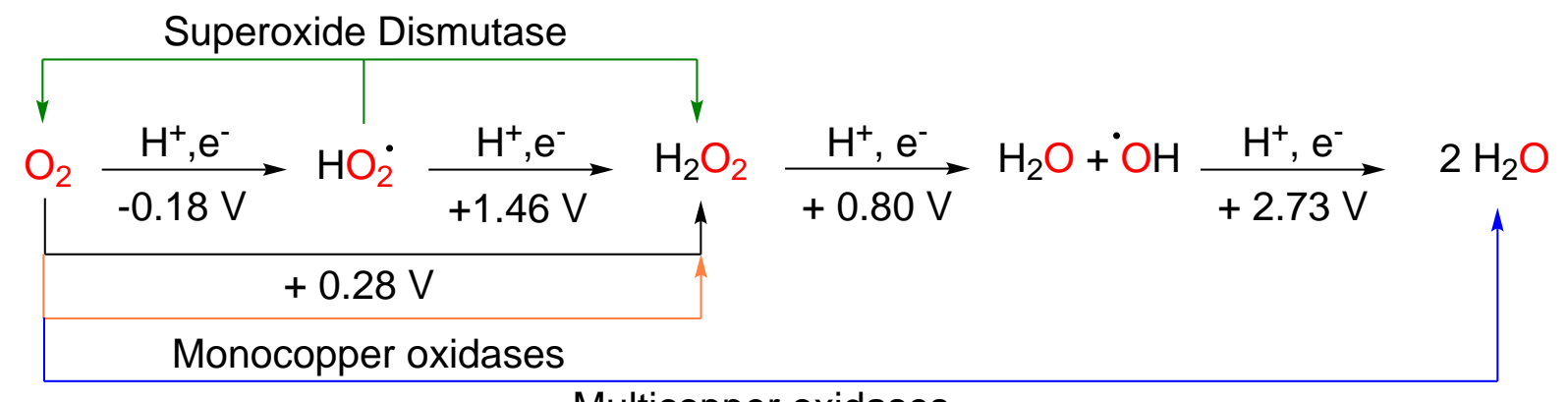

Multicopper oxidases

Figure 1.1 Stepwise $\mathrm{O}_{2}$ reduction and standard potential in aqeous media. 21 Colored arrows show copper enzymes involved in the process. 11122

zymes functionality. Oxygen has a very high activation barrier and therefore does not react with substrates unless a suitable catalyst is employed. The readily switchable copper(I)/copper(II) redox couple exhibits just the right redox potential for the activation of dioxygen $(0.28 \mathrm{~V})$ Figure 1.1). 11122

Being useful in synthetic chemistry and vital in biological reactions it seems selfevident that for the design of catalysts for environmentally benign and sustainable reactions these natural enzymes can serve as blueprints for bioinspired oxidation catalysts. ${ }^{6}$

\subsection{Copper containing proteins}

Originally, copper enzymes have been divided into three groups based on their spectroscopic features. Recent advances, especially in protein crystallography, have led to the distinction of seven different types. 23.

The first one being the mononuclear type-1 active site, also called "blue copper proteins" due to an intense absorption band at around $600 \mathrm{~nm}$ in the oxidized $\mathrm{Cu}$ (II) state, attributed to a $\mathrm{S}$ (cystein) to $\mathrm{Cu}^{\mathrm{II}}$ ligand-to-metal-charge transfer. Their coordination 
environment involves $\mathrm{N}$ and S-donors such as histidine, cysteine and methionin. Later, also green $(450 \mathrm{~nm})$ and red $(390 \mathrm{~nm})$ type-1 active sites were discovered. $24-26$ This class is usually involved in electron transfer processes and found for example in nitrite reductase. 27

The second class comprise mononuclear type-2 active sites, often refered to as normal copper proteins due to their EPR signal similar to low molecular weight $\mathrm{Cu}^{\mathrm{II}}$ complexes $\left(\mathrm{A}_{\mathrm{II}}<140 \cdot 10^{-4} \mathrm{~cm}^{-1}\right)$. $\stackrel{1118}{ }$ Furthermore, they do not exhibit any characteristic intense absorption in this region. Their coordination environment usually involves $\mathrm{N}$ and/or Odonor atoms arranged in either a square planar or distorted tetrahedral geometry. Type-2 centers can be found for example in copper/zinc speroxide dismutase, galactose oxidase 28 and dopamin- $\beta$-hydroxylase ${ }^{28}$. Galactose oxidase is an example for a free radical metalloenzyme active site and dopamin- $\beta$-hydroxylase mediates $\mathrm{C}-\mathrm{H}$ activation in benzylic position for the conversion of dopamin to noradrenaline by hydroxylation. 11123

The type- 3 active site as found in hemocyanin, tyrosinase and catechole oxidase, comprises two copper ions in close proximity, each ligated by three histidine moieties, and is able to reversibly bind dioxygen under ambient conditions. Hemocyanin is responsible for dioxygen transport in arthropods and molluscs. Tyrosinase and chatechole oxidase utilize the bound dioxygen to oxygenate (only tyrosinase) and oxidize phenolic substrates. These enzymes play a vital role in the biosynthesis of melanine which starts with the oxygenation of tyrosine to give L-DOPA which is subsequently oxidized to L-dopaquinone (Figure 1.2. Melanin is a polymeric high-weight pigment involved in colouring of skin and hair, ${ }^{29}$ browning of fruits and vegetables, protection against UV-light, ${ }^{30}$ ionizing radiation, 31 and immunological defense. 32 ]

In type- 4 active sites, a type- 3 and type- 2 center are located in close proximity in a tri-

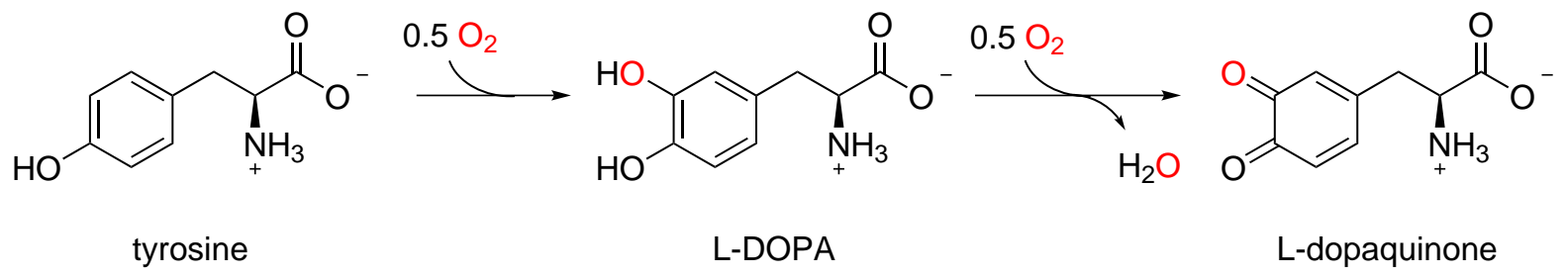

Figure 1.2 Hydroxylation and subsequent oxidation of L-tyrosine as mediated by tyrosinase

angular arrangement.

Furthermore, there is the dinuclear mixed valent $\mathrm{Cu}_{A}$-site, which is also found in cytochrom-c-oxidase, the mononuclear $\mathrm{Cu}_{B}$-site found in heme-copper-oxidase and the tetranuclear $\mathrm{Cu}_{Z}$ center found in $\mathrm{N}_{2} \mathrm{O}$-reductase. 11

Additionally to those naturally occuring sites, a synthetic type-0 active site was synthesized through genetic engeneering of a type-1 site of azurin to study the influence of single 
ligand exchange on its properties. $33 \mid 34$

\subsection{Modes of dioxygen binding to two copper(I) ions}

While there are numerous ways for dioxygen and copper to bind in different stoichiometries, this section will focus on the interaction of one molecule of dioxygen and two copper ions as present in type-3 and type-4 copper enzymes. As shown in Figure 1.3, dioxygen
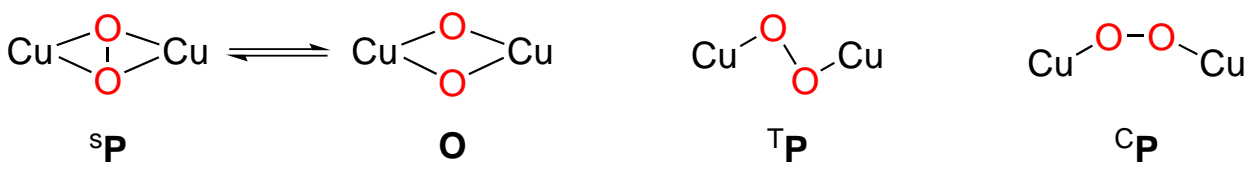

Figure 1.3 Binding modes of dioxygen to copper in a 1:2 ratio.

can bind in at least four different ways between two copper ions. The most commonly found is the side-on peroxo $\mu-\eta^{2}: \eta^{2}$ binding mode $\left({ }^{\mathrm{S}} \mathbf{P}\right)$. ${ }^{35}$ Starting from copper(I), dioxygen is reduced to a peroxo unit and the two copper ions are oxidized to copper(II). It has recently been observed in some synthetic analogues that the ${ }^{\mathrm{S}} \mathbf{P}$ mode can interconvert to the bis- $\mu$-oxo mode $(\mathbf{O})$, wherein the $\mathrm{O}-\mathrm{O}$ bond has been broken by further reduction to the oxo level, while simultaneously oxidizing copper to a + III state. ${ }^{[36}$ With elongated distance between the copper ions, increase in coordination number dioxygen can bind in a $\mu-\eta^{1}: \eta^{1}$ fashion, either in trans $\left({ }^{\mathrm{T}} \mathbf{P}\right)$ or $\operatorname{cis}\left({ }^{\mathrm{C}} \mathbf{P}\right)$ configuration. ${ }^{37 / 38}$ The ${ }^{\mathrm{C}} \mathbf{P}$ mode is regarded as a possible first step in dioxygen binding and investigation of respective complexes helps to understand the interaction of copper and oxygen.

\subsection{1 ${ }^{\mathrm{T}} \mathbf{P}$ and ${ }^{\mathrm{C}} \mathbf{P}$ binding mode}

In 1988, the first X-ray crystal structure of a $\mathrm{Cu}_{2} \mathrm{O}_{2}$ model compound with a ${ }^{\mathrm{T}} \mathbf{P}$ binding mode was reported by KARLIN and ZUBIETA. 37] They employed the tripodal tetradentate ligand tris[(2-pyridyl)methyl]amine (TPA) to form a copper(I) complex, which they reacted with dioxygen at $-80{ }^{\circ} \mathrm{C}$ to reversibly give a trans-peroxo copper complex $\left({ }^{\mathrm{T}} \mathbf{P}\right)$.

Besides the TPA ligand, derivatives of tris(2-aminoethyl)amine (tren) $\stackrel{39400}{3}$ and rac5,5,7,12,12,14-hexamethyl-1,14,8,11-tetraazacylcotetradecane (tetb) 41] (Figure 1.4 have been employed succesfully in the synthesis of ${ }^{\mathrm{T}} \mathbf{P}$ complexes. In case of the tren complexes a two step mechanism in the formation of the ${ }^{\mathrm{T}} \mathbf{P}$ complexes has been observed. ${ }^{39}$ First, an end-on superoxo complex is formed by reaction of $\mathrm{O}_{2}$ with a single copper(I) complex, that rapidly reacts with a second copper(I) unit to form the trans-peroxo complex. Although these species are not considered to be biologically relevant, their investigation 
<smiles>c1ccc(CN(Cc2ccccn2)Cc2ccccn2)nc1</smiles>

TPA<smiles>[R7]CCN(CC[Y7])CCN[R7]</smiles>

$\mathrm{R}^{1}=\mathrm{R}^{2}=\mathrm{Me}=\mathrm{Me}_{6}$ tren $\mathrm{R}_{1}=\mathrm{H}, \mathrm{R}^{2}=\mathrm{Bz}=\mathrm{Bz}_{3}$ tren

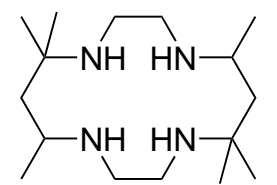

tetb

Figure 1.4 Schematic represenatation of the ligands shown to bin dioxygen in a ${ }^{\mathrm{T}} \mathbf{P}$ manner. $37 / 39.41]$

grants further insight into the binding mechanism of copper and oxygen, and higlights the facts that govern their stability. $\underline{42}$

Just recently, MEYER and coworkers demonstrated the possibility of an end-on cis-peroxo oyxgen binding mode (Figure 1.5). Employing a pyrazolate/tacn system, the first ${ }^{\mathrm{C}} \mathbf{P}$ was crystallographically characterized. ${ }^{38}$ The $\mathrm{Cu}-\mathrm{O}-\mathrm{O}-\mathrm{Cu}$ torsion angle is $65^{\circ}$ and leads to a reduced overlap in magnetic orbitals, leading to different magnetic properties, while the remaining spectral data does not change significantly. One year after, the first ferromagnetically coupled dicopper(II) peroxo system with a triplet ground state $(S=1)$ was reported by the same group. $\frac{43}{3}$ Through elongation of the ligand backbone, it was possible to decrease the $\mathrm{Cu} \cdots \mathrm{Cu}$ distance and hence to increase the $\mathrm{Cu}-\mathrm{O}-\mathrm{O}-\mathrm{Cu}$ torsion angle to $104^{\circ}$ which is close to a $90^{\circ}$ angle. Orthogonality of the involved orbitals leads to ferromagnetic coupling. This system can be considered a snapshot into the first step of dioxygen binding in type-3 copper proteins.

The first crystal structure of an ${ }^{\mathrm{S}} \mathbf{P}$ adduct was published by KITAJIMA in 1989 making
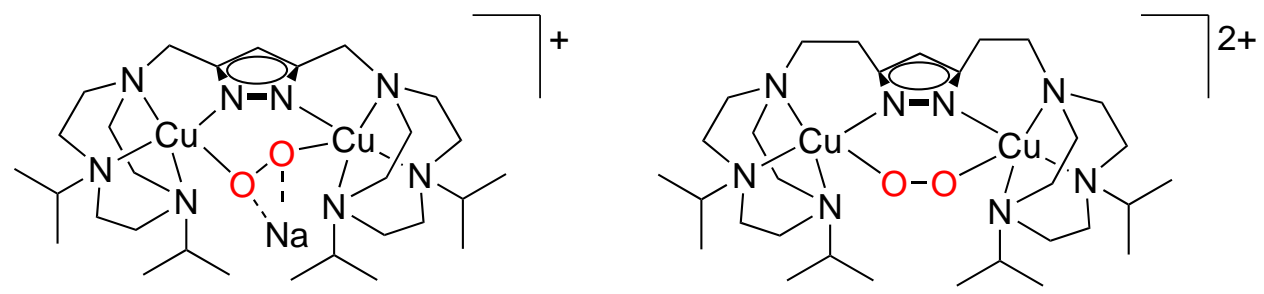

Figure 1.5 left dinculear coppeer(II) ${ }^{\mathrm{C}} \mathbf{P}$-complex. right)dinculear copp(II) ${ }^{\mathrm{C}} \mathbf{P}$-complex with an $S=0$ ground state.

use of the tridentate tris(3,5-di-iso-propyl-pyrazolyl) borate ligand. ${ }^{44}$ A variety of ${ }^{\mathrm{S}} \mathbf{P}$ complexes were found to exist before this motif was disvovered to represent also the binding motif in oxy-hemocyanin. 35 


\subsection{Binding and activation of dioxygen by dinuclear copper en- zymes}

In order to sustain their energy demand, organisms can process their aquired nutrients in either an anaerobic or an aerobic manner. Aerobic respiration is the most exergonic metabolic pathway known and therefore delivers more energy than anaerobic metabolism. In consequence, where dioxygen is available this pathway is favored. Today, dioxygen makes up about $20 \%$ of the earth's atmosphere. It is found in a triplet ground state $\left({ }^{3} \mathrm{O}_{2}\right)$ with two unpaired electrons, one in each of the doubly degenerate $\pi^{*}$ HOMOs. Although $\mathrm{O}_{2}$ has a high oxidation potential, the reaction with singlet molecules, such as organic substrates, requires a quantummechanically forbidden spin conversion and therefore exhibits a high kinetic barrier. To overcome this spin restriction, proteins have redox active metals in their active center. Iron and copper are natures metals of choice for this purpose.

Copper-containing enzymes make use of the thermodynamically favourable reduction of

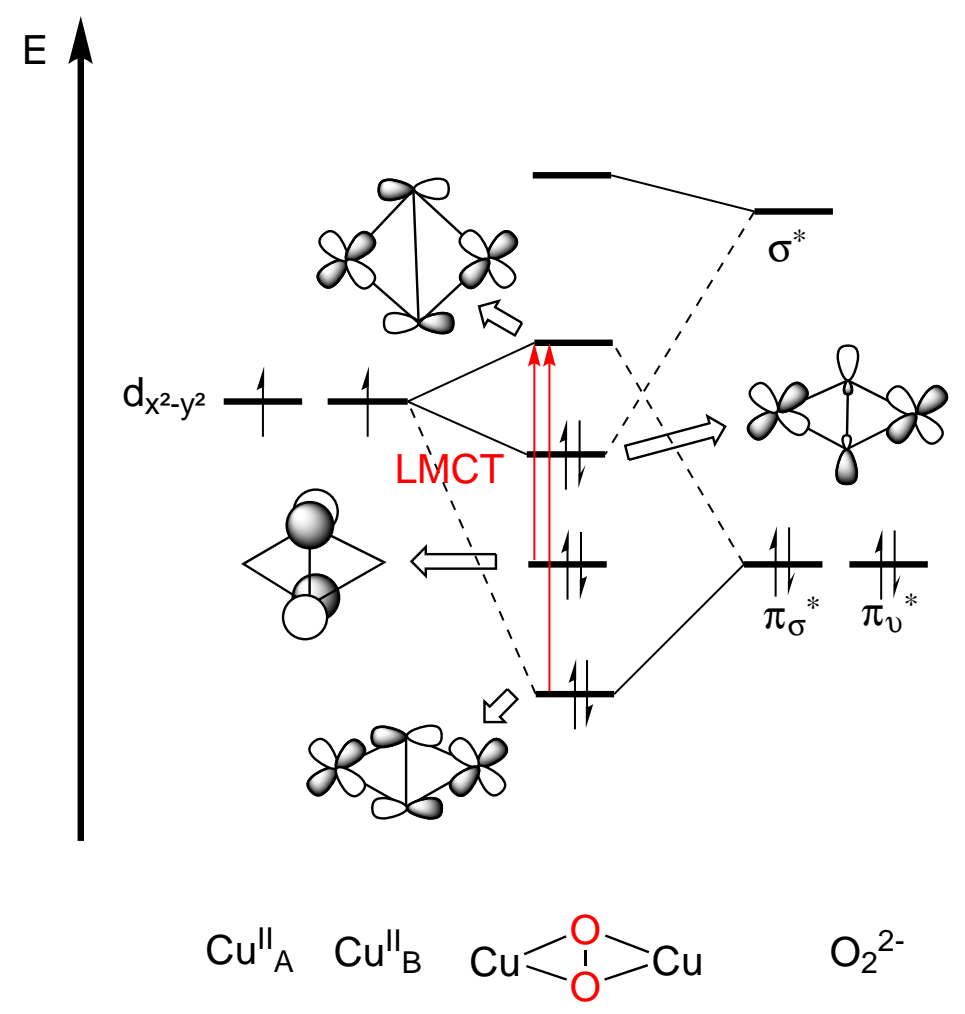

Scheme 1.1 Schematic MO diagram of a side-on $\mu-\eta^{2}: \eta^{2}$ dicopper(II) peroxo species. $\frac{9142}{2}$

$\mathrm{O}_{2}$ to peroxide $\mathrm{O}_{2}^{2-}$, which leads to an elongation of the $\mathrm{O}-\mathrm{O}$ bond, concomittant with a decrease in the $\tilde{\nu}(\mathrm{O}-\mathrm{O})$ stretching frequency and can lead to bond breaking as the aforementioned antibonding orbitals are filled Scheme 1.1, 11]

In order to transport and bind $\mathrm{O}_{2}$, it is usually reduced by two electrons to the peroxo 
level. For the activation of $\mathrm{O}_{2}$ that involves a complete breakage of the $\mathrm{O}-\mathrm{O}$ bond, four electrons are required in total. If dioxygen is used as an electron acceptor, additional two protons are needed to release hydrogen peroxide in the case of mononuclear and additional four protons to release water in the case of di- and multinuclear enzymes. ${ }^{45}$

\subsubsection{Oxygen activation and transport by type-3 copper proteins}

Most animals rely on circulary transport of dioxygen, which they need for their metabolism, and have specific proteins designated for this task. Apart from the ironcontaining hemoglobin (found in all mammals) and the non-heme hemerythrin (found in marine invertebrates), an enzyme containing a coupled dinuclear copper core called hemocyanine is found freely dissolved in the blood of molluscs and arthropods. ${ }^{46}$

This dinuclear type-3 active site is also found in tyrosinase and catechole oxidase, which belong to the class of polyphenol oxidases (PPOs). ${ }^{46}$ Furthermore, tyrosinase is able to both oxygenate monophenolic substrates (monophenolase activity) and oxidize ortho-diphenols (diphenolase activity), while catechol oxidase only exhibits the latter activity. Although their primary and tertiary structutre is very dfferent, the active center of all three proteins exhibits a very similar motif. The center is enclosed in four $\alpha$-helices that provide each copper(I) ion with a threefold N-histidine coordination in a trigonal planar fashion. These two central ions are distinct as $\mathrm{CuA}$ and $\mathrm{CuB}$ (see Figure 1.6). There has been much discussion in the literature whether $\mathrm{CuA}$ or $\mathrm{CuB}$ is the binding site for substrates or whether this dependeds on the substrate. To date, due to crystallograhpic data available and computational models, it is commonly believed that $\mathrm{CuA}$ is the binding site for all substrates. ${ }^{47}$

These enzymes make use of the easily accessible copper(I)/copper(II) redox couple to reversibly bind dioxygen under ambient conditions. From the dicopper(I) state (deoxy-form), which does not exhibit any charactersitic spectroscopic signals, oxygen is reduced to a peroxo state and bound in a $\mu-\eta^{2}: \eta^{2}$ fashion to two oxidized copper(II) ions (oxy-form) which gives rise to absorption bands at around $330 \mathrm{~nm}\left(\epsilon \sim 20 \mathrm{mM}^{-1}\right)$ and around $550 \mathrm{~nm}\left(\epsilon \sim 1 \mathrm{mM}^{-1}\right)$. $\stackrel{42}{ }$ Despite the $\mathrm{d}^{9}$-configuration of copper(II), the oxy-form is EPR-silent, due to antiferromagnetic coupling thorugh the bound oxygen. The two copper(I)-ions are acting cooperatively and are simultaneously oxidized by one electron per metal, to give two copper(II)-ions. Upon release of dioxygen the process is reversed. 23

To prevent substrate oxidation in the transport protein hemocyanin the active site is blocked by a phenyl alanine moiety with the phenyl ring shielding the binding pocket and preventing potential substrates from entering the active center. ${ }^{49}$. When this phenyl ring is protoelytically cleaved, hemocyanin can exhibit diphenolase activity. $\frac{50}{[}$ 


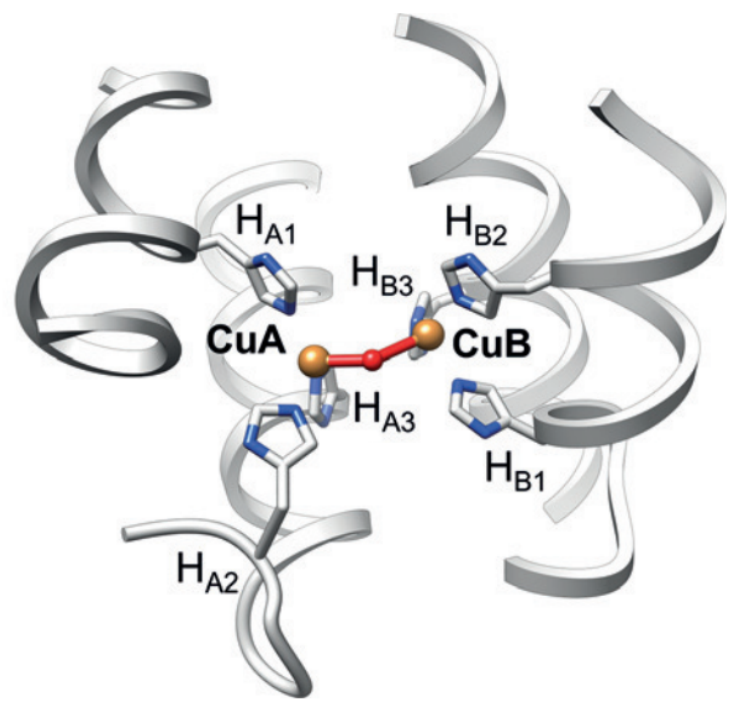

Figure 1.6 Type-3 copper center of VvPPOg from wine grapes in its resting state. 48

While tyrosinase is able to hydroxylate monophenols to give $o$-diphenols and oxidize $o$-diphenols to $o$-quinones, catechole oxidase only catalyzes the oxidation of $o$-diphenols to $o$-quinones. Earlier explanations assumed this difference was due to a shielding of the $\mathrm{CuA}$ site, which is assumed to be the binding site for the hydroxo group of the monophenol, by residues such as phenylalanin, leucine or tyrosine. ${ }^{50}$ Later it was found that this shielding moiety rather acts as a gate residue that can be found in catechole oxidases as well as in tyrosinases and does not inhibit but regulate substrate access

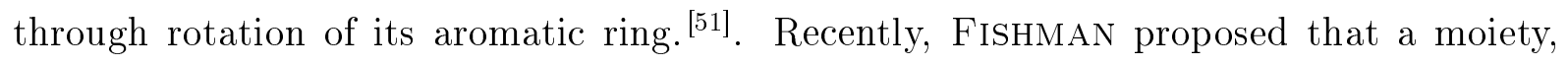
conserved in all known tyrosinases, consisting of a glutamic acid and aspartic acid residue and a water/hydoxide molecule in close proximity to the active site served as an internal base for phenol deprotonation which is a neccessary step in order for the phenol to bind to the $\mathrm{CuA}$ site. This assumption was also verified experimentally through directed mutations. The absence of these residues in catechole oxidase also explains their lack of monophenolase activity. $47 / 48$

Calculations showed that hxydroxylation most likely occurs via electrophilic attack of the bound peroxide to the aromatic ring, hydroxylating the substrate while simultaneously breaking the $\mathrm{O}-\mathrm{O}$ bond, resulting in an asymmetric binding of the formed diphenol and an uptake of the substituted proton by the remaing oxygen atom to form a hydroxide. 52 ] In this scenario, the bound substrate's aromatic ring is oriented parallel to a histidine residue via hydrophobic $\pi-\pi$ interaction, while a butterfly distortion of the $\mathrm{Cu}_{2} \mathrm{O}_{2}$ core occurs which enforces substrate reorientation towards the core and results in hydroxylation of the aromatic ring. Important for the conversion rate and therefore the enzymes activity seem to be the already mentioned gate residue as well as a thioether bond to the second histidine coordinating $\mathrm{CuA}$ that limits its flexibility, which was found 


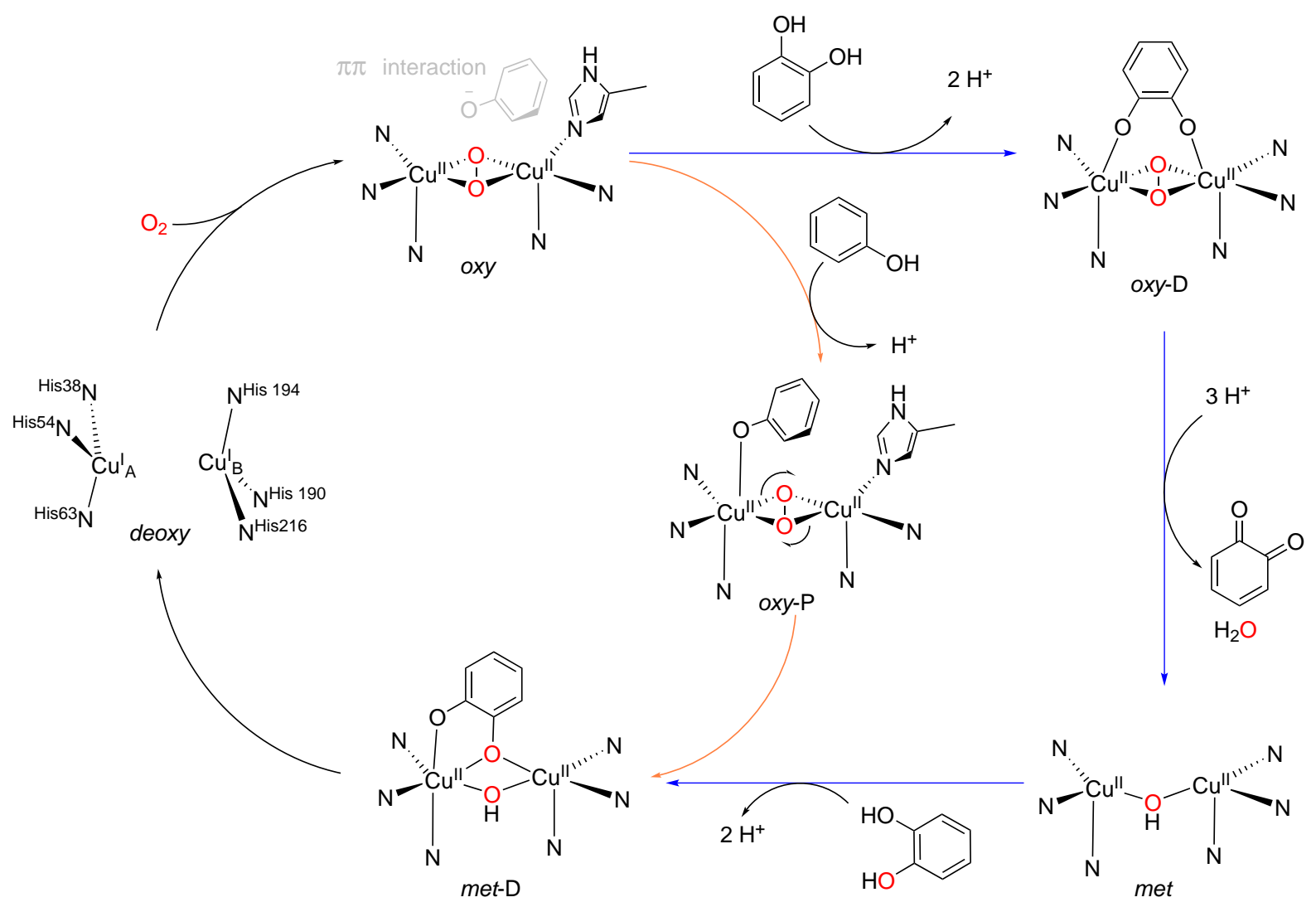

Figure 1.7 Proposed mechanistic cycle of tyrosine hydroxylation.

to be important for catalysis and to achieve substrate specificity.

The catalytic cycle of tyrosinase (Figure 1.7) comprises four distinct oxidation states. 53 , Tyrosinase is mostly found in its resting state, the met-form, with two copper (II) ions that are hydroxide-bridged and found $2.90 \AA$ apart. Phenols can reversibly bind to the core in this state but are not oxidized. Catechols however are oxidized to o-quinones and the copper ions reduced to copper(I), the so called deoxy-form, with the ions being 4.40 $\AA$ away from each other. This state readily reacts with dioxygen to give the oxy-state in which the copper ions are found in a + II state and are bridged by a peroxide in a $\mu-\eta^{2}: \eta^{2}$ fashion. The metal-metal separation is reduced to $3.50 \AA$ by relocation of the copper ions while the coordinating histidine residues remain unchanged. $54 \mid 55$.

From the oxy state phenols and catecholes can be oxidized. The oxidation of phenols involves a monooxygenase reaction and leads back to the deoxy-state, while the reaction with catechols leads to the met-form. The fourth state is the result of the so called "suicide deactivation" in which a catechole is treated as a phenol by oxy-tyrosinase leading to a copper(0) copper(II) state, the so called deact-tyrosinase. 56 


\subsubsection{The particulate Methane Monooxygenase (pMMO)}

Besides the iron-containing soluble methane monooxygenase (sMMO) 57 a copper based trans-membrane counterpart exists, namely the particulate methane monooxygenase (pMMO), that also is capable of oxygenating methane. 58. Since it is a membrane bound protein, characterizing the pMMO is a very challenging task. For this reason, although a crystal structure was available ${ }^{59}$, the actual metal composition as well as the location of the active center remained unknown, until in 2010 ROSENZWEIG and coworkers found a dicopper center in the active site. ${ }^{1959}$ EPR-investigations of the soluble pmoB
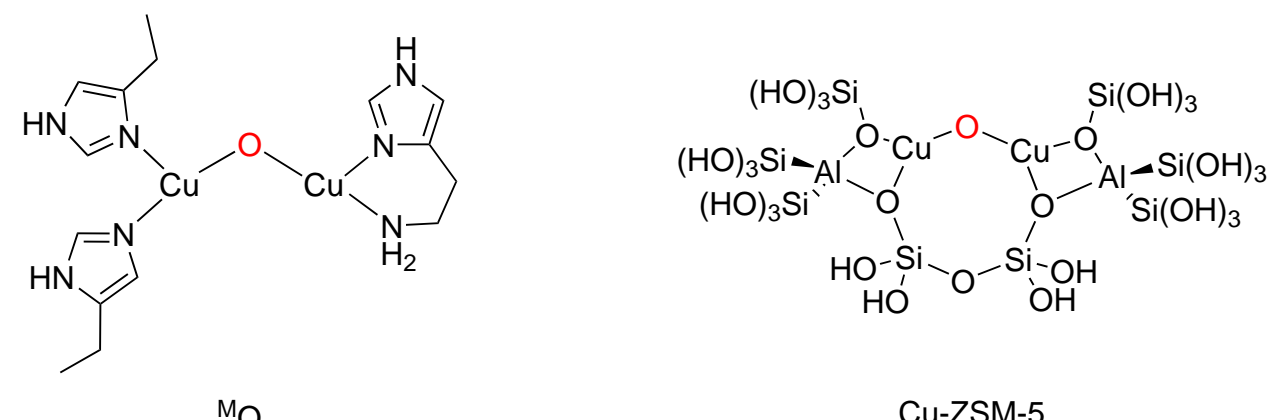

${ }^{\mathrm{M} O}$

Cu-ZSM-5

Figure 1.8 Postulated active species of pMMO and inorganic model compound Cu-ZSM5.

subunit of pMMO revealed that the dinuclear center is found in a valence scrambled copper(I)copper(II) state while a distant mononuclear site, not conserved in all pMMOs, was assigned a copper(I) state. ${ }^{60}$ The actual catalytic cycle is still under debate, but dioxygen binding was found to occur in ${ }^{\mathrm{S}} \mathbf{P}$ mode. Upon exposure to methane its spectroscopic features disappeared, suggesting its involvement in the oxidation process. $\frac{61}{6}$ However, a $\mu$-oxo core is suggested to be the active species responsible for the oxygenation of methane. In 2009, SolOmon found the copper loaded aluminosilicate zeolite Cu-ZSM-5 that also oxidizes methane to methanol to be most similar to pMMO. ${ }^{62}$ This zeolite contains a dicopper(II) $\mu$-oxo core, lending further support to the assumption that $\mathrm{O}_{2}$-activation occurs in the dicopper center in pMMO.

\subsection{Bioinorganic model complexes}

Model complexes of metalloenzymes are synthetic, low molecular weight analogues of the active site of the parent enzyme. These mimics help to gain insight into the geometric and electronic structure of the enzyme's active site and provide reference for its spectroscopic features. Additionally, they help to understand the role of metals in biology and the reaction pathway a substrate undergoes. These biomimetic and/or bioinspired complexes provide helpful insight to develop new catalysts for selective oxidation reactions under 
mild and environmentally benign conditions. Especially the development of oxidation catalysts using dioxygen as the oxidant are of interest from an economical perspective. In the majority of industrial oxidation processes, a stoichiometric amount of metal based oxidant is needed, resulting in a large scale of toxic metal waste. ${ }^{63}$ Biomimetic/bioinspired copper systems are attractive catalysts for these reactions, as copper is abundant, nontoxic and relatively cheap, compared to many other transition metals. Recently achieved reactions include the catalytic oxidation of a range of alcohol to the corresponding aldehydes, ${ }^{64] 65}$ oxidation of benzene to phenol in the presence of hydrogenperoxide, ${ }^{66}$ catalytic conversion of benzylic alchols to the corresponding aldehydes and nitriles (in the presence of ammonia), ${ }^{67}$ cleavage of $\alpha-\mathrm{C}(\mathrm{O})-\mathrm{C}$ bonds to generate acids, ${ }^{68}$ synthesis of oxindoles by catalytic aerobic functionalization of phenols, $\frac{69}{2}$ aromatic $\mathrm{C}-\mathrm{H}$ alkoxylation, ${ }^{70}$ as well as the oxidation of monophenols to ortho-quinones. 71

\subsubsection{Biomimetic dinuclear copper complexes and their reactivity towards endogenous and exogenous substrates}

The regiospecific ortho-hydroxylation as performed by tyrosinase is a difficult task to perform under laboratory conditions and requires multiple steps, sometimes expensive transition metal catalysis, and often only affords low yields. ${ }^{72+76}$ Altough a lot of research has been undertaken in this field, only a few examples of stoichiometric ${ }^{\text {77. } 779}$ and catalytic $[80181$ ortho-phenol hydroxylation reactions are known to date. One of the first examples of a dinuclear copper complex to bind dioxygen and exhibit monooxygenase activity was published by KARLIN and coworkers in 1984. ${ }^{[2]}$ Complex I was found to react

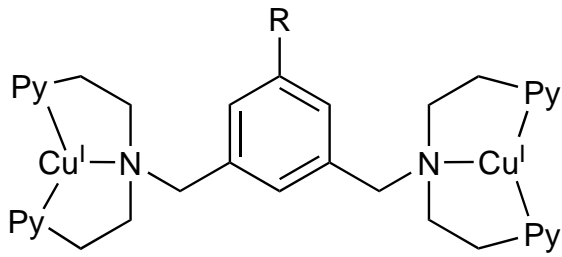

TMP1

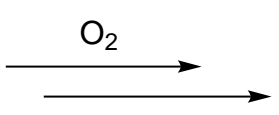

TMP2

Py $=2$-Pyridyl

$\mathrm{R}=\mathrm{H}, \mathrm{NO}_{2}, \mathrm{C}\left(\mathrm{CH}_{3}\right)_{3}, \mathrm{~F}$

Scheme 1.2 Intramolecular hydroxylation in a dinuclear copper complex.

with dioxygen at low temperatures to form a side-on peroxo intermediate that, after rotation of the xylene ring, undergoes a nucleophilic attack by the bound peroxide and results in a hydroxylated aromatic ring, $\mathrm{O}-\mathrm{O}$ bond cleavage and a remaining bridging hydroxide 
bound to two copper(II) ions (II) Scheme 1.2). These findings inspired further studies on ligand hydoxylation. When KARLIN and coworkers employed an already hydroxylated ligand under the same conditions, they found a second hydroxylation of the ligand in para-position. Additionally, further reaction did not lead to the expected oxidation to the para-quinone but rather to arylation of one of the hydroxy-functions. 83 .

TUCZEK et al. recently reported the syntheses of two ligands with an appended phenol<smiles>Oc1cccc(CN(Cc2ccccn2)Cc2ccccn2)c1</smiles>

L4-H<smiles>Oc1cccc(CN(CCc2ccccn2)CCc2ccccn2)c1</smiles>

L5-H

Figure 1.9 Ligands employed by TUCZEK and coworkers to investigate ligand hydroxylation. 84185

close to the metal (Figure 1.9). The copper(I) complex of $\mathbf{L} \mathbf{4}-\mathbf{H}$ forms an ${ }^{\mathrm{S}} \mathbf{P}$-system at $-78^{\circ} \mathrm{C}$ and gave the $o$-quinone without additional base upon warming to room temperature. The ${ }^{\mathrm{S}} \mathbf{P}$-complex of $\mathbf{L} \mathbf{5}-\mathbf{H}$ lead to N-dealkylation and the formation of metahydroxybenzaldehyde. 84185 .

In 1991, CASELLA reported the synthesis of L66 using the same $m$-xylene spacer as KARLin but with $N$-methyl-benzimidazole sidearms. They observed regiospecific ortho-

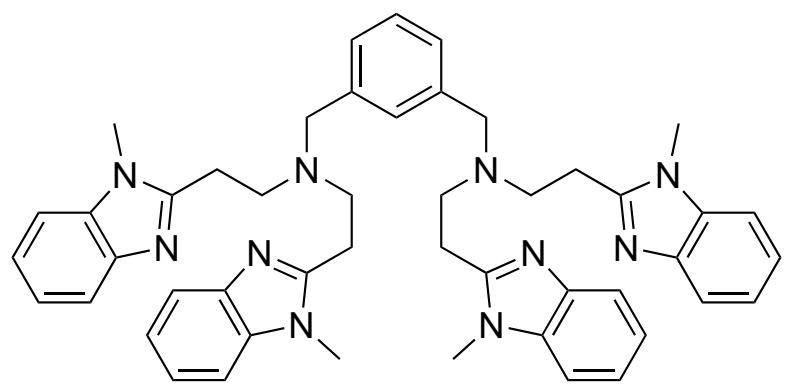

L66

Figure 1.10 Xylene bridged dinucleating amine ligand L66, synthesized by CASELLA and corworkers. 86

hydroxylation towards exogeneous subtrates (Figure 1.11). Complex III forms the stable dioxygen adduct $\mathbf{I V}$ at $-78^{\circ} \mathrm{C}$ in acetone. The reactivity studies were carried out in acetonitrile with prior addition of the substrate and subsequent introduction of dioxygen to the system. The electron poor 4-carboxymethyl phenol was hydroxylated to the cor- 


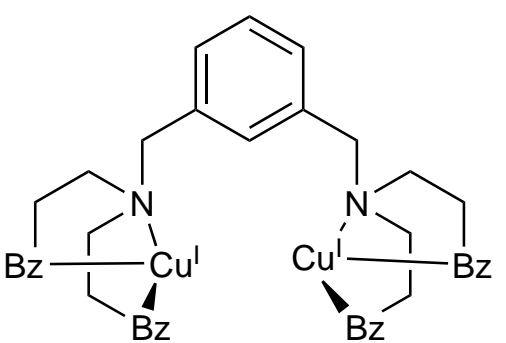

TMP1

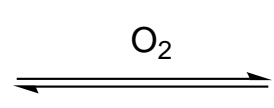

\section{$\mathrm{Bz}=\mathrm{N}$-methyl benzimidazole}

Figure 1.11 reversible bonding of dioxygen at $-80 \mathrm{C}$ by complex

responding catechole and the electron rich 3,5-di-tert-butylcatechole was oxidized to the corresponding quinone. A turnover number (TON) of 1.2 was achieved using the electron rich 2,4-di-tert-butylphenol (DTBP) as a substrate. ${ }^{[8] 86}$ - 88 Around the same time, in 1990, RÉGLIER reported a dicopper system with a biphenyl spacer and pyridylethylimine sidearms. Reaction of the resulting copper-dioxygen adduct with DTBP gave the 6,6' $\mathrm{C}-\mathrm{C}$ coupled dimer in the absence of base and the fully oxidized 3,5-di-tert-butyl quinone (DTBQ) in the presence of triethylamine with a TON of 16. ${ }^{89}$

Two decades later, in 2010, TUCZEK and coworkers developed mononuclear [2(aryl)ethyl]imino-tert-butyl ligands that can be considered sidearms of the RÉGLIER system without the spacer. Very similar to what had been observed in the dinuclear system, a TON of 18 was achieved for the conversion of DTBP to DTBQ in presence of $\mathrm{NEt}_{3}$ when using the 2-pyridyl containing ligand. 90 The highest TON of 31 was achieved with the N-methyl-benzimidazole ligand. ${ }^{91192}$

In these systems, only in the case presented by CASELLA a $\mathrm{Cu} / \mathrm{O}_{2}$ intermediate could

a)

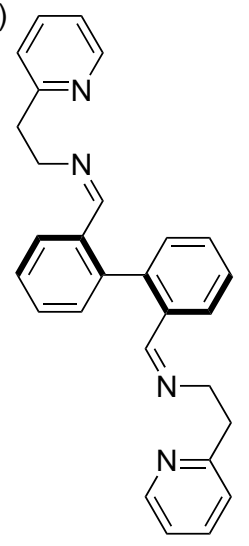

b)

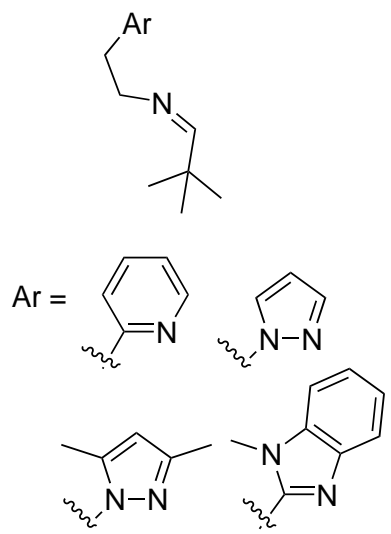

c)

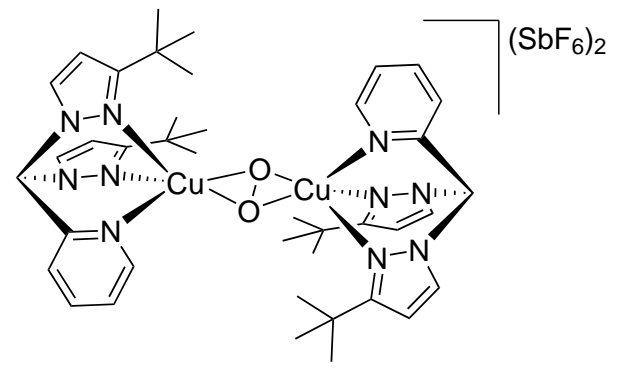

Figure 1.12 a) $\mathrm{BiPh}(\text { impy)})_{2}$ ligand by RÉGLIER, b)mononucleating ligands by TuCzEK c) newly designed ${ }^{\mathrm{S}} \mathbf{P}$ complex by Herres-Pawlis and Stack. 
be identified as the active species. In 2013, HERRES-PAWLIS and STACK developed a new biomimetic $\mu-\eta^{2}: \eta^{2}$ dicopper(II)-peroxo-system ${ }^{80}$ based on a bis(pyrazolyl)(2pyridyl)methane mononucleating ligand. ${ }^{93}$ Upon exposure of the monomeric $\left[\mathrm{Cu}^{\mathrm{I}}\left\{\mathrm{bis}\left(3^{\prime}-\right.\right.\right.$ tert-butyl-pyrazolyl)pyridyl-methane $\}] \mathrm{SbF}_{6}$ to $\mathrm{O}_{2}$ in DCM, the dimeric $\mathrm{Cu}_{2} \mathrm{O}_{2}$ complex (Figure 1.12c) was formed and identified by means of UV/Vis, resonance Raman spectroscopy, and ESI-TOF cryo mass spectrometry. Unexpectedly, the complex is relatively stable at room temperature and decays with a half-life of $30 \mathrm{~min}$. This complex is able to stoichiometrically oxygenate a variety of para-substituted phenolates to the respective catecholates at $-78^{\circ} \mathrm{C}$. This reaction most likely follows an electrophilic aromatic substitution mechanism like the reaction catalyzed by the parent enzyme. Furthermore, catalytic conversion of phenols to quinones was achieved at room temperature in the presence of $\mathrm{NEt}_{3}$ with a TON of up to 15 .

\subsubsection{Self assembly of the copper dioxygen core}

Similar to the threefold coordination of copper found in type-3 enzymes, most ligands employed in model mono- and dinuclear complexes mimicking the $\mathrm{Cu}_{2} \mathrm{O}_{2}$ core share this tridentate binding motif. Almost all of these complexes are only stable at low temperatures around $-80^{\circ} \mathrm{C}$. In solution, the $\mathrm{Cu}_{2} \mathrm{O}_{2}$ core is formed in a self-assembly process by two mononuclear copper(I) complexes and one molecule $\mathrm{O}_{2}$. Besides this process, self assembly is found in a variety of biologic systems, such as lipid bilayers, (desoxy-)ribonucleic acid, protein folding, viruses like the tobacco mosaic virus and the iron-sulphur clusters in proteins. Researchers hence try to implement self assembly into applicable chemistry like template synthesis and host-guest interaction in supramolecular chemistry. ${ }^{94}$.96 Recently, STACK and coworkers were able to show that ${ }^{\mathrm{S}} \mathbf{P}$ complexes analogous to oxy-tyrosinase form in a self assembly process. A $\mathrm{Cu}_{2} \mathrm{O}_{2}$-core was formed, when $\left[\mathrm{Cu}^{\mathrm{I}}(\mathrm{MeCN})_{4}\right] \mathrm{SbF}_{6}$, (2-methyl-)imidazole and $\mathrm{O}_{2}$ were dissolved in 2-methyltetrahydrofurane (MeTHF) at $-125^{\circ} \mathrm{C}$ Scheme 1.3. ${ }^{177}$ The low temperature is required

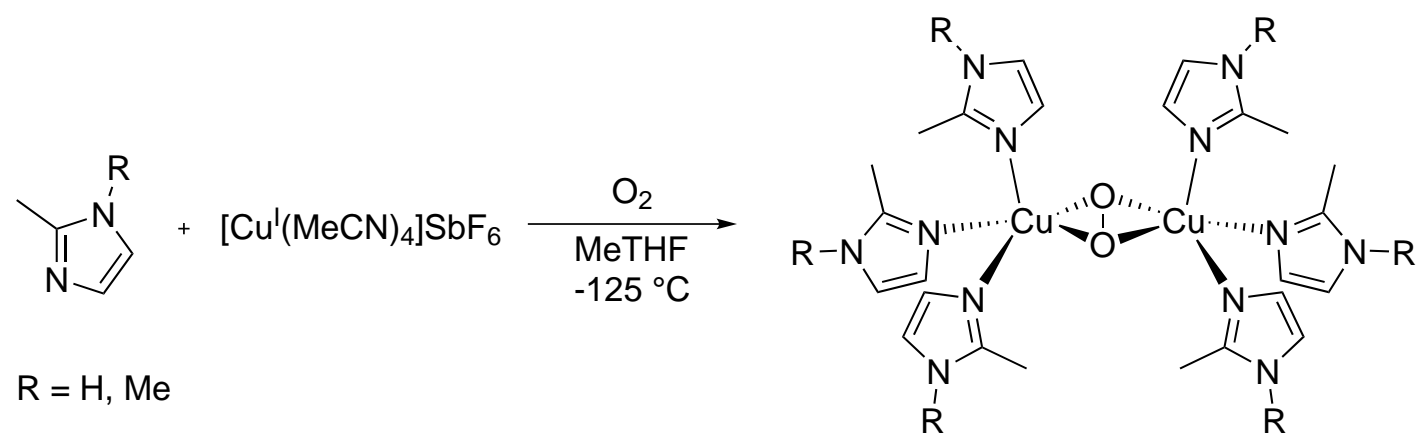

Scheme 1.3 Self assembly reaction of the $\mathrm{Cu}_{2} \mathrm{O}_{2}$-core. 77 .

to compensate for the entropic loss ocurring due to the association. These ${ }^{\mathrm{S}} \mathbf{P}$ complexes 
succesfully performed the electrophilic aromatic oxidation of exogenous phenolic substrates to catecholes, suggesting that the protein matrix in the enzyme is mainly needed to prevent oxidative degradation and overcome the entropic costs of self-assembly.

\subsubsection{The $\mathrm{O}$ core as an alternative active species}

As mentioned above, there is experimental evidence that an aromatic electrophilic substitution mechanism by the ${ }^{\mathrm{S}} \mathbf{P}-\mathrm{Cu}_{2} \mathrm{O}_{2}$ core is the main step in the hydoxylation of phenols. This was confirmed in both model- and enzymatic systems. There is, however, some ambiguity concerning the nature of the $\mathrm{Cu}_{2} \mathrm{O}_{2}$-core, as there is an equilibrium between the ${ }^{\mathrm{S}} \mathbf{P}$ and the $\mathbf{O}$ mode in some synthetic compounds. The $\mathbf{O}$ mode should therefore be considered as a possible active species in the hydroxylation of phenols.

STACK and coworkers found that during a monooxygenation reaction an ${ }^{\mathrm{S}} \mathbf{P}$ species
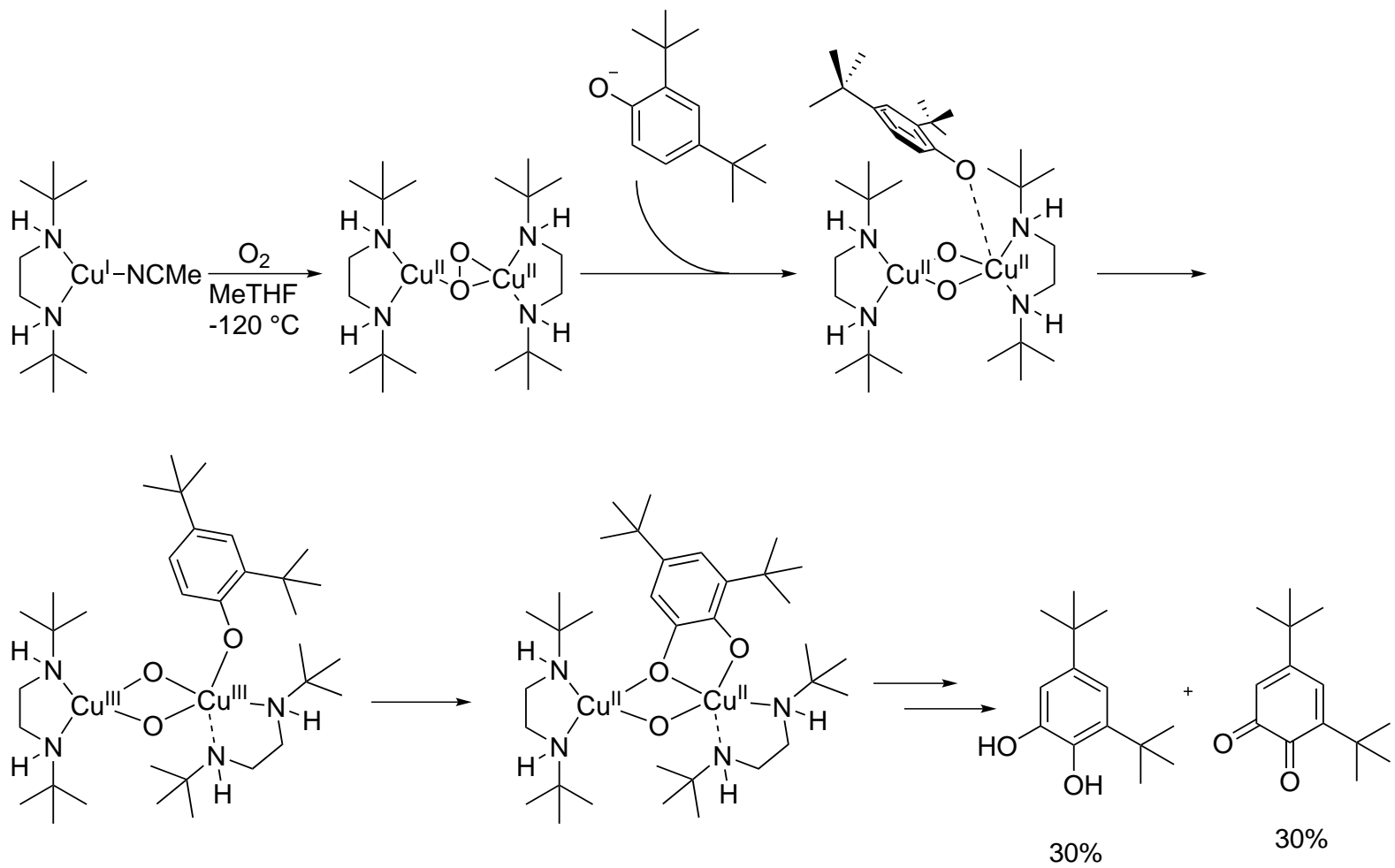

Scheme 1.4 Tyrosinase reactivity in a model complex

coordinated by a secondary amine ${ }^{97 / 98}$ vanished and formed the respective $\mathbf{O}$ isomer (Scheme 1.4. [99] By resonance Raman spectroscopy, O-O bond scission was observed upon substrate coordination at $-120^{\circ} \mathrm{C}$ in 2-methyl tetrahydrofurane. Additionally to these low temperatures, the sterically demanding N,N'-di-tert-butylethylene diamine (DBED) ligand was neccessary to stabilize the intermediate. Upon warming of the mixture, the substrate DTBP was hydroxylated to the respective catechole $(30 \%)$ and oxidized to the quinone $(30 \%)$ by the $\mathbf{O}$ core in an electrophilic aromatic substitution mechanism. 99100 
DFT calculations revealed formation of an intial axial bond of the phenolate to a copper(III) ion and subsequent rotation into the equatorial plane of the $\mathrm{Cu}_{2} \mathrm{O}_{2}$ core, where the newly formed $\mathbf{O}$ core acts as the active species. This rotation is neccessary for the reaction to happen and was found to be the rate determining step. 101

\subsection{Binding and activation of sulfur by copper enzymes}

Over the past decades, examples of copper-sulfur interactions in biology sparked interest in research of copper thiolate chemistry. $102+104$ In nature, several copper-thiolate centers exist, eg. in blue copper proteins such as the mononuclear type- 1 and the dinuclear $\mathrm{Cu}_{\mathrm{A}}$ sites $\frac{105}{105}$ and copper chaperone proteins. ${ }^{106}$ Of special interest is a tetracopper-sulfide cluster $\left(\mathrm{Cu}_{\mathrm{Z}}\right.$-cluster $)$ found in nitrous oxide reductase $\left(\mathrm{N}_{2} \mathrm{OR}\right) . \underline{107}$

Driven by these natural role models, copper complexes that react with sulfur have been investigated and the products were found to exhibit similar binding motifs to those of $\mathrm{Cu} / \mathrm{O}_{2}$-intermediates. KARLIN found that a copper(I)-tris(2-pyridylmethyl)amine (TMPA) complex reacts with elemental sulfur $\left(\mathrm{S}_{8}\right)$ to form the end-on trans- $\mu-1,2-$ disulfide complex $[(\mathrm{TMPACu}-\mathrm{S}-\mathrm{S}-\mathrm{Cu}(\mathrm{TMPA})] .108$ KITAJIMA and coworkers were able to obtain a dicopper(II) $\mu-\eta^{2}: \eta^{2}$-disulfide complex from $\left[\mathrm{Cu}\left(\mathrm{SCPh}_{3}\left(\mathrm{HB}-3,5^{i} \mathrm{Pr}_{2} \mathrm{pz}\right)_{3}\right]\right.$. 104 Both complexes were found to be structural analogues of their copper-dioxygen counterparts and exhibit very similar spectroscopic features. ${ }^{109}$ Several more studies in the field of copper-sulfur interaction and copper-sulfide complexes have been carried out. $110-113$

\subsection{Homogenous copper catalyzed reactions}

Copper compounds have been proven useful in a broad variety of catalytic reactions. Besides the already mentioned click chemistry and GLASER- and ULMAN couplings, their main use is in oxygenation and oxidation reactions due to their interaction with dioxygen. Coupled phenols are present in a number of natural products and very useful in materials chemistry which has triggered research into their selective synthesis. 114 One-electron oxidation is favorable due to the phenolic stabilization of the radical species. These reactions can be carried out under mild conditions and tolerate many functional goups in contrast to other more sensitive biaryl coupling methods like KUMADA- or SUzUKIcoupling. 115,117 Additionally, functional groups can be introduced into previously nonfunctional positions, thus avoiding the need for prefuncationalized derivatives such as halides or boronoic acids. On the down side, regio-directed functionalization is more challenging but still accessible through the choice of catalyst. Still, meta-coupling is unnattainable owing to the electronic resonance structures of phenolic compounds. The 
mechanism of these reactions preceeds via deprotonation and subsequent electron transfer or direct hydrogen atom abstraction.

\subsubsection{Oxidative coupling of phenols}

Copper catalysts are highly effective in phenol coupling. When two out of the three possible positions for oxidative coupling are blocked, the reaction is regioselective. When the ortho-positions are blocked, regioselective para-coupling can be obtained under mild conditions. The same is observerd, if only one ortho-position is availabe (Scheme 1.7). When the structure of the resulting diphenol permits, overoxidation to the corresponding

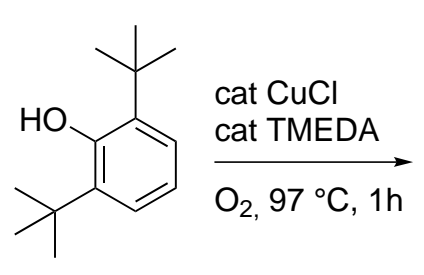<smiles>CC(C)(C)c1cc(-c2cc(C(C)(C)C)c(O)c(C(C)(C)C)c2)cc(C(C)(C)C)c1O</smiles><smiles>CC(C)(C)C1=CC(=C2C=C(C(C)(C)C)C(=O)C(C(C)(C)C)=C2)C=C(C(C)(C)C)C1=O</smiles><smiles>CC(C)(C)c1ccc(O)c(C(C)(C)C)c1</smiles>

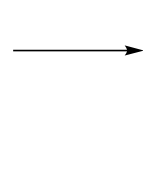<smiles>CC(C)(C)c1cc(-c2cc(C(C)(C)C)cc(C(C)(C)C)c2O)c(O)c(C(C)(C)C)c1</smiles>

Scheme 1.5 Regioselective copper catalyzed phenol coupling.

quinone is commonly observed.

The classic reaction involving the coupling of a phenol is the ULMANN-reaction, discovered in 1904, wherein a phenol is coupled to an aryl halide, using a copper(I)catalyst, yielding a diaryl ether. 12 12118

OTTENWAELDER, LUMB and coworkers carried out a lot of research to study the influences that govern the reactivity of copper catalyzed oxidation and oxygenation of phenolic substrates. $69 \mid 71811119$ [130 A variety of ligands, bases, additives and conditions were tested for the conversion of different phenols. They found that, indepedent of the employed ligand system, molecular sieves $(4 \AA)$ drastically increase the yield of the reaction. It was theorized that the removal of water from the reaction mixture is beneficial for the reaction. 
Employing the same di-tert-butyl ethylene diamine/copper(I) system that was used by STACK and coworkers to form reactive copper/oxygen intermediates, they were able to oxygenate phenolic substrates, substituted in 4-position, to ortho-quinones that undergo 1,4-addition with another equivalent of substrate to form 4-aryloxy-ortho-quinones. By choice of solvent and catalyst, these quinones could be transformed selectively either to 4alkoxy-ortho-quinones or the corresponding 2-alkoxy-para-quinone (Scheme 1.6. ${ }^{\text {[711811123] }}$<smiles>CC(C)(C(=O)NP)c1ccc(O)cc1</smiles>

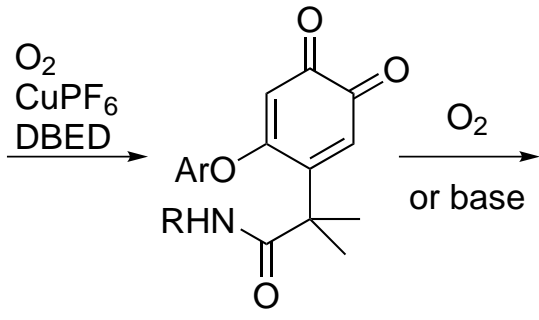<smiles>CC1(C)C(=O)NC2=CC(=O)C(=O)C=C21</smiles>

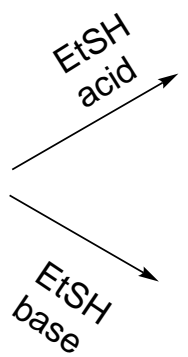<smiles>CC1(C)C(=O)Nc2cc(O)c(O)cc21</smiles>

Scheme 1.6 Synthesis of a 4-aryloxy-ortho-quinone and subsequent intramolecular ring closing reaction

Inspired by melanogenesis, they were able to perform intramolecular ring closing reactions based on their previous findings(Scheme 1.6). A generated 4-aryloxy-ortho-quinone undergoes substitution by an amine or amide function of the substrate to form oxindolquinones that can be transformed into oxindoles and further functionalized in 2- and 3position. $69[119 \mid 123$ With this catalsyt system it was also possible to generate ortho-amines from 2,4-di-tert-butylphenol by reaction with amines, 121130 to form ortho-azophenols from reaction of 3,5-substituted phenols with hydrazines or hydrazides 129 and to generate benzoxazoles, benzoxazinones, ortho-aminophenols, $N$-aryl pyrrolidine and $N$-aryl pyrrole from 3,5-substituted phenols. 127

\section{Polymerization of phenols}

In 1959 , it was discovered by $\mathrm{HAY}$ that $\mathrm{a} \mathrm{Cu}^{+}$pyridine complex catalyzes the oxidative para $\mathrm{C}-\mathrm{C}$ coupling of phenols and the subsequent oxidation of the 4-4'diphenols to the corresponding quinones, as well as $\mathrm{C}-\mathrm{O}$ coupling of 2,6 substituted phenols in the presence of dioxygen to a polyphenylene ether (PPE) (Scheme 1.7). 131 Further studies revealed that small ortho-substituents direct the reaction towards the $\mathrm{C}-\mathrm{O}$ coupled product and bulkier groups favour the formation of the $\mathrm{C}-\mathrm{C}$ coupled product. 132 
PPE is a versatile material for the engneering of thermoplastics. It makes for an excellent

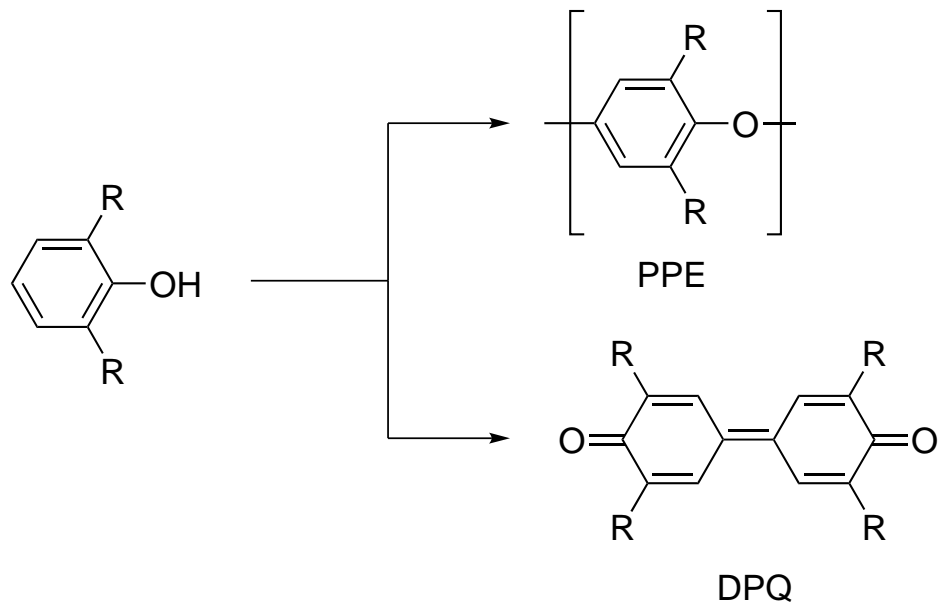

Scheme 1.7 Possible products of phenol coupling.

insulator as it absorbs unusually low amounts of moisture and maintains its properties over a wide humidity and temperature range. It also has a high glass transition temperature and is resistant to a variety of chemical compounds, water, salt solutions, acids and bases and is even a flame retardant. However, blending with polystyrene is neccessary to make processing into materials possible. Industrial synthesis is carried out in toluene with a copper(I) amine catalyst under 1-4 bars of oxygen pressure. These conditions make the recovery of the organic solvent as well as the use of anti-explosive reactors neccessary. Therefore, a lot of research is undertaken to open the possibility of a reaction in aqueous media. ${ }^{133 \mid 134}$ REEDIJK and corworkers optimized the reaction and were able to selectively obtain PPE with a molecular weight $M_{\mathrm{w}}$ of up to $86 \mathrm{kDa}$, employing a biphasic tolulene/water emulsion with a copper/imidazole catalyst. Still, the results highly depended on the ligand to metal ratio. ${ }^{135}$ Further influences governing the reation are the denticity and basicity of the ligand. It was also possible to carry out the reaction in aqueous sodium hydroxide solution at $50{ }^{\circ} \mathrm{C}$ under vigorous stirring with tmeda/ $\mathrm{CuCl}_{2}$ as the catalyst. However, under these conditions the $\mathrm{C}-\mathrm{C}$ coupled DPQ was also formed as a trace byproduct and the $M_{\mathrm{w}}$ was only $29 \mathrm{kDa}$. ${ }^{136}$

Several more attempts, employing surfactants like sodium- $n$-dodecyle sulfate, to create water based systems were undertaken with charged ligands ${ }^{134}$, naturally occuring amine ligands ${ }^{137}$, triazayclononane ligands ${ }^{138}$, polymeric, recyclable ligands ${ }^{139}$ and amine/carboxylate ligands. ${ }^{140}$ However, all of these systems either produce DPQ as a side product, give low molecular weight $\mathrm{PPE}$ or a high polydispersity. A recent attempt using Laccase, an enzyme that contains a tri-copper core and plays a role in lignin synthesis, gave low molecular weight polymers but with polydisperities down to 1.07. However, catecholes were used as substrates in these experiments and not DMP. 141

The mechanism of this polymerization is still under debate and two likely reaction mech- 
anisms have been proposed Scheme 1.8. The first one is an ionic pathway in which

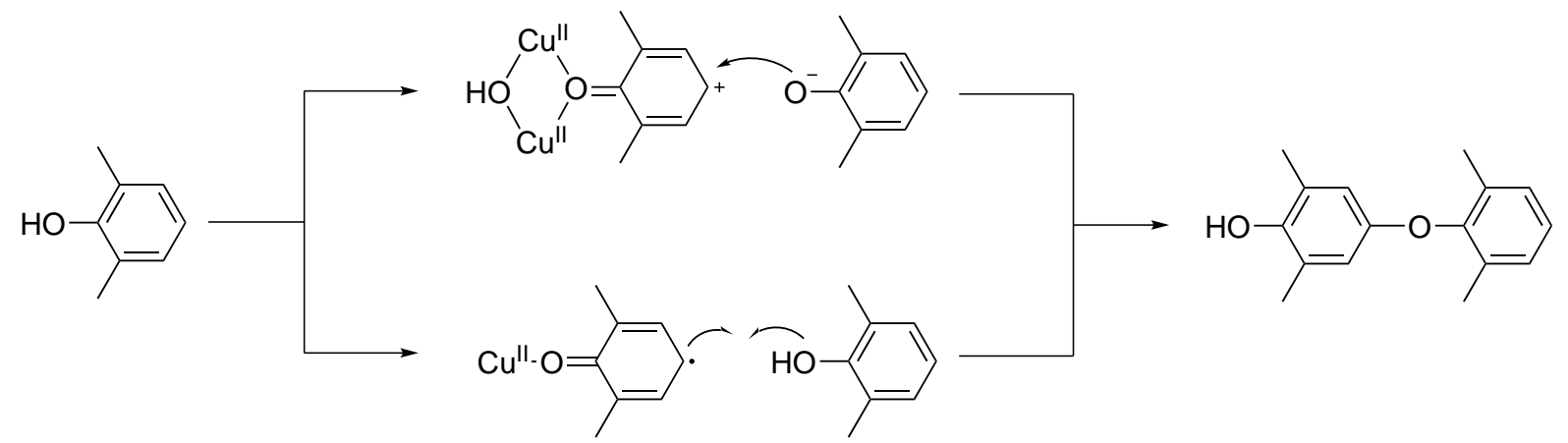

Scheme 1.8 Representation of the two most likely pathways of polymerization of 2,6dimethylphenol.

a phenolate reacts with a phenoxonium cation that is bound to two copper ions in a bridging manner Scheme 1.8). The second one is a radical mechanism wherein a phenoxy radical is generated by one electron oxidation through interaction of one phenol with one copper ion. A third mechanism was proposed that involves the coupling of freely dissolved phenoxy radicals but is rather unlikely as there is lack of evidence for the existnence of such a species. Under anaerobic conditions the presence of copper(II)-phenoxy complexes was observed, yet there is no EPR-evidence for the formation of a radical species, except when 2,4,6-tri-tert-butyl phenol was used as the substrate. Theoretical calculations of atomic charges in 2,6-dimethylphenol favour the ionic mechanism, with a phenoxonium ion proposed as the key intermediate. This species undergoes nucleophilic attack at the para-carbon by another phenolate to give the dimer. $6[142[143$

\subsection{2 $\mathrm{C}-\mathrm{H}$ bond activation}

While the activation of $\mathrm{sp}^{2}$ hybridized $\mathrm{C}-\mathrm{H}$ bonds has already developed into an impressive and versatile field and even was awarded with a nobel prize, $\stackrel{144}{ }$ the activation of $\mathrm{sp}^{3}$ hybridized bonds, however, still remains a challenge and has not found many applications. In general, $\mathrm{C}-\mathrm{H}$ bond activation is among the most challenging and versatile fields for synthetic chemists. Its utilisation enabled the laboratory synthesis of many natural products and functional molecules. Furthermore, it facilitates late stage functionalization and diversification and thus enables synthetic routes or products previously inaccessible to the synthetic chemist. 145] Additionally, the functionalization of alkanes grants access to a feedstock of starting materials and is connected to the accessibility of renewable energy.

Iтон and coworkers found that $\beta$-diketiminato ligands of the type $\mathbf{V}$ (Figure 1.13) form complexes with copper(I) and copper(II) salts. ${ }^{146 \mid 147]}$ By reaction of the copper(I) species with $\mathrm{O}_{2}$ or the copper(II) species with $\mathrm{H}_{2} \mathrm{O}_{2}$ a bis( $\mu$-oxo)dicopper(III) species 


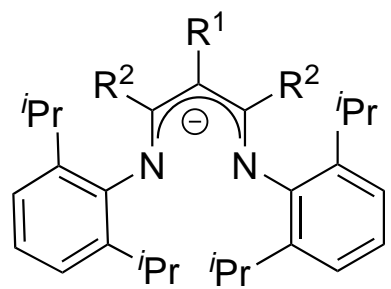

TMP1

Figure 1.13 $\beta$-diketiminato ligand used by ITOH and coworkers. $146 \mid 147$

was obtained. These species were able to oxygenate adamantane and cyclohexane and can be viewed as pMMO functional models. 59

MURAHASHI and coworkers reported the oxidation of cyclohexane to cyclohexanone and cyclohexanol in the presene of $\mathrm{CuCl}_{2}$, acetaldehyde, and 18-crown- 6 under $1 \mathrm{~atm}$ of oxygen at $70{ }^{\circ} \mathrm{C}$. They achieved a total yield of $61 \%$ cyclohexanone corresponding to a TON of 16000. 1481149

Several copper(I) complexes based on N-donor ligands have been reported for the oxidation of alkanes, alkenes, and alkohols using ${ }^{t} \mathrm{BuOOH}$ and one atmosphere of oxygen. Initially, these copper(I) complexes did not activate dioxygen but upon treatment with ${ }^{t} \mathrm{BuOOH}$ formed an intermediate adduct that subsequenty reacted with dioxygen to form a superoxide species. It was possible to oxidize ethylbenzene to acetophenone in $60 \%$ yield without the formation of side products. $\frac{150}{150}$

A frequently used model substrate for benzylic $\mathrm{C}-\mathrm{H}$ activation is the already mentioned 2,6-dimethyl phenol (DMP) as well as 2,4,6-trimethyl phenol (TMP), that cannot undergo $\mathrm{C}-\mathrm{O}$ polymerization. In the presence of $\mathrm{CuCl}_{2}$, an amine or oxime ligand and methanol, the copper systems selectively oxidize the para methyl group of TMP to the corresponding aldehyde, 3,5-dimethyl-4-hydroxy benzaldehyde (HDB). This transformation bears similarities to the oxygenation of benzylic $\mathrm{CH}_{3}$ groups as catalyzed by laccase and vanillyl alcohol oxidase. 151|152

REEDIJK and coworkers reported the stoichiometric oxidation of TMP to HDB with a $\left[\mathrm{CuCl}_{2}\right.$ (neo)]/NaOMe (neo $=2,9$-dimethylphenanthroline) system. The reaction could be run catalytically if hydrogen peroxide was used in refluxing $\mathrm{MeOH}$. 153.

Upon decreasing the amount of NaOMe, a monosubstituted intermediate could be isolated. It has been proposed that the reaction proceeds via a two electron oxidized quinone methide intermediate (VI) that undergoes repeated 1,6-addition of an alcohol to first form an ether (MDP) that is further substituted to form an acetal (VII) that is hydrolyzed and gives the aldehyde (HDB) Scheme 1.9. 154 By addition of the unoxidizable pentafluorophenol, it was possible to get a snapshot of the initial binding step of the phenol (Figure 1.14). Reactions were carried out in DMF with NaOMe to 


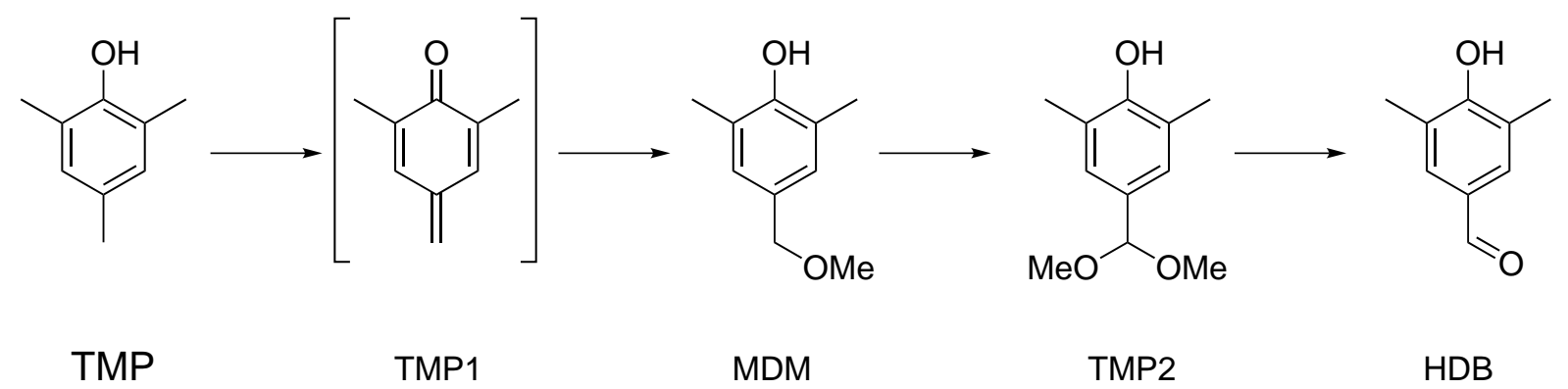

Scheme 1.9 Stepwise 1,6-addition of MeOH to TMP to yield HDB.

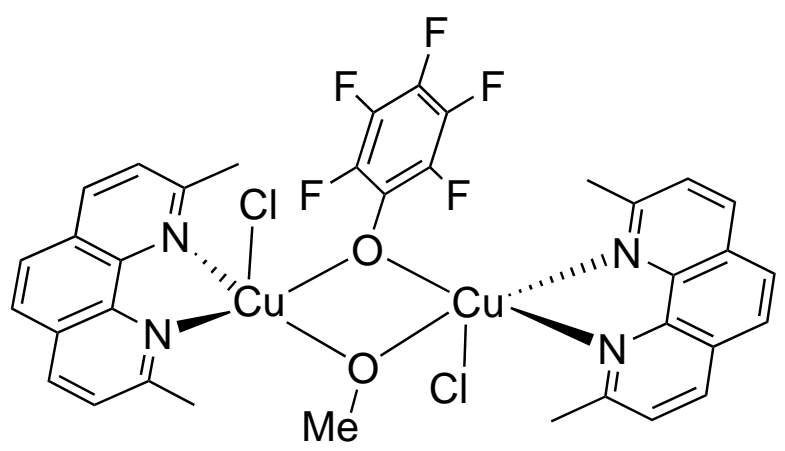

Figure 1.14 Representation of pentafluorophenol bound to the $[\mathrm{CuCl}(\mathrm{neo})]_{2}$ complex.

deprotonate the para- $\mathrm{CH}_{3}$ group to form the proposed quinone methide intermediate. In the absence of the neo-ligand no reaction occured, thus emphaszing the need for N-donor ligands to stabilize the intermediate copper(I) state.

\subsection{Current state of research}

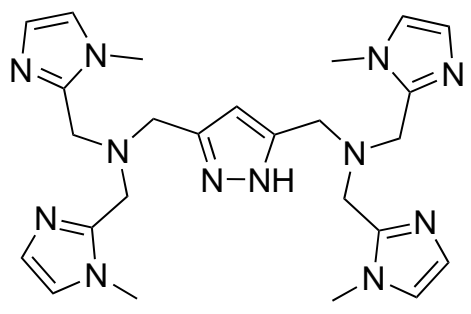

$\mathrm{HL}^{1}$

Figure 1.15 Pyrazolate based binucleating ligand $\mathrm{HL}^{1}$ synthesized by PROKOFIEVA.

Preceeding this work, A. PROKOFIEva synthesized the pyrazole based binucleating ligand $\mathrm{HL}^{1}$ (Figure 1.15) and its corresponding dicopper(II) complex $\left[\mathbf{L}^{1} \mathrm{Cu}_{2}(\mathrm{MeOH})(\mathrm{OMe})\right]\left(\mathrm{ClO}_{4}\right)_{2}(\mathbf{1})$ Figure 1.16) and investigated $\mathbf{1}$ towards the catalytic aerobic oxidation of 2,4,6-trimethyl phenol (TMP). 155 It was found that $\mathbf{1}$ 


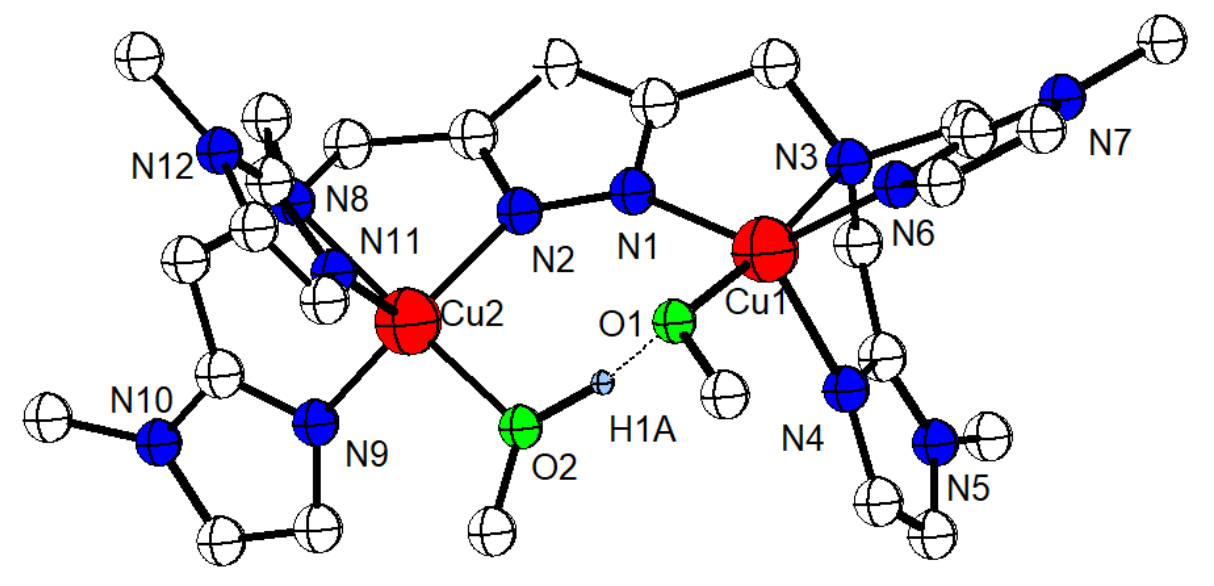

Figure 1.16 Molecular structure of bioinspired dinuclear compartmental dicopper(II) complex $\left[\mathbf{L}^{1} \mathrm{Cu}_{2}(\mathrm{MeOH})(\mathrm{OMe})\right]\left(\mathrm{ClO}_{4}\right)_{2}(\mathbf{1})$ synthesized and characterized by A. Prokofieva. 155.

was able to oxidatively $\mathrm{C}-\mathrm{C}$ couple two molecules of 2,6-dimethyl phenol (DMP) to give 3,3',5,5'-tetramethyl-4,4'-dihydoxy-biphenyl and its subsequent oxidation product 3,3',5,5'-tetramethyl-4,4'-diphenoquinone (DPQ). Related reactivity was observed for 2,4,6-trimethyl phenol (TMP), which has been coupled at its para- $\mathrm{CH}_{3}$ group to form 4,4'-dihydroxy-3,3',5,5'tetramethyl bibenzyl (TMBB) (Scheme 1.10). TMBB is subse-<smiles>CC1=CC(=CC=C2C=C(C)C(=O)C(C)=C2)C=C(C)C1=O</smiles><smiles>Cc1cc(CCc2cc(C)c(O)c(C)c2)cc(C)c1O</smiles>

Scheme $1.10 \mathrm{C}-\mathrm{C}$ coupling reaction of TMP catalyzed by $\mathbf{1}$

quently oxidized to 3,3 ',5,5'-tetramethylstilbene-4,4'-quinone (TMSQ) by dicopper complex 1. Under an inert atmosphere the coupling of two molecules of TMP occured via one electron oxidation of a copper-bound phenolate, forming a mixed valent $\mathrm{Cu}^{\mathrm{I}} \mathrm{Cu}^{\mathrm{II}}$ species and half a molecule of TMBB. The formation of one molecule TMBB is a $2 e^{-} / 2 \mathrm{H}^{+}$ process. In the presence of dioxygen TMBB was further oxidized to TMSQ in a $4 e^{-} / 4$ $\mathrm{H}^{+}$process Scheme 1.10. The dicopper complex 1 in MeCN (Figure 1.17) shows an absorption feature at $989 \mathrm{~nm}$, typical for a $d$ - $d$ transition. Upon addition of TMP, the 


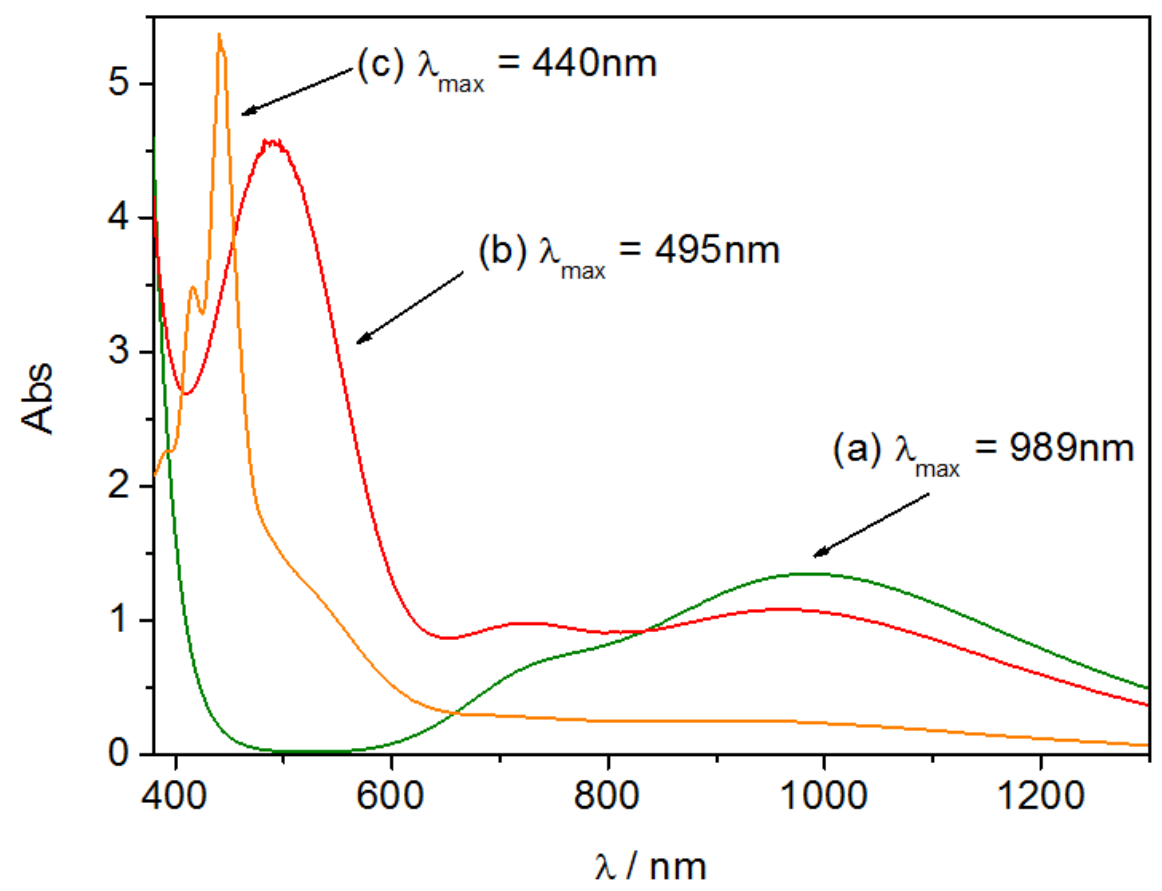

Figure $\mathbf{1 . 1 7} \mathrm{UV} / \mathrm{Vis}$ absorption spectra at room temperature of a) $\mathbf{1}$ (MeCN, $c=$ $\left.5 \cdot 10^{-3} \mathrm{M}\right) \mathbf{b}$ ) excess TMP and $\mathbf{1}$ in $\left.\left(\mathrm{MeCN}, c=5 \cdot 10^{-3} \mathrm{M}\right) \mathbf{c}\right)$ TMSQ in (MeCN, $c=5 \cdot 10^{-5} \mathrm{M} \cdot 155$

previously feautureless region around $500 \mathrm{~nm}$ shows an intense band at $495 \mathrm{~nm}(\epsilon \sim 1500$ $\left.\mathrm{L} \cdot \mathrm{mol}^{-1} \cdot \mathrm{cm}^{-1}\right)$, resulting in a deep red colored solution.

Thise band was identified as a copper(II) $\leftarrow$ phenolate ligand-to-metal-charge-transfer transition on the basis of UV/Vis and resonance Raman spectroscopy. The binding stoichiometry of TMP and $\mathbf{1}$ was determined to be 1:1 using the Job plot method. The phenolic substrate is deprotonated by the endegenous methoxide and subsequently binds as a phenoxide to the copper(II) ion. The binding and reactivity of other phenols largely depends on their electronic properties and their steric demand. Substituents in ortho-position block the correspondig ortho-coupling. Substitution in ortho and meta-position can completely inhibit any kind of reactivity.

When substrates are used that cannot be oxidized, the complex remains in its dicopper(II) state with the phenol bound to one of the metal ions (Figure 1.18). 156

The final organic products have been identified by column separation and NMR techniques. EPR was used to determine the oxidation state of the dicopper complex after the reaction under inert atmosphere. An EPR spectrum of $\mathbf{1}$ in MeCN recorded at $50 \mathrm{~K}$ is depicted in Figure 1.19. 


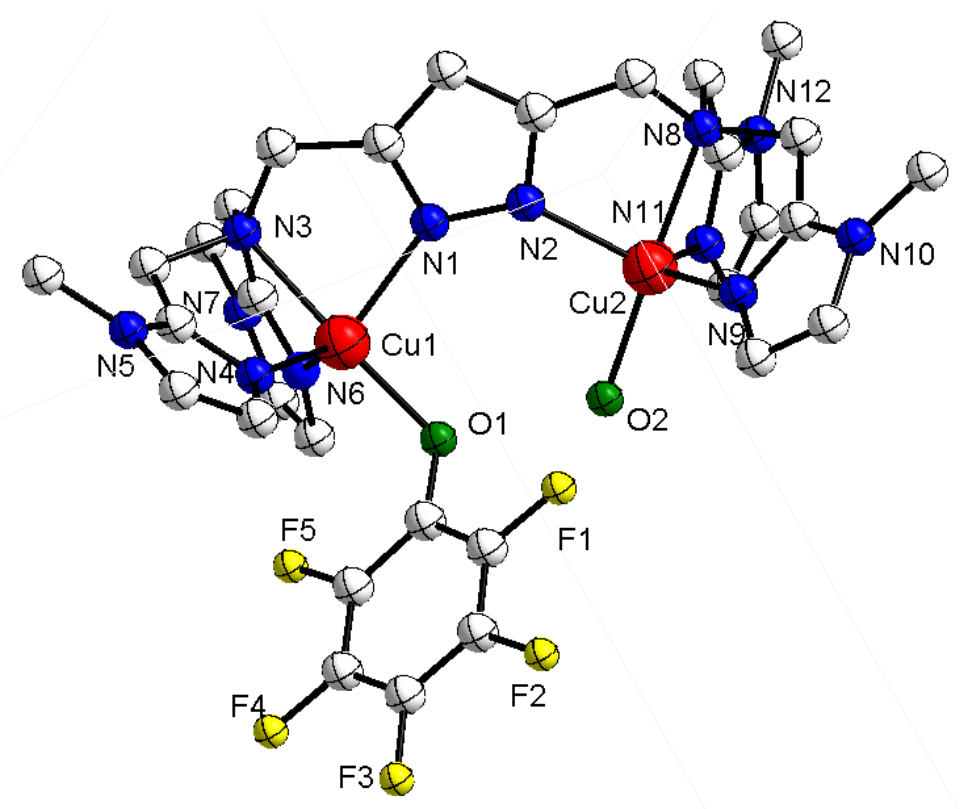

Figure 1.18 Molecular structure of Pentafluorophenolate bound to the dicopper(II) complex. 156
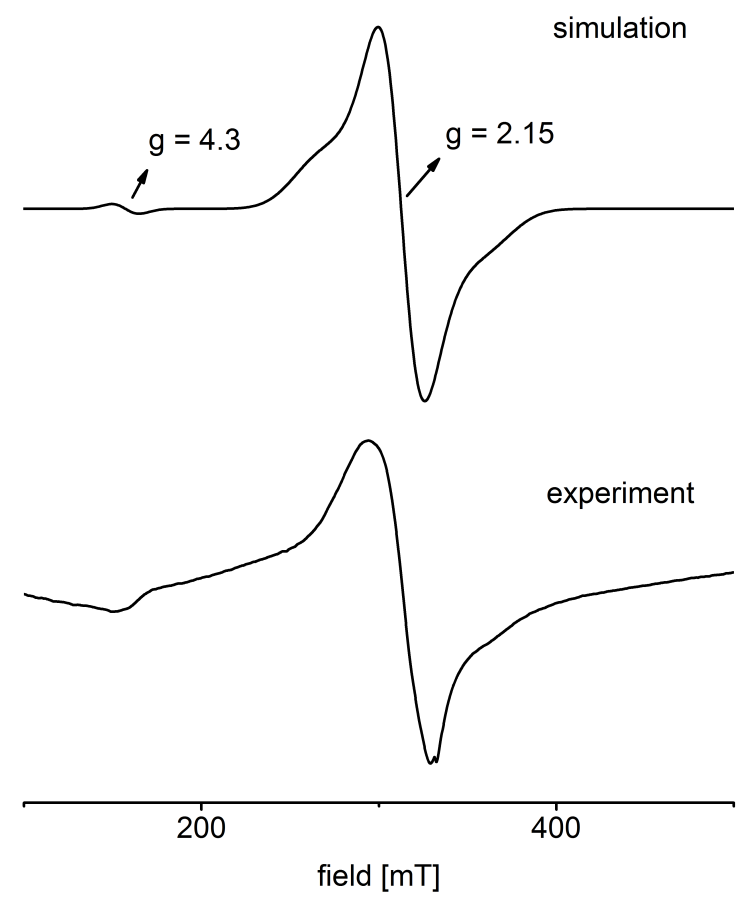

Figure 1.19 EPR spectrum of 1 in MeCN recorded at $150 \mathrm{~K}^{156}$ and simulated pattern. The $\mathrm{g}_{\|}$and $\mathrm{g}_{\perp}$ both show a value of 2.15 and an $\mathrm{A}$ value of $0.0320 \mathrm{~cm}^{-1}$ and a half field signal at $\mathrm{g}=4.3$ (additional parameters: nucleus $=\mathrm{Cu}, \mathrm{Cu}$, total $\mathrm{spin}=1$, frequency $=$ 9.427 GHz, $\mathrm{T}=150 \mathrm{~K}$, linewidth $=8 \mathrm{mT}$, gstrain $=[0.2,0.1,0.1])$.

A catalytic cycle was proposed, based on the data obtained (Scheme 1.11). While good insight into the mechanism was provided, several aspects remained unknown. Further 


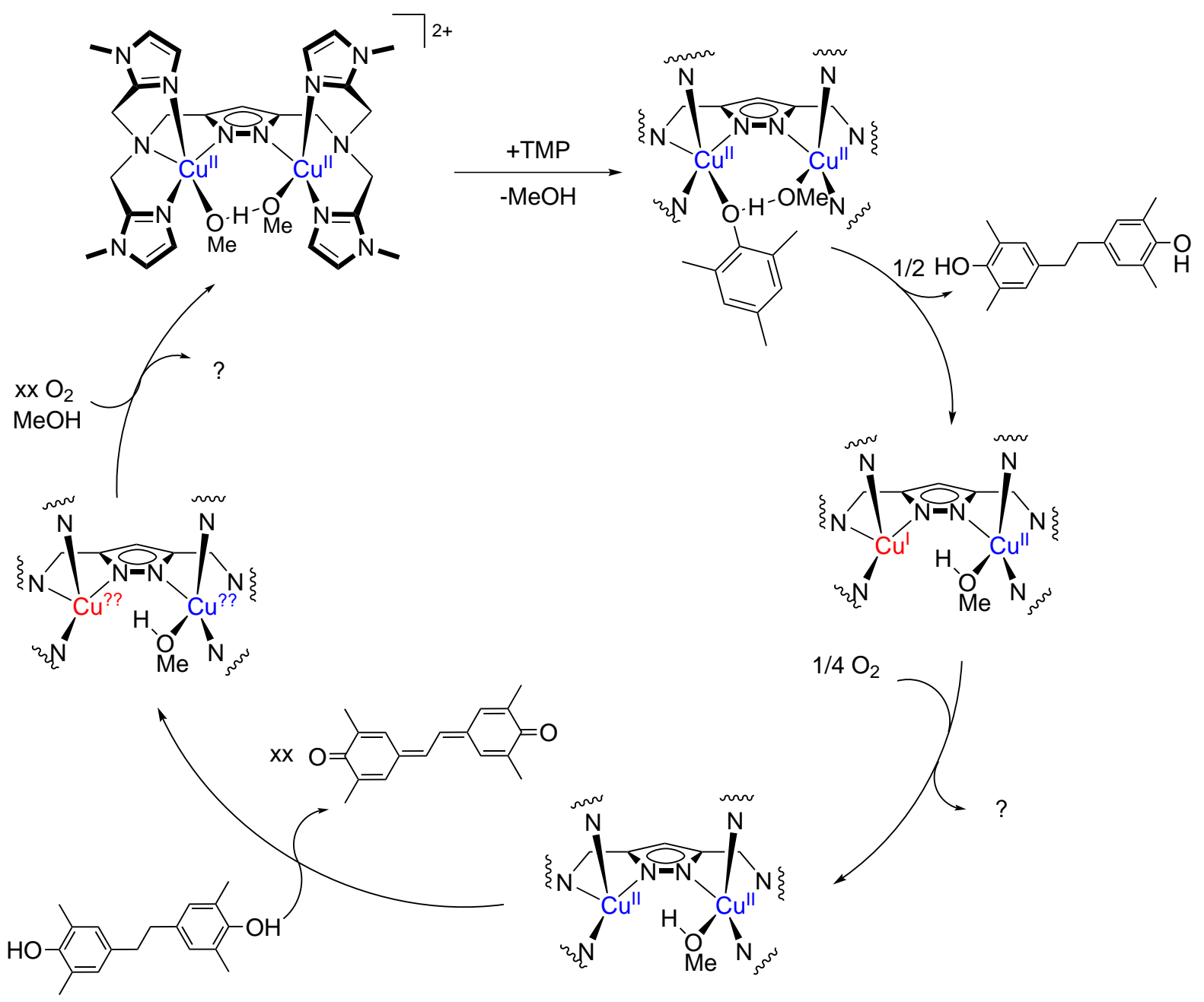

Scheme 1.11 Proposed catalytic cycle by Prokofieva.

investigations to determine the oxidation state of the dicopper complex after the reaction with TMBB are neccessary. Furthermore, the fate of the $\mathrm{O}_{2}$ molecule involved in the reoxidation of the copper(I)/copper(II) species has not been determined.

\subsubsection{Spectroscopy of copper-phenolate adducts}

One aspect of the investigation is the nature of the adduct of the copper bound phenolate. In this work a copper(II) complex is reacted with a phenolate to form an adduct. This

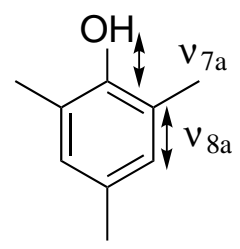

Figure 1.20 2,4,6-trimethylphenol and representation of relevant stretching frequencies for resonance Raman investigations. 
adduct can either remain a copper(II)-phenoxide species until a reaction occurs or undergo electron transfer from the phenolate to the copper. Hereby the copper(II) is reduced to copper(I) and a phenoxyl radical is formed. IR and Resonance Raman spectroscopy provides a powerful tool for determining the adduct that is present in solution. There are three stretching frequencies that are indicative for the respective species. The $\mathrm{Cu}_{-}^{-}$ $\mathrm{O}$ stretch, the $\mathrm{C}-\mathrm{O}$ stretch $\left(\nu_{7 \mathrm{a}}\right)$ and the $\mathrm{C}_{\text {ortho }}-\mathrm{C}_{\text {meta }}$ stretch $\left(\nu_{8 \mathrm{a}}\right)$. The $\mathrm{Cu}-\mathrm{O}$ stretch was too weak in intensity and often overlaped with other peaks and was therefore not considered in this work The $\nu_{7 a}$ mode ranges from $1500 \mathrm{~cm}^{-1}$ for phenoxyl radicals with the radical delocalized in the aromatic ring to below $1300 \mathrm{~cm}^{-1}$ for $\mathrm{C}-\mathrm{O}$ single bonds in phenols. The $\nu_{8 \mathrm{a}}$ is found at around $1600 \mathrm{~cm}^{-1}$ and exhibits an increasing blue shift with increasing radical character. $157-165$ 



\section{Objective}

Industrially applied oxidation reactions very often require harsh reaction conditions and a stoichiometric amount of oxidant, which are often highly toxic like permanganates or chromates. In contrast to this, natural copper enzymes are able to oxidize substrates using only readily available and abundant aerial dioxygen as the oxidant. Furthermore, these enzymes can mediate reactions under ambient conditions and are often extremely efficient, and are therefore an inspiration for the fields of bioinorganic chemistry and sustainable catalysis. Aside from catalysis, model chemistry can provide valuable insight into mechanisms and can identify reactive intermediates in biological systems.

Bioinspired dicopper complexes based on pyrazolate ligand scaffolds with amine side arms were recently shown to exhibit suitable reactivity for the desired transformation of phenolic substrates. These systems can be easily modified with different chelating side arms in 3- and 5-positions of the heterocyclic core.

The main factor influencing structure and reactivity of bioinspired complexes is the

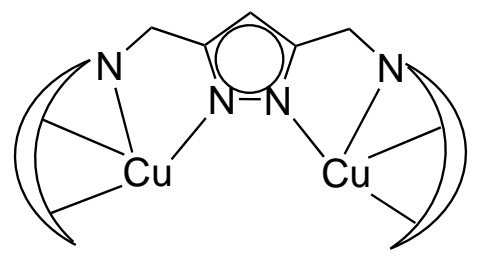

Figure 2.1 pyrazolate based ligand scaffold

coordination sphere, provided by the chelating side arms, and the resulting metal-metal sepearation. By varying the side arms, the influence of the aforementioned factors will be investigated.

The first objective of this work was to further elucidate the oxidation mechanism of TMP by $\mathbf{1}$ and fill in the gaps of the proposed catalytic cycle (Scheme 1.11). Furthermore, expansion of the scope of reactivity of $\mathbf{1}$ is sought.

The second goal is to synthesze new ligands by variation of the side arms. To achieve this, new amine side arms will be synthesized applying known and established as well as new synthetic strategies. These ligands will be used to generate new dicopper(I) and dicopper(II) complexes and characterize them. These complexes will be investigated towards their catalytic activity with respect to oxidation reactions involving molecular dioxygen. Complexes shown to be catalytically active are subject to mechanistic investigations. 



\section{Synthesis of pyrazolate based ligand scaffold}

\subsection{Pyrazolate based Ligand Frameworks}

Pyrazolate based dinucleating ligand frameworks bring together two tripodal tetradentate ligand compartments which have proven to be succesful in emulating the properties and reactivities of mononuclear N-ligated metalloenzymes. 166-170] Pyrazole finds wide application in bioinorganic model chemistry as a briding moiety for bringing together two metal ions in close proximity, besides the already mentioned alkoxide and phenoxide bridges. Furthermore, the monoanionic pyrazolate emulates other bridging moieties like carboxylate which are found in natural systems, while still leaving an open coordination site at the metal. The pyrazolate fuses two mononuclear coordination environments and enables tunable coordination environments and metal metal distances (from 3.40 to $4.50)[168[171-175$ which has been proven useful for enabling reactions that require metal metal cooperativity such as the activation of small molecules, as well as the binding and transformation of organic substrates.

The synthetic protocols developed to introduce a wide variety of side arms are based on the substitution in 3 - and 5-positions of the pyrazole to give symmetric compartmental ligands. Thanks to mostly facile coupling reactions, the variety of side arms ranges from (poly)pyridyl units to more elaborate systems involving various amines, imidazoles, carboxylate or even phosphorus donor-atoms. In this work, bioinspired amine sidearms containing different (benz)imidazole derivatives were employed. Imidazole ligation was chosen, due to it being the ligand in type-3 copper enzymes that gave the inspiration for this work. Coupling of the imidazole side arms to the pyrazole ring was achieved by nucleophilic substitution of chloro substituted pyrazole precursors.

These imidazole based ligands are bioinspired in nature and their dicopper complexes exhibit promising catalytic activity under mild conditions. Their chelating nature prevents partial dissociation of ligands as witnessed in open chain systems and provides high stability over a wide range of environments and can withstand even harsh reaction conditions such as high basicity and acidity as well as elevated temperatures. These advantages make these systems feasable for catalysis. $\mathbf{H L}^{1}$ has been reported previously ${ }^{[156}$ and builds the basis for expanding the scope of imidazole containing side arms. $\mathbf{H L}^{2}$ is a new ligand based on the work of CASELLA, $[86176$ with the side arms transferred from a xylene to a pyrazolate-bridge. $\mathbf{H L}^{3}$ has not been reported before. 


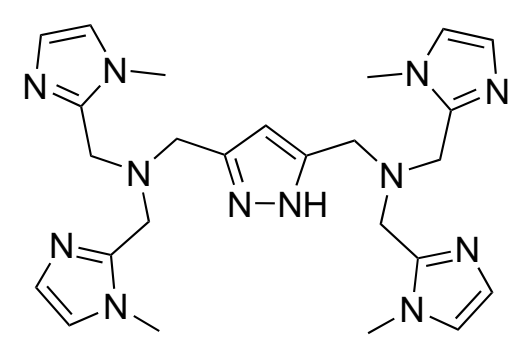

$\mathrm{HL}^{1}$

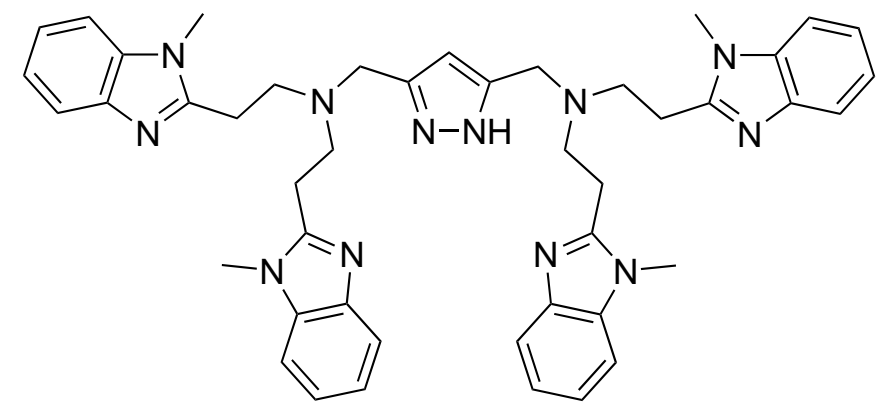

$\mathrm{HL}^{2}$

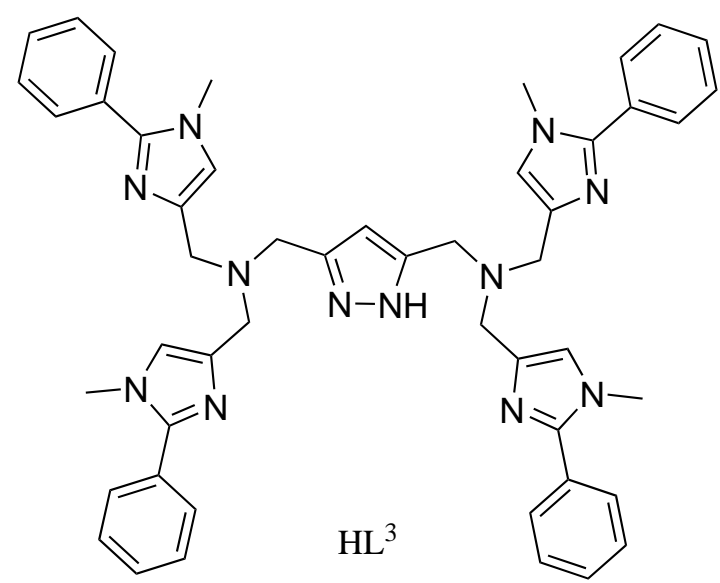

Figure 3.1 Pyrazolate based ligands $\mathbf{H L}^{1}-\mathbf{H L}^{3}$ used in this work. 


\subsection{Synthesis of building blocks}

\subsubsection{Synthesis of the pyrazole backbone}

The first step in the synthesis of all herein described ligands starts with the pyrazole backbones 3,5-bis(chloromethyl)pyrazole hydrochloride (e) and 3,5-Bis(chloromethyl)-1(tetrahydropyran-2-yl)-pyrazole (f). Both compounds haven been well established in the synthesis of various ligand systems and were synthesized according to literature $166|177| 178$ as depicted in Scheme 3.1. Protection of the NH unit by means of dihydropyrane (DHP) is neccessary in some cases to prevent oligomerization through nucleophilic attack of another pyrazole unit. Furthermore, NH-protection enables column chromatographic workup of the protected ligand. The synthesis of $\mathbf{f}$ starts from commercially available
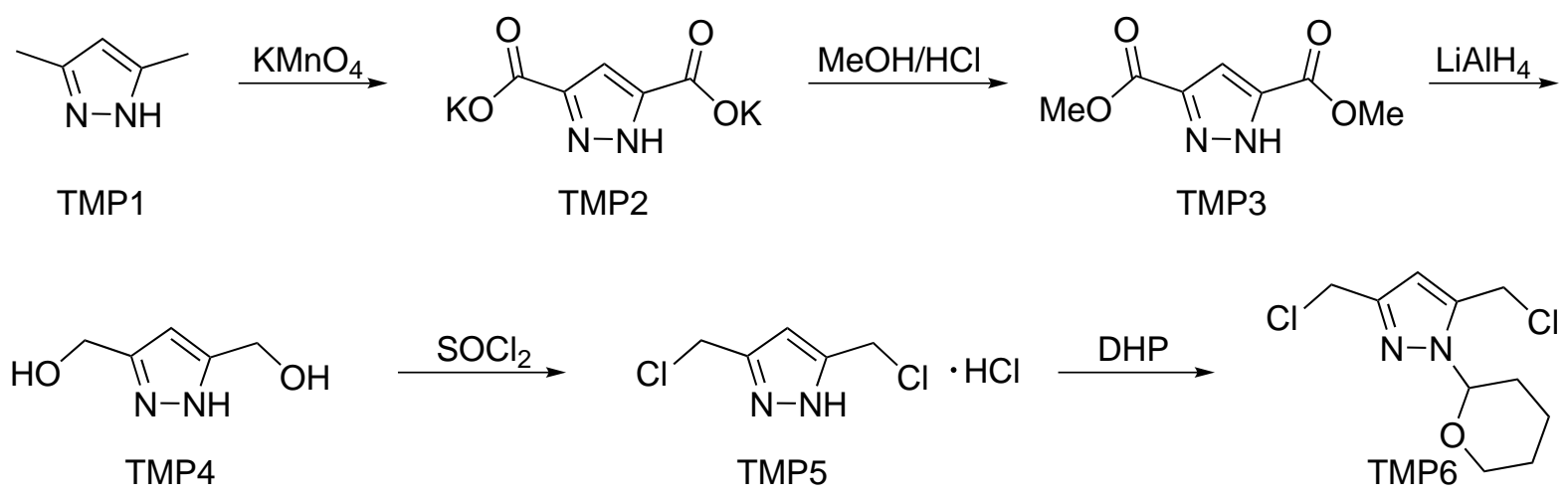

Scheme 3.1 Synthetic route to obtain the pyrazole building blocks $\mathbf{e}$ and $\mathbf{f}$.

3,5-dimethylprazole (a) which is transformed in a five-step synthesis (a-e). While the synthesis of $\mathbf{H L}^{1}$ can be achieved using the unprotetcted pyrazole derivative $\mathbf{e}$, which is obtained by treating $\mathbf{d}$ with thionylchloride, the synthesis of $\mathbf{H L}^{2}$ and $\mathbf{H} \mathbf{L}^{3}$ required the $\mathrm{NH}$ to be protected in order to perform chromatographic workup.

\subsubsection{Synthesis of sidearms}

Bis[(1-methylimidazol-2-yl)-methyl]amine $(\mathbf{j})$ was prepared in a four-step synthesis starting from $N$-methylimidazole $(\mathbf{g}) .{ }^{[179 \mid 180]}$ Deprotonation in 2-position by $n$-BuLi and subsequent treatment with DMF gave $N$-methyl-2-formyl-imidazole $(\mathbf{h})$. Further treatment with hydroxlamine hydrochloride afforded $N$-methylimidazolyl-2-oxime (i). Coupling of $\mathbf{i}$ and $\mathbf{h}$ and subsequent hydrogenation yielded the desired sidearm $\mathbf{j}$.

Introducing a longer sidechain to allow for more flexibility and bringing the metal ions closer together was achieved by synthesizing [(1-methylbenzimidazole-2-yl)ethyl]amine $(\mathbf{m})$. In a simple one-pot reaction in aqueous $\mathrm{HCl}$ solutio,n 3,3'-iminodipropiontrile (k) and $N$-methyl-1,2-phenylene diamine (l) are fused to give the desired product $\mathbf{m} .{ }^{86}$ 


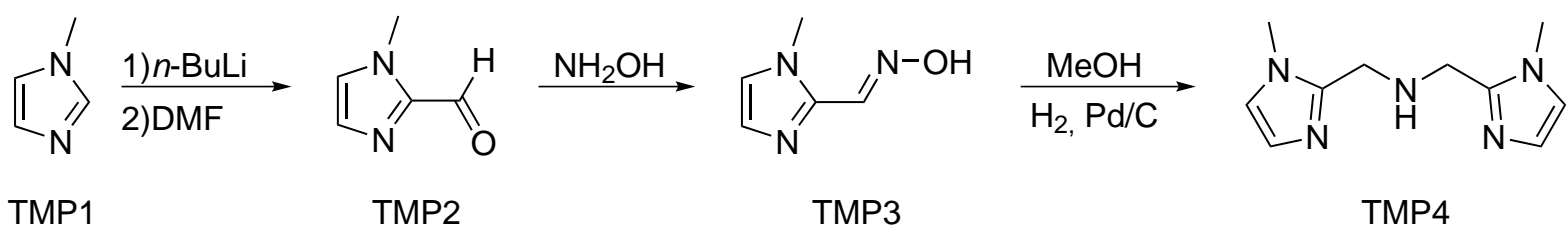

Scheme 3.2 Synthesis of bis[(1-methylimidazle-2-yl)methyl]amine $(\mathbf{j})$.<smiles>N#CCCNCCC#N</smiles>

TMP1<smiles>CNc1ccccc1N</smiles>

TMP2<smiles>Cn1c(CCNCCc2nc3ccccc3n2C)nc2ccccc21</smiles>

TMP3

Scheme 3.3 Synthesis of bis[(1-methylbenzimidazle-2-yl)ethyl]amine (m)

A more complex approach was neccessary in order to obtain the more shielding bis(1methyl-2-phenyl-4-imidazolylmethyl)amine (t) in a five-step synthesis ${ }^{181182}$ Coupling of<smiles>N=C(N)c1ccccc1</smiles>

TMP1<smiles>OCC1(O)COC(O)(CO)CO1</smiles>

TMP2<smiles>NC=CO</smiles><smiles>OCc1c[nH]c(-c2ccccc2)n1</smiles>

TMP3

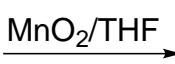<smiles>O=Cc1c[nH]c(-c2ccccc2)n1</smiles>

TMP4<smiles>CC(C)CC(=O)c1cn(C)c(-c2ccccc2)n1</smiles><smiles>CCN(Cc1ccccc1)Cc1cn(C)c(-c2ccccc2)n1</smiles>

TMP6<smiles>Cn1cc(CNCc2cn(C)c(-c3ccccc3)n2)nc1-c1ccccc1</smiles>

TMP7

Scheme 3.4 Synthesis of Bis(1-methyl-2-phenyl-4-imidazolylmethyl)amine t.

commercially available benzamidine hydrochloride (n), diacetone dihydroxide (o) and ammnonium chloride in concentrated ammonia solution afforded 4-hyroxymethyl-2-phenyl imidazole (p). Subsequently, the hydroxy group was oxidized to a formyl function (q) and the NH-function methylated $(\mathbf{r})$. The obtained compound was reductively coupled to benzyl amine to give benzyl-bis(1-methyl-2-phenyl-4-imidazolylmethyl)amine (s). Finally the benzyl group was removed by means of hydrogenation and the desired compound $(\mathbf{t})$ was obtained. 


\subsection{Synthesis of Ligands}

For the synthesis of the final ligands, very similar conditions were used in all cases, as depicted in Scheme 3.5. Dry MeCN or THF was used as solvent and sodium carbonate added as base.

For $\mathbf{H L}^{1}$ the unprotected pyrazole $\mathbf{e}$ was used and the coupling reaction executed in MeCN at elevated temperatures. For the synthesis of $\mathbf{H L}^{2}$ and $\mathbf{H} \mathbf{L}^{3}$ the protected pyrazole $\mathbf{f}$ was used and the reaction carried out in THF for the former ligand and in MeCN for the latter. In both cases tetrabutylammonium bromide was added in catalytic amounts. While $\mathbf{H L}^{1}$ could be used directly after workup of the reaction mixture, $\mathbf{H L}^{2}$ and $\mathbf{H L}^{3}$

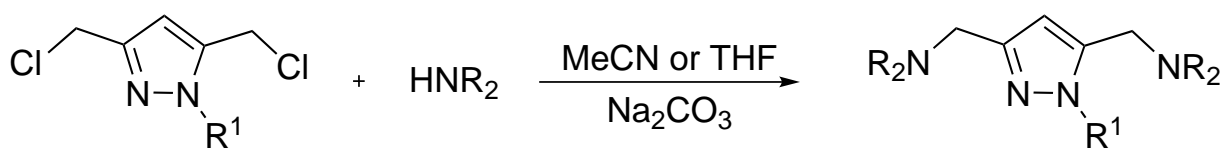

Scheme 3.5 Synthetic scheme for coupling the side arms to the pyrazole backbone.

required column chromatographic workup and subsequent deprotection using ethanolic $\mathrm{HCl}$ solution, in order to be obtained in sufficient purity. 



\section{Bioinspired oxidation reactions of phenols catalyzed by $\left[\mathrm{L}^{1} \mathrm{Cu}_{2}(\mathrm{MeOH})(\mathrm{OMe})\right]\left(\mathrm{ClO}_{4}\right)_{2}$}

In this chapter, bioinspired oxidation reactions of phenols catalyzed by a pyrazolate based compartmental dinuclear copper(II) complex of $\mathbf{H L}^{1}$ will be discussed in detail.In order to expand the investigations of PROKOFIEVA mentioned earlier, the mechanism of action of this dinuclear copper site will be further investigated, especially considering the involvement of dioxygen and the fate of the $\mathrm{O}_{2}$ molecule involved in reoxidation of the complex. Substrate binding motifs and substrate binding constants were investigated by means of $\mathrm{UV} /$ Vis absorption, resonance Raman and EPR spectroscopy and structure determination via X-ray crystallography. In selectively choosing specific solvents and reaction conditions a variety of phenolic products could be isolated that can be used as building blocks in organic synthesis.

\subsection{Mechanistic studies of the oxidation of TMBB}

To investigate the properties of the reaction of $\mathbf{1}$ and TMBB and compare it to TMP, it first had to be synthesized. The synthesis was achieved by dissolving TMP in $\mathrm{Et}_{2} \mathrm{O}$ and adding 0.6 equivalents of $\operatorname{Ag}_{2} \mathrm{O}$, which yielded a mixture of compounds that had to be seperated by using HPLC technique. Due to very similar retention times of TMBB and TMSQ the product could only be isolated in small quantities and was sometimes contained minor trace impurities of TMSQ. TMSQ is intensly coloured $\left(\epsilon \sim 96000 \mathrm{~L} \cdot \mathrm{cm}^{-1} \cdot \mathrm{mol}^{-1}\right)$ and interferred with $\mathrm{UV} / \mathrm{Vis}$ measurements. Any attempt to reduce TMSQ to TMBB was unsuccessful.

$U V / V i s$

When TMBB is added to a solution of $\mathbf{1}$ in MeCN, the rise of a LMCT band is observed Figure 4.1). Its absorption maximum is shifted by about $15 \mathrm{~nm}$ to $480 \mathrm{~nm}$ compared to the LMCT band that occured when TMP is added to a solution of $\mathbf{1}$.

resonance Raman

In order to gain more insight into the nature of these adducts, resonance Raman (rR) experiments were performed. Samples were prepared in $5 \mathrm{~mm}$ NMR tubes and frozen in liquid nitrogen. Excitation was done at $457 \mathrm{~nm}$ which overlaps well with the LMCT bands of the investigated adducts at $\lambda_{\max }=480 \mathrm{~nm}$ for $\mathbf{1} / \mathrm{TMBB}$ and $\lambda_{\max }=495 \mathrm{~nm}$ for $\mathbf{1} / \mathrm{TMP}$ and provides a resonance enhancement of the phenolate vibrations.

Regarding the nature of the adduct, the $\mathrm{C}-\mathrm{O}$ stretching frequency $\left(\nu_{7 \mathrm{a}}\right)$ and the 


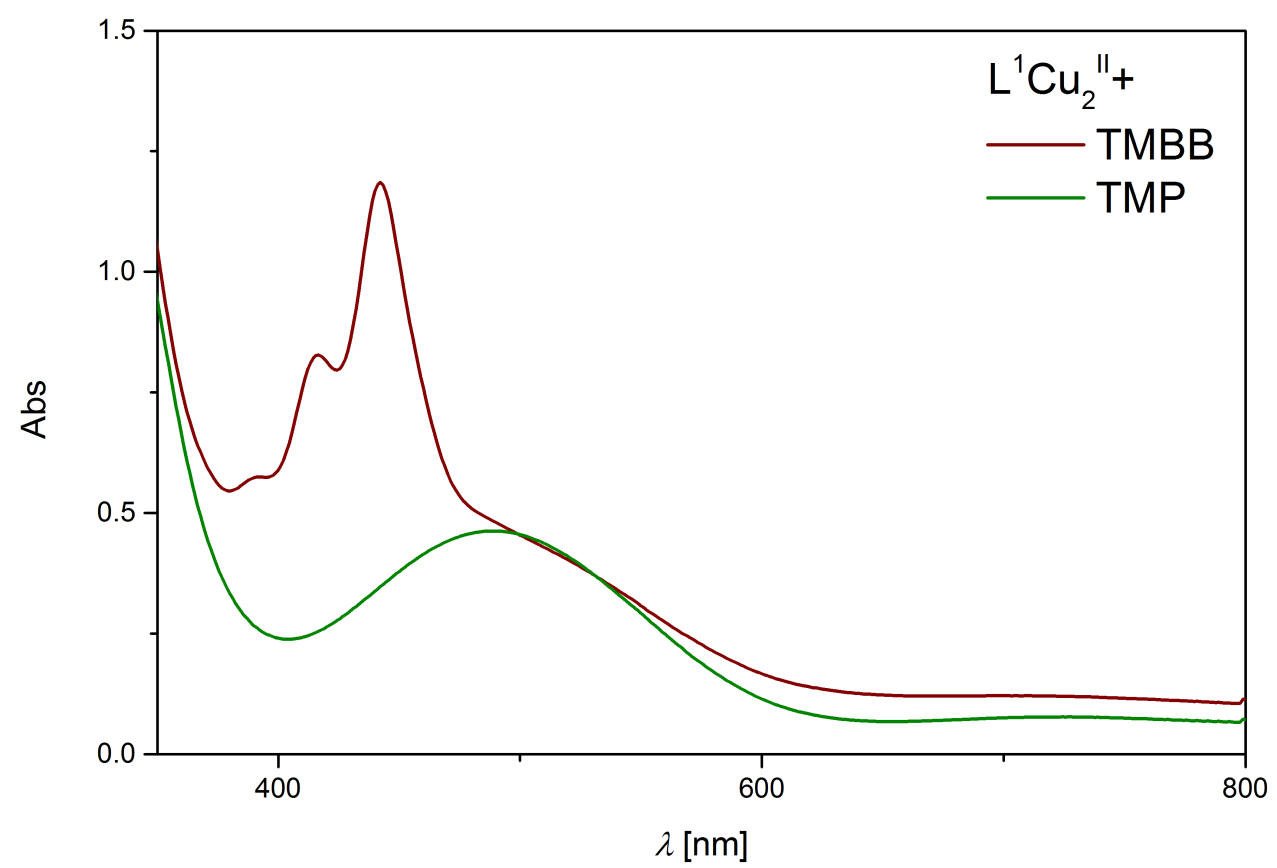

Figure 4.1 Comparison of absorption maximum of 1 (MeCN, $\left.c=5 \cdot 10^{-3} \mathrm{M}\right)$ and 15 equivalents of phenol. The absorptions maximum at $440 \mathrm{~nm}$ stems from TMSQ.

$\mathrm{C}_{\text {ortho }}-\mathrm{C}_{\text {meta }}$ stretching frequency $\left(\nu_{8 \mathrm{a}}\right)$ Figure 1.20 contain crucial information about the donating strength of the phenolate and its internal electronic structure. 157164165$]$ The $\nu_{7 a}$ mode ranges from $1500 \mathrm{~cm}^{-1}$ for phenoxyl radicals with the radical delocalized in the aromatic ring to below $1300 \mathrm{~cm}^{-1}$ for $\mathrm{C}-\mathrm{O}$ single bonds in phenols. The $\nu_{8 \mathrm{a}}$ is found at around $1600 \mathrm{~cm}^{-1}$ and exhibits an increasing blue shift with increasing radical character. This effect is due to a decrease in the corresponding $\mathrm{C}-\mathrm{O}$ and $\mathrm{C}-\mathrm{C}$ bond length that

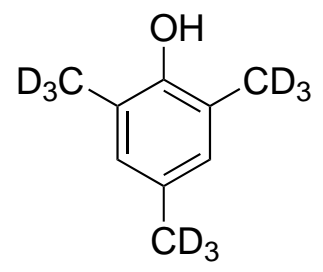

Figure 4.2 Structure of the $\mathrm{CD}_{3}$ substituted TMP-d ${ }^{9}$.

originates from the semiquinoid character of the radical. PROKOFIEVA also recorded a spectrum of the adduct of $\mathbf{1}$ and TMP- $\mathrm{d}^{9}$. This molecule Figure 4.2 bears $\mathrm{CD}_{3}$-groups instead of $\mathrm{CH}_{3}$ in 2-,4- and 6-position. $\frac{156}{2}$ The deuteration leads to a shift of bands, that involve the $\mathrm{C}-\mathrm{H}(\mathrm{C}-\mathrm{D})$ vibrations of $\mathrm{CH}_{3}\left(\mathrm{CD}_{3}\right)$ groups but leave the $\mathrm{C}-\mathrm{O}$ and $\mathrm{C}_{\text {ortho }}-\mathrm{C}_{\text {meta }}$ stretching unaffected. The resulting spectra are depicted in Figure 4.3. The $\nu_{7 \mathrm{a}}$ peak is found at $1244 \mathrm{~cm}^{-1}$ as it is the only signal that is not affected by deuteration in the area between 1200 and $1500 \mathrm{~cm}^{-1}$. The $\nu_{8 \mathrm{a}}$ is found at $1615 \mathrm{~cm}^{-1}$ as the only other signal not shifted upon deuteration. These two signals clearly indicate the absence 


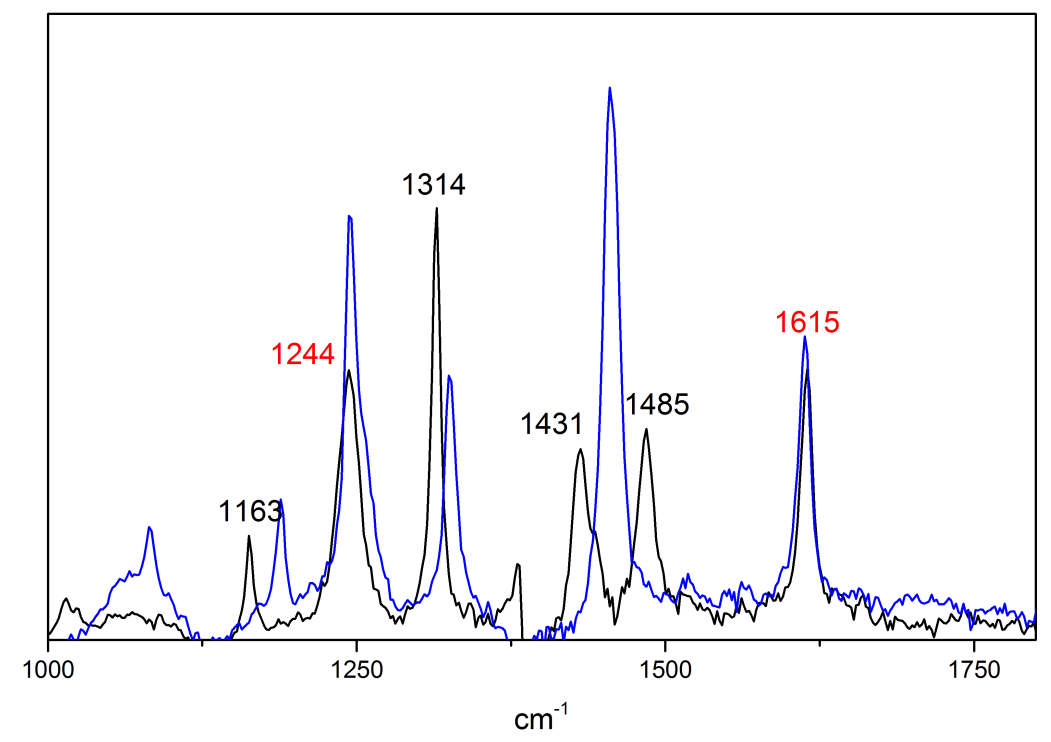

Figure 4.3 Overlay of resonance Raman spectra in frozen MeCN of the red coloured solutions containing 1 and TMP (black line) and TMP-d ${ }^{9}$ (blue line) as recorded by Prokofieva. 156 Signals that are not shifted are colored red.

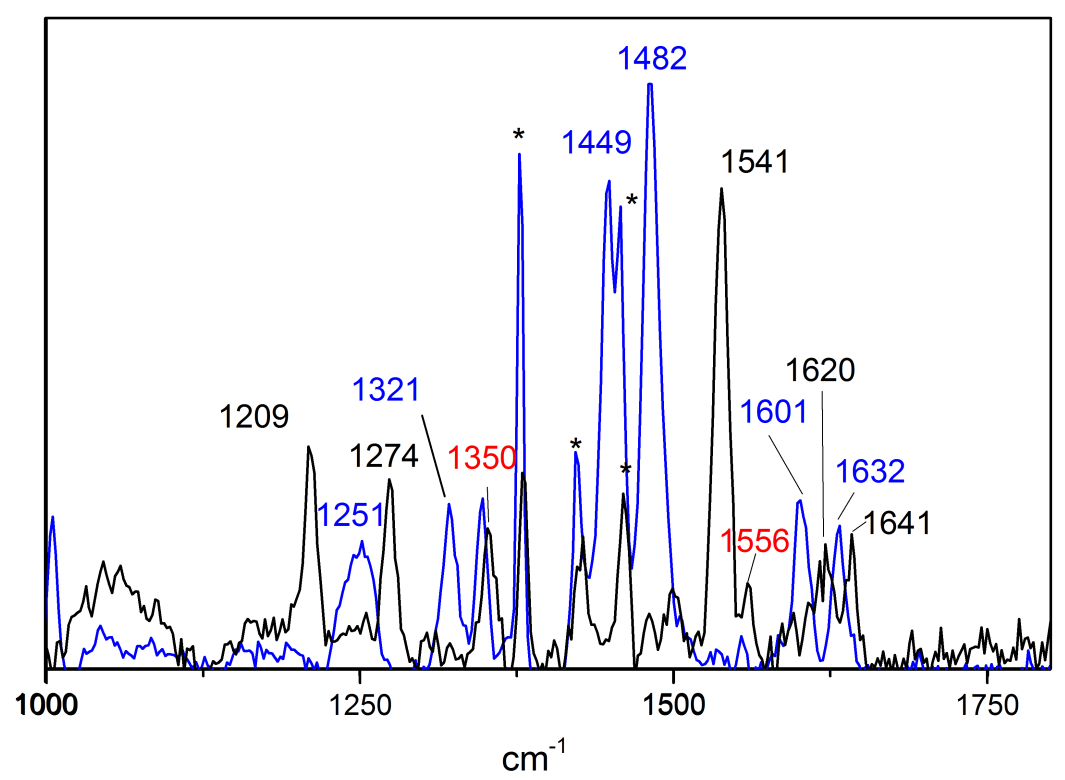

Figure 4.4 Overlay of resonance Raman spectra in frozen MeCN of the red coloured solutions containing 1 and TMBB (black line) and TMBB-d ${ }^{16}$ (blue line). Solvent signals are denoted with an asterisk. Signals that are not shifted are colored red.

of any radical character and point towards a phenoxide bound to copper(II).

The case of the TMBB adduct (recorded in this work) is evidently more complicated. Comparison between the spectrum of the deuterated and undeuterated adduct reveal two unshifted signals at $1350 \mathrm{~cm}^{-1}$ and $1556 \mathrm{~cm}^{-1}$ (Figure 4.4), which are assigned to the $\nu_{7 \mathrm{a}}$ and $\nu_{8 \mathrm{a}}$ stretching frequencies respectively. Because of this relatively low values, the presence of a copper(II) phenoxide system is more likely than a radical species. Although 
sample preparation and measurement was done at low temperatures, contamination with traces of TMSQ is possible. TMSQ shows a very similar resonance raman spectrum when measured as a solid at room temperature(Figure 4.5).

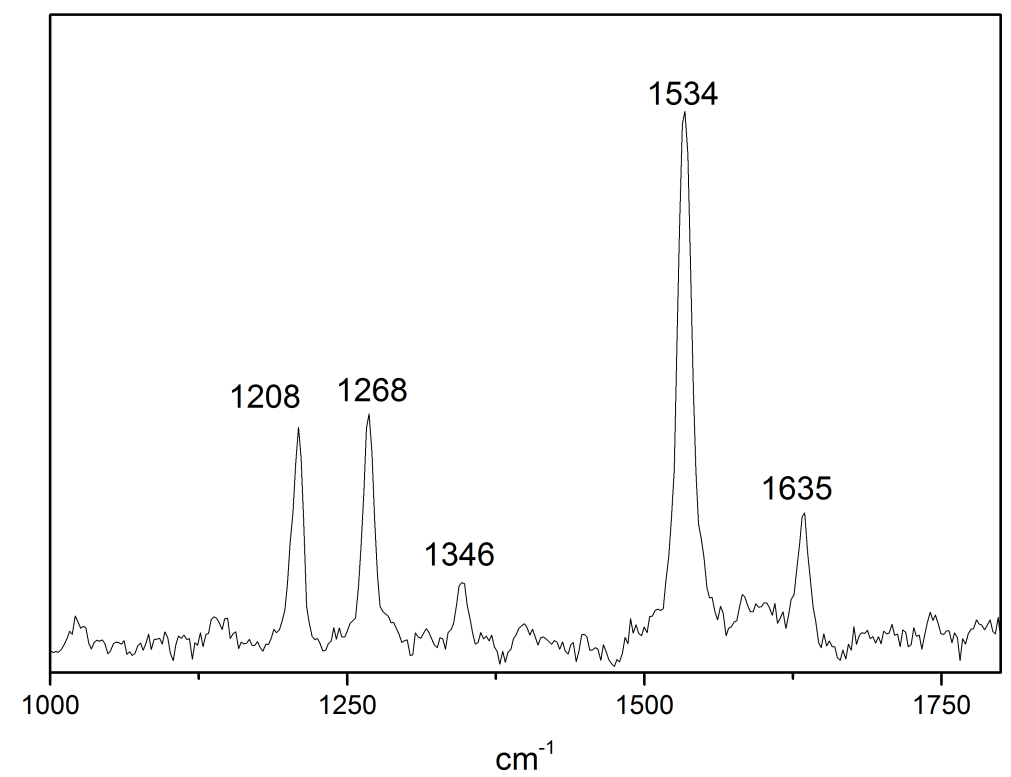

Figure 4.5 Resonance Raman spectrum of solid TMSQ.

\section{Job plot}

ProkofIEva found the binding stoichiometry between TMP and 1 to be 1:1 by using the Job plot method (Figure 4.6). It was also found out that parent phenol and $\mathbf{1}$ bind in a 2:1 ratio. $\frac{156}{15}$ This difference is caused by the steric bulk of the $\mathrm{CH}_{3}$ groups in 2- and

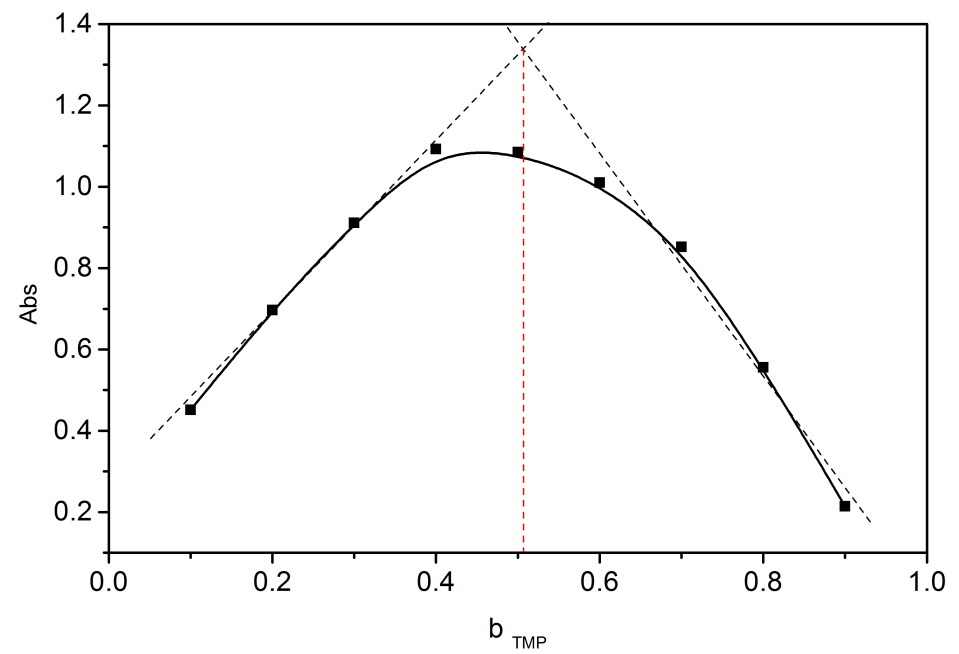

Figure 4.6 Job plot by Prokofieva to determine the binding ration between TMP and $1\left(c_{\text {total }}=5 \cdot 10^{-3} \mathrm{M}\right) .156$

6-position of TMP that prevents binding of a second phenol.

In addition to these studies, a Job plot study was undertaken in this work to determine 
the binding stoichiometry between TMBB and $\mathbf{1}$. The maximum absorption for the

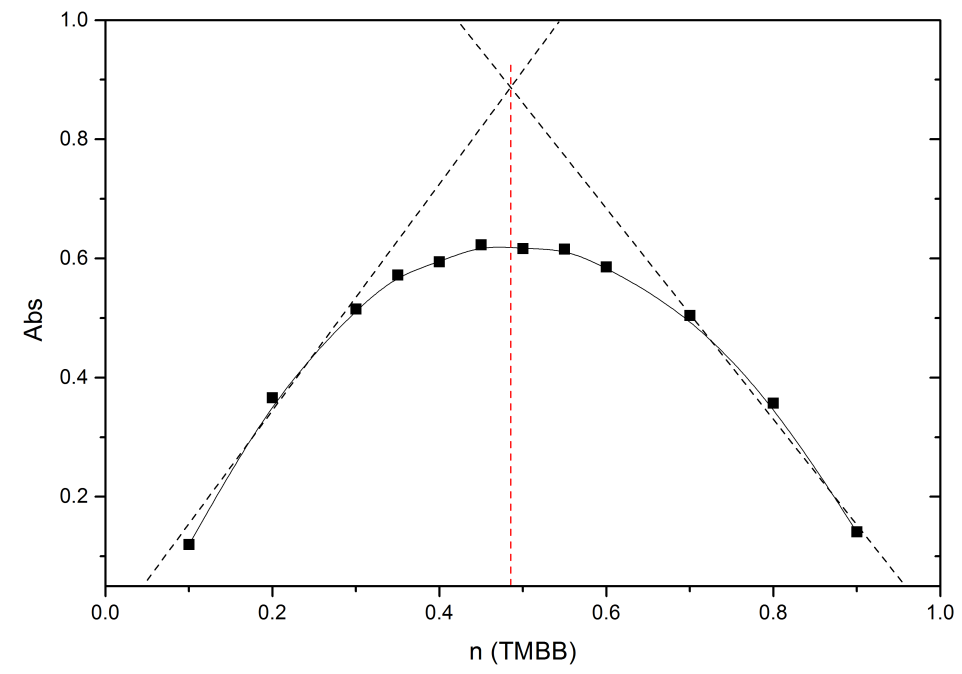

Figure 4.7 Job plot to determine the binding stoichiometry between TMBB and 1 $\left(c_{\text {total }}=10^{-3} \mathrm{M}\right)$.

LMCT band is found at a ratio of 1:1 of $\mathbf{1}$ and TMBB as can be seen in Figure 4.7.

In conclusion, TMBB and TMP both bind in a 1:1 stoichiometry to the dicopper(II) complex.

\section{Oxidation state of the catalyst after reaction with $T M B B$}

In order to understand the reaction of TMBB and $\mathbf{1}$, both reactants were dissolved in $\mathrm{MeCN}$ in a glovebox and the resulting solution was investigated with respect to the yield of TMSQ and the oxidation state of the complex.

First, UV/Vis experiments were carried out with a constant concentration of TMBB and varying equivalents of $\mathbf{1}$. Hence, a solution of TMBB in MeCN with a concentration of $10^{-3} \mathrm{~mol} \cdot \mathrm{L}^{-1}$ was prepared and divided into 6 equal portions.

Subsequently, $0.25,0.5,1,2,4$ and 6 equivalents of 1 were added and the resulting solutions were stirred overnight. Afterwards, due to the high extinction coefficient of the product, the intense yellow solutions were diluted to a concentration of $10^{-5} \mathrm{~mol} \cdot \mathrm{L}^{-1}$ in a UV/Vis cuvette, sealed with a rubber septum and placed in a UV/Vis spectrometer. As seen in Figure 4.8, addition of four equivalents of $\mathbf{1}$ are needed to fully oxidize TMBB to TMSQ and no further reaction occurs upon addition of more catalyst. The absorption increases linear with respect to the amount of $\mathbf{1}$ added until a maximum is reached after adding four equivalents.

The oxidation of TMBB to TMSQ is a four electron process and four equivalents of the dinuclear copper(II) complex are neccessary for the reaction to be complete. Therefore, it is very likely that each complex contributes one elctron to the process andforms a mixed-valent copper(I)/copper(II) species after the reaction is complete. 


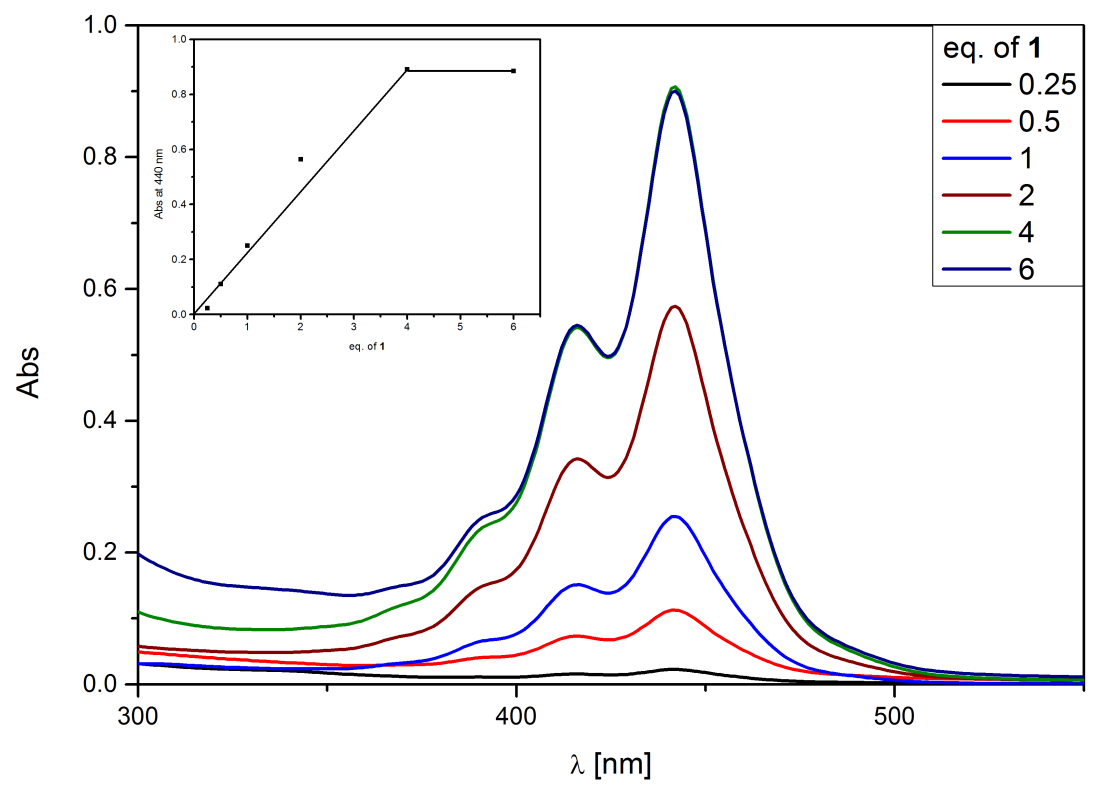

Figure 4.8 UV/Vis spectra of a solution of TMBB at roo temperature (MeCN, $c=$ $10^{-5} \mathrm{M}$ ) and increasing equivalents of $\mathbf{1}$.

To further investigate the assumption of a mixed valent species, EPR experiments were carried out. An equimolar solution of $\mathbf{1}$ and TMBB in MeCN was prepared in a glovebox and left standing over night. The solution was transferred into a Young-EPR-tube, transferred out of the glovebox and frozen in liquid nitrogen. The EPR spectrum shows the typical four-line pattern of an uncoupled mononuclear copper(II) complex. A dinuclear copper(I) complex would be EPR silent, due to the diamagnetic $d^{10}$ configuration. A dinuclear copper(II) complex would also be EPR silent at $4.2 \mathrm{~K}$ due to the thermal depopulation of energeticallly higher states at low temperatures as revealed by SQUID measurement [156] (not shown in this work). Furthermore, the resulting spectrum closely resembles that of the product of the reaction of $\mathbf{1}$ and TMP under the same conditions (Figure 4.9a)). To support these assumptions, ProkOFIEVA synthesized a heterometallic dinuclear copper(II)/zinc(II) and measured an EPR-spectrum. Again, the resulting spectrum exhibits the typical pattern for an isolated copper(II)- $d^{9}$ ion.

Providing further evidence for the existence of the proposed mixed valent valence localized species, it was possible to obtain crystals suitable for X-ray diffraction of this species by layering the reaction mixture with diethyl ether in a glovebox. A dimerized structure of the valence localized mixed valent copper(I)-copper(II) complex was observed in solid state (Figure 4.11). A slightly distorted trigonal bipyramidal coordination environment of the two copper(II) ions $(\tau=0.96)$ is provided by the two pyrazolate units, the bridging nitrogen atoms of the backbone and two imidazole moieties. The distance between the two central copper(II) ions was determined to be $3.88 \AA$ and is by $0.46 \AA$ 

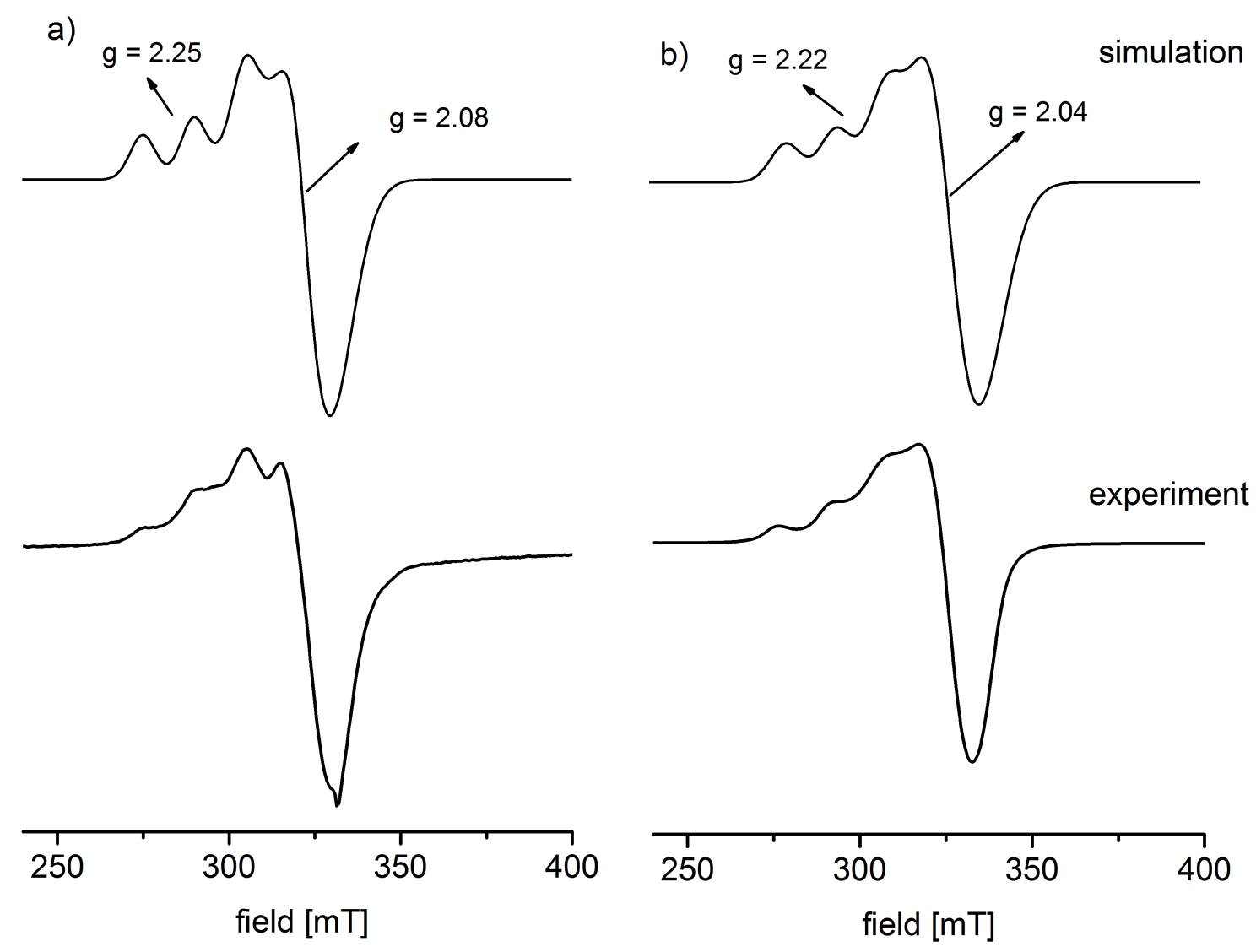

Figure 4.9 a) EPR spectrum after reaction of $\mathbf{1}\left(\mathrm{c}=10^{-3}\right)$ and 2 eq. TMP in MeCN recorded at $9.387 \mathrm{GHz}, 11 \mathrm{~K}$. Simulation gives $\mathrm{g}_{\|}=2.25\left(\mathrm{~A}=0.0153 \mathrm{~cm}^{-1}\right)$ and $\mathrm{g}_{\perp}=$ $2.08\left(\mathrm{~A}=0.0010 \mathrm{~cm}^{-1}\right)($ Additional parameters: nucleus $=\mathrm{Cu}$, total spin $=1 / 2, \mathrm{~T}=$ $11 \mathrm{~K}$, frequency $=9.387 \mathrm{GHz}$, linewidth $=8 \mathrm{mT}$, gstrain $=[0.1,0.1,0.01]) . \mathrm{b}) \mathrm{EPR}$ spectrum by Prokofieva of a heterometallic $\mathrm{Cu}(\mathrm{II}) / \mathrm{Zn}(\mathrm{II})$ complex of $\mathbf{L}^{1}$ in $\mathrm{MeCN}$ at $9.367 \mathrm{GHz}$ and $15 \mathrm{~K}$. Simulation gives $\mathrm{g}_{\|}=2.22\left(\mathrm{~A}=0.0009 \mathrm{~cm}^{-1}\right)$ and $\mathrm{g}_{\perp}=2.04$ $\left(\mathrm{A}=0.0156 \mathrm{~cm}^{-1}\right)($ Additional parameters: nucleus $=\mathrm{Cu}$, total spin $=1 / 2, \mathrm{~T}=15 \mathrm{~K}$, linewidth $=8 \mathrm{mT}$, frequency $=9.367 \mathrm{GHz}$, gstrain $=[0.05,0.15,0.05])$.

shorter than in $\mathbf{1}$. The two copper ions diverge by $12.2^{\circ}$ from the plane spanned by the 4 pyrazolate nitrogen atoms.. $\mathrm{Cu}-\mathrm{N}$ distances from the pyrazolate to the copper ions are $1.95 \AA$ and $2.03 \AA$ compared to $1.99 \AA$ and $2.02 \AA$ in $\mathbf{1}$.

The two reduced copper(I) ions point outwards from the central unit and are threefold coordinated by two histidine residues and an additional MeCN molecule. The geometry is that of a slightly distorted trigonal plane. With a distance of $3.95 \AA$ the nitrogen atom bridging the two imidazole side arms is too far away for coordination. The $\mathrm{Cu}-\mathrm{N}$ (imidazole) distances are $1.95 \AA$ and $2.00 \AA$ and therefore a little shorter than those of copper(II)-imidazole-nitrogen with $2.08 \AA$ and $2.10 \AA$ due to the lower coordination number. 


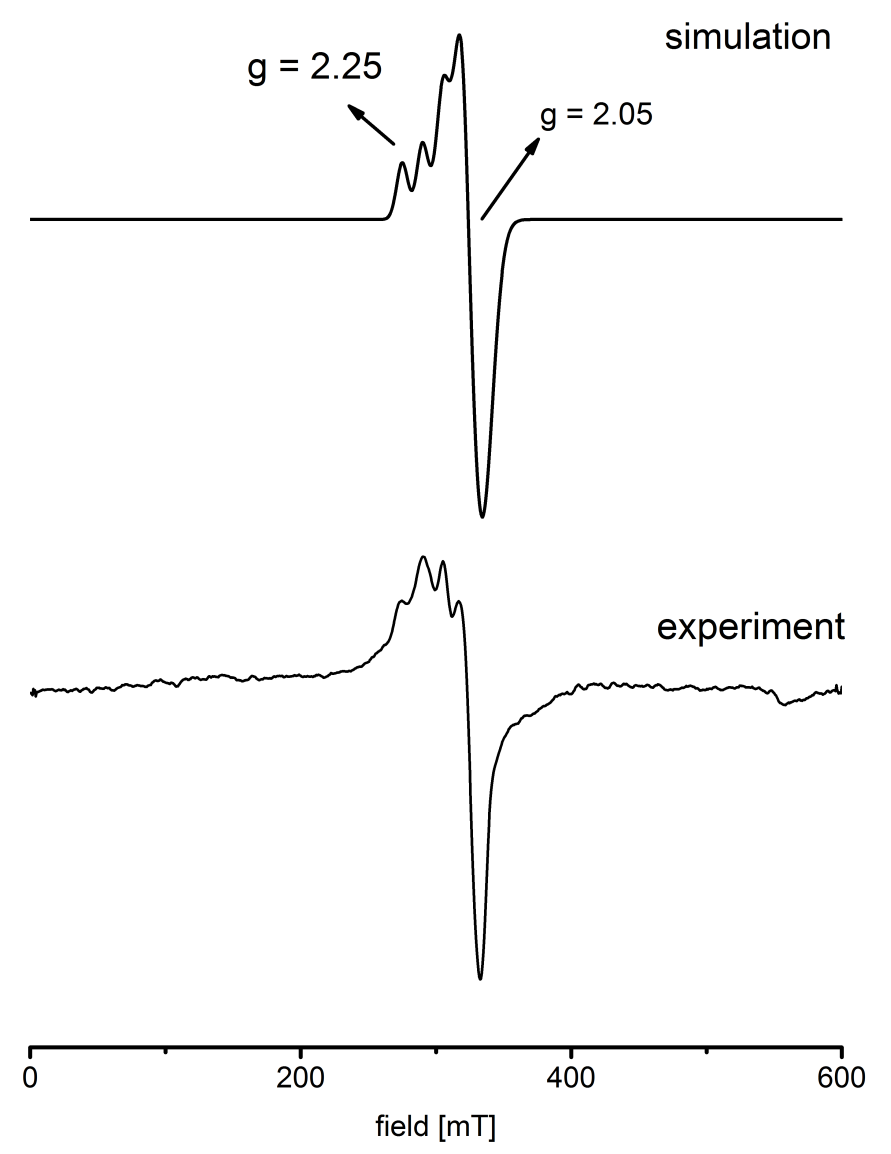

Figure 4.10 EPR spectrum, recorded at 4.2 K and 9.386 GHz, of a frozen MeCN solution $\left(c=10^{-3} \mathrm{M}\right)$ after reaction of $\mathbf{1}$ and TMBB. Simulation of the spectrum gives $\mathrm{g}_{\|}=2.25$ $\left(\mathrm{A}=0.0183 \mathrm{~cm}^{-1}\right)$ and $\mathrm{g}_{\perp}=2.05\left(\mathrm{~A}=0.0004 \mathrm{~cm}^{-1}\right)$ (simulation parameters: nucleus $=$ $\mathrm{Cu}$, total $\operatorname{spin}=1 / 2, \mathrm{~T}=4.2 \mathrm{~K}$, frequency $=9.386 \mathrm{GHz}$, linewidth $=8 \mathrm{mT}$, gstrain $=$ $[0.1,0.2,0.05])$.

\section{Magnetic measurement}

Magnetic coupling of the two copper(II) ions in $\mathbf{2}$ occurs through superexchange via the pyrazolate bridges. Therefore, the presence of two of these units should result in an increased antiferromagnetic coupling between the central copper ions. Indeed, a varibale temperature magnetic measurement of solid 2 revealed a coupling constant of $J=-88.0 \mathrm{~cm}^{-1}$ compared to $J=-35.3 \mathrm{~cm}^{-1}$ in $\mathbf{1}$.

\subsubsection{Kinetic investigations}

To further elucidate the reaction mechanism, kinetic data of the reaction between $\mathbf{1}$ and TMP have been collected under inert conditions by means of UV/Vis spectroscopy. For this purpose $\mathbf{1}$ was reacted with TMP in dfferent concentrations. The solutions were pre- 


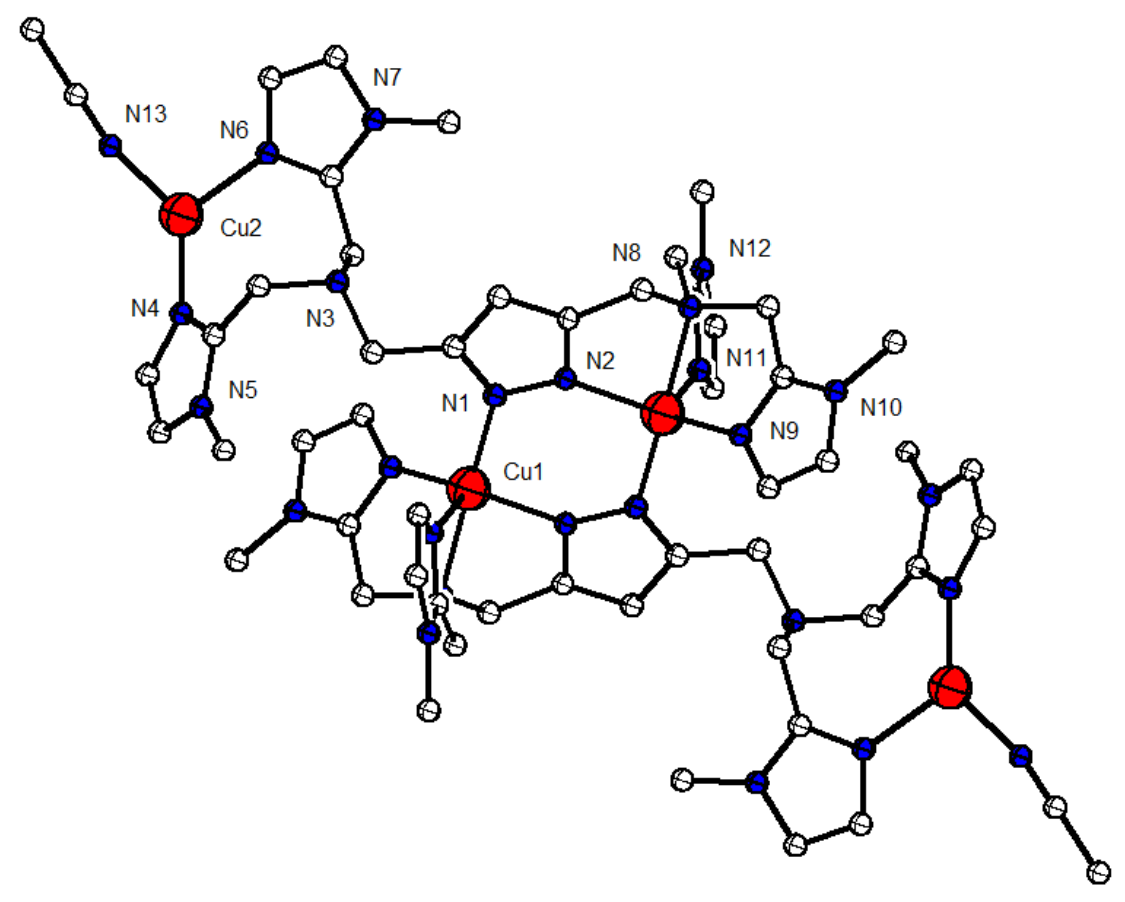

Figure 4.11 Molecular structure of the dimeric copper(I)copper(II) species 2 together with a partial labeling scheme; thermal displacement ellipsoids are drawn at $50 \%$ probability. Hydrogen atoms are omitted for clarity.

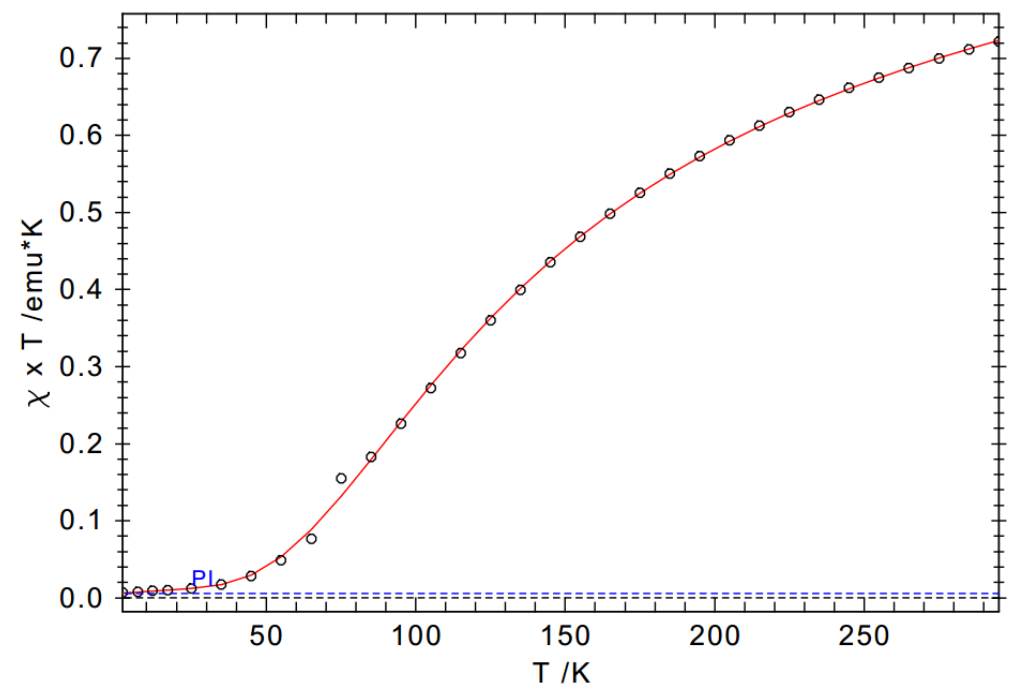

Figure 4.12 Variable temperature magnetic measurement and fit of $\mathbf{2}$.

pared in a glovebox in UV/Vis cuvettes that were sealed with a rubber septum, transferred out of the glovebox and placed in a UV/Vis-spectrometer. The decay of the LMCT band at $495 \mathrm{~nm}$, that is indicative of the copper(II)-phenolate intermediate, was monitored. Its disappearance is used as an indicator for the reduction of one copper (II)-ion to copper(I). Used combinations of concentrations can be taken from Table 4.1. Figure 4.13 shows a 
representative $\mathrm{UV} / \mathrm{V}$ is trace of the reaction. Prior to the decay of the LMCT band, the

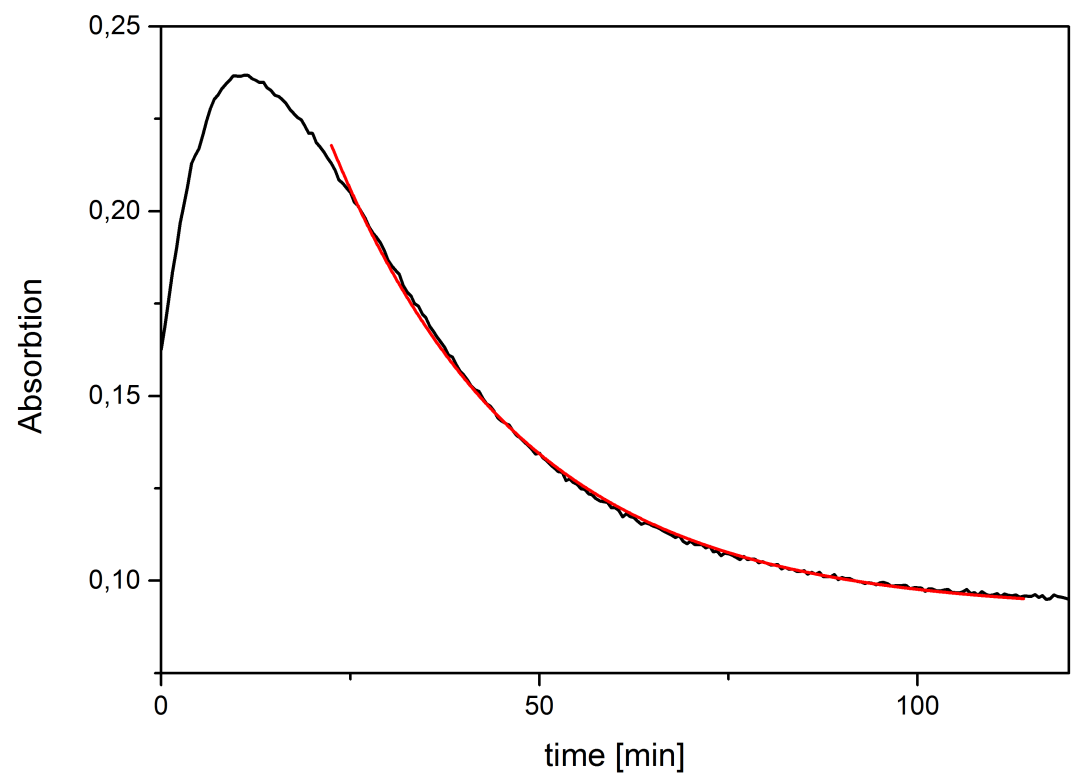

Figure 4.13 UV/Vis time trace at $495 \mathrm{~nm}$ (black line) and exponential decay fit (red line) of the reaction between $1\left(\mathrm{c}=2 \cdot 10^{-4} \mathrm{M}\right)$ and 20 equivalents of TMP $\left(\mathrm{c}=4 \cdot 10^{-3} \mathrm{M}\right)$.

binding of TMP to the complex has to take place. The first 15 to 25 minutes of the time trace represent the binding equilibrium and are not considered for the determination of the reaction rate. After the equilibrium is reached, an exponential decay is observed. The resulting reaction scheme is postulated as follows:

$$
\left[\mathbf{L}^{1} \mathrm{Cu}_{2}^{\mathrm{II}}\right]+\mathrm{TMP} \rightleftharpoons\left[\mathbf{L}^{1} \mathrm{Cu}_{2}^{\mathrm{II}}-\mathrm{TMP}\right] \rightarrow\left[\mathrm{L}^{1} \mathrm{Cu}^{\mathrm{I}} \mathrm{Cu}^{\mathrm{II}}\right]+1 / 2 \mathrm{TMBB}
$$

All reactions were monitored for 120 minutes. An exponential decay fit was applied after the reaction progressed past the binding equilibrium phase and was considered to follow a single exponential.

$$
\mathrm{y}=\mathrm{A}_{0} \cdot \exp \left(k_{o b s} \cdot t\right)+\mathrm{y}_{0}
$$

With $\mathrm{A}_{0}$ being the absorption at $t=0, t$ the time passed, $\mathrm{y}_{0}$ the offset of the $\mathrm{y}$-axis and $\mathrm{k}_{\text {obs }}$ the observed rate constant. The $\mathrm{R}^{2}$ value for each fit was $0.999-0.998$, thus good agreement of the fit with the actual data was achieved. All experiments were run twice and the average of both rate constants is shown in Table 4.1. Selected combinations of concentrations were also performed with the deuterated TMP- $\mathrm{d}^{9}$ substrate.

There is no clear trend in the observed rate constant in correlation to the concentration. Furthermore, the reaction seems to be zero order in catalyst as well as in substrate, as neither the change in catalyst concentration nor in substrate concentration changes the oberserved rate constant significantly. Neither the use of pseudo first order kinetics nor the initial rate method provided any useful results. However, as data is available over 
Table 4.1 concentrations of 1 and TMP used for kinetic measurements and obtained values for $k_{\text {obs }}$

\begin{tabular}{c|r|c|c}
\hline $\mathrm{c}(\mathbf{1})$ & $\mathrm{c}(\mathrm{TMP})$ & $k_{\text {obs }} \mathrm{TMP}\left[\cdot 10^{-2} \mathrm{~s}^{-1}\right]$ & $k_{\text {obs }} \mathrm{TMP}-\mathrm{d}^{9}\left[\cdot 10^{-2} \mathrm{~s}^{-1}\right]$ \\
\hline \multirow{4}{*}{$2 \cdot 10^{-4}$} & $5 \cdot 10^{-4}$ & 2.45 & \\
\cline { 2 - 4 } & $1 \cdot 10^{-3}$ & 2.56 & 2.46 \\
\cline { 2 - 4 } & $2 \cdot 10^{-3}$ & 3.53 & 2.06 \\
\cline { 2 - 4 } & $4 \cdot 10^{-3}$ & 4.16 & 2.41 \\
\cline { 2 - 4 } & $8 \cdot 10^{-3}$ & 3.38 & \\
\hline \multirow{5}{*}{$4 \cdot 10^{-4}$} & $1 \cdot 10^{-3}$ & 3.79 & 2.78 \\
\cline { 2 - 4 } & $2 \cdot 10^{-3}$ & 3.03 & 2.98 \\
\cline { 2 - 4 } & $4 \cdot 10^{-3}$ & 3.81 & 2.52 \\
\cline { 2 - 4 } & $8 \cdot 10^{-3}$ & 2.83 & \\
\cline { 2 - 4 } & $16 \cdot 10^{-3}$ & 4.52 & 2.78 \\
\hline \multirow{5}{*}{$8 \cdot 10^{-4}$} & $2 \cdot 10^{-3}$ & 3.05 & 2.85 \\
\cline { 2 - 4 } & $4 \cdot 10^{-3}$ & 3.60 & 2.80 \\
\cline { 2 - 4 } & $8 \cdot 10^{-3}$ & 4.25 & \\
\cline { 2 - 4 } & $16 \cdot 10^{-3}$ & 4.20 & \\
\cline { 2 - 4 } & $32 \cdot 10^{-3}$ & 4.03 & \\
\hline
\end{tabular}

the whole course of the reaction, the method of reaction progress kinetic analysis was used to determine the reaction order. ${ }^{183}$ In this method, the momentary rate constant is plotted against the momentary concentration of the observed species. In other words, the derivative of the applied fit was plotted against the momentary concentration.

The neccessesary data was obtained by converting the absorption into the concentration by the known extinction coefficient of the adduct at $495 \mathrm{~nm}$ of $\epsilon \sim 1500 \mathrm{~L} \cdot \mathrm{mol}^{-1} \cdot \mathrm{cm}^{-1}$. Deriving the fit lead to the following equation:

$$
\mathrm{r}(\mathrm{t})=\frac{\mathrm{dy}}{\mathrm{dt}}=k_{\mathrm{obs}} \cdot \mathrm{A}_{0} \cdot \exp \left(k_{\mathrm{obs}} \cdot t\right)
$$

The obtained data was plotted as described above (Figure 4.14).

When the reaction rate is plotted against the concentration at the corresponding time of the rate, a linear correlation is observed, as can be seen in Figure 4.15.

The following rate equation for the decay of the adduct was assumed:

$$
r=\frac{d P}{d t}=k \cdot\left[\mathbf{L}^{1} \mathrm{Cu}_{2}^{\mathrm{II}}\right]^{a}
$$

With $k$ being the slope of the fit and $a$ being the order of the reactant. Because the plot shows a linear correlation between the reaction rate and the concentration of the adduct, the reaction is first order in this adduct.

The same result is obtained for all investigated combinations of concentration.

kinetic isotope effect

When the obtained $\mathrm{k}_{\mathrm{obs}}$-values for the undeuterated and the deuterated substrate are 

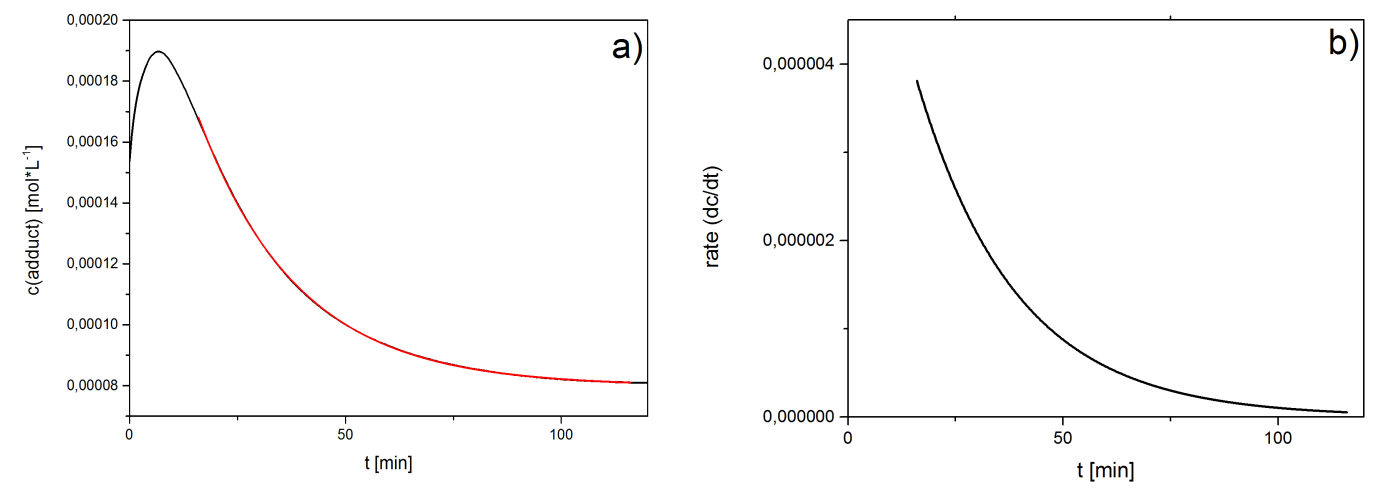

Figure 4.14 a) time dependent concentration profile (black line) and exponential fit (red line). b) time dependent reaction rate of fitted data vs time

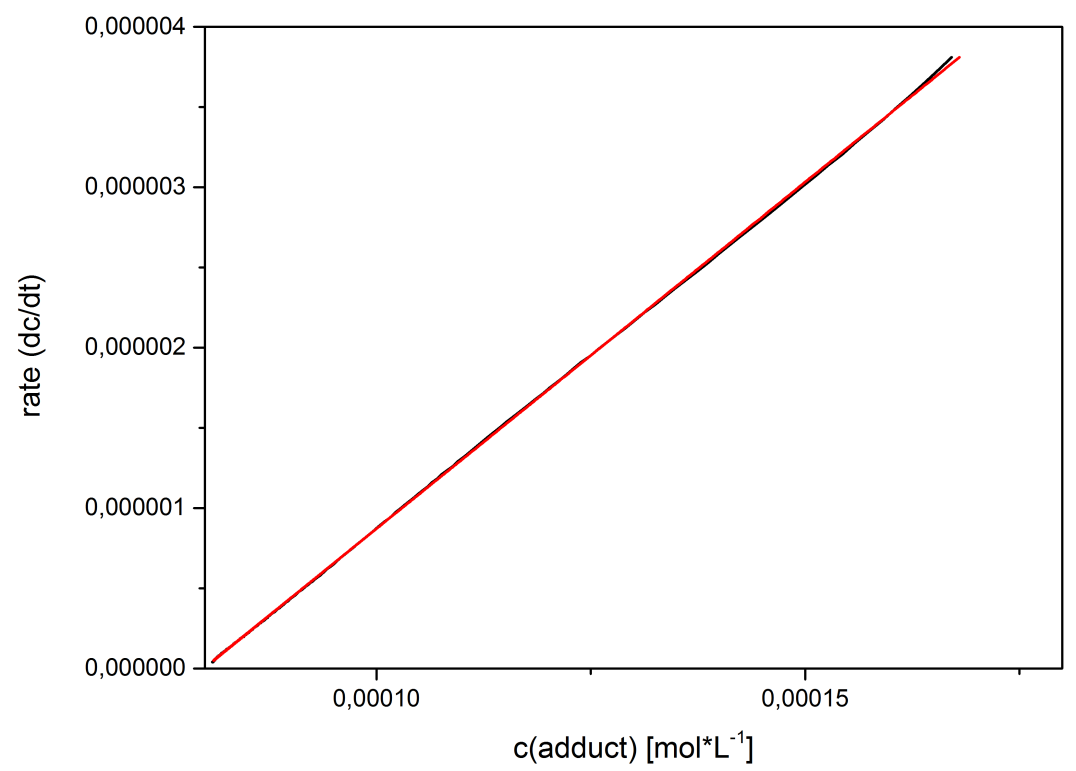

Figure 4.15 back line) rate of collected data vs. momentary concentration red line) rate of fitted data vs. momentary concentration.

compared, an average kinetic isotope effect of $\mathrm{k}_{\mathrm{H} / \mathrm{D}}=1.3$ is observed. Although this data lies within the margin of error of $1.5 \cdot 10^{-2} \mathrm{~s}^{-1}$ for the single reactions and could therefore be purely coincidental, there does seem to be a trend towards slower reaction rates for the deuterated substrate. The reaction does not involve $\mathrm{C}-\mathrm{H}$ bond breaking as the rate determining step.

\subsubsection{Reoxidation of the mixed valent copper(I)copper(II) species by molecular dioxygen}

The terminal oxidant of the oxidative $\mathrm{C}-\mathrm{C}$ coupling of 2,4,6-trimethyl phenol catalyzed by dinuclear compartmental pyrazolate bridged complex $\mathbf{1}$ is dioxygen. The final step in the 
catalytic cycle is the reoxidation of the mixed valent copper(I)-copper(II) intermediate back to its initial dicopper(II) state.

Crucial information about the nature of the reaction can be gathered by determining the whereabouts of the abstracted para- $\mathrm{C}-\mathrm{H}$ proton and the fate of dioxygen involved in the reoxidation of the mixed valent species. The possibilities for the reduced oxygen species is either superoxide, hydrogen peroxide or water.

First, the reaction solution was tested for superoxide with nitroblue-tetrazolium chloride (NBT), that shows a strong absorption band at $620 \mathrm{~nm}$ when reacted with superoxide. 184 To exclude superoxide dismutase activity of $\mathbf{1}, \mathrm{UV} / \mathrm{V}$ is experiments with a fixed amount of $\mathrm{KO}_{2}$ and $\mathrm{ABTS}$ in dry DMSO and increasing amounts of $\mathbf{1}$ were carried out prior to the actual test. The results are depicted in Figure 4.16. Though varying in absorption, there is no obvious influence of the presence of the catalyst on the recorded absorption and therefore no superoxide dismutase activity of $\mathbf{1}$ is observed. If the complex was able to disproportionate superoxide, a decrease in absorption with increasing amount of complex would be observed.

When superoxide dismutase activity was excluded, NBT was added to the reaction mixture. In the recorded UV/Vis spectra no increase in absorption at $620 \mathrm{~nm}$ was observed and therefore it is unlikely for superoxide to be formed during the process of reoxidation of the dicopper center.

After excluding superoxide as the reoxidation product, an ABTS essay was carried out to

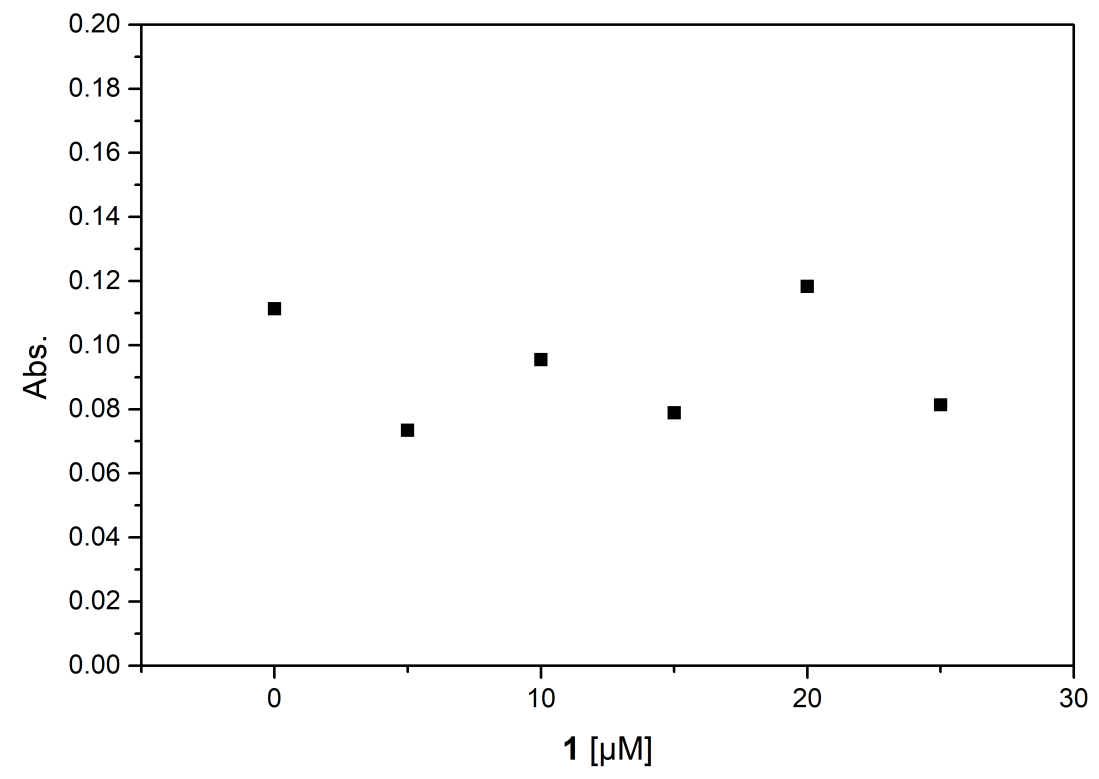

Figure 4.16 UV/Vis absorption at $620 \mathrm{~nm}$ of different solutions containing $\mathrm{KO}_{2}$ in dry DMSO and increasing concentrations of 1 .

detect hydrogen peroxide. Upon reaction with $\mathrm{H}_{2} \mathrm{O}_{2}$, ABTS shows an absorption band at $420 \mathrm{~nm}$. 185 First, $\mathbf{1}$ was tested for catalase activity, to exclude the possibility that evolving hydrogen peroxide is undetectable due to the respective catalytic activity of the 
complex. For this purpose, a solution containing an excess of ABTS was placed in an $\mathrm{UV} /$ Vis-spectrometer and $\mathrm{H}_{2} \mathrm{O}_{2}$ was added under stirring and the time trace at $420 \mathrm{~nm}$ was recorded. In a second experiment, 1 was added prior to $\mathrm{H}_{2} \mathrm{O}_{2}$ addition and the time trace recorded again. The resulting spectra are depicted in Figure 4.17. The two traces are almost identical in their time evoution and result in the same maximum absorption, showing that there is no influence of the catalyst on the reaction of ABTS with hydrogen peroxide and therefore no catalase activity. When ABTS was added to the reaction of 1 with TMP, no signal for oxidized ABTS was detectable by UV/Vis spectroscopy. The absence of a band at $420 \mathrm{~nm}$ clearly indicates the absence of hydrogen peroxide. Since the complex does not exhibit catalase activity, this means that there is no hydrogen peroxide formation during the reaction.

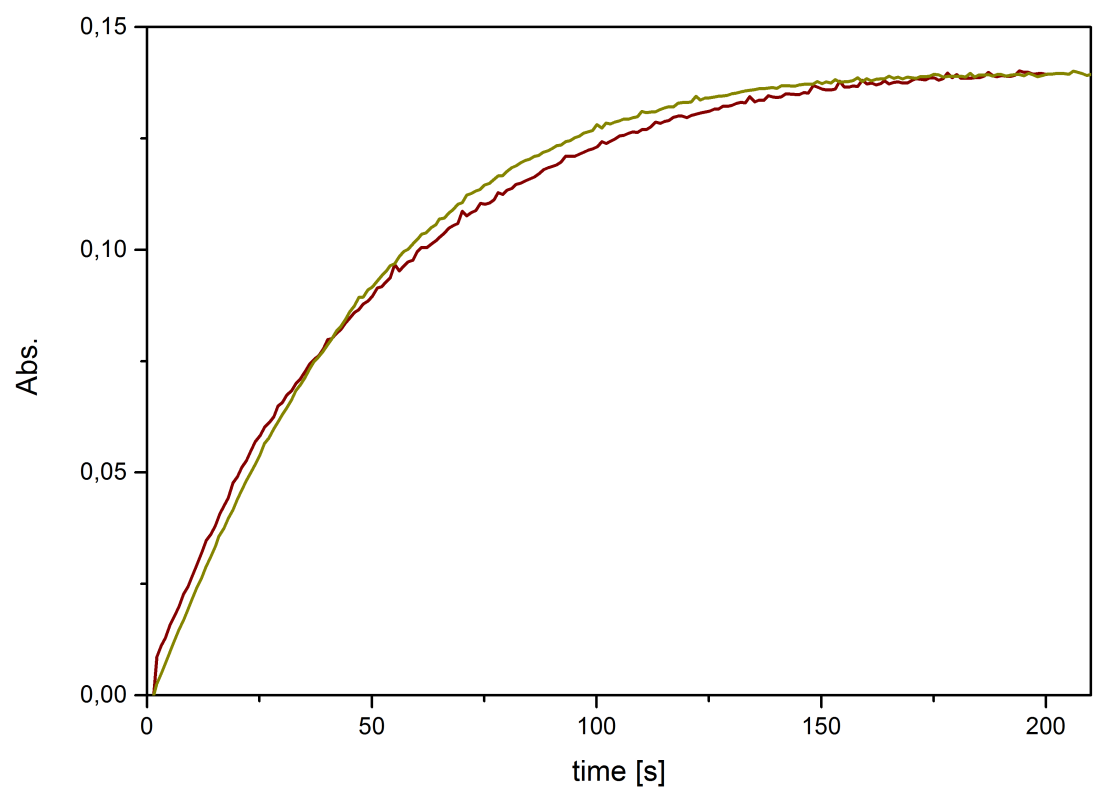

Figure 4.17 UV/Vis time trace at $420 \mathrm{~nm}$ of the reaction of $\mathrm{H}_{2} \mathrm{O}_{2}$ with ABTS in the absence (yellow line) and presence of $\mathbf{1}$ (red line).

So far, water is the most likely product of reoxidation as there is no evidene of superoxide or peroxide in the reaction mixture. To further investigate the involvement of dioxygen in the reaction and its fate as the terminal oxidant, oxygen uptake of the reaction mixture in a sealed vessel and a defined amount of dioxygen was investigated with a CLARK-electrode setup. First, a calibration curve was obtained by flushing the reaction cell with nitrogen and adding $2 \mathrm{~mL}$ of degassed MeCN and subsequently $250 \mu \mathrm{L}$ of dried dioxygen and referencing the electrode potential to the resulting concentration in the cell. Afterwards, $\mathbf{1}$ $(10 \mu \mathrm{mol})$ was placed in the cell as a solid, which was then flushed with nitrogen. The complex was dissolved in degassed MeCN and an equal amount of TMP in degassd MeCN was added to a total volume of $2 \mathrm{~mL}$. Subsequently an excess of dioxygen has been added 
to the reaction vessel and the reaction was monitored for 17 hours. The experimental setup yields the oxygen concentration over time, which was transformed into the total amount by multiplying this concentration with the volume of the reaction vessel. The

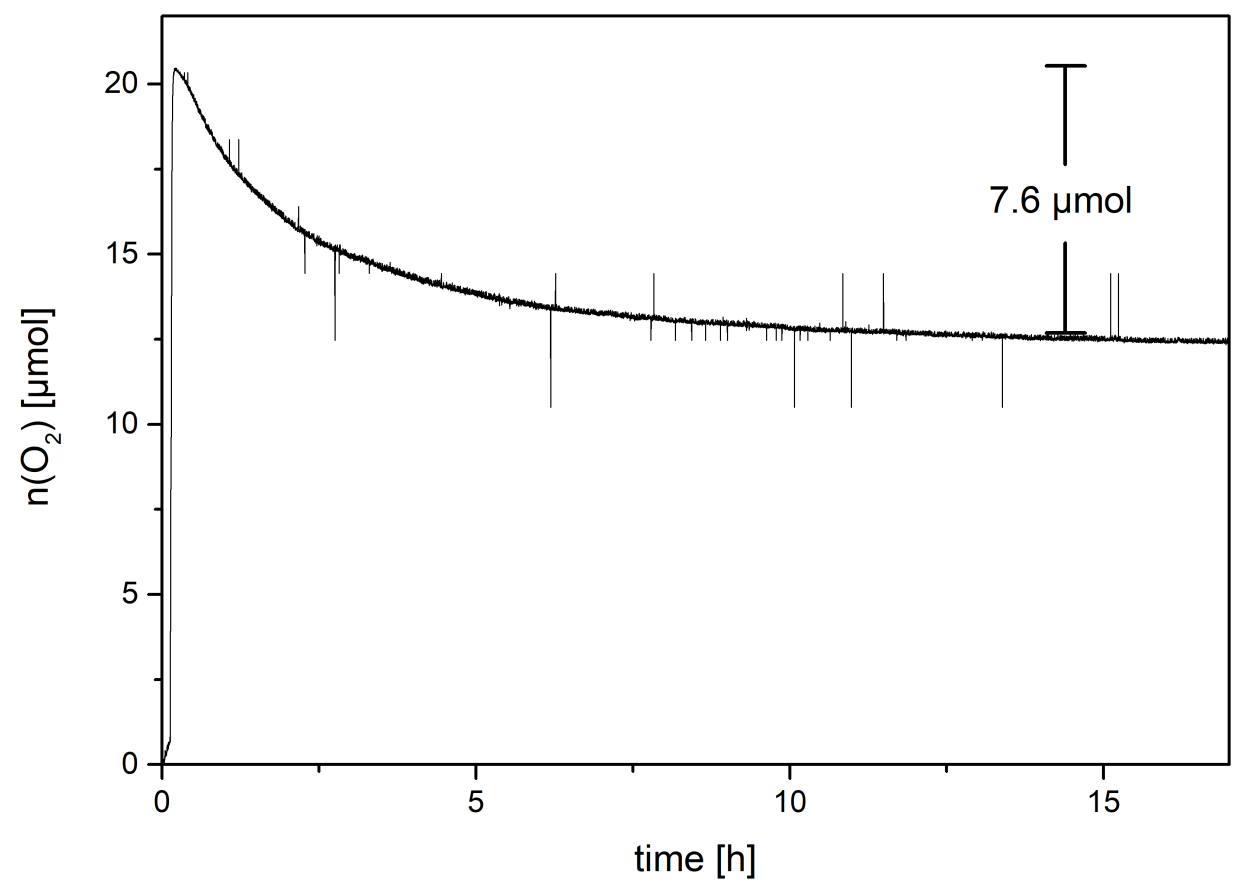

Figure 4.18 Time trace of amount of molecular dioxygen present in a sealed reaction vessel containing $10 \mu \mathrm{mol}$ of $\mathbf{1}$ and TMP dissolved in $2 \mathrm{~mL}$ of degassed MeCN.

total change in the amount of molecular dioxygen over time can be seen in Figure 4.18. After an initial equilibrium within the solution, $500 \mu \mathrm{L}$ of dioxygen were added and accordingly a strong increase in the amount of dioxygen is observed. Over time, the amount decreases in an exponential manner and reaches a plateau after roughly 16 hours. The amount of dioxygen consumed totals $7.6 \mu \mathrm{mol}$, resulting in a stoichiometry of TMP: $\mathrm{O}_{2}$ of 4:3. As mentioned earlier, the reaction of two molecules of TMP to give TMSQ is a $6 e^{-} / 6 \mathrm{H}^{+}$process, so three electrons per molecule of TMP are removed. In the end, these electrons are taken up by the dioxygen present in the vessel. Dioxygen can take up up to four electrons to form water in the presence of protons. Given the stoichiometry, it is evident that the aforementioned case is the one at hand. Three molecues of dioxygen can take up twelve electrons that are donated by 4 molecules of TMP, resulting in the observed 4:3 stoichiometry.

While three molecules of dioxygen are reduced to water, a demand for twelve protons arises to form six water molecules. This demand can for one part be satisfied by eight abstracted para- $\mathrm{C}-\mathrm{H}_{3}$ protons of the four TMP molecules, for another part by four $\mathrm{O}-\mathrm{H}$ protons. Unfortunately, it was not possible to experimentally detect the presence of water as of yet. 
<smiles>CC1=CC(=CC=C2C=C(C)C(=O)C(C)=C2)C=C(C)C1=O</smiles>

Scheme 4.1 Stoichiometry of the oxidative para-C-C coupling and subsequent oxidation of TMP mediated by $\mathbf{1}$.

\subsubsection{Proposed reaction mechanism}

Taken all the previous results into account a catalytic cycle is proposed (Figure 4.19).

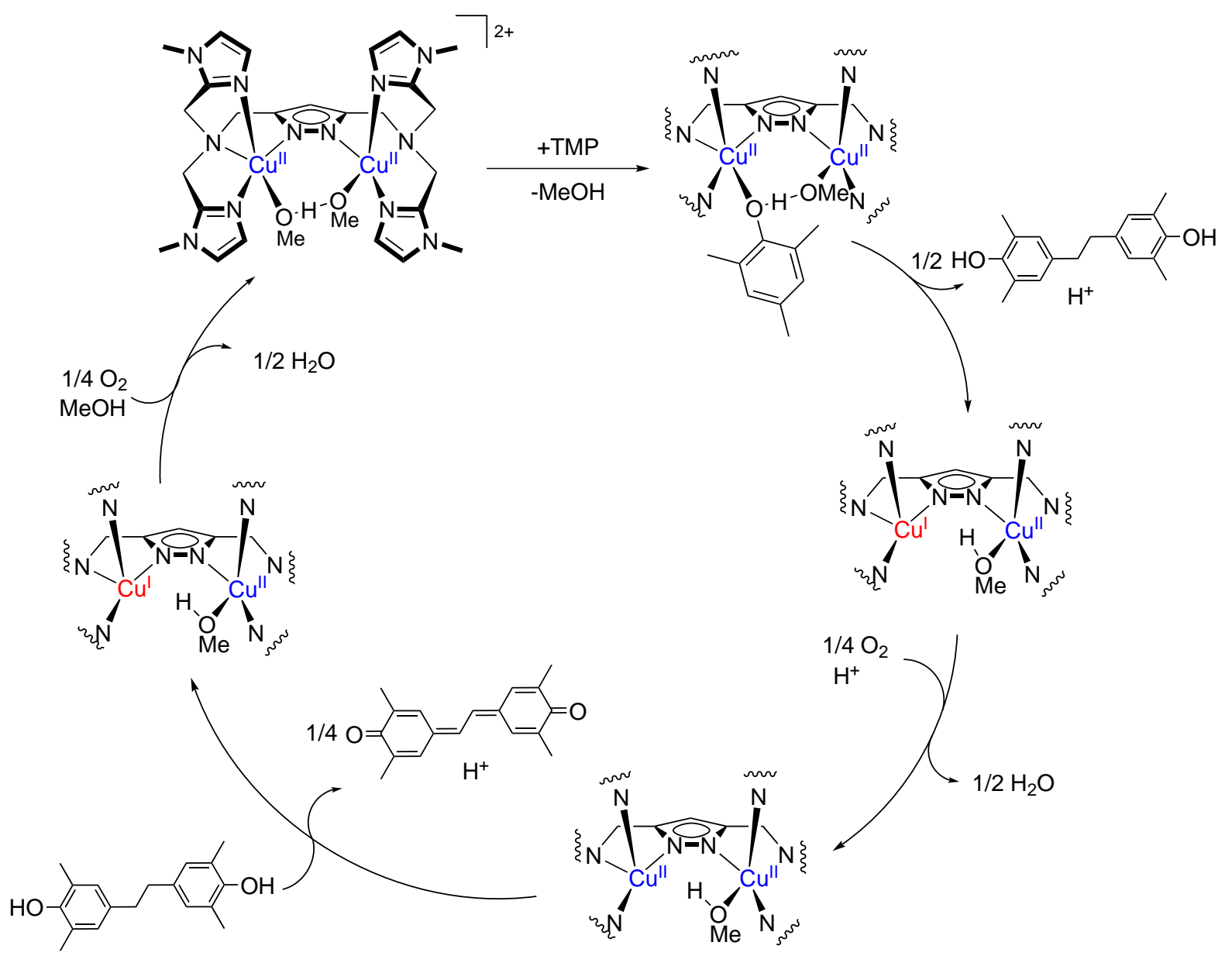

Figure 4.19 Postulated catalytic cycle of the oxidation of TMP mediated by $\mathbf{1}$.

\section{1) Binding of $T M P$}

Coordination of a single TMP molecule to one of the copper(II) ions of $\mathbf{1}$ was proven by $\mathrm{UV} / \mathrm{Vis}$, resonance raman spectroscopy and x-ray christallography with a model substrate. The coordination of TMP is indicated by an intense LMCT band at $495 \mathrm{~nm}$.

\section{2) Oxidation of $T M P$}

When the reaction is carried out under anaerobic conditions, the colour of the mixture 
fades over time, as the reaction progresses. Since the colour arises from the aforementioned LMCT transition, a decay of this band corresponds to a subsequent formation of the proposed mixed valent complex.

While its widely believed that the reaction occurs via a radical mechanism, there was no spectroscopic evidence for this behaviour. However, it is possible that the intermediate remains in a polar state and only undergoes radical coupling upon collision with another molecule of its type. However, the observed first order kinetic for the oxidative coupling of TMP is counterinidicating this assumption.

Nevertheless, the complex is capable of generating the very stable 2,4,6-tritertbutyl phenol radical, while this remains the only observed radical species.

\section{3) Formation of $T M B B$}

The first organic product of the reaction is TMBB in $100 \%$ yield with respect to $\mathbf{1}$, as proven by ${ }^{1} \mathrm{H}-\mathrm{NMR}$ spectroscopy. In the absence of dicopper(II) complex $\mathbf{1}$, TMBB is stable as a solid and in solution under ambient conditions. Under inert conditions, the reaction is halted at this stage and no further reaction occurs, leaving the complex in its mixed valent copper(I)copper(II) state as proven by EPR spectoscopy and a crystallized dimerized $\mathrm{Cu}^{\mathrm{I}} \mathrm{Cu}^{\mathrm{II}}$-species.

\section{4) Reoxidation of the copper(I)/copper(II) intermediate}

It was experimentally shown that the mixed valent intermediate does not generate superoxide upon reoxidation, nor does $\mathbf{1}$ show SOD activity that would interfere with the detection of superoxide. Hydrogen peroxide was also not detected and the complex does not exhibit catalase activity that would result in a false negative for the presence of hydrogen peroxide.

In dioxygen uptake experiments using a CLARK electrode setup, a stoichiometry of oxygen:TMP of 3:4 was found, indicating the uptake of four electrons per oxygen molecule, which results in the reduction of dioxygen to water. Together with the absence of superoxide and hydrogen peroxide, water is the most likely product of reoxidation, while the required protons for the formation of water stem from the oxidized substrate. Mechanistic details of the reoxidation of the $\mathrm{Cu}^{\mathrm{I}} \mathrm{Cu}^{\mathrm{II}}$ intermediate by $\mathrm{O}_{2}$ remain unknown.

\section{5) Binding and Oxidation of TMP or TMBB}

After the complex is reoxidized, it can bind either another TMP molecule or the newly formed TMBB. If TMBB is bound, it is further oxidied in a $4 e^{-} / 4 \mathrm{H}^{+}$process mediated by the dicopper(II) catalyst, to give the mixed valent copper(I)/copper(II) species and TMSQ, as was shown by means of UV/Vis and EPR spectrocopy. Job plot studies confirm a 1:1 stoichiometry of TMBB and $\mathbf{1}$, also epxlaining the one electron oxidation 
of the complex, as only one of the two copper ions is coordinated by a substrate. TMBB is readily oxidized to TMSQ under mild conditions which is most likely the reason, why no intermediate product is observed.

6) Reoxidation

After the reaction with TMBB, the catalyst is found again in a mixed valent copper(I)copper(II) state and is reoxidized to its initial dicopper(II) state by molecular dioxygen. This final step closes the reaction cycle. 


\subsection{Functionalization of TMP in para position}

Besides the $\mathrm{C}-\mathrm{C}$ coupling of TMP in para-position it is also possible to catalytically functionalize TMP in para-position. To gain access to a wider variety of products starting from TMP, it is possible to expand the scope of reactions simply by varying the solvent in which the transformation is carried out. Furthermore, it is possibile to selectively choose the kind of product by choice of the reaction time, as the first product reacts further over time.

\subsubsection{Synthesis of 2,6-dimethyl-4-methylmethoxy-phenol}

When the reaction of TMP and $\mathbf{1}$ is carried out in presence of methanol, 2,6-dimethyl4-methoxymethyl-phenol (MDP) is obtained as the main product. The reaction was carried out under ambient conditions in a mixture of $\mathrm{MeOH} / \mathrm{MeCN} 1 / 3$ with $5 \%$ catalyst loading. Determination of the product was done by filtration of the reaction mixture through silica, removing the solvent under reduced pressure and measuring ${ }^{1} \mathrm{H}$ - and ${ }^{13} \mathrm{C}\{\mathrm{H}\}$-NMR spectra. The conversion was determined by comparing the integrals of the aromatic protons of starting material and product. As can be seen in Figure 4.20 TMP is very slowly converted into MDP with about 45 hours to convert half of the subtsrate. While being very slow at ambient conditions, the conversion is clean and does not generate any kind of side product in amounts detectable by ${ }^{1} \mathrm{H}-\mathrm{NMR}$ spectroscopy. There seems to be a long initiation phase after which the progress is sped up. This behaviour cannot be explained at the moment. The progress of the reaction can be sped

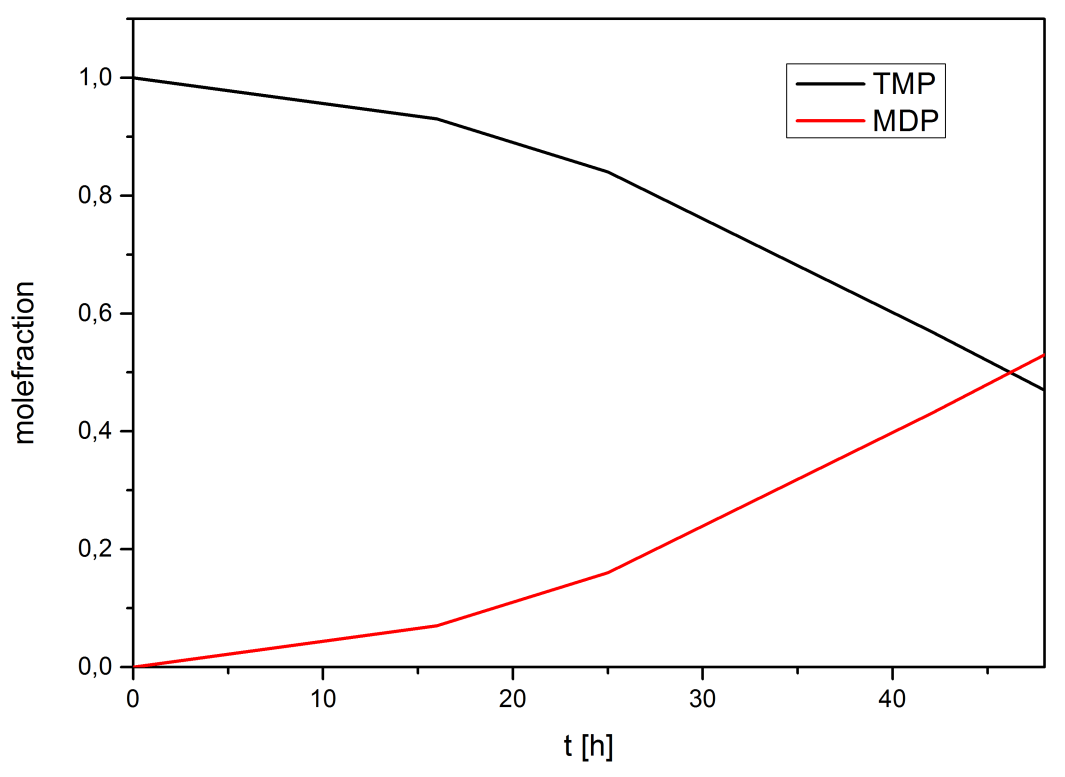

Figure 4.20 Time profile of the conversion of TMP to MDP in MeCN/MeOH 3/1 under ambient conditions. 
up by increasing the temperature but at the cost of generating unwanted side products as well as a higher oxidized product. With this in mind, it was decided not to use elevated temperatures for the reaction but to prefer a clean conversion at ambient temperatures. When the reaction was carried out in the absence of oxygen, a substoichiometric amount of MDP with respect to the catalyst was observed.

In order to determine the origin of the methoxy grop, deuterated methanol was used for the reaction and the obtained product analyzed by ${ }^{1} \mathrm{H}-\mathrm{NMR}$ spectroscopy. The complete absence of the methoxy-signals in the ${ }^{1} \mathrm{H}-\mathrm{NMR}$ spectrum revealed the solvent as the source of the methoxy group. Whether this methanol is bound in the complex or freely dissolved could not be clearified.

EPR spectra of a MeCN/MeOH-solution containing $\mathbf{1}$ and TMP were recorded at $170 \mathrm{~K}$
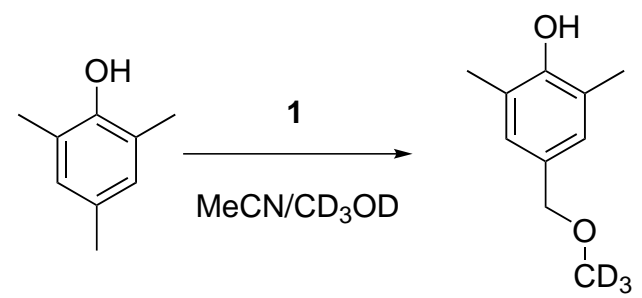

Scheme 4.2 Reaction scheme of TMP with deuterated methanol catalyzed by $\mathbf{1}$.

and $6 \mathrm{~K}$ in order to determine the oxidation state of the complex after the reaction (Figure 4.21).

The spectrum recorded at $170 \mathrm{~K}$ shows a half field signal at around $152 \mathrm{mT}$ and the main signal at $310 \mathrm{mT}$ with shoulders at $270 \mathrm{mT}$ and $380 \mathrm{mT}$. The $\mathrm{g}_{\|}$and the $\mathrm{g}_{\perp}$ value are both 2.14 and the corresponding A-values are 0.0320 and $0.0021 \mathrm{~cm}^{-1}$ respectively. The $\mathrm{g}$-value of the half field signal is 4.33 . The same values were obtained for $\mathbf{1}$. Therefore, the observed species is most likely the unreacted dicopper(II) complex. This observation is in agreement with the substoichiometric yield of MDP in the absence of oxygen.

The spectrum recorded at $6 \mathrm{~K}$ does not exhibit a half field signal. The main signal is found at $320 \mathrm{mT}$ and exhibits a $\mathrm{g}_{\perp}$-value of 2.07 and and $\mathrm{A}$-value of $0.0018 \mathrm{~cm}^{-1}$. The $\mathrm{g}_{\|}$value was found to be 2.19 with an A-value of $0.0032 \mathrm{~cm}^{-1}$. The unusual pattern may be caused by a further shortening of the typical four line pattern, as observed previously. Anyhow, the presence of a signal in agreement to that of a monouclear copper complex indicates the presence of a mixed valent state as observed for the $\mathrm{C}-\mathrm{C}$ coupling reaction, albeit in small concentration.

In conclusion, only a fraction of $\mathbf{1}$ actually undergoes a reaction with the substrate in the employed solvent mixture, most likely due to a shift of the binding equilibrium towards methanol due to ist high concentration. When the complex undergoes a reaction, it oxidizes the substrate by one electron and is reduced to the previously observed 
a)
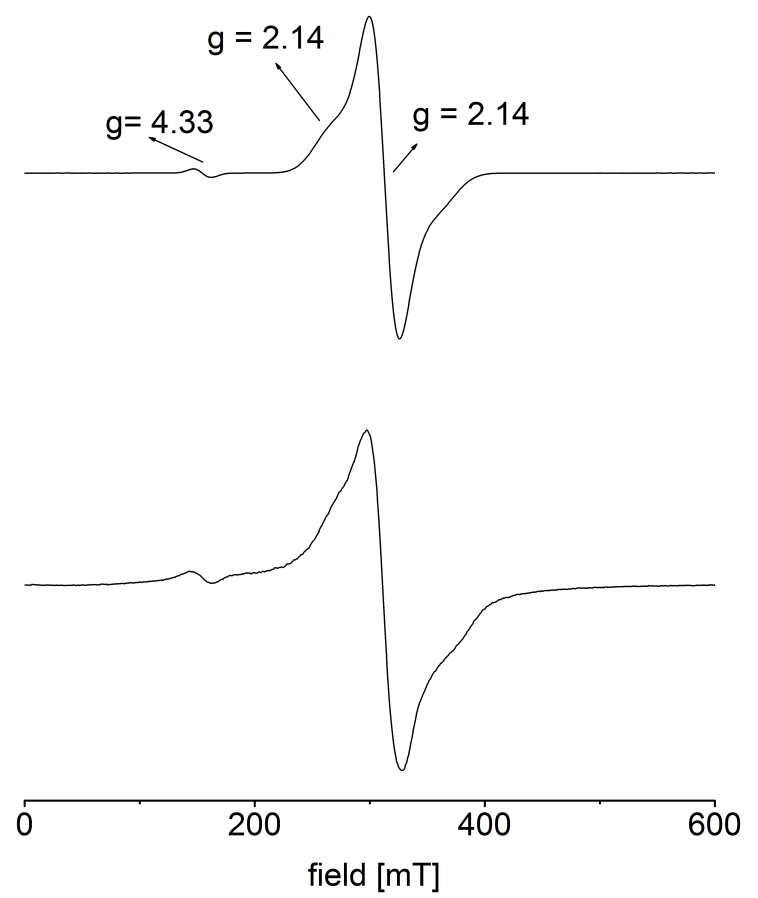

b)

simulation
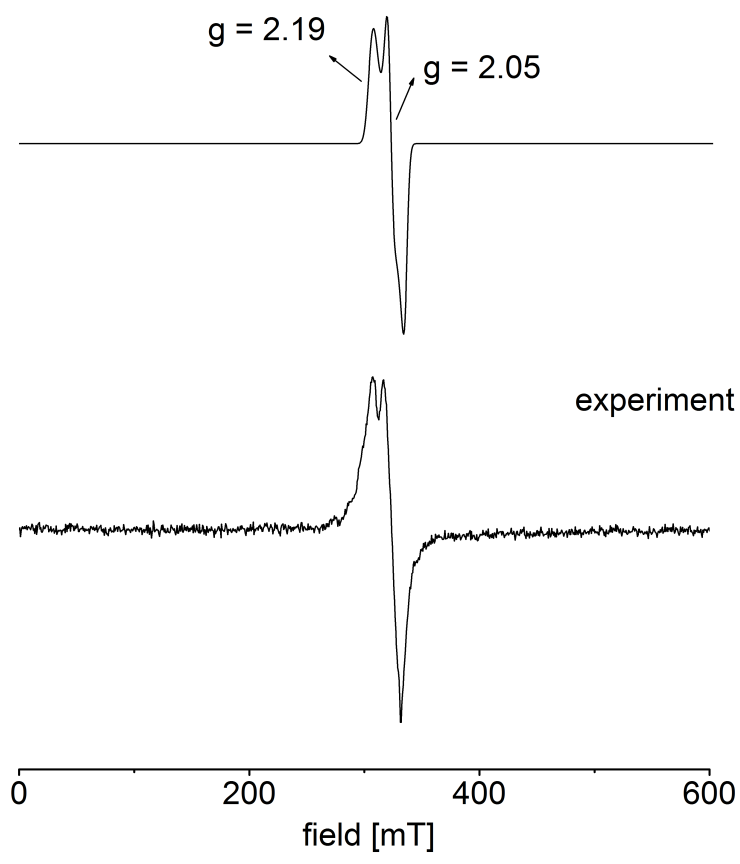

Figure 4.21 a) EPR spectrum of a MeCN/MeOH solution containing 1 and TMP after stirring over night in a glovebox, recorded at $170 \mathrm{~K}$ and $9.429 \mathrm{GHz}$ (simulation parameters: Nucleus $=\mathrm{Cu}, \mathrm{Cu}$, total spin $=1, \mathrm{~T}=170 \mathrm{~K}$, frequency $=9.429 \mathrm{GHz}$, linewidth $=8$ $\mathrm{mT}, \mathrm{g}_{\|}=\mathrm{g}_{\perp}=2.14, \mathrm{~A}=0.0290 \mathrm{~cm}^{-1}$, gstrain $\left.=[0.3,0.05,0.18]\right)$. b) EPR spectrum of the same solution recorded at $6 \mathrm{~K}$ (simulation parameters: nucleues $=\mathrm{Cu}$, total spin $=1 / 2, \mathrm{~T}=6 \mathrm{~K}$, frequency $=9.368 \mathrm{GHz}$, linewidth $=6 \mathrm{mT}, \mathrm{g}_{\|}=2.19 \mathrm{~g}_{\perp}=2.05, \mathrm{~A}_{\|}$ $=0.0029 \mathrm{~cm}^{-1}, \mathrm{~A}_{\perp}=0.0027 \mathrm{~cm}^{-1}$, gstrain $\left.=[0.1,0,0.06]\right)$.

copper(I)/copper(II) state.

\subsubsection{Synthesis of 2,6-dimethyl-4-hydroxymethyl-phenol and sub- sequent oxidation}

Another possibility is to carry out the reaction in the presence of water to obtain 2,6-dimethyl-4-hydroxymethyl phenol (HMP). The reaction was carried out in acetone/MeCN/water $(2 / 2 / 1)$ with $5 \%$ catalyst loading under ambient conditions. The ratio between $\mathrm{MeCN}$ and acetone determines the rate and the purity of the conversion. A higher amount of acetone leads to a conversion without side products but to significantly longer reaction time. The employed solvent mxiture was determined to be the best compromise between reaction rate and clean conversion.

A two step process is observed for the reaction under the given conditions. TMP is almost completely transformed to HMP within the first 15 hours. When the concentrations of TMP and HMP are roughly equal, a second product emerges. The newly generated HMP is slowly converted to 4-hydroxy-3,5-dimethyl benzaldehyde (HDB), reaching half 


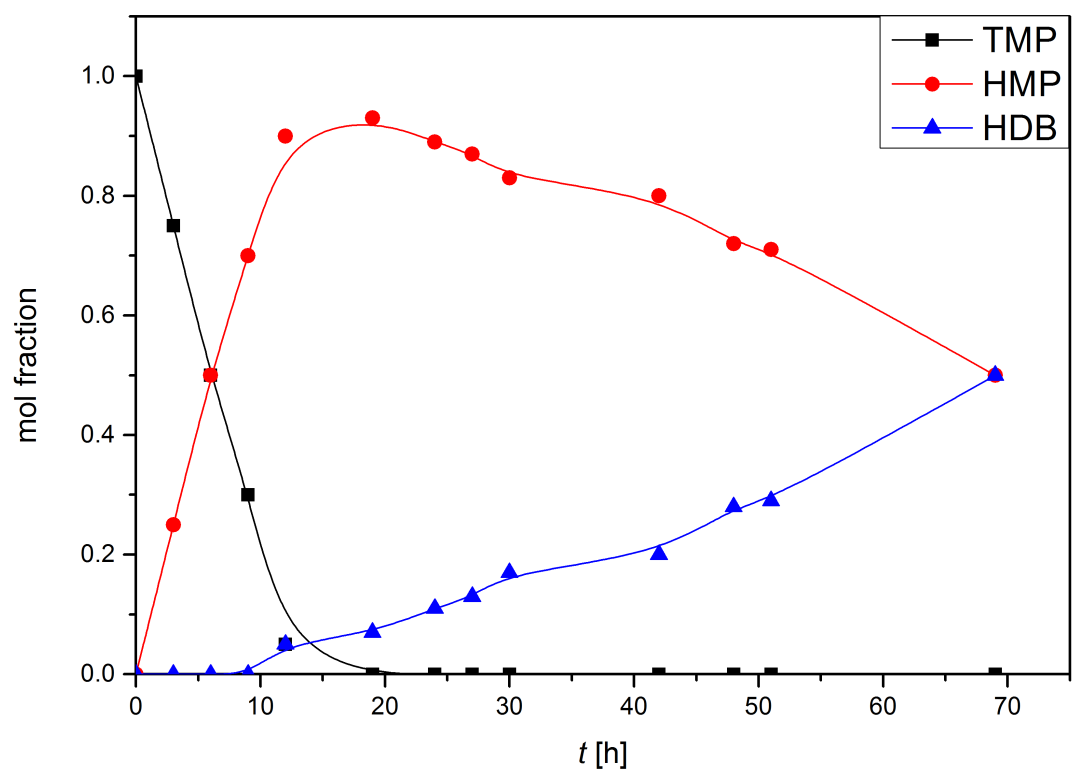

Figure 4.22 concentration profile over time of the conversion of TMP to HMP and subsequently to HDB catalyzed by $\mathbf{1}$.

conversion after 70 hours. Again, a slow but clean conversion was preferred over a rapid reaction with more unwanted side products.

When the reaction was carried out under inert conditions, the formation of a substoichiometric amount of HMP with repsect to the catalyst was observed. HDB was not observed.

\section{EPR spectroscopy}

The recorded spectra show similarity to the previously recorded ones for TMP and MDP. There is a half field signal at $153 \mathrm{mT}$ with a g-value of 4.40 and a main signal at $313 \mathrm{mT}$ with a $g_{\perp}$-value of $2.13\left(\mathrm{~A}=0.0293 \mathrm{~cm}^{-1}\right)$ and a $\mathrm{g}_{\|}$value of $2.08\left(\mathrm{~A}=0.0040 \mathrm{~cm}^{-1}\right)$. The spectrum measured at $6 \mathrm{~K}$ was difficult to interpret due its weak intensity. The simulation revealed a $\mathrm{g}_{\|}$value of 2.19 and an A-value of $0.0082 \mathrm{~cm}^{-1}$. The $\mathrm{g}_{\perp}$-value was found to be 2.05 with an A-Value of $0.0015 \mathrm{~cm}^{-1}$. Although very weak in intensitiy, the presence of a signal at $6 \mathrm{~K}$ shows the presence of a mixed valent copper(II)copper(I) species as observed before.

\section{Origin of the OH-group}

To determine the origin of the hydroxy-function introduced at the 4-methyl group of the phenol, an experiment with ${ }^{18} \mathrm{O}$ labeled water was carried out in dried solvents under nitrogen atmosphere. After workup, the residue was submitted to GC-MS analysis. It was found that the residue contained a mixture of unreacted TMP, mainly HMP and also small amounts of HDB. HMP and HDB both have been found to incorporate the ${ }^{18} \mathrm{O}$-isotope. When the ${ }^{18} \mathrm{O}$ labeled $\mathrm{HDB}$ was dissolved in a mixture of MeCN and $\mathrm{H}_{2}^{16} \mathrm{O}$, 

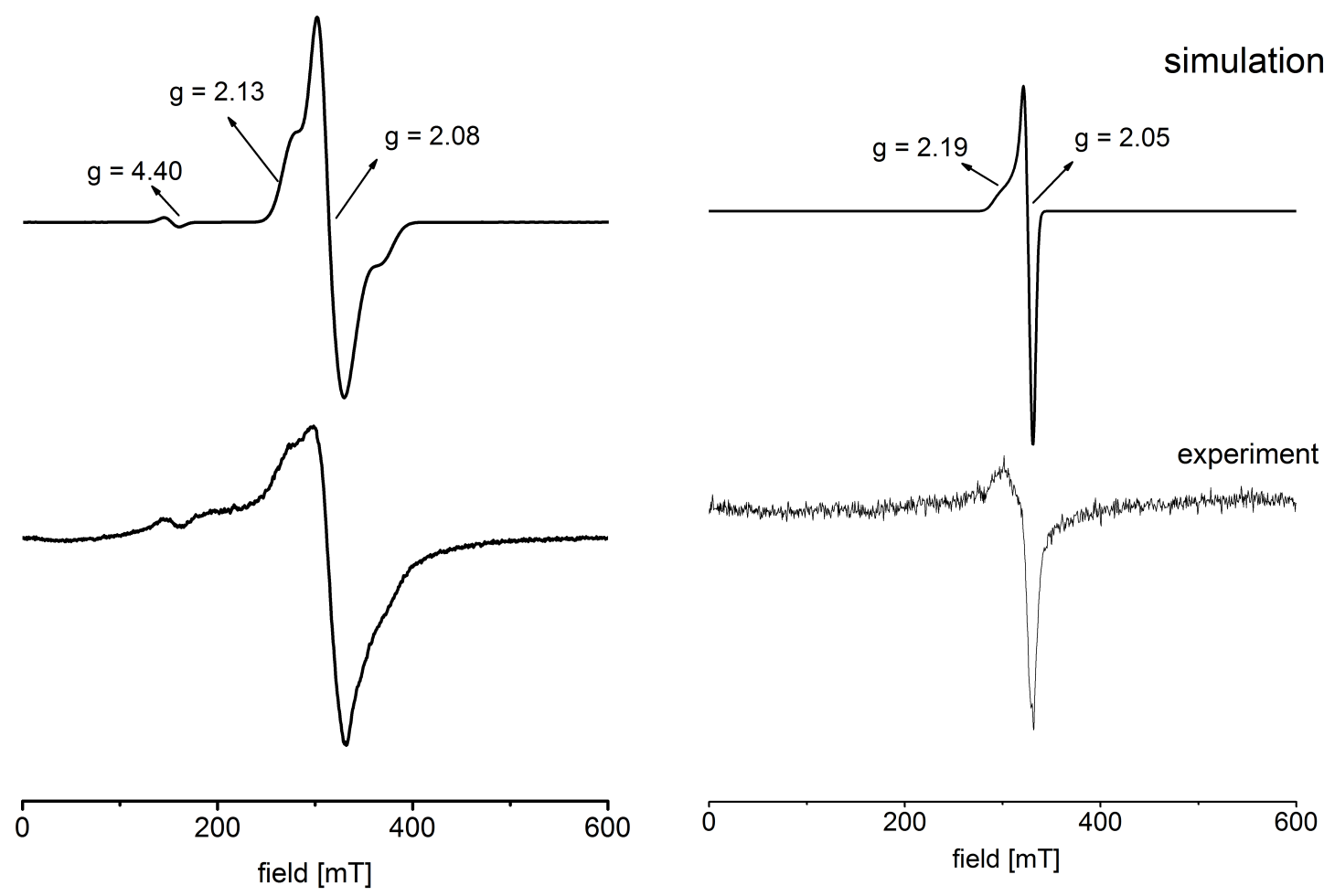

Figure 4.23 a) EPR spectrum of a solution of 1 and TMP in MeCN/acetone/water after stirring over night in a glovebox recorded at $170 \mathrm{~K}$ (simulation parameters: nucleus = $\mathrm{Cu}, \mathrm{Cu}$, total $\mathrm{spin}=1$, linewidth $=8 \mathrm{mT}$, frequency $=9.429 \mathrm{GHz}, \mathrm{g}_{\|}=2.13, \mathrm{~g}_{\perp}=2.08$, $\mathrm{A}_{\|}=0.0140 \mathrm{~cm}^{-1}, \mathrm{~A}_{\perp}=0.0016 \mathrm{~cm}^{-1}$, gStrain $\left.=[0.1,0.1,0.05]\right)$. b)EPR spectrum of the same solution at $6 \mathrm{~K}$ (simulation parameters: nucleus $=\mathrm{Cu}$, total spin $=1 / 2, \mathrm{~T}=6$ $\mathrm{K}$, frequency $=9.368 \mathrm{GHz}$, linewidth $=8 \mathrm{mT}, \mathrm{g}_{\|}=2.19, \mathrm{~g}_{\perp}=2.05, \mathrm{~A}_{\|}=0.0016 \mathrm{~cm}^{-1}$, $\mathrm{A}_{\perp}=0.0033 \mathrm{~cm}^{-1}$, gStrain $\left.=[0.1,0.05,0.05]\right)$.

the labeling vanished due to the typical water exhcange reaction of aldehydes via a hydrated intermediate.

A second experiment where the reaction was carried out with unlabeled water under an ${ }^{18} \mathrm{O}_{2}$ atmosphere showed no incorporation of the heavier isotope. Under the latter conditions, the labeling of HDB is not a reliable indicator as aldehyde functional groups undergo rapid oxygen exchange with surrounding water. Nevertheless, the oxygen seems to originate from the surrounding water in the solvent mixture.

\section{Binding constants}

Binding constants for all compounds were determined using a high affinity binding approach. $\stackrel{186}{ }$ For this purpose several UV/Vis experiments monitoring the LMCT band at the corresponding wavelength were carried out with a constant amount of $\mathbf{1}$ and increasing amount of the phenolic substrate until saturation was reached.

The absorption maxima of the LMCT bands were determined in MeCN as a common solvent. This was chosen to provide a comparable basis for the data. Furthermore, it 
was not possible to determine the binding constant in a solution containing methanol or water, most likely because they are competing with the phenol for the binding site (Figure 4.24).

The adduct with TMP shows an absorption maximum at $490 \mathrm{~nm}$, with HDB at 482 and

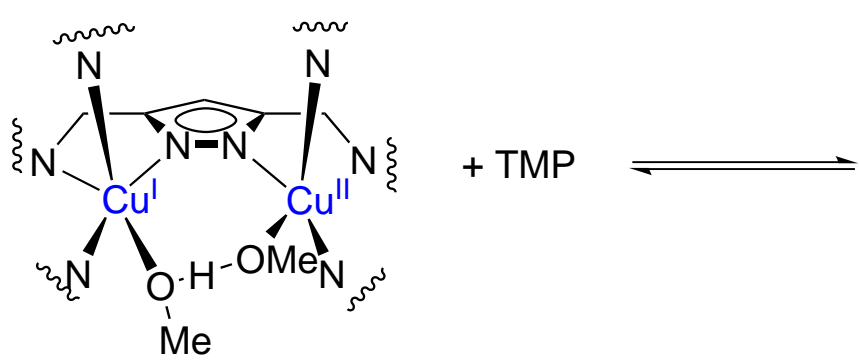

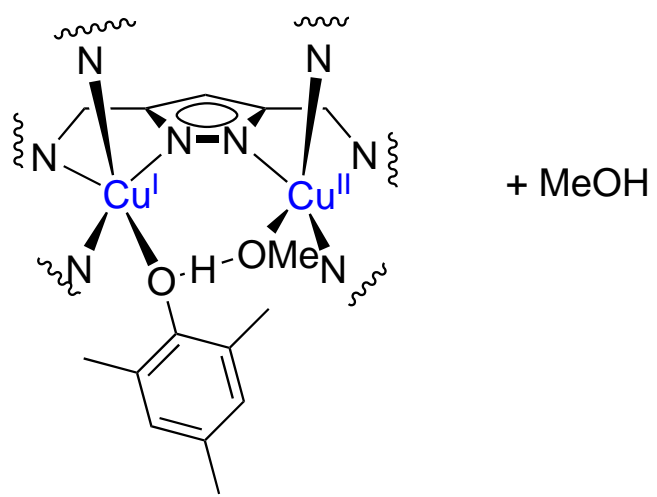

Figure 4.24 Binding equilibrium between TMP and MeOH. An Excess MeOH shifts the equilibrium towards a methoxide bound to the complex.

with HMP and MDP at $479 \mathrm{~nm}$, as can be seen in Figure 4.25. The LMCT band with the

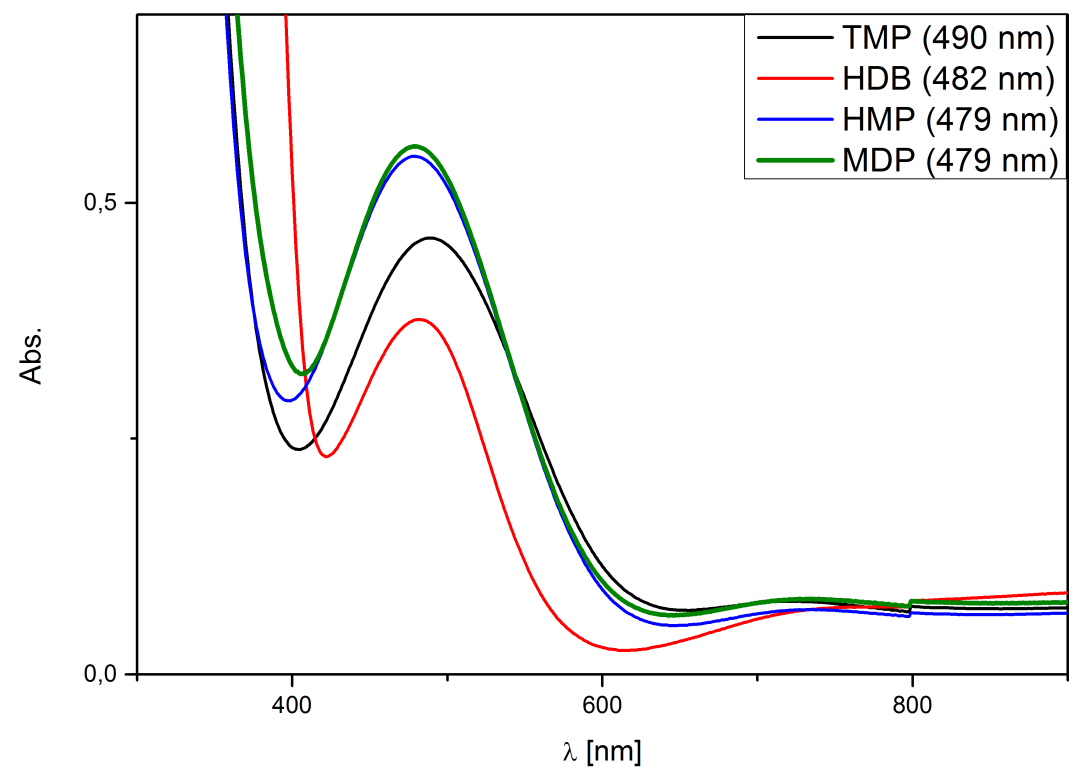

Figure 4.25 UV/Vis spectra of adducts of $\mathbf{1}$ and the phenols used in MeCN. $c(\mathbf{1})=$ $5 \cdot 10^{-4} \mathrm{M}, c($ phenol $)=7.5 \cdot 10^{-3} \mathrm{M}$. black) TMP, red) HDB, blue) HMP, green) MDP. Absorption maxima are mentioned in brackets.

highest intensity is that of the adduct of MDP and just slightly weaker is that of HMP. Significantly lower intensity is observed for the LMCT band of the TMP adduct and even lower that of HDB. The intensity of the bands correlates with the donating strength of each phenolate. ${ }^{187}$. The more electron density available for the charge transfer, the the higher the probability of the transition, which is reflected by a higher intensity of the 
band. The lowest intensity is observed for HDB. With its electron withdrawing formyl function (-M effect), it exhibits the lowest intensity. The adduct with TMP is higher in intensity as the methyl group in para-position acts as an electron donating group with a $+\mathrm{I}$ effect. Higher in intensity are HMP and MDP adducts. Furthermore, a hypsochromic shift can be observed with increasing electron withdrawing capability of the substituent in para position. To determine the binding constants, a fixed concentration of $\mathbf{1}$ was used and in each experiment, an increased amount of phenol was added and the reaction followed over time, until a maximum absorption was reached. The difference in maximum absorption $(\Delta \mathrm{Abs})$ compared to the spectrum without phenol was plotted against the phenol concentration and fitted. ${ }^{186}$ The results are listed in Table 4.2. The obtained results don't follow an appearant rule. The binding constants of MDP and HMP were expected to give very similar results due to their similar structure, that is also reflected in almost identical UV/Vis absorption spectra for their copper(II)-phenolate adducts. It is also noteworthy that HDB, although the aldehyde has an electron withdrawing effect, exhibits by far the largest binding constant.

Table 4.2 Phenol and corresponding binding constant to $\mathbf{1}$.

\begin{tabular}{lc} 
substrate & $\mathrm{K}_{\mathrm{B}} \mathrm{M}^{-1}$ \\
\hline \hline TMP & 614 \\
\hline MDP & 288 \\
\hline HMP & 1100 \\
\hline HDB & 1923
\end{tabular}

\section{Molecular structure of a HDB adduct}

By layering a solution of HDB and $\mathbf{1}$ in acetone with diethyl ether, it was possible to obtain a single crystal suitable for x-ray crystallography (3) (Figure 4.27). In this structure, the deprotonated phenol is bound to one of the copper(II) ions and serves as a hydrogen bond acceptor towards a methanol molecule coordinated to the second copper. The donating properties of the phenolate are known to depend on the $\mathrm{Cu}-\mathrm{O}-\mathrm{C}$ angle, which is reflected in the corresponding $\mathrm{C}-\mathrm{O}$ stretching frequency. In terms of electronics, the angle also reflects the amount of s-orbital character of the $\mathrm{Cu}-\mathrm{O}$ bond. ${ }^{188}$ Moreover, the ring orientation of the phenolate determines whether the in-plane or the out-of-plane $\pi$-orbital (with respect to the aromatic ring) of the oxygen atom is involved in the bond. Based on the findings of SOLOMON and coworkers, the energy of the LMCT changes, depending on the orbital involved. 189

The $\mathrm{C}-\mathrm{O}-\mathrm{Cu}$ angle in the molecular structure of $\mathbf{3}$ is $113.5^{\circ}$. This is just slightly below the observed angles for mononuclear copper phenolate complexes of $117-122^{\circ}$. 189]

The copper-copper distance in $\mathbf{3}$ measures $4.37 \AA$ and is therefore marginally elongated compared to $4.31 \AA$ in $\mathbf{1}$. The copper-nitrogen bond lengths are essentially the same for 


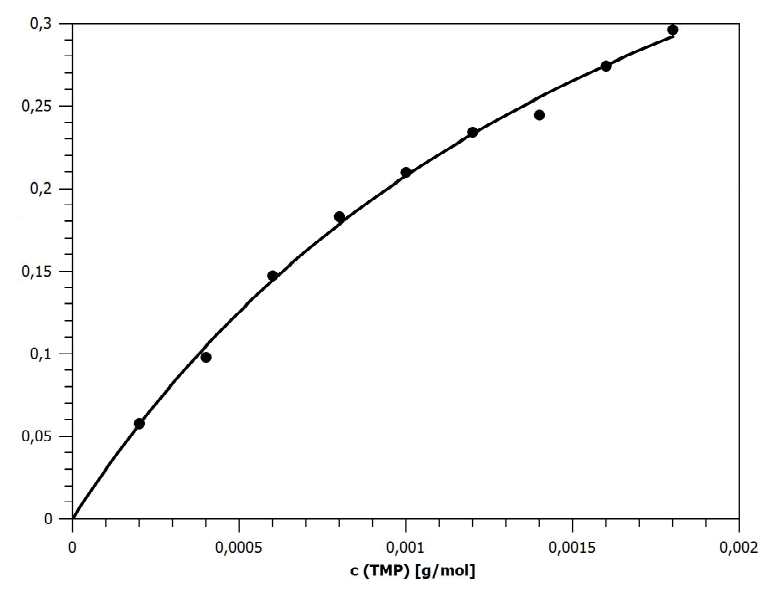

(a) $\triangle$ Abs plotted against the TMP concentration. $c(\mathbf{1})=2 \cdot 10^{-4} \mathrm{M}$ and high affiinity binding fit. The binding constant was determined to be $614 \mathrm{M}^{-1}$

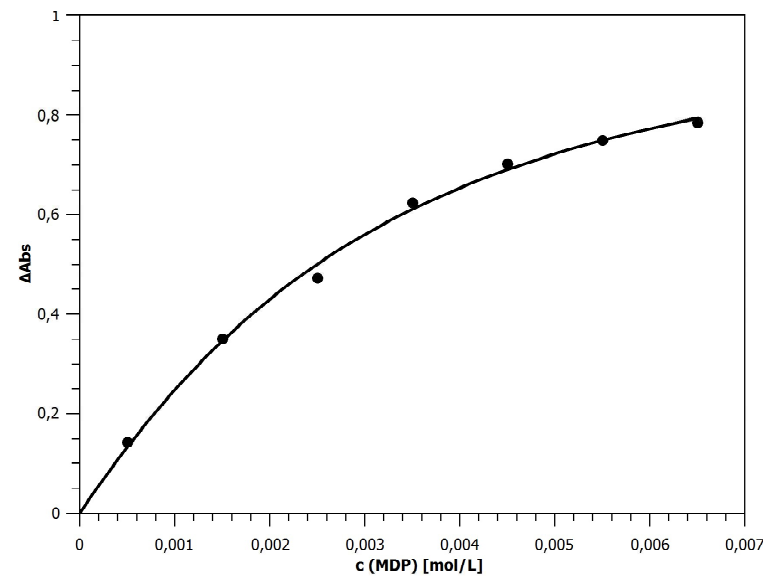

(c) $\triangle$ Abs plotted against the MDP concentration. $c(\mathbf{1})=5 \cdot 10^{-4} \mathrm{M}$ and high affinity binding fit. The binding constant was determined to be $288 \mathrm{M}^{-1}$

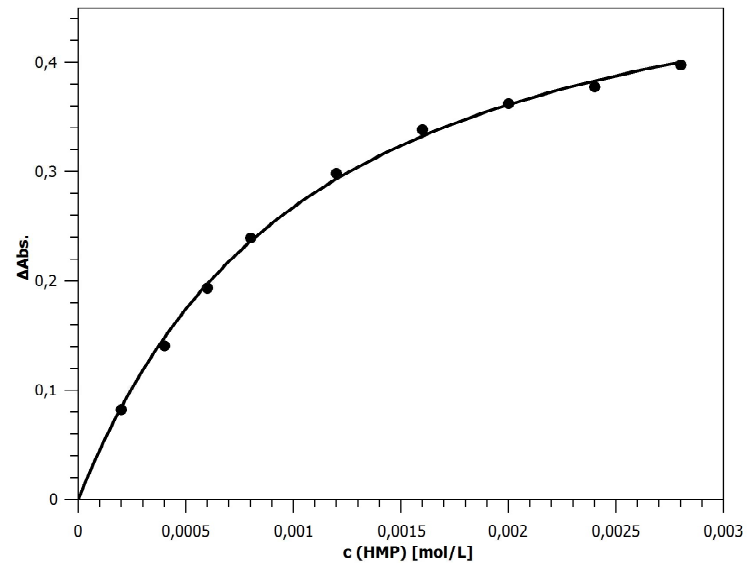

(b) $\triangle$ Abs plotted against the HMP concentration. $c(\mathbf{1})=2 \cdot 10^{-4} \mathrm{M}$ and high affinity binding fit. The binding constant was determined to be $1100 \mathrm{M}^{-1}$

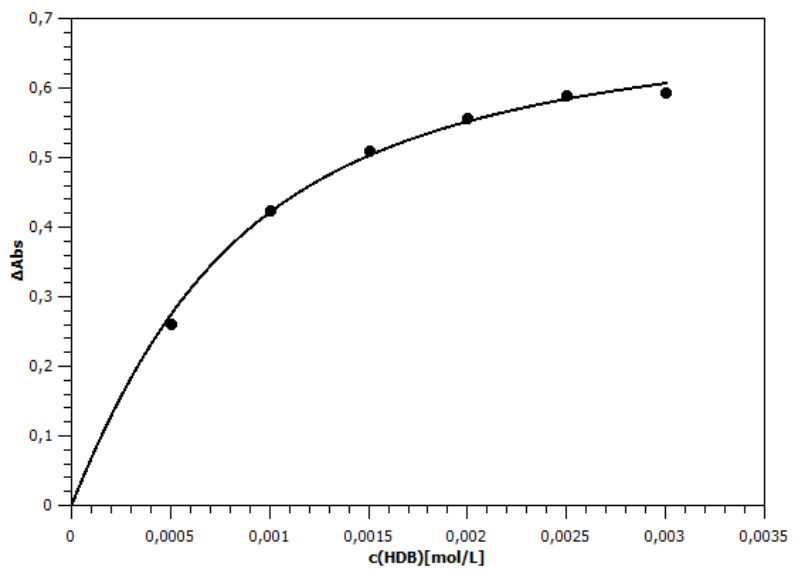

(d) $\triangle$ Abs plotted against the HDB concentration. $c(\mathbf{1})=2 \cdot 10^{-4} \mathrm{M}$ and high affinity binding fit. The binding constant was determined to be $1923 \mathrm{M}^{-1}$

Figure 4.26 High affinity binding plots to detemerine the binding constant of the different phenols to 1 .

both copper ions.

The molecular structure provides a good illustration for the binding mode of the phenolate. Combined with the obtained resonance Raman data, the binding modes of other phenols can be determined.

\section{Resonance Raman Spectroscopy}

Resonance Raman spectra of the adducts were recorded in MeCN at $77 \mathrm{~K}$ Figure 4.28). The $\mathrm{C}-\mathrm{O}$ stretching frequency is known to depend on the $\mathrm{Cu}-\mathrm{O}-\mathrm{C}$ angle and is indicative for the s-orbital character involved in the electron donation from the phenolate 


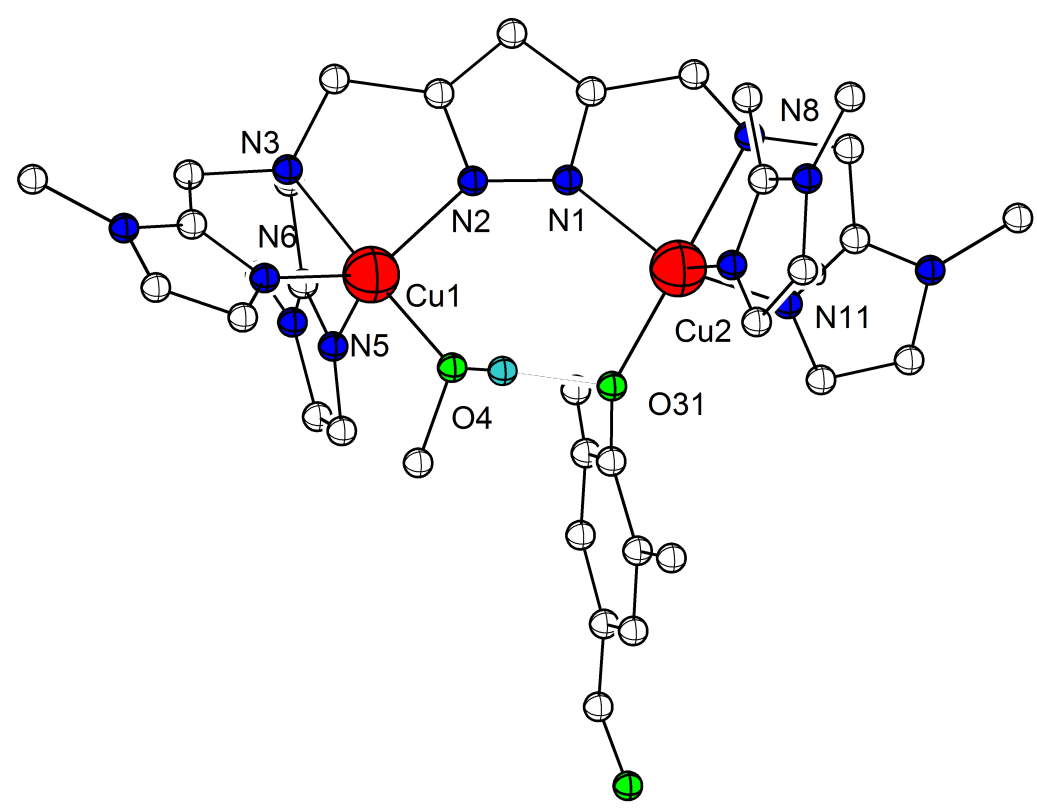

Figure 4.27 Molecular structure of HDB adduct 3, together with a partial labeling scheme; thermal displacement ellipsoids are drawn at $50 \%$ probability.

oxygen to the metal. $\left[\frac{157 / 163}{}\right.$ All spectra look very similar and therefore, it was assumed that all phenols bind in a similar manner.

The $\mathrm{C}-\mathrm{O}$ stretching frequency $\left(\nu_{7 a}\right)$ for the TMP adduct was assigned to $1252 \mathrm{~cm}^{-1}$ and the $\mathrm{C}_{\text {ortho }}-\mathrm{C}_{\text {meta }} \nu_{8 a}$ assigned to $1611 \mathrm{~cm}^{-1}$ by comparison with the spectrum of the adduct of the deuterated TMP- $\mathrm{d}^{9}$ shown earlier in this chapter (Figure 4.3). Based on the assignment of the $\mathrm{C}_{\text {ortho }}-\mathrm{C}_{\text {meta }}$ frequency of the TMP adduct to $1611 \mathrm{~cm}^{-1}$, for HMP the band at $1610 \mathrm{~cm}^{-1}$, for MDP the band at $1611 \mathrm{~cm}^{-1}$ and for HDB the band at $1600 \mathrm{~cm}^{-1}$ were assigned to the $\mathrm{C}_{\text {ortho }}-\mathrm{C}_{\text {meta }}$ stretch. The band at $1669 \mathrm{~cm}^{-1}$ for the $\mathrm{HDB}$ adduct corresponds to the $\mathrm{C}=\mathrm{O}$ stretch of the aromatic aldehyde.

The $\mathrm{C}-\mathrm{O}$ stretching frequency for the TMP adduct is found at $1252 \mathrm{~cm}^{-1}$. For HMP and MDP the only band close to that of TMP is found at $1230 \mathrm{~cm}^{-1}$ and $1248 \mathrm{~cm}^{-1}$ respectively. For HDB the band at $1278 \mathrm{~cm}^{-1}$ was assigned to the $\mathrm{C}-\mathrm{O}$ stretch.

All phenols exhibit similar UV/Vis and resonance raman spectra. Therefore, it can be assumed, that they also exhibit very similar binding motifs and $\mathrm{Cu}-\mathrm{O}-\mathrm{C}$-angles, when bound to a copper ion of $\mathbf{1}$. 

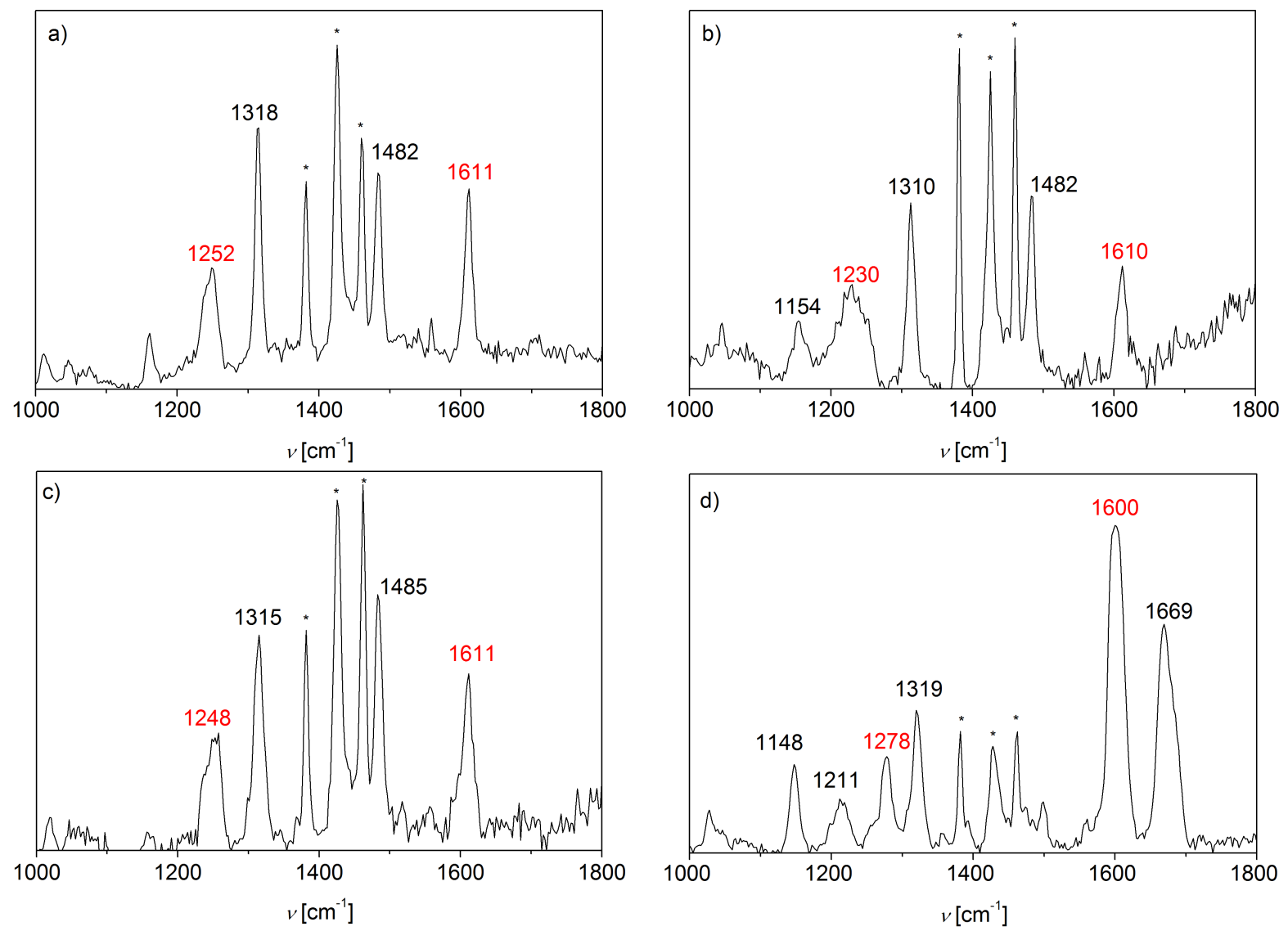

Figure 4.28 a) rR spectrum of the TMP adduct; b) rR spectrum of the HMP adduct; c) $r R$ spectrum of the MDP adduct; d) rR spectrum of the HDB adduct. Solvent signals are denoted with an asterisk. Assigned bands are marked red.

\subsubsection{Direct synthesis of 3,5-dimethyl-4-hydroxy benzaldehyde}

In the previous chapter, a two step mechanism for synthesis of HDB with a catlytic amount of base (stemming from the catalyst) was desribed, this section will focus on a faster, direct way to synthesize HDB by using of two equivalents of base. Several bases have been screened and it was found that either sodium-2,4,6-trimethyl phenolate or strong amine bases are best suited for the reaction. Other alkoxides such as $\mathrm{KO}^{t} \mathrm{Bu}$ and weak amine bases are not suitable for this reaction. In general, alkoxides are disfavoured and the $\mathrm{pK}_{\mathrm{A}}$-value of the base has to exceed that of the TMP hydroxy function $\left(\mathrm{p} K_{\mathrm{a}}(\mathrm{TMP})\right.$ $\left.=10.88^{[190}\right)$.

For the reaction to proceed in the mentioned manner, 1, TMP and one equivalent of DBU (with respect to the catalyst, $\mathrm{p} K_{\mathrm{a}}=13.5$ in water ${ }^{191} ; \mathrm{p} K_{\mathrm{a}}=24.1$ in $\mathrm{MeCN}^{192}$ ) were dissolved in MeCN. With the endogenous methoxide present in the catalyst and the additional DBU, a total of two equivalents of base are present in the reaction mixture. After stirring over night, the solution was filtered through silica and all volatile material was removed under reduced pressure. The main product was found to be HDB in good 
yield and purity.

Experiments with an increased amount of DBU revealed an overall beneficial effect on

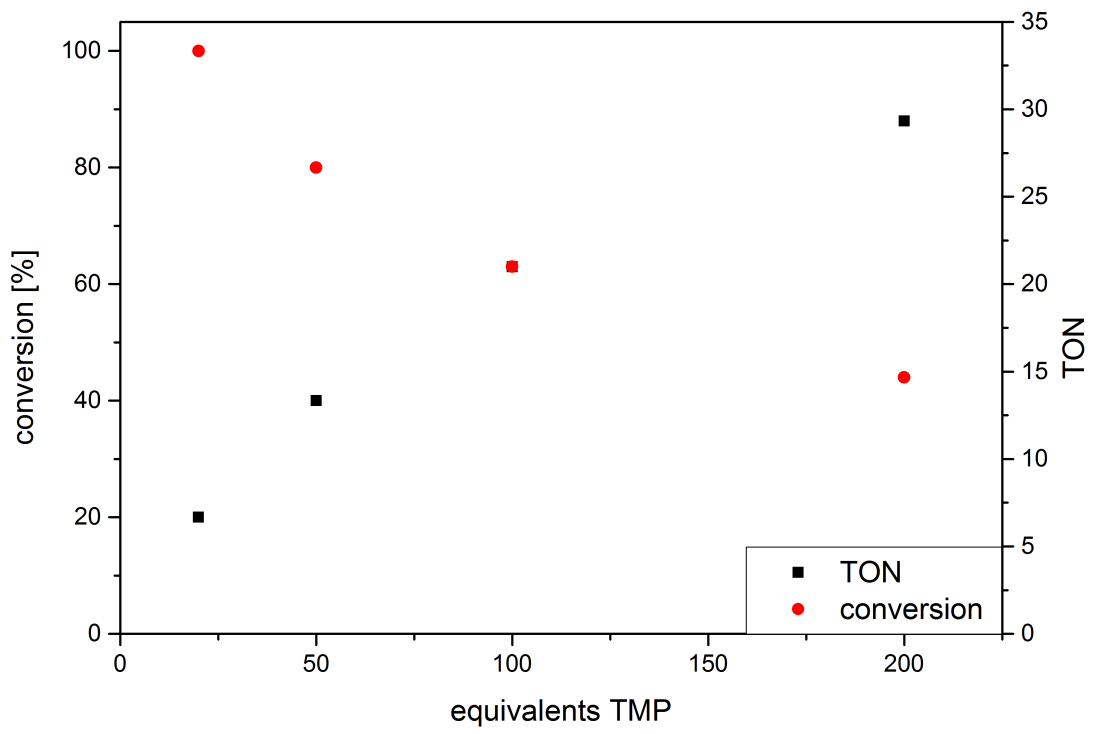

Figure 4.29 TON plotted against equivalents of TMP used.

the reaction rate as well as the purity of the product. In fact, a clean and quantitative conversion could be achieved. For this reason, it was decided to work with equimolar amounts of TMP and DBU. The ideal catalyst concentration was found to be about 5 mol\% (Figure 4.29). Total conversion was achieved and a yield of $95 \%$ clean product was possible. As can be seen in Figure 4.29, a complete conversion is reached for 20 equivalents of substrate, while 50 equivalents (2mol\% catalyst loading) result in a TON of 40 which corresponds to a conversion of $80 \%$. Further decrease of catalyst concentration further lowers the conversion rate. This is most likely due to catalyst decomposition. Regarding the concentration of the reactants, it was found that a higher concentration is beneficial for the reaction rate (Figure 4.30). While the reaction proceeds faster at a $2 \mathrm{~mm}$ concentration in the beginning, the same conversion as the $1 \mathrm{~mm}$ solution is reached after two hours. When the concentration is lowered to $0.5 \mathrm{mM}$ the reaction progresses slower and about $70 \%$ conversion is reached after two hours.

Upon addition of DBU to the deep red reaction mixture, it turns intensly purple. UV/Vis spectroscopy reveals a shift of the LMCT band from $495 \mathrm{~nm}$ to $522 \mathrm{~nm}$ along with an increase in its intesity, indicating a higher probability of the electronic transition from the phenolate to the copper(II) ion (Figure 4.31). It is assumed that the base is abstracting a proton from the para- $\mathrm{CH}_{3}$ group of the bound phenolate and in consequence increasing its charge, which is resultig in a higher readiness to donate electrons to the metal center. Additionally, a small shoulder is rising at $580 \mathrm{~nm}$.

Under anaerobic conditions the LMCT band, the $d$ - $d$ band and the one at $338 \mathrm{~nm}$ decay 


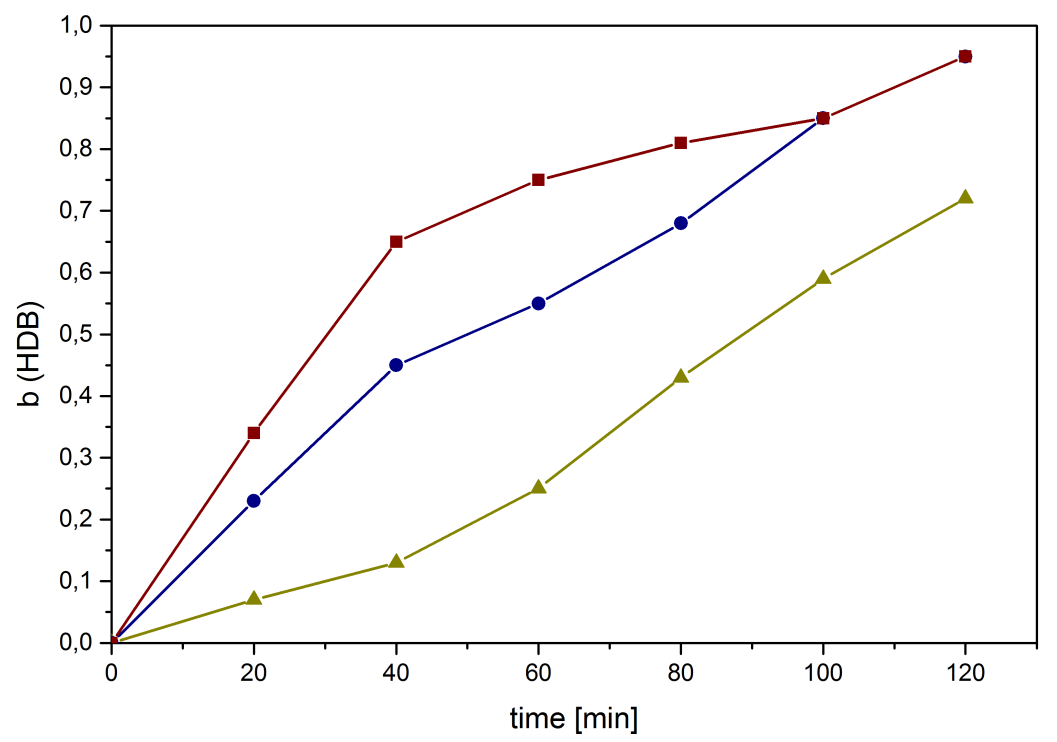

Figure 4.30 mol fraction (HDB) plotted against time at three different concentrations. $\mathrm{n}(\mathbf{1})=16 \mu \mathrm{mol}, \mathrm{n}(\mathrm{TMP})=\mathrm{n}(\mathrm{DBU})=320 \mu \mathrm{mol}$. red) $2 \mathrm{~mm}$, blue) $1 \mathrm{~mm}$, yellow $)$ $0.5 \mathrm{~mm}$.

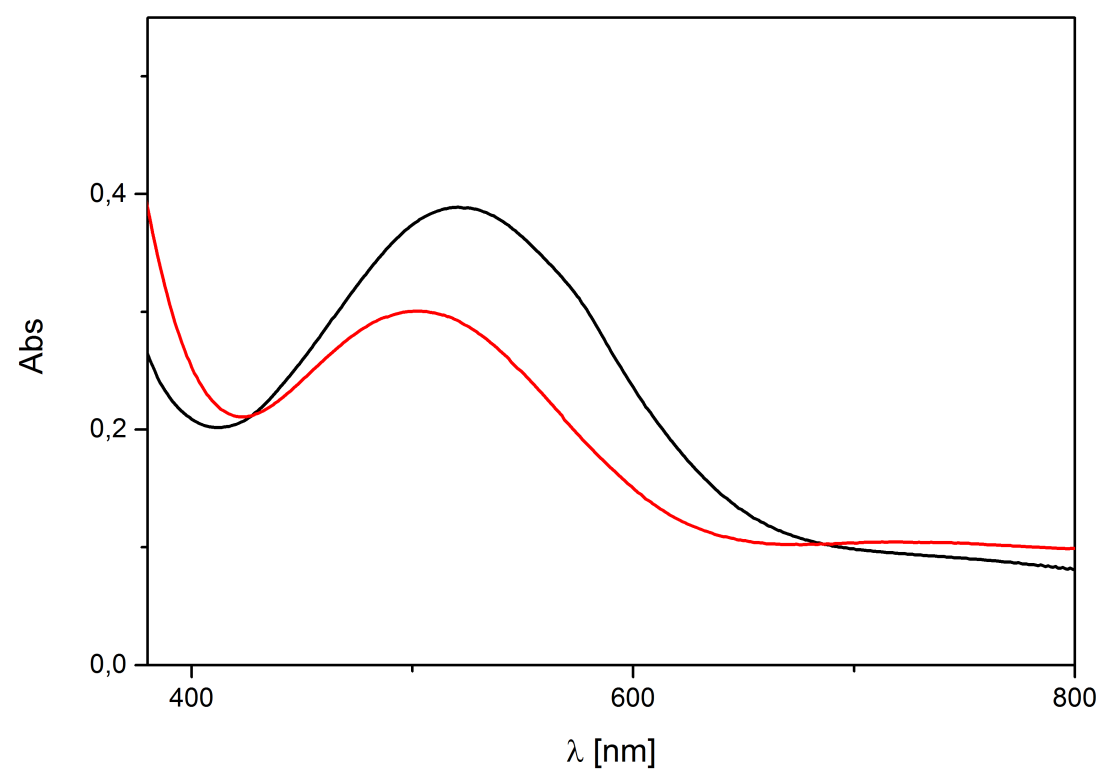

Figure 4.31 Red line) absorption of $\mathbf{1}$ and TMP in MeCN $\lambda_{\max }=495 \mathrm{~nm}$. Black line) absorption after addition of DBU $\left(c(\mathbf{1})=2 \cdot 10^{-4} \mathrm{M}, c(\mathrm{TMP})=c(\mathrm{DBU})=10^{-3} \mathrm{M}, \lambda_{\max }\right.$ $=522 \mathrm{~nm}$.

over a period of about 50 minutes and result in a colourless solution. The resulting loss of colour and the disappearace of the $d-d$ band indicate a reduction of the dicopper(II) complex to its dicopper(I) state. Figure 4.32 shows a UV/Vis spectrum of the starting solution under anaerobic and 1 minute after addition of an equimolar amount of DBU. The LMCT band disappeared, while the previously observed shoulder gains in intensity. Another feature rises at $410 \mathrm{~nm}$ which is attributed to the protonated DBU. When the 
colourless solution is exposed to oxygen, the band at $580 \mathrm{~nm}$ gains in intensity as well as the previously decreased band at $338 \mathrm{~nm}$.

While the band at $338 \mathrm{~nm}$ is most likely ligand based, the band at $580 \mathrm{~nm}$ is of unknown

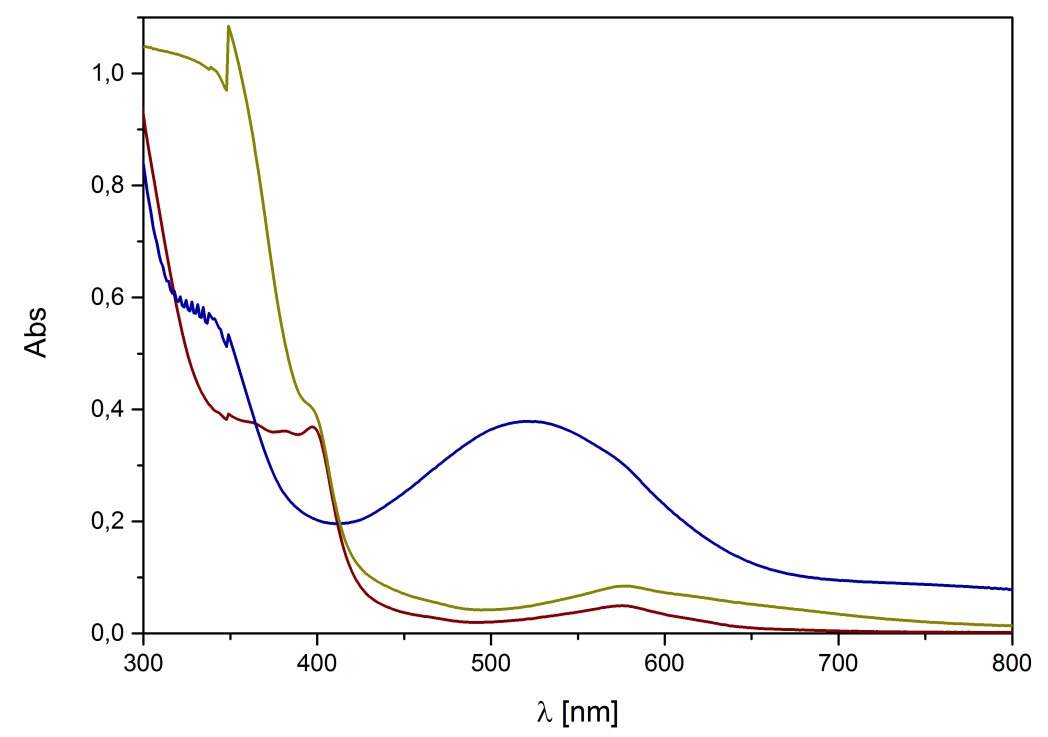

Figure 4.32 UV/Vis spectrum of a solution containing 1, TMP and DBU. blue line) one minute after addition of DBU. red line) after completion of the reaction. yellow line) after opening to the atmosphere.

origin. It is no longer present after the solution was filtered through silica and therefore it originates from the catalyst as it is removed by the filtration. A solution containing $\mathbf{1}$ and DBU does not show this band and therefore its origin does not seem to be a deprotonated species of the complex.

Additionally, it was found that under anaerobic conditions no formation of HDB was observed, when the reaction solution was worked up by filtration through silica, although the complex is reduced, as indicated by the vanishing of the $d-d$ band. 


\section{EPR Spectroscopy}

The oxidation state of the dicopper catalyst after the the solution lost its colour was investigated by means of EPR-spectroscopy. The UV/Vis spectrum of the solution under inert conditions (Figure 4.32) already indicated a reduction of the dicopper(II) complex to its dicopper(I) state. This assumption was confirmed by EPR spectroscopy. A

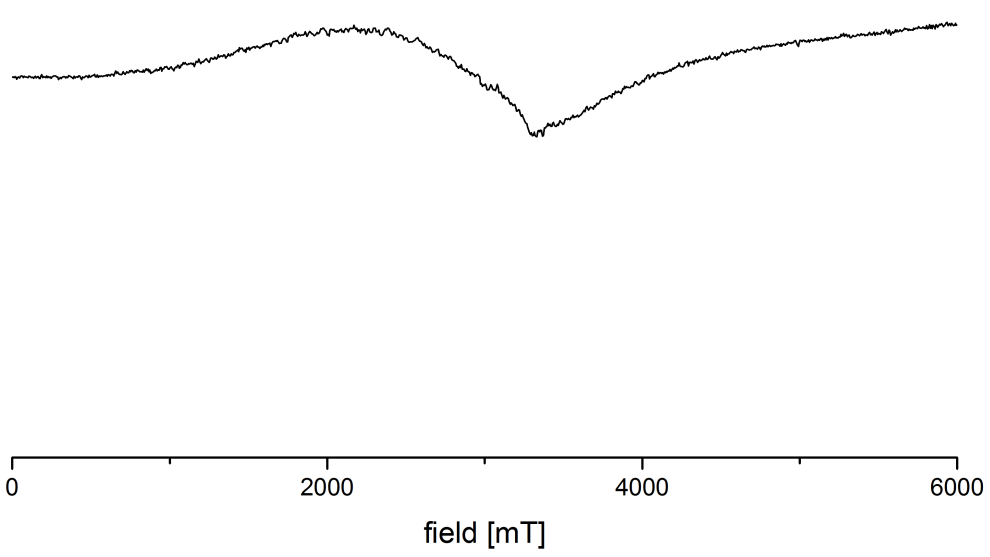

Figure 4.33 EPR spectrum in MeCN at $150 \mathrm{~K}$ recorded after the reaction of $\mathbf{1}$, TMP and DBU.

spectrum recorded at $150 \mathrm{~K}$ in MeCN showed only background signals and no indication of a copper(II) species (Figure 4.33).

\section{Isotope labeling experiment}

Isotope labeling experiments with ${ }^{18} \mathrm{O}$-dioxygen in dry $\mathrm{MeCN}$ revealed a $100 \%$ incorporation of the ${ }^{18} \mathrm{O}$-isotope into the final product.

\section{Oxygen uptake experiment}

An oxygen uptake experiment was performed for the reaction. The results are depicted in Figure 4.34.

The reaction was carried out in a sealed vessel with $2 \mathrm{~mL}$ MeCN solution containing $10 \mu \mathrm{mol}$ of $\mathbf{1}$, TMP and DBU. The reaction was finished after 3 hours and a total amount of $10.2 \mu \mathrm{mol}$ of oxygen was consumed. This results in a stoichiometry of TMP $/ \mathrm{O}_{2}$ of $1 / 1$. From the isotope labeling study it is known, that the incorporated oxygen atom of the product originates from dioxygen. It is further known, that the catalyst is reduced from its initial dicopper(II) state to a dicopper(I) state. Therefore, the complex needs to 


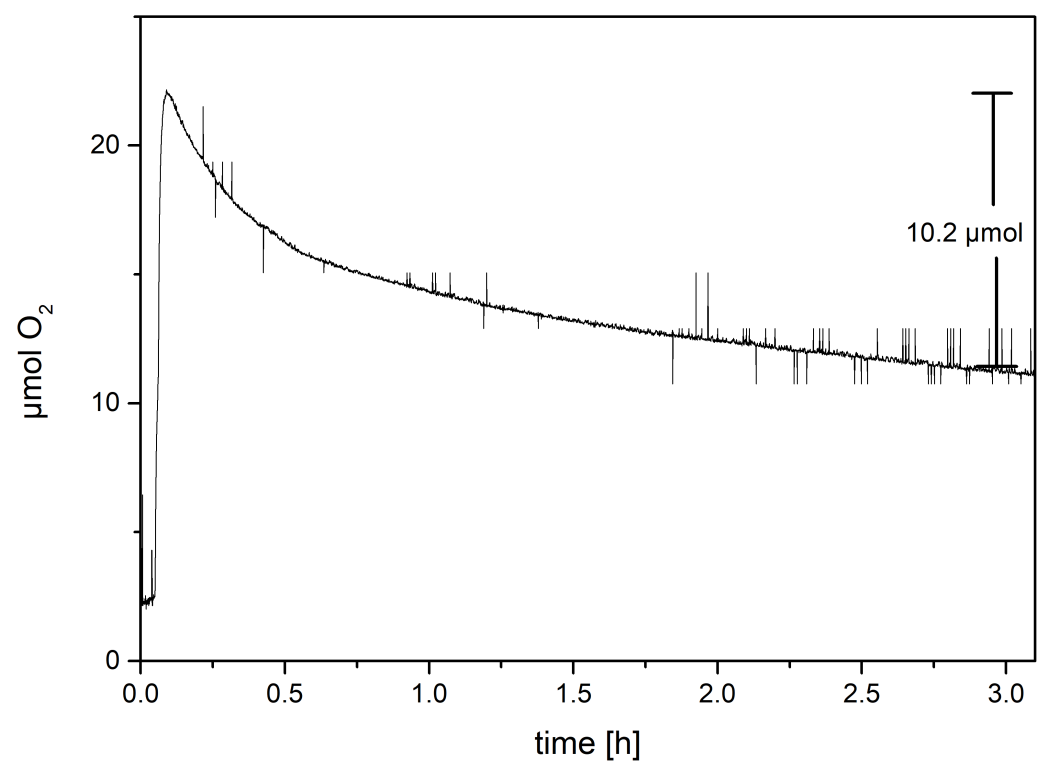

Figure 4.34 Oxygen uptake experiment of a solution containing $10 \mu \mathrm{mol}$ of $\mathbf{1}$, TMP and DBU.

be oxidized by two electrons to return to its initial state. These two electrons can be taken up by the second oxygen atom that is consumed during the reaction. The required protons to form water with the reduced $\mathrm{O}^{2-}$ species are likely to originate from the para- $\mathrm{CH}_{3}$ of TMP. During the cause of the reaction two protons are removed from this position. One of them is taken up by the added $\mathrm{DBU}$, so proton transfer from $\mathrm{DBUH}^{+}$ to the $\mathrm{O}^{2-}$ species would also regenerate the base to undergo another catalytic cycle. In conclusion, one molecule of dioxygen is consumed per catalytic cycle. One atom ends<smiles>Cc1cc(C)c(O)c(C)c1</smiles>

Scheme 4.3 Reaction scheme of the conversion of TMP to HDB mediated by $\mathbf{1}$.

up in the organic product and the other one reoxidizes the complex and is reduced to water Scheme 4.3). This is in contrast to the previous two step reaction to generate HDB where the oxygen stemmed from the solvent, rather than dioxygen. Therefore, a different reaction mechanism must be considered to explain the reactivity at hand. This topic will be discussed further in Chapter 5.3. 


\subsection{Polymerization of 4-bromo-2,6-dimethyl phenol}

The investigated phenols in the previous sections thus far only undergo para- $\mathrm{C}-\mathrm{C}$ coupling or functionalization. In previous work, PROKOFIEVA observed that the bromo substituted 4-bromo-2,6-dimethyl phenol (DMBrP) is catalytically $\mathrm{C}-\mathrm{O}$ coupled by $\mathbf{1}$ in 1,4-position to form poly-(2,6-dimethylphenylene oxide). Prokofieva found that $\mathbf{1}$ catalyzes the nucleophilic substitution of the bromo function by another molecule of DMBrP in MeCN to form a white precipitate. EI mass spectrometry revealed that the compound is a

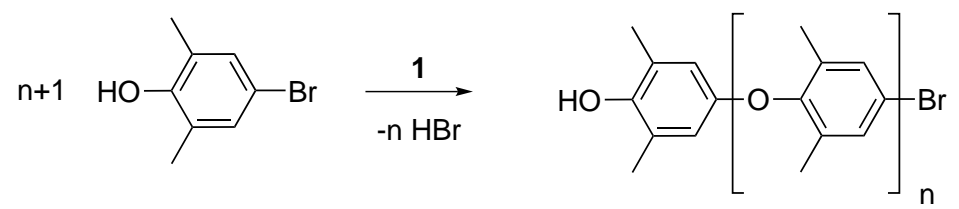

Scheme 4.4 Reaction scheme of the polymerization of 4-bromo-2,6-dimethyl phenol catalyzed by 1 .

polymer of 10-12 monomer units. Additionally, x-ray crystallography revealed a structure, wherein both copper ions were coordinated by a bromide and the pyrazole was found to be protonated, suggesting the release of $\mathrm{HBr}$ during the polymerization.

In this work, the reaction conditions and the work-up of the product was further optimized and the product, as well as the mechanism investigated. It was found that addition of a base such as sodium carbonate significantly increases the chain length of the obtained polymer.

\subsubsection{Investigation of the mechanism}

Upon addition of 10 equivalents DMBrP to a solution of 1 in MeCN, a LMCT band arises at $462 \mathrm{~nm}$ and a feature at $332 \mathrm{~nm}$ decreases within five minutes, as can be seen in Figure 4.35. An isosbestic point for this conversion is observed. The $d-d$ band at around $780 \mathrm{~nm}$ remains unaffected. Compared to TMP, a blue shift of the LMCT band of $33 \mathrm{~nm}$ is observed. The electron withdrawing bromo substituent makes an electronic transition from the phenol to the metal less likely and therefore more energy is needed to excite the transition.

When the reaction is carried out with 100 equivalents of DMBrP, in the same time frame of five minutes, the reaction progresses further. The decrease of the band at $332 \mathrm{~nm}$ is again observed. At the same time a new band arises at $320 \mathrm{~nm}$. A LMCT band at $462 \mathrm{~nm}$ rises within the first minute but quickly decreases again, while a new band at $410 \mathrm{~nm}$ arises (Figure 4.37). When taking a closer look at the second part of the reaction (Figure 4.37 (inset)), after the binding period is complete, there seems to be a clean interconversion between two species as indicated by the isosbestic point. This new 


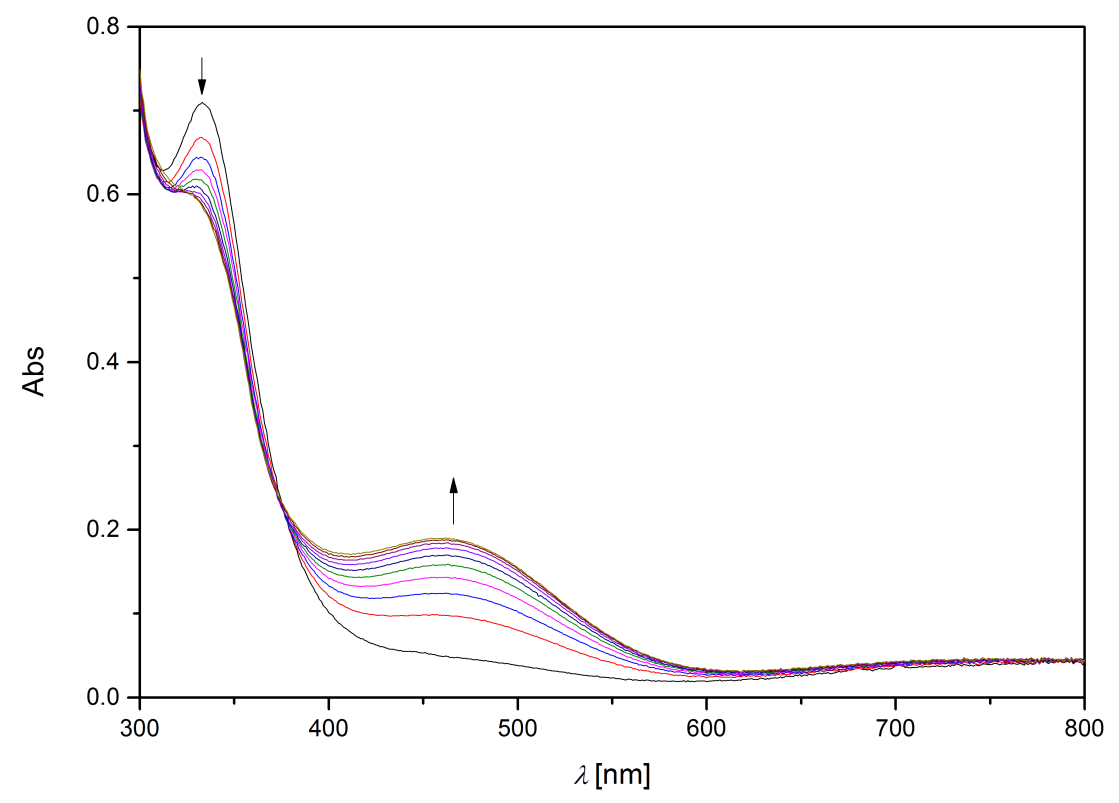

Figure 4.35 UV/Vis spectra of a solution of $1\left(c=5 \cdot 10^{-4} \mathrm{M}\right)$ and 10 equivalents of $\mathrm{DMBrP}$ in MeCN at room temperature; one spectrum every 30 seconds for 5 minutes.

species is most likely the previously observed bromo complex as indicated by the colour and confirmed by ESI-mass spectrometry (Figure 4.36). Without additional base the

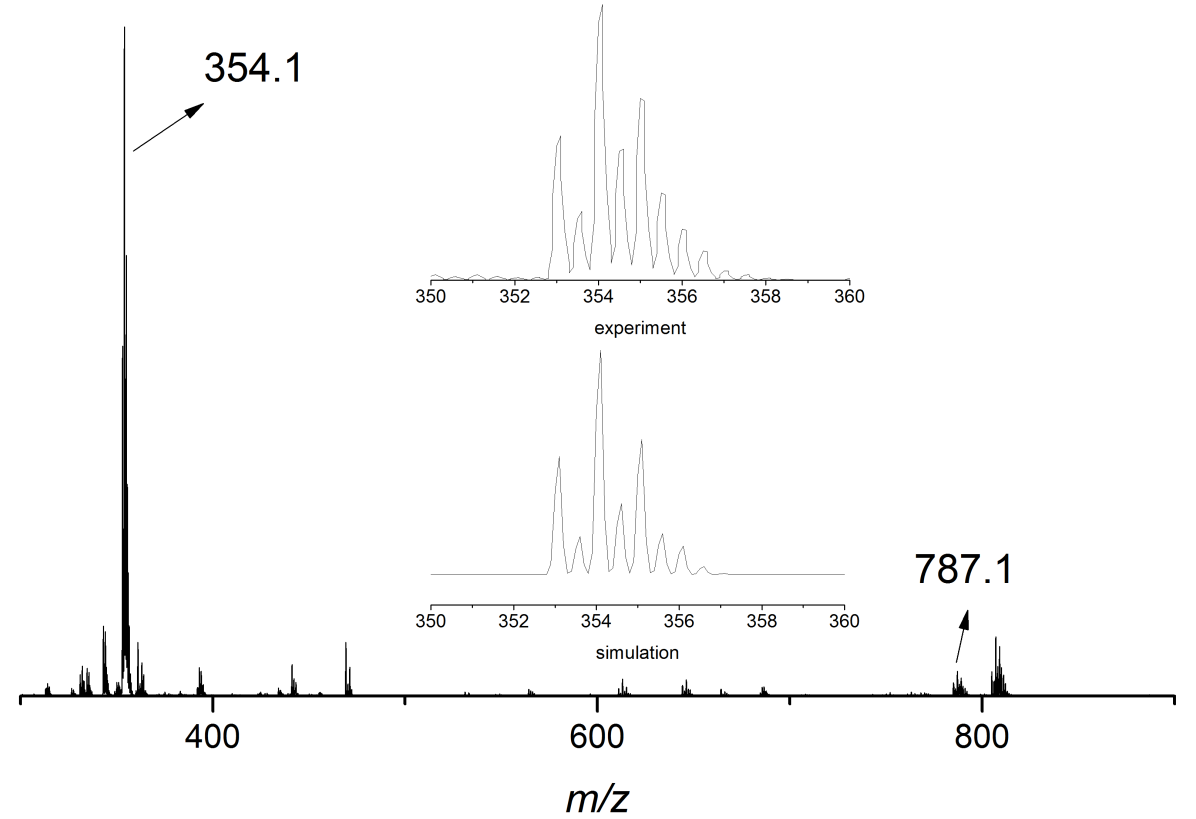

Figure 4.36 Positive ESI-MS spectrum of the blue solution after reaction of 1 with DMBrP. The peak at $m / z=354.1$ was assigned to $\left[\mathbf{L}^{1} \mathrm{Cu}_{2} \mathrm{Br}\right]^{2+}$; the one at $\mathrm{m} / z=$ 787.1 to $\left.\mathbf{L}^{1} \mathrm{Cu}_{2} \mathrm{Br}_{2}\right]^{+}$. The inset shows an enlargement of the peak at $m / z=354.1$ and a simulation of the isotopic pattern for $\left[\mathrm{L}^{1} \mathrm{Cu}_{2} \mathrm{Br}\right]^{2+}$.

yield of polymer, based on the weight of precipitated solid, does not increase with the amount of substrate, which most likely is due to the inactivation of the complex by by 


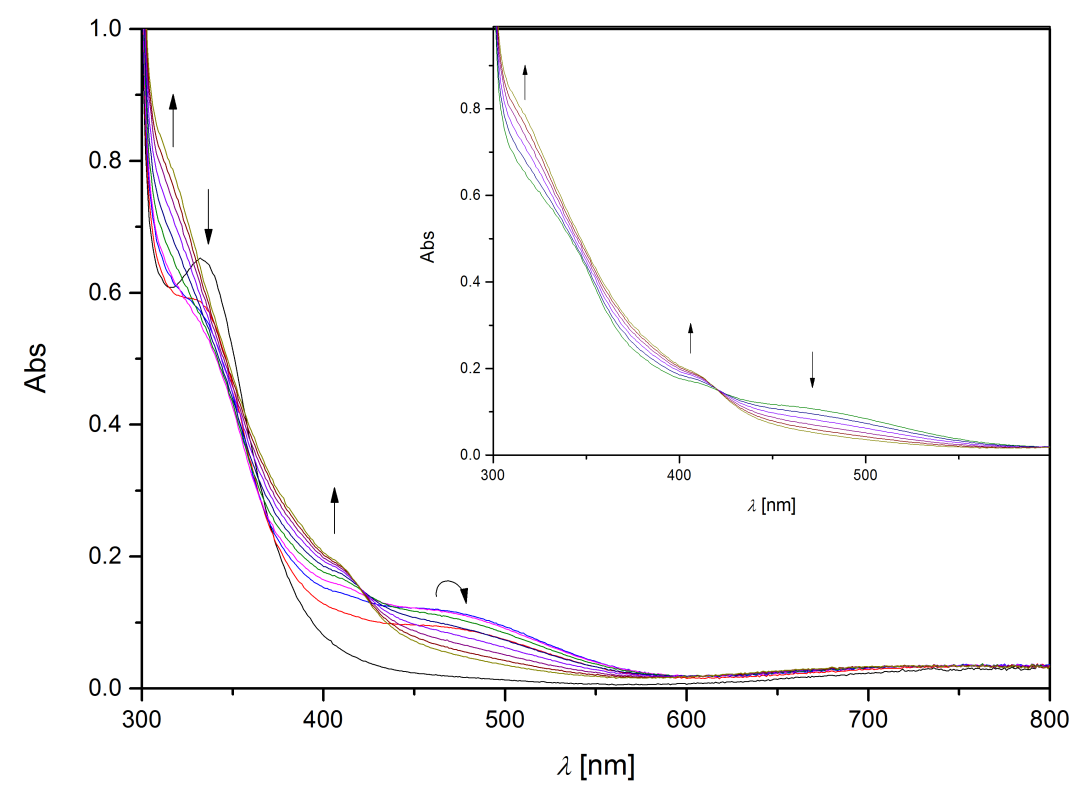

Figure 4.37 UV/Vis spectra of a solution of $1\left(c=5 \cdot 10^{-4} \mathrm{M}\right)$ and 100 equivalents of $\mathrm{DMBrP}$ in $\mathrm{MeCN}$ at room temperature; one spectrum every 30 seconds. Inset: 2.5-10 minutes from 300 to $600 \mathrm{~nm}$.

the formed $\mathrm{HBr}$.

To remove $\mathrm{HBr}$ from the solution, different bases have been tested and $\mathrm{Na}_{2} \mathrm{CO}_{3}$ was

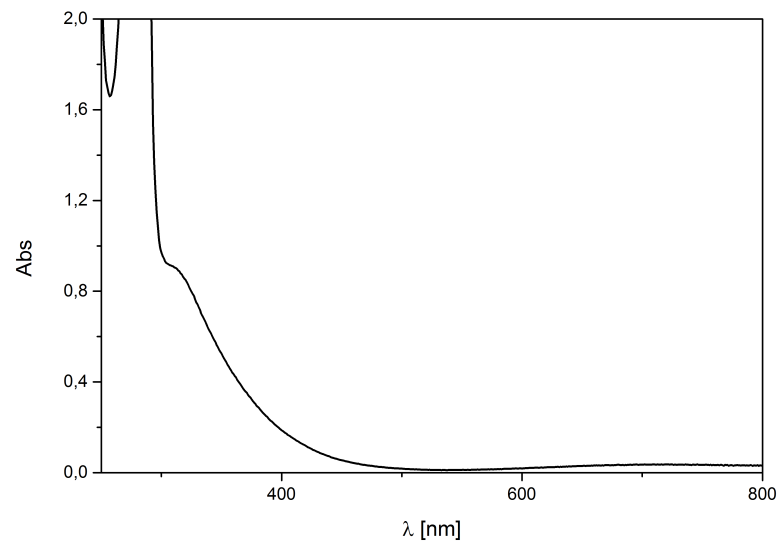

(a) UV/Vis spectrum after the reacion of DM$\mathrm{BrP}$ and $\mathbf{1}$ is completed

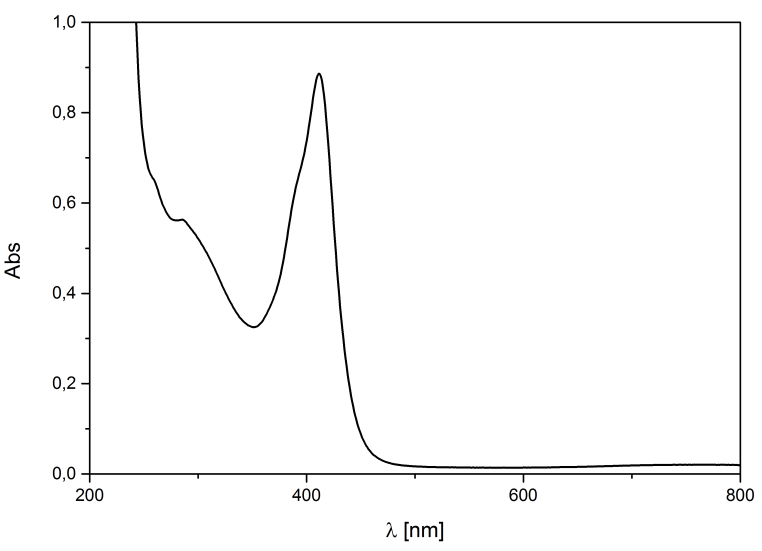

(b) UV/Vis spectrum after the reaction with additional $\mathrm{Na}_{2} \mathrm{CO}_{3}$ is completed

Figure 4.38 UV/Vis spectra after the reaction of DMBRP and 1 under different conditions.

found to be most suitable due to its limited solubility in MeCN, which makes it easy to remove after the reaction, and its low price to keep the overall cost for obtaining the polymer low. The UV/Vis behaviour remains the same for the beginning of the reaction but changes drastically after the reaction is completed (Figure 4.38). In absence of base a band at $310 \mathrm{~nm}$ is visible alongside a weak band at $710 \mathrm{~nm}$ that both correspond to the observed complex $\left[\mathrm{L}^{1} \mathrm{Cu}_{2} \mathrm{Br}_{2}\right]^{+}$. 
In the presence of $\mathrm{Na}_{2} \mathrm{CO}_{3}$ the band at $310 \mathrm{~nm}$ disappears and a shoulder at $260 \mathrm{~nm}$, a new band at $285 \mathrm{~nm}$ and a new intense peak at $412 \mathrm{~nm}$ with a shoulder at $388 \mathrm{~nm}$ appear. While it was not yet possible to identify the nature of these species, ESI-MS revealed the presence of of a bromide and a carbonate adduct. Their possible structures are depicted in Scheme 4.5. The carbonate and the bromide can bind to both copper ions and are probably found in a briding situaion. However, the observed UV/Vis band
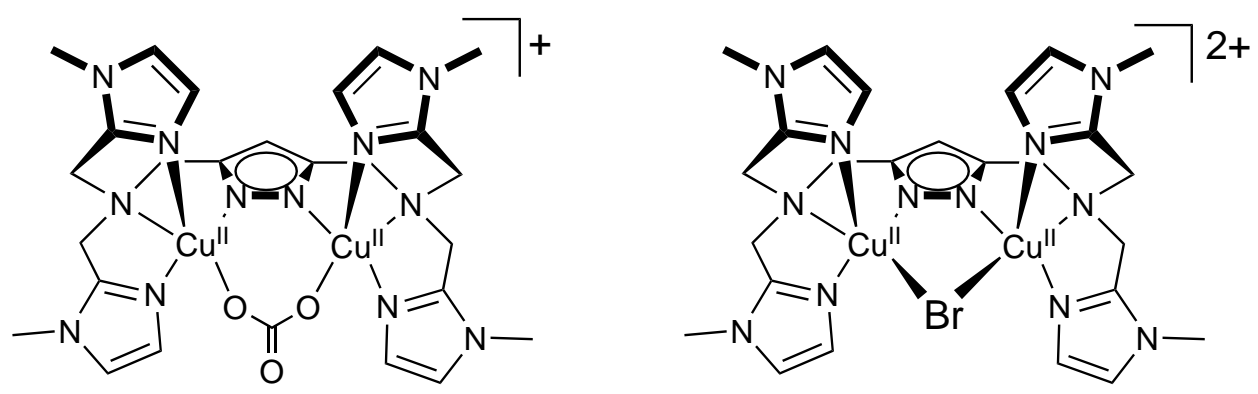

Scheme 4.5 Schematic representations of the expected structures observed by ESI- mass spectrometry.

at $412 \mathrm{~nm}$ does not originate from either of these species, as the preparation of reference spectra with 1 and tetra butyl ammonium bromide ( $\mathrm{TBABr}$ ) and $\mathrm{Na}_{2} \mathrm{CO}_{3}$ as well as tetrabutylammonium carbonate did not show this feature. Its origin remains unknown.

As for the previous substrates the binding constant of DMBrP was determined by means of $\mathrm{UV} / \mathrm{V}$ is spectroscopy. In this case the spectra were recorded at $0^{\circ} \mathrm{C}$ to slow down the reaction. The high affinity binding fit of the difference in absorption for an

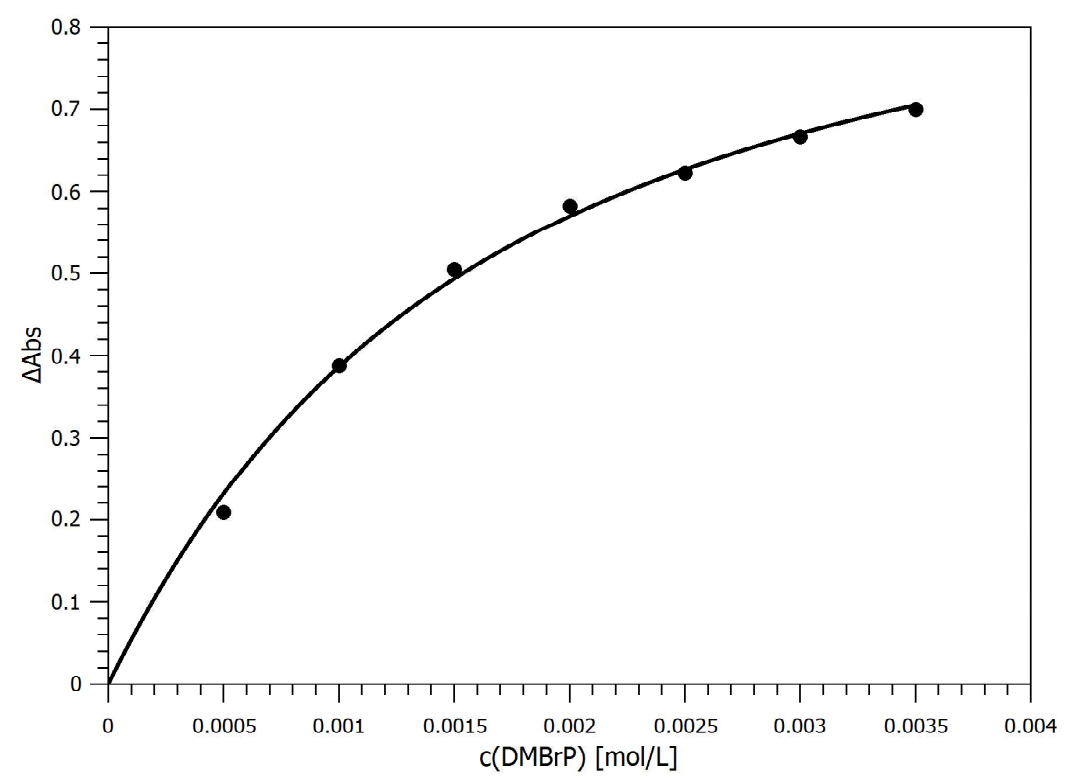

Figure 4.39 $\Delta \mathrm{Abs}$ vs DMBrP concentration and high affinity binding fit. MeCN, $c(\mathbf{1})=$ $5 \cdot 10^{-4} \mathrm{M}$ at $0{ }^{\circ} \mathrm{C}$. 
increasing concentration in DMBrP revealed a binding constant of $805 \mathrm{M}^{-1}$. This lies in the same regimen as the other investigated phenols such as TMP $\left(688 \mathrm{M}^{-1}\right)$.

\subsubsection{Analysis of the obtained polymer and mechanistic consid- erations}

To test the viability of the base assisted polymerization, different catalyst concentrations were tried. The influence of atmsopheric and inert conditions was tested, as well as control experiments with simple copper(II) salts were carried out. For this purpose, the resulting polymer was isolated and analyzed by NMR, GPC, MALDI-MS and TG-DSC measurements.

The reaction was carried out with 20, 50, 100 and 200 equivalents of monomer in MeCN solution with an excess of $\mathrm{Na}_{2} \mathrm{CO}_{3}$. Isolation of the polymer was done by evaporation to dryness under reduced pressure and subsequent extraction with THF to remove all phenolic material from the residue. The solubility of the polymer in THF was confirmed beforehand. The solvent was removed again and the residue washed with MeCN to remove remaining monomer and trace amounts of complex that may have remained in the THF solution.

An NMR experiment was performed to determine the nature of the polymeric strucure. The main peaks at $2.09 \mathrm{ppm}$ and $6.47 \mathrm{ppm}$ represent the $\mathrm{CH}_{3}$ group and the aromatic protons of the polymer. When zooming in on the peak at $2.09 \mathrm{ppm}$, more signals with lower intensity are found at 2.17, 2.14 and $2.04 \mathrm{ppm}$, which stem from the head and the tail of the polymer. Around the aromatic $\mathrm{C}-\mathrm{H}$ signal at $6.47 \mathrm{ppm}$ are two more signals at 6.45 and 6.36 that originate from the head and the tail of the polymer.

In conclusion, the polymer is obtained in good purity and most likely linear formed, as there are only two ${ }^{1} \mathrm{H}-\mathrm{NMR}$ signals for the chain from two independent positions that are also present in the monomer.

\section{GPC measurement}

After a first insight into the structure of the polymer by NMR spectroscopy, further investigations by means of GPC analysis were undertaken to determine the molecular mass of the polymer and its distribution. GPC experiments were run in THF as the eluent at room temperature with IR detection at $254 \mathrm{~nm}$. Calibration of the GPC was performed with polystyrene. Toluene was added to each sample as an internal reference. Each experiment was run twice. Mark-Houwink-parameter for the investigated polymer did not exist for THF in the literature, therefore no further adjustments were made to the obtained molecular mass distributions. The results are listed in Table 4.3. The first band to appear in the spectrum originates from added toluene that serves as an internal 

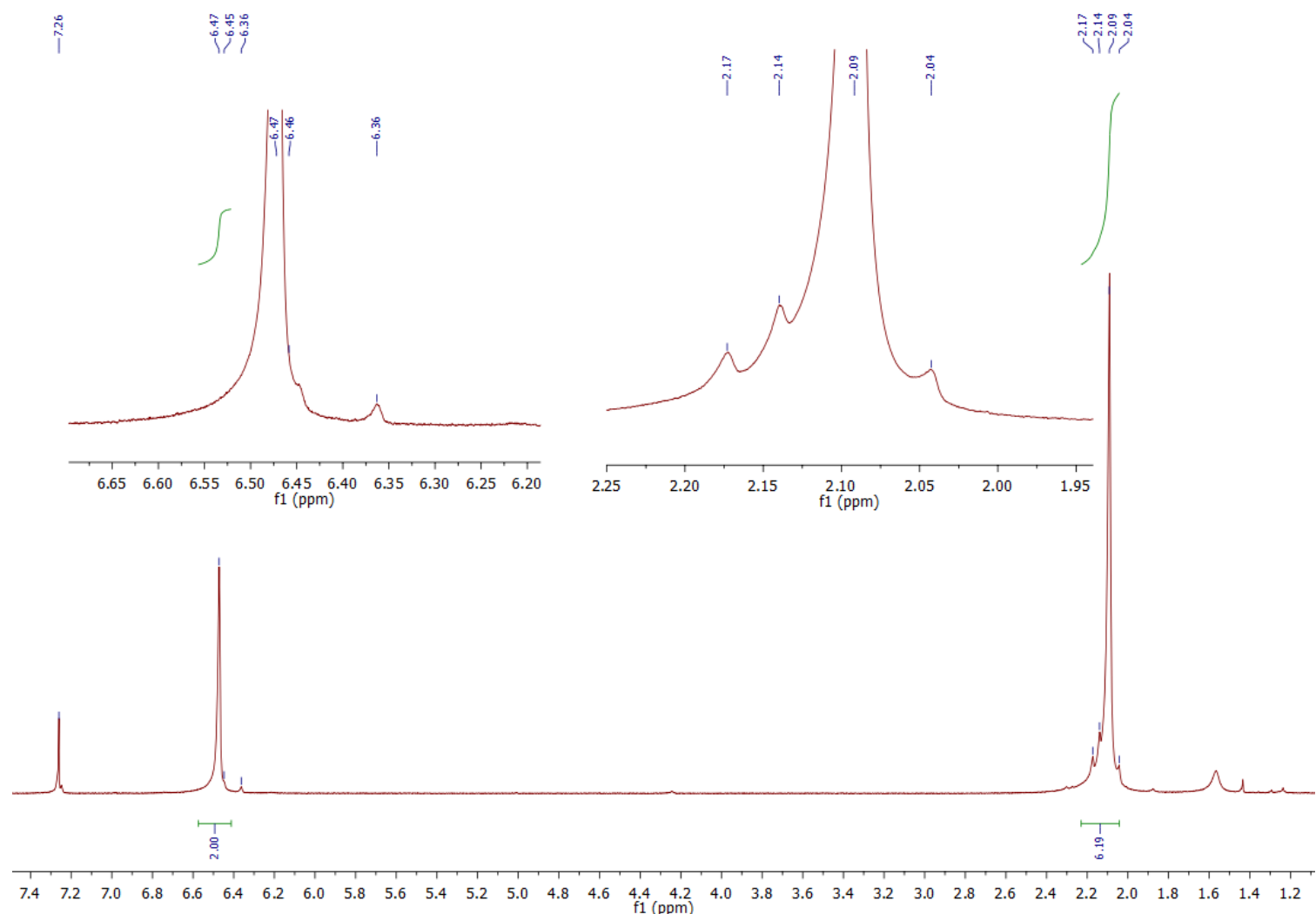

$\begin{array}{llllllllllllllllllllllllllllllllll}7.4 & 7.2 & 7.0 & 6.8 & 6.6 & 6.4 & 6.2 & 6.0 & 5.8 & 5.6 & 5.4 & 5.2 & 5.0 & 4.8 & 4.6 & 4.4 & 4.2 & 4.0 & 3.8 & 3.6 & 3.4 & 3.2 & 3.0 & 2.8 & 2.6 & 2.4 & 2.2 & 2.0 & 1.8 & 1.6 & 1.4 & 1.2\end{array}$

Figure 4.40 ${ }^{1} \mathrm{H}-\mathrm{NMR}$ spectrum in $\mathrm{CDCl}_{3}$ of the obtained polymer from a reaction of 50 eq. DMBrP and an excess of $\mathrm{Na}_{2} \mathrm{CO}_{3}$ with $\mathbf{1}$ in $\mathrm{MeCN}$ at roomtemperature. Insets show enlargemant of the areas aorund 2.10 and $6.46 \mathrm{ppm}$.

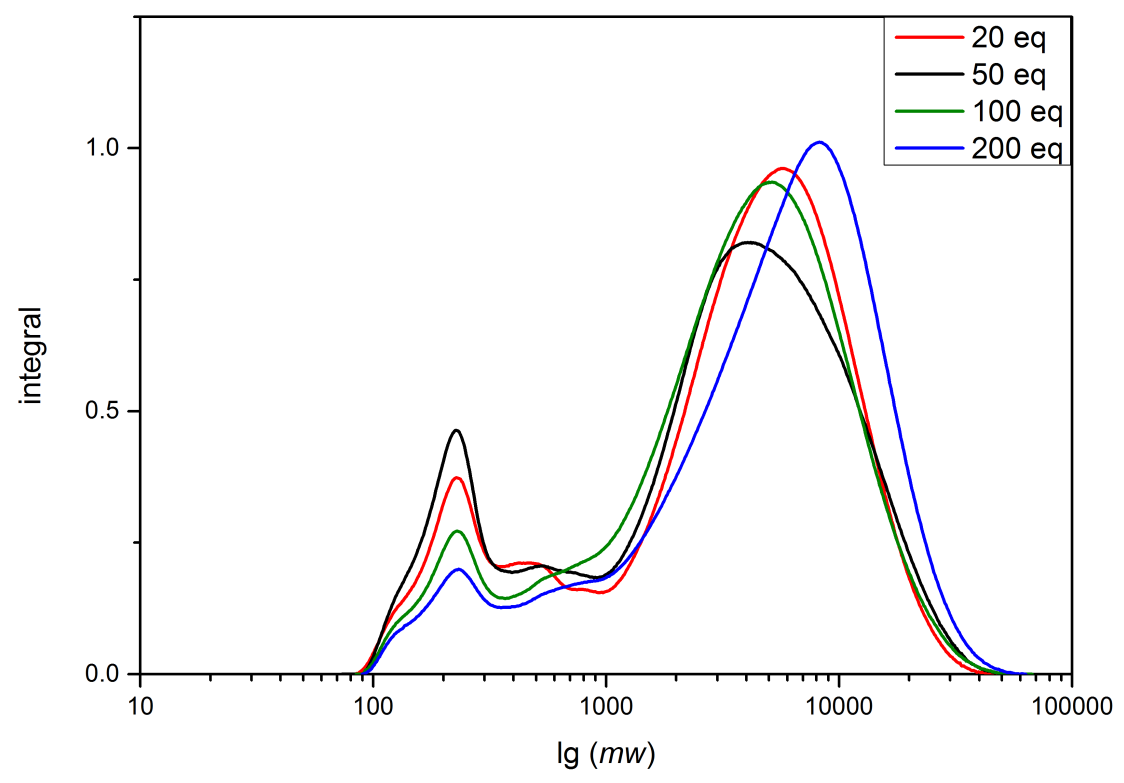

Figure 4.41 Molecular mass distribution of different polymerization attempts with different equivalents of substrate in $\mathrm{MeCN}$ at roomtemperature with an excess of $\mathrm{Na}_{2} \mathrm{CO}_{3}$, obtained from GPC measurement. 
Table 4.3 Obtained values by GPC analysis for different equivalents of substrate.

\begin{tabular}{ccccc}
$\begin{array}{c}\text { equivalents } \\
\text { DMBrP }\end{array}$ & $\begin{array}{c}M_{\mathrm{n}} \\
{[\mathrm{kg} / \mathrm{mol}]}\end{array}$ & $\begin{array}{c}M_{\mathrm{w}} \\
{[\mathrm{kg} / \mathrm{mol}]}\end{array}$ & $\begin{array}{c}M_{\mathrm{z}} \\
{[\mathrm{kg} / \mathrm{mol}]}\end{array}$ & $\mathrm{PDI}$ \\
\hline \hline \multirow{2}{*}{20} & 1.65 & 6.08 & 10.4 & 3.68 \\
\cline { 2 - 5 } & 2.92 & 9.53 & 16.7 & 3.26 \\
\hline \multirow{2}{*}{50} & 1.36 & 6.06 & 12.1 & 4.46 \\
\hline \multirow{2}{*}{100} & 1.86 & 8.18 & 16.3 & 4.40 \\
\hline \multirow{2}{*}{200} & 1.86 & 6.02 & 11.2 & 3.24 \\
\cline { 2 - 5 } & 1.36 & 7.50 & 14.8 & 5.51 \\
\hline & 2.25 & 8.03 & 13.4 & 3.19 \\
\hline
\end{tabular}

references for the spectrometer. Peaks at around 220 and $480 \mathrm{~g} \cdot \mathrm{mol}^{-1}$ most likely stem from di- and tetramers that have not been removed by the washing process. The number

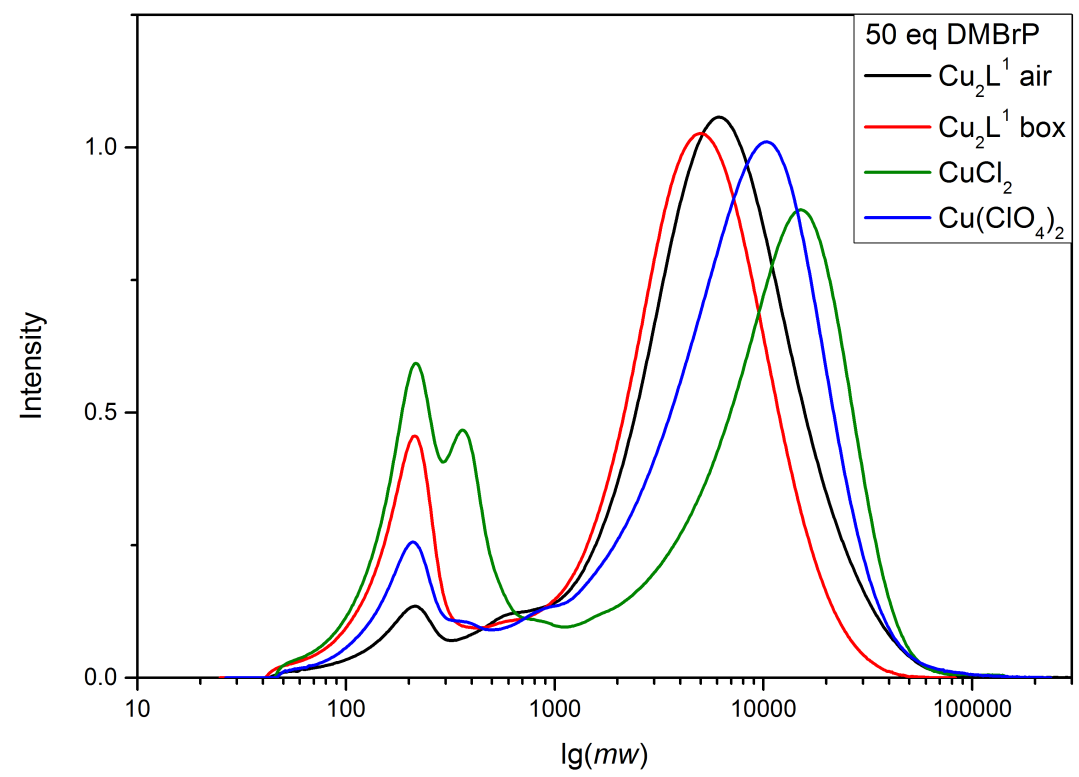

Figure 4.42 Molecular mass distribution for the reaction of 50 equivalents of DMBrP and an excess of $\mathrm{Na}_{2} \mathrm{CO}_{3}$ with 1 under ambient and anaerobic conditions and different metal salts under ambient conditions, determined by GPC analysis.

average molar mass $\left(M_{\mathrm{n}}\right)$ varies little in correlation to the equivalents used, while the two runs with the same amount of substrate differ more from one run to the other. The mass average molar mass $\left(M_{\mathrm{w}}\right)$ varies little for different equivalents of monomer. The molar mass average $\left(M_{\mathrm{z}}\right)$ also varies very little all in all. Therefore, an influence of the equivalents on the reaction is negligible within the investigated range. Since different equivalents do not influence the reaction, different conditions have been tested and compared to simple metal salts to verify the viability of use of $\mathbf{1}$ as the polymerization catalyst for DMBrP.

The reaction was carried out in a glovebox to investigate the influence of dioxygen and 
Table 4.4 Results of GPC analysis of the polymerization reaction with 50 equivalents of $\mathrm{DMBrP}$ under different conditions.

\begin{tabular}{lllll} 
conditions & $M_{\mathrm{n}}[\mathrm{kg} / \mathrm{mol}]$ & $M_{\mathrm{w}}[\mathrm{kg} / \mathrm{mol}]$ & $M_{\mathrm{z}}[\mathrm{kg} / \mathrm{mol}]$ & PDI \\
\hline \hline atmosphere & 1.86 & 8.18 & 16.3 & 4.40 \\
\hline inert & 1.10 & 6.53 & 10.8 & 3.51 \\
\hline $\mathrm{CuCl}_{2}$ & 1.15 & 11.8 & 20.6 & 10.3 \\
\hline $\mathrm{Cu}\left(\mathrm{ClO}_{4}\right)_{2}$ & 1.46 & 10.2 & 21.4 & 6.99
\end{tabular}

water on the reaction. The polymer obtained under inert conditions has a smaller molar mass, therefore a shorter chain length. The PDI of 3.51 is significantly smaller than of the reaction under atmospheric conditions. Although the catalyst cannot be reoxidized in the absence of dioxygen, if it was reduced during the reaction, the polymerization reaction takes place. Therefore, it is likely that the catalyst does not change its oxidation state during the reaction but rather acts as an initiator for the reaction, that can be of ionic or radical nature. The influence of water was considered responsible for the lower molecular weight and PDI.

Comparing the polymer obtained by using $\mathbf{1}$ as the catalyst and the polymer obtained with simple copper(II) salts as the catalst shows a decrease in $M_{\mathrm{n}}$ values while the PDI value goes up.

A control experiment with only $\mathrm{DMBrP}$ and $\mathrm{Na}_{2} \mathrm{CO}_{3}$ present did not undergo any reaction.

\section{MALDI-MS measurement}

The polymer obtained after the reaction of $\mathbf{1}$ and 200 equivalents of monomer was subjected to MALDI-MS analysis (Figure 4.43).

The spectrum shows equidistant peaks with decreasing intensity going to higher $\mathrm{m} / z$ values. The peaks are $120 \mathrm{~m} / z$ units apart which corresponds to a 2,6-dimethyl phenol unit of the polymer. The peak at $m / z=1402.6$ was chosen for simulation. The simulated isotopic pattern corresponds to a chain of $11 \mathrm{C}-\mathrm{O}$ connected monomer units with a bromo substituent on one end and a hydoxy group on the other end of the chain $\left(\mathrm{C}_{88} \mathrm{H}_{89} \mathrm{O}_{11} \mathrm{Br}\right)$.

\section{Resonance Raman}

A recorded resonance Raman spectrum $\left(\lambda_{\mathrm{ex}}=488.7 \mathrm{~nm}\right)$ of the adduct of DMBrP and $\mathbf{1}$ in frozen MeCN shows two strongly resonance enhanced peaks and a few weaker signals. The resonance enhanced transition is that of the LMCT band, which means the $\mathrm{C}-\mathrm{O}$ stretch has to be involved and was assigned to the band at $1278 \mathrm{~cm}^{-1}$. The other strong band at $1577 \mathrm{~cm}^{-1}$ is too high in energy for a $\mathrm{C}-\mathrm{O}$ stretch and might correspond to the $\mathrm{C}_{\text {ortho }}-\mathrm{C}_{\text {meta }}$ stretching frequency that is directly involved, although $1623 \mathrm{~cm}^{-1}$ signal 


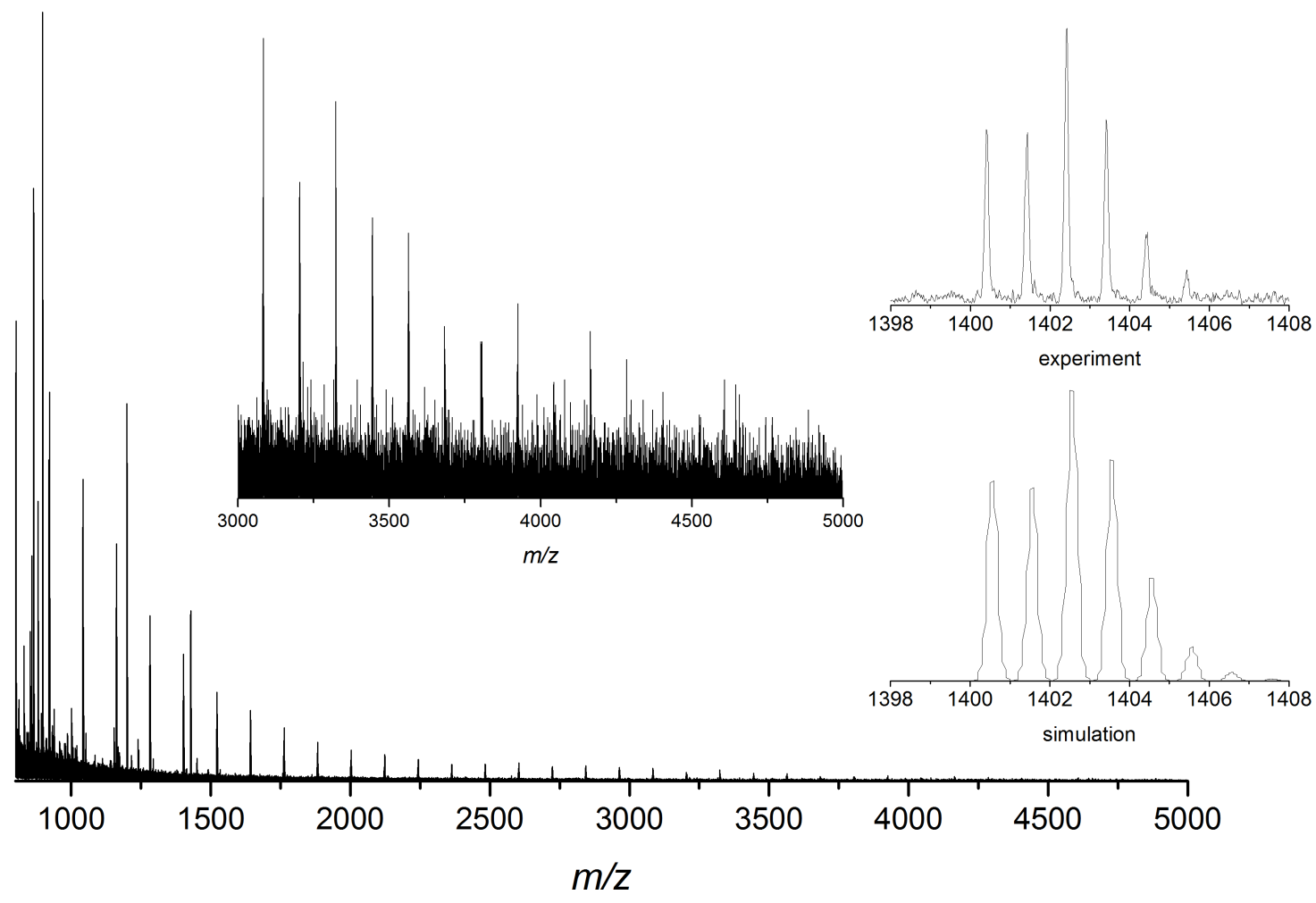

Figure 4.43 MALDI mass spectrum of the polymer obtained after reaction with 200 equivalents of DMBrP with 1 and an excess $\mathrm{Na}_{2} \mathrm{CO}_{3}$ in MeCN. Left inset shows enlargement of the region between $m / z=3000$ and 5000. Right inset shows enlargemant and simulation of a chain of $11 \mathrm{C}-\mathrm{O}$ connected units $\left(\mathrm{C}_{88} \mathrm{H}_{89} \mathrm{O}_{11} \mathrm{Br}\right)$.

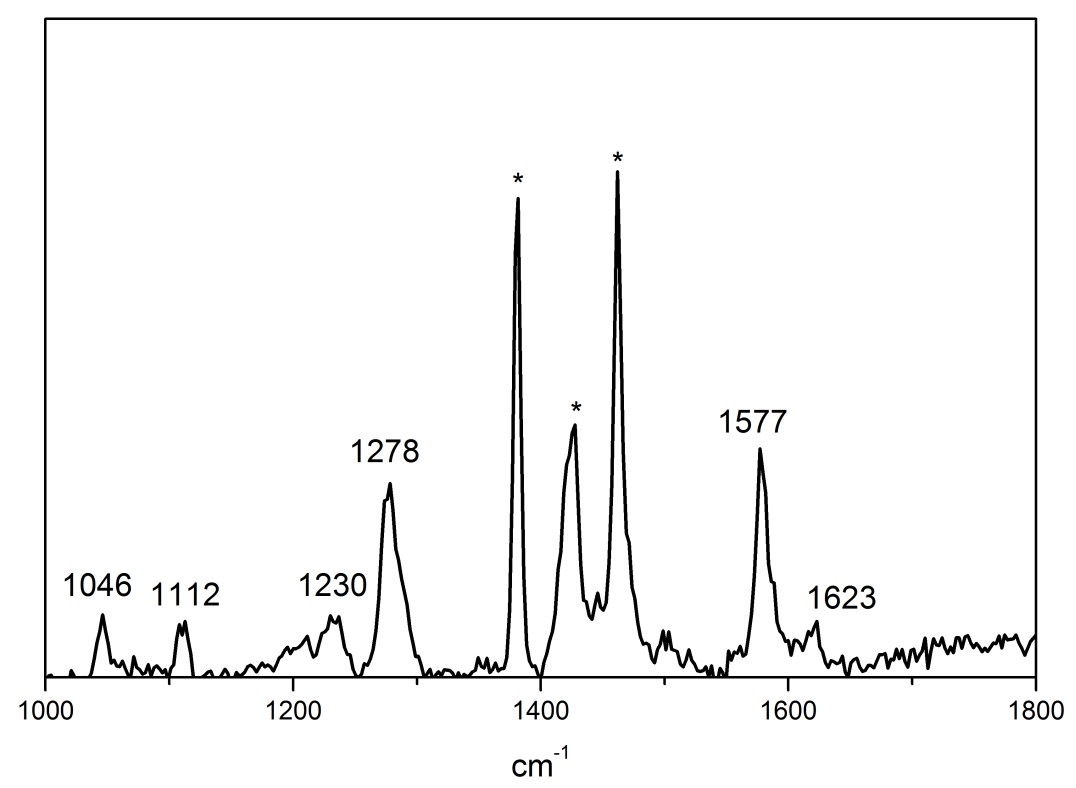

Figure 4.44 Resonance Raman spectrum of the adduct of DMBrP and 1 in frozen MeCN solution. Solvent signals are marked with an asterisk. 
would also fit, as it closer to the one observed for TMP $\left(1615 \mathrm{~cm}^{-1}\right)$.

With the $\mathrm{C}-\mathrm{O}$ stretch at $1278 \mathrm{~cm}^{-1}$ the nature of the transition seems to be a copper $(\mathrm{II}) \leftarrow$ phenolate transition as observed in all previous cases.

\section{EPR measurement}

The polymerization reaction of DMBrP catalyzed by $\mathbf{1}$ was run in a glovebox with and without additional base. After the reaction was completed, the solutions were transferred into EPR Young-tubes and frozen in liquid nitrogen until they were measured. The two
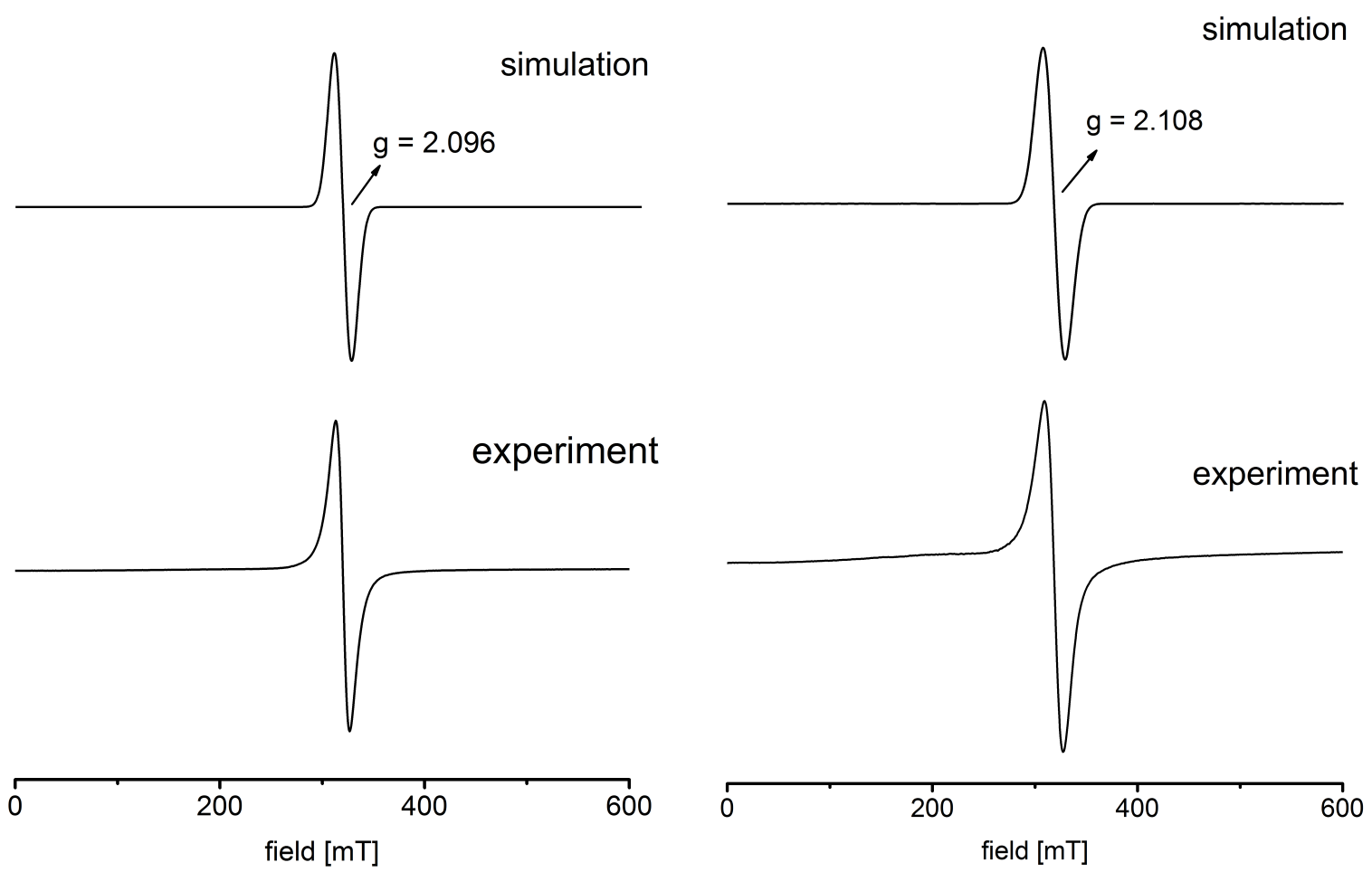

(a) EPR spectrum after the reacion of $\mathrm{DMBrP}$ and $\mathbf{1}$ is completed (simulation parameters: Nucleus $=\mathrm{Cu}$, $\mathrm{Cu}$, total spin $=1$, frequency $=9.430 \mathrm{GHz}$, linewidth $=8 \mathrm{mT}, \mathrm{g}_{\|}=\mathrm{g}_{\perp}=2.1, \mathrm{~A}=0.0136 \mathrm{~cm}^{-1}$, gStrain $=[0.1,0.1,0.05])$.

(b) EPR spectrum after the reaction with additional $\mathrm{Na}_{2} \mathrm{CO}_{3}$ is completed. (simulation parameters: Nucleus $=\mathrm{Cu}, \mathrm{Cu}$, total $\operatorname{spin}=1$, frequency $=9.432 \mathrm{GHz}$, linewidth $=8 \mathrm{mT}, \mathrm{g}_{\|}$ $=\mathrm{g}_{\perp}=2.108, \mathrm{~A}=0.0123 \mathrm{~cm}^{-1}, \mathrm{gStrain}=[0.1$, $0.1,0.05])$.

Figure 4.45 EPR spectra after the reaction of DMBRP and 1 in MeCN at $160 \mathrm{~K}$ under different conditions.

spectra recorded at $160 \mathrm{~K}$ do not exhibit any significant difference. They both show a broad signal at $320 \mathrm{mT}$ with a g-value of around 2.1. A similar g-value was observed for other dicopper complexes of $\mathbf{H L}^{1}$. Due to its broadness and a g-value deviating from 2.0 this signal does not seem to be of organic nature, but rather the unoxidized dicopper(II) species, although the half field signal typical for the complex is not seen in the spectrum indicating a $S=1 / 2$ system due to the loss of antiferromagnetic coupling between the copper(II) ions. A mixed valent copper(I)/copper(II) species, localized on the EPR time 
scale, showed a four-line pattern typical for a mononuclear copper(II) complex. The A values obtained by simulating the spectra are $0.0004 \mathrm{~cm} 1-1$ for the sample containing $\mathrm{Na}_{2} \mathrm{CO}_{3}$ and $0.0020 \mathrm{~cm} 1-1$ for the sample without additional base.

An EPR spectrum (not shown) measured during the reaction looks exactly like the ones measured after the reaction. So, there does not seem to be any involvement of a radical species or reduction of the complex. So it is likely that the reaction proceeds via an ionic pathway with the complex simply enabling nucleophilic substitution of a bound bromo phenol, as hinted at by the liberation of $\mathrm{HBr}$ during the reaction.

\section{$T G$-DSC measurement}

The TG-DSC measurement of the polymer obtained after the reaction of DMBrP with 1 and an excess of $\mathrm{Na}_{2} \mathrm{CO}_{3}$ in $\mathrm{MeCN}$, was carried out under nitrogen atmosphere in an open $\mathrm{Al}_{2} \mathrm{O}_{3}$ vessel. The TG measurement reveals a thermal stability up to $450{ }^{\circ} \mathrm{C}$ before

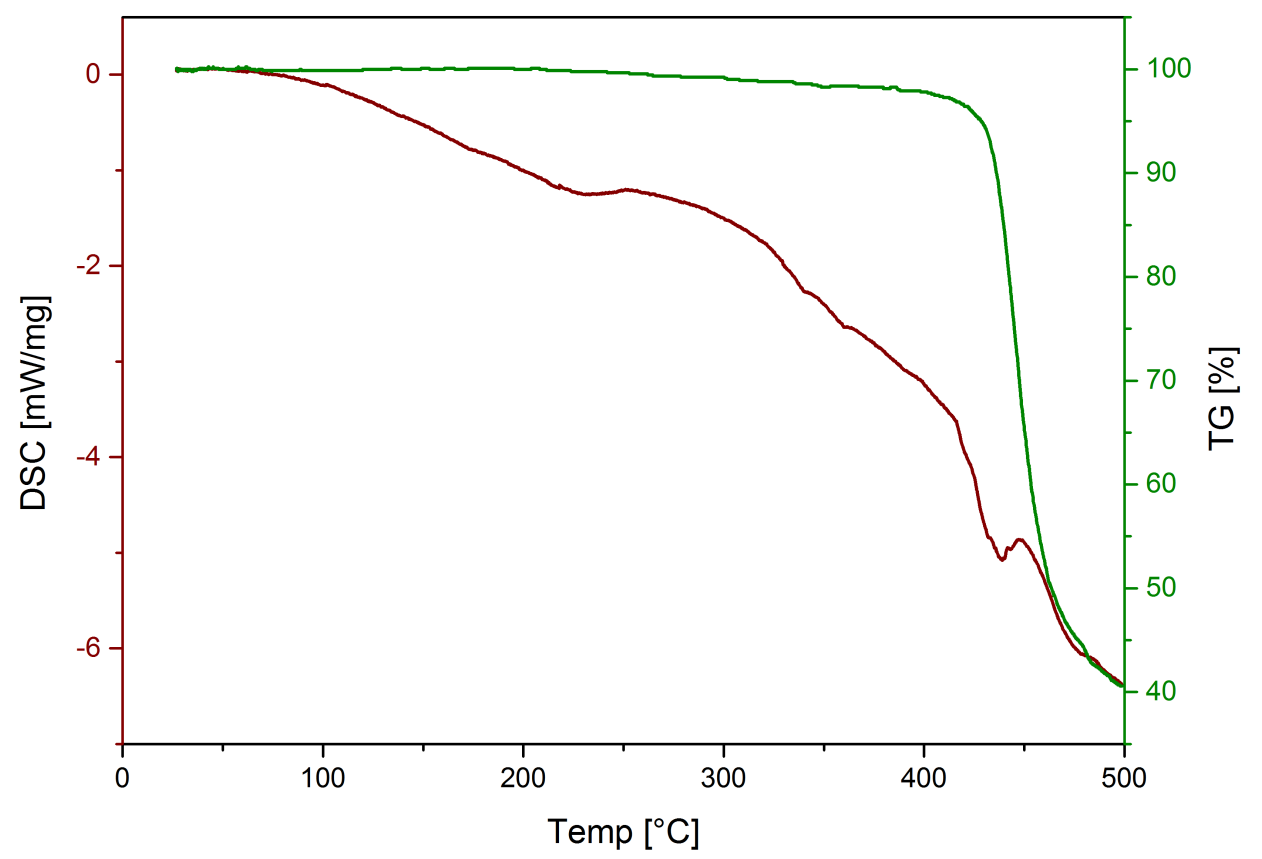

Figure 4.46 Combined TG and DSC spectra of the obtained 2,6-dimethyl polyphenylene oxide.

thermal decomposition.

From 50 to $220{ }^{\circ} \mathrm{C}$ a constant decrease in heatflow is observed until a local minimum is reached at $220^{\circ} \mathrm{C}$ that is attributed to the melting of the polymer. The literature reported melting point is found at a slightly higher value of $265{ }^{\circ} \mathrm{C}$. Until $410{ }^{\circ} \mathrm{C}$ a stronger decrease and a local maximum at $450{ }^{\circ} \mathrm{C}$ is observed when the sample starts to decompose.

Literature values for the glass transition temperature are around $210{ }^{\circ} \mathrm{C}$. 193 A glass transition is not observed in the spectrum at hand. 
In conclusion, it was possible to run the polymerization of 4-bromo-2,6-dimethylphenol catalytically with $\mathbf{1}$ as the catalyst and to gain insight into the polymerization mechanism. The obtained polymer shows a molecular weight that lies one order of magnitude below industrial values. The PDI of around 4 is higher than for industrial processes that reach a value of 2 . Nevertheless the polymer can still be useful for purposes where a higher $\mathrm{PDI}$ is required.

A control experiment with simple metal salts as the catalyst revealed that they are also able to catalayze the reaction but yield an even broader molecular mass distribution.

\subsubsection{Conclusion}

The mechanism behind the catalytic oxidative para-C-C coupling of TMP was further elucidated. It was possible to determine the oxidation states of all species involved in the process via EPR and UV/Vis spectroscopy and the fate of the $\mathrm{O}_{2}$ mlecule involved in the reoxidation of the mixed valent complex species was determined by oxygen uptake experiments. Furthermore, it was possible to expand the reactivity to catalytic functionalization of the para- $\mathrm{CH}_{3}$ group of TMP mediated by $\mathbf{1}$ to give HMP, MDP or HDB depending on the solvent an the reaction condictions. Binding stoichiometries, binding modes and binding constants were determined by means of UV/VIs and resonance Raman spectroscopy. Addtionally the reaction conditions for the polymerization of $\mathrm{DMBrP}$ through $\mathrm{C}-\mathrm{O}$ coupling catalyzed by $\mathbf{1}$ were optimized and the obtained polymer was characterized by means of ${ }^{1} \mathrm{H}-\mathrm{NMR}$ spectroscopy, Gel permeation chromatography as well as thermogravimetric analysis. 



\section{Structure and Reactivity of $\mathrm{L}^{2} \mathrm{Cu}_{2}^{\mathrm{II}}\left(\mathrm{NO}_{3}\right)_{3}$ and $\mathrm{L}^{2} \mathrm{Cu}_{2}^{\mathrm{I}} \mathrm{OTf}$}

The compartmental binucleating ligand $\mathbf{H L}^{2}$ is based on the $\mathbf{L 6 6}$ ligand synthesized by CASELla and was expected to form complexes of similar structure and reactivity. 176 . S.GHosh synthesized the corresponding dicopper(II) and dicopper(I) complexes and started preliminary spectroscopic studies, as well as their reactivity with sodium 2,4,6trimethylphenolate.

\subsection{Spectroscopic properties of dicopper complexes of $\mathrm{HL}^{2}$}

GHOSH carried out first attemps to synthesize a dicopper(II) complex of $\mathbf{H L}^{2}$ in $\mathrm{MeCN}$ with one equivalent of $\mathrm{KO}^{t} \mathrm{Bu}$ and two equivalents $\mathrm{Cu}^{\mathrm{II}}(\mathrm{OTf})_{2}$.

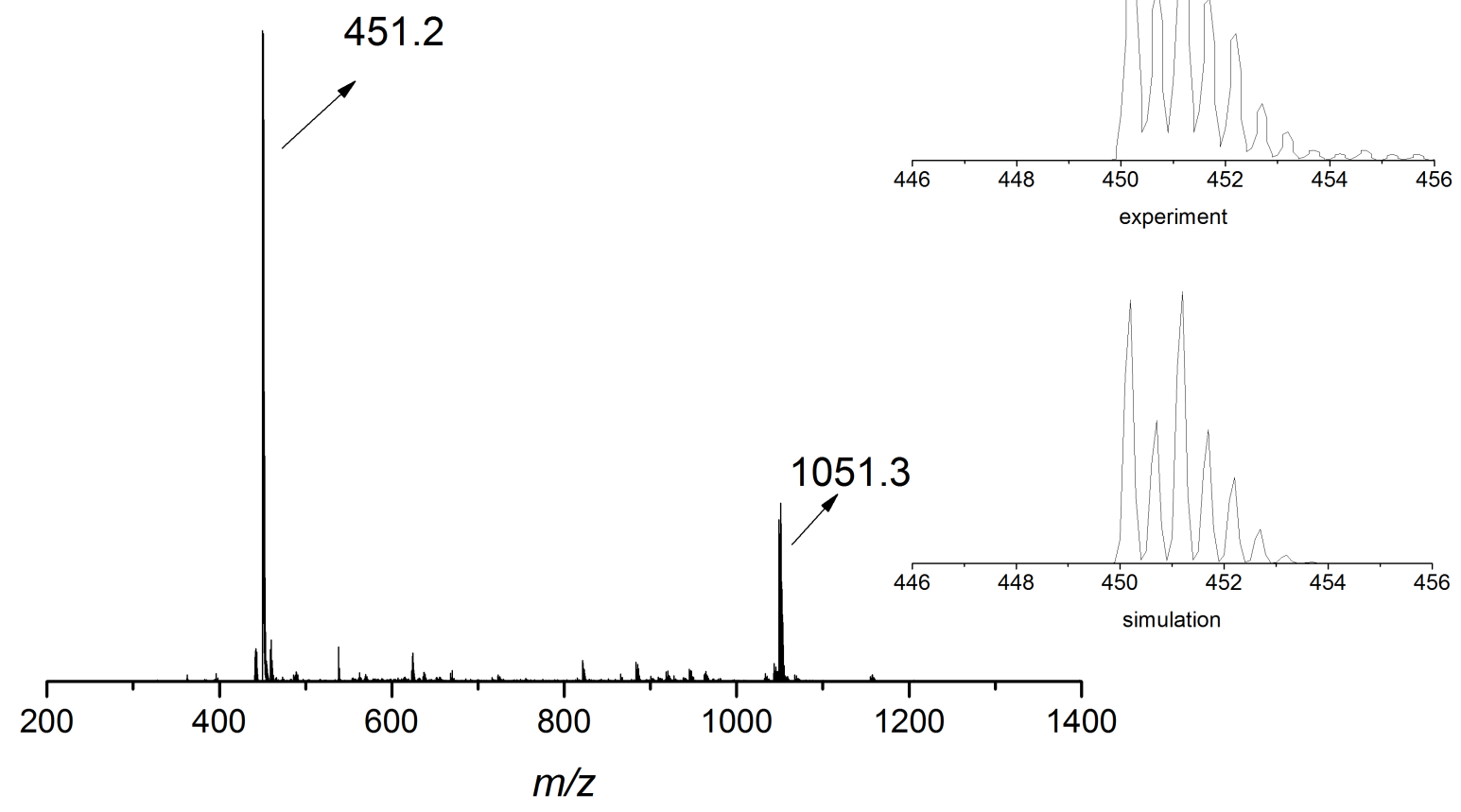

Figure 5.1 Positive ESI-MS spectrum of $\left[\mathbf{L}^{2} \mathrm{Cu}_{2}^{\mathrm{II}} \mathrm{OH}\right](\mathrm{OTf})_{2}$ in $\mathrm{MeCN}$. The inset shows enlargement of the peak at $m / z=451.2$ and the simulated pattern of $\left[\mathbf{L}^{2} \mathrm{Cu}_{2}^{\mathrm{II}} \mathrm{OH}\right]^{2+}$.

GHOSH first confirmed its formation formation by ESI mass spectrometry (Figure 5.1). The ESI mass spectrum shows two major peaks, which both belong to a dicopper species. 
The peak at $m / z=451.2$ corresponds to $\left[\mathrm{L}^{2} \mathrm{Cu}_{2}(\mathrm{OH})\right]^{2+}$ and the one at $m / z=1051$ is the singly charged species with an additional triflate ion, $\left[\mathbf{L}^{2} \mathrm{Cu}_{2}(\mathrm{OH})(\mathrm{OTf})\right]^{+}$.

For the purpose of purification and crystallization it was beneficial to change to $\mathrm{Cu}^{\mathrm{II}}\left(\mathrm{NO}_{3}\right)_{2} \cdot 3 \mathrm{H}_{2} \mathrm{O}$ as the source of copper. GHOSH achieved crystallization by slow diffusion of diethyl ether into a solution of $\left[\mathbf{L}^{2} \mathrm{Cu}_{2}^{\mathrm{II}}\left(\mathrm{NO}_{3}\right)_{2}\right]\left(\mathrm{NO}_{3}\right)$ in $\mathrm{MeCN}$ to give green, block shaped crystals. Two structures relevant to this work were obtained. The first one is depicted in Figure 5.2 with nitrate anions coordinated to the two copper ions and the second structure (Figure 5.3) with an internal water/hydroxide bridge.

In the molecular structure of $\mathbf{4}$, the two copper ions are each five-fold coordinated in a distorted square pyramidal fashion. Four nitrogen donors from the ligand framework and two oxygen donors from a coordinating nitrate make up the coordination environment. Since nitrates are weakly coordinating, they will likely be substituted by coordinating solvent molecules in solution, enabling the complex to bind a substrate.

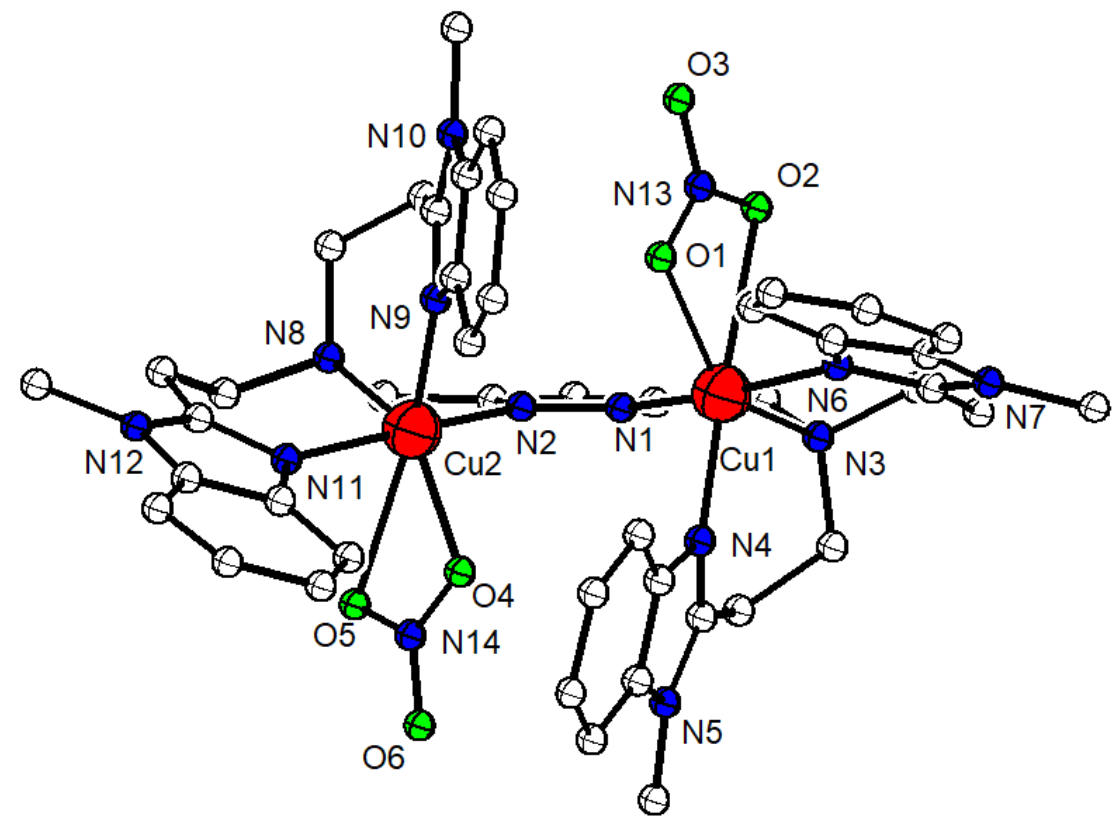

Figure 5.2 View along the z-axis of the molecular structure of $\left[\mathrm{L}^{2} \mathrm{Cu}_{2}^{\mathrm{II}}\left(\mathrm{NO}_{3}\right)_{2}\right]\left(\mathrm{NO}_{3}\right)$ (4). Thermal displacement ellipsoids are drawn at 50\%. Anions and hydrogen atoms are omitted for clarity

The copper-copper distance measures $4.19 \AA$ with the two atoms diverging each by around $11^{\circ}$ from the plane of the pyrazole ring. The copper-copper distance in structure $\mathbf{5}$ measures $4.36 \AA$ and is slightly longer compared to $\mathbf{4}$ but the same as in $\mathbf{1}$. The distance of the oxygen atoms measures $2.49 \AA$ with an $\mathrm{O}-\mathrm{H}-\mathrm{O}$ angle of $151.7^{\circ}$ compared to $2.41 \AA$ and $170.6^{\circ}$ in $\mathbf{1}$.

Since only the crystallization of $\left[\mathrm{L}^{2} \mathrm{Cu}_{2}^{\mathrm{II}}\left(\mathrm{NO}_{3}\right)_{2}\right]\left(\mathrm{NO}_{3}\right)$ was reproducible, further investi- 
gations were carried out with this complex.

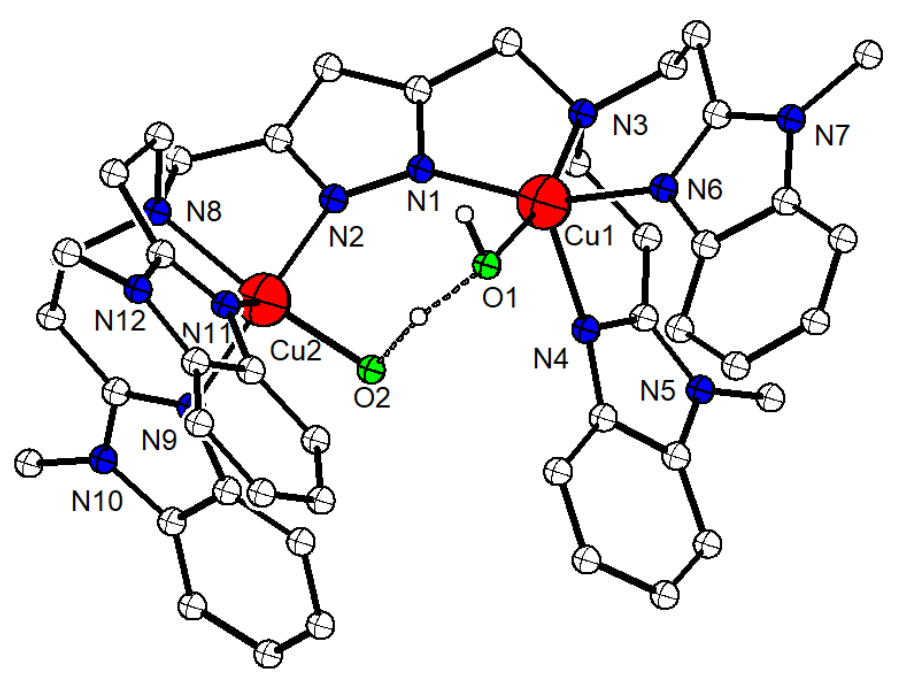

Figure 5.3 Molecular structure of $\left[\mathbf{L}^{2} \mathrm{Cu}_{2}^{\mathrm{II}}\left(\mathrm{H}_{2} \mathrm{O}\right)(\mathrm{OH})\right]\left(\mathrm{NO}_{3}\right)_{2}(\mathbf{5})$. Thermal displacement ellipsoids are drawn at 50\%. Anions and hydrogen atoms are omitted for clarity.

\section{$U V /$ Vis spectroscopy}

$\mathrm{UV} /$ Vis spectroscopic analysis of $\mathbf{4}$ showed a typical spectrum for a copper(II)-amine complex. There is a strong absorption below $400 \mathrm{~nm}$ without a clear maximum, caused by $\pi-\pi^{*}$ transistions of the ligand framework and LMCT transitions involving $\left[\mathbf{L}^{2}\right]^{-}$. Futhermore, a broad $d-d$ band at $670 \mathrm{~nm}$ is visible (Figure 5.4).

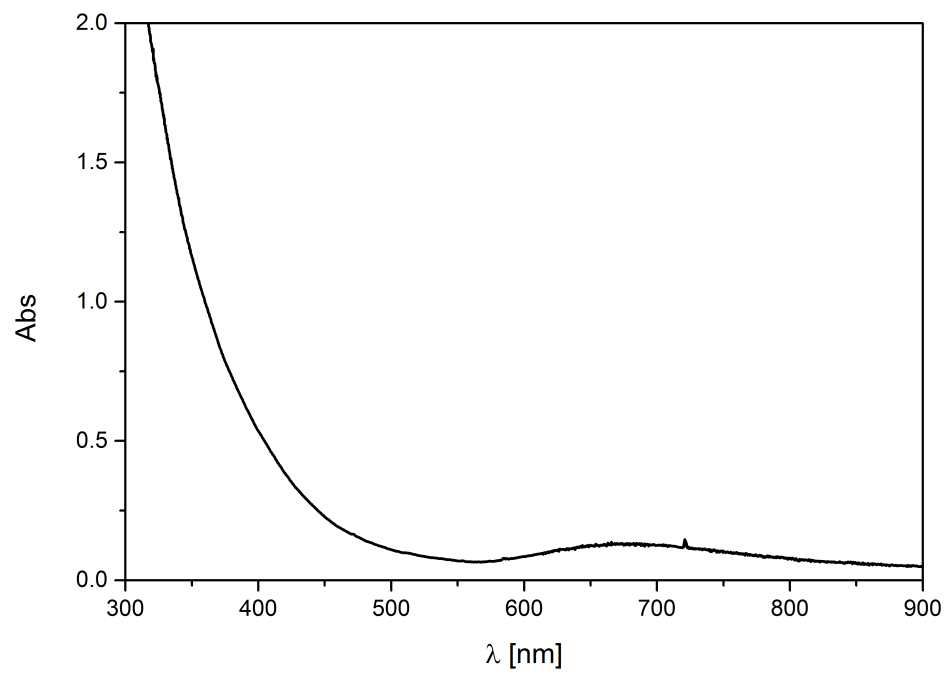

Figure 5.4 UV/Vis spectrum of $\left[\mathrm{L}^{2} \mathrm{Cu}_{2}^{\mathrm{II}}\left(\mathrm{NO}_{3}\right)_{2}\right]\left(\mathrm{NO}_{3}\right)\left(c=10^{-3} \mathrm{M}\right)$ in $\mathrm{MeCN}$ at room temperature.

Magnetic measurement

A variable temperature magnetic measurement from 2 to $300 \mathrm{~K}$ of $\mathbf{4}$ revealed a strong 
antiferromagnetic coupling between two $S=1 / 2$ systems, with a coupling constant of $J=-28 \mathrm{~cm}^{-1}$, which is lower than that of $\mathbf{1}$ with a coupling constant of $J=-35 \mathrm{~cm}^{-1}$ (Figure 5.5).

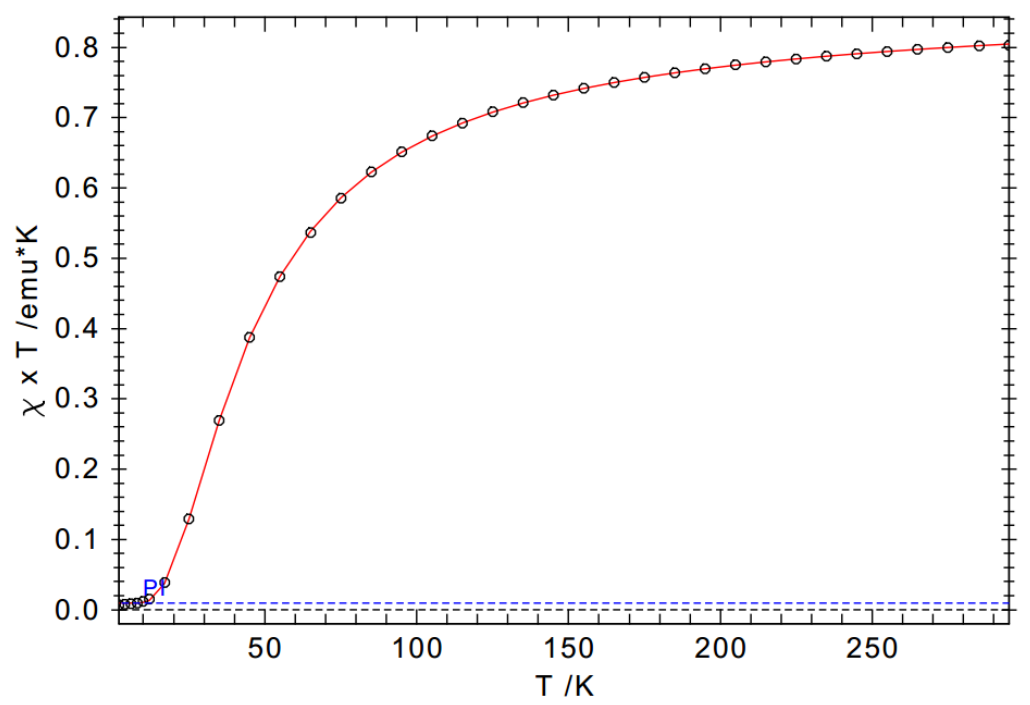

Figure 5.5 $\chi_{m} T$ vs $T$ measurement from $2 \mathrm{~K}$ to $300 \mathrm{~K}$ (circles) and fit (red line) of $\mathbf{4}$, suggestive of aniferromagnetic coupling between two $S=1 / 2$ systems.

\section{EPR Spectroscopy}

Furthermore, EPR spectroscopy of $\mathbf{4}$ performed in frozen MeCN glass at $150 \mathrm{~K}$ revealed a single broad signal at $320 \mathrm{mT}$ that corresponds to a g-value of 2.15 (Figure 5.6), that was also observed for $\mathbf{1}$.

\subsubsection{Synthesis of the dicopper(I)complex}

The dicopper(I) complex was synthesized in a glovebox by deprotonation of the precursor $\mathrm{HL}^{2}$ and subsequent addition of two equivalents of copper(I) triflate. $\left[\mathbf{L}^{2} \mathrm{Cu}_{2}^{\mathrm{I}}\right] \mathrm{OTf}(\mathbf{6})$ was investigated by NMR spectroscopy and ESI mass spectrometry.

ESI mass spectrometry revealed the formation of one major species (Figure 5.7), namely $\left[\mathrm{L}^{2} \mathrm{Cu}_{2}^{\mathrm{I}}\right]^{+}(m / z=883.3)$. The second peak at $m / z=443.2$ was assigned to the same species with an additional proton, $\left[\mathrm{L}^{2} \mathrm{Cu}_{2}^{\mathrm{I}} \mathrm{H}\right]^{2+}$. NMR signals at 8.44, 7.35, 7.16 and 6.95 ppm were assigned to the aromatic protons of the benzimidazole ring (Figure 5.8). All signals exhibit well resolved coupling patterns and show an equivalency in solution of all four benzimidazole sidearms. The single proton resonating at $5.83 \mathrm{ppm}$ corresponds to the hydrogen on the pyrazole. The backbone $\mathrm{CH}_{2}$ groups can be found at $3.68 \mathrm{ppm}$ and the $\mathrm{CH}_{2}$ groups of the side arms at 2.95 and $2.87 \mathrm{ppm}$. 


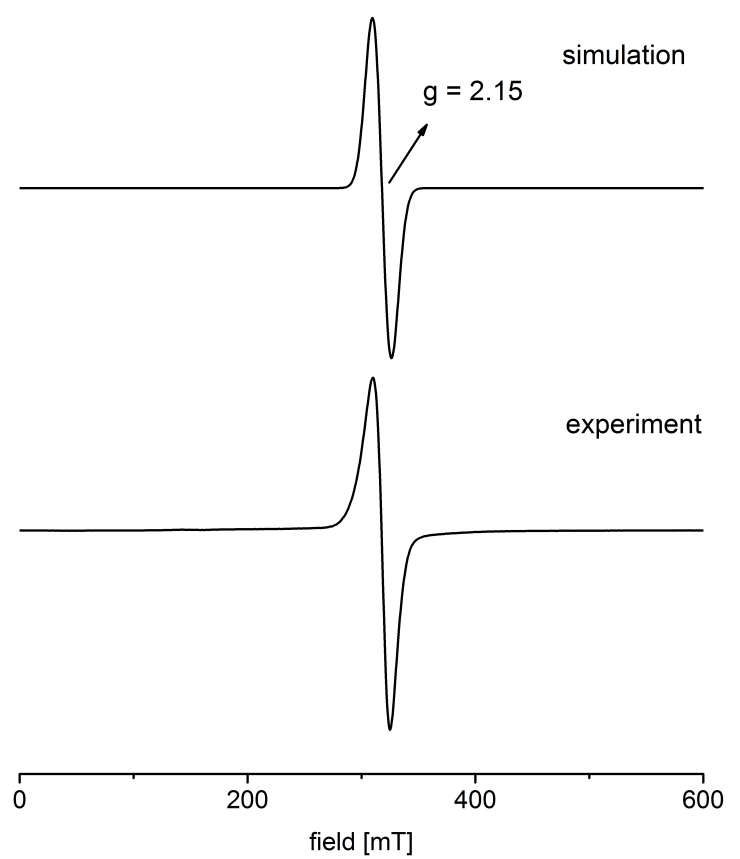

Figure 5.6 EPR spectrum of 4 in MeCN recorded at $150 \mathrm{~K}$ and $9.430 \mathrm{GHz}$. Simulation gives a g-value of 2.15 and an A-value of $0.0040 \mathrm{~cm}^{-1}$ (additional parameters: nucleus $=$ $\mathrm{Cu}, \mathrm{Cu}$, total $\mathrm{spin}=1, \mathrm{~T}=150 \mathrm{~K}$, linewidth $=8 \mathrm{mT}$, frequency $=9.430 \mathrm{GHz}$, gstrain $=$ $[0.2,0.1,0.05])$. 


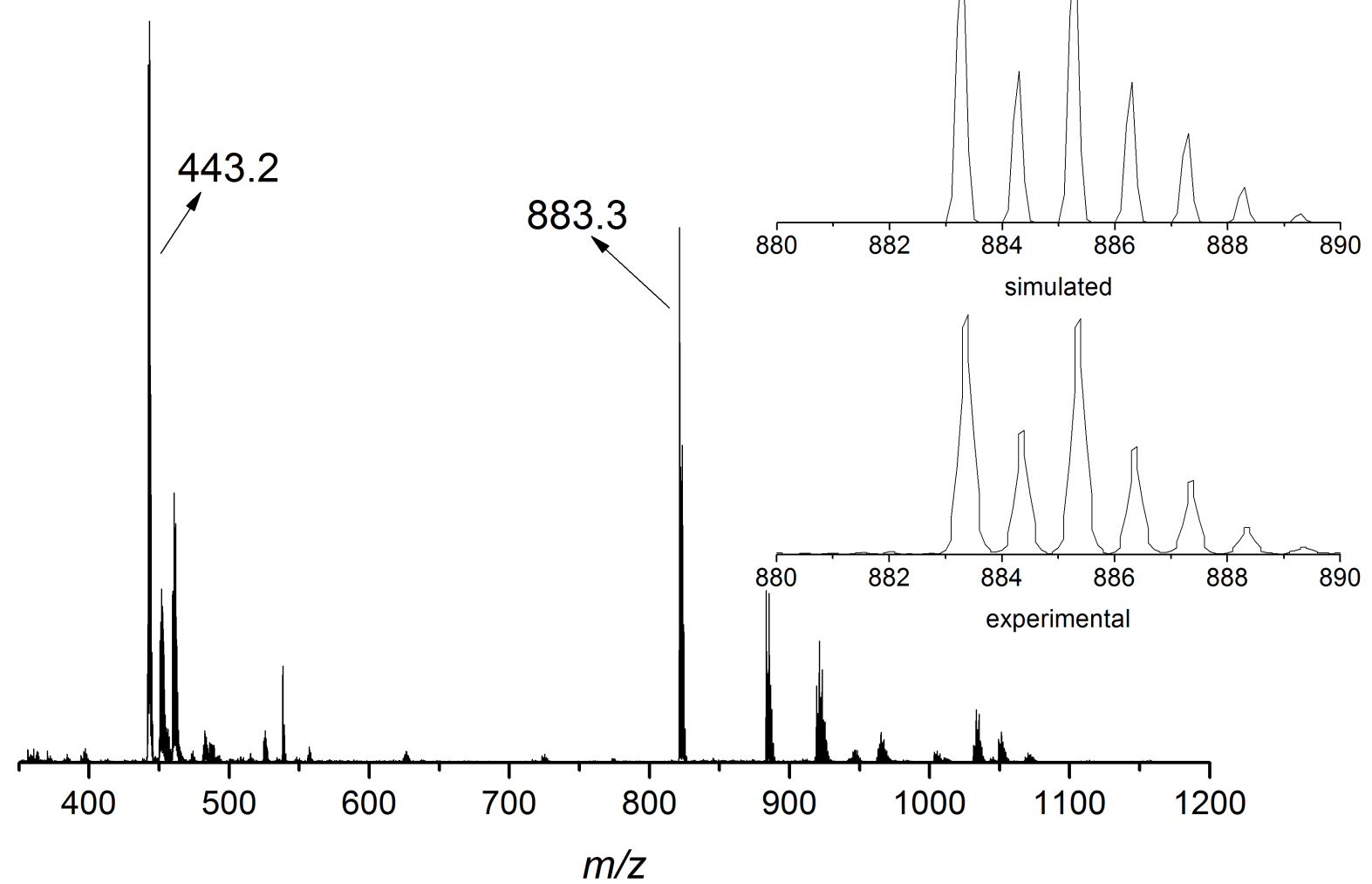

Figure 5.7 ESI-MS spectrum of $\left[\mathrm{L}^{2} \mathrm{Cu}_{2}^{\mathrm{I}}\right] \mathrm{OTf}$ in MeCN solution. Major peaks were assigned to the main compound. Inset shows enlargement of the peak at $\mathrm{m} / z=883.3$ and coresponding simulation of $\left[\mathbf{L}^{2} \mathrm{Cu}_{2}^{\mathrm{I}}\right]^{+}$.

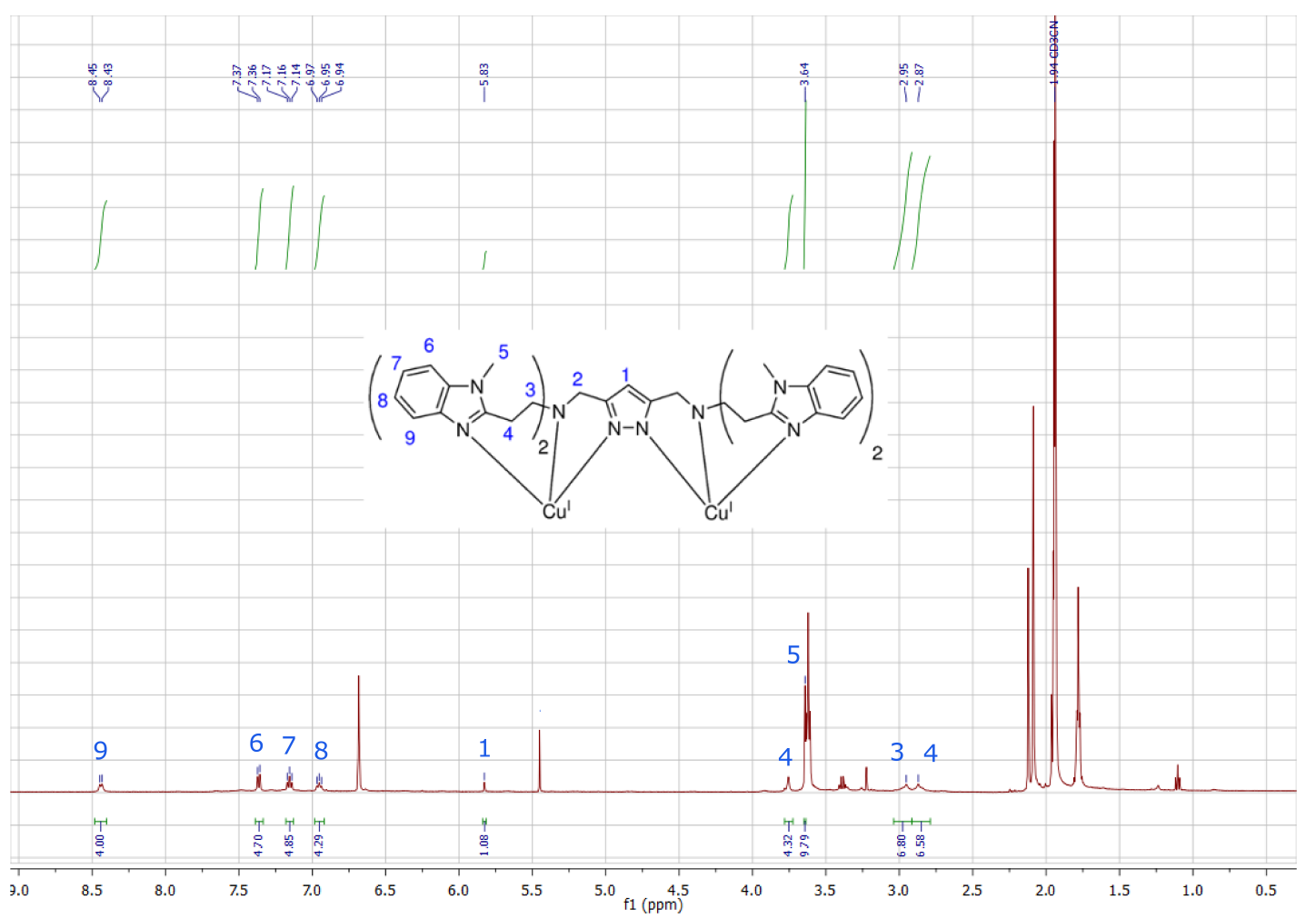

Figure $5.8{ }^{1} \mathrm{H}$ NMR spectrum of $\left[\mathbf{L}^{2} \mathrm{Cu}_{2}^{\mathrm{I}}\right] \mathrm{OTf}$ in $\mathrm{CD}_{3} \mathrm{CN}$ at $-30{ }^{\circ} \mathrm{C}$. 


\subsection{Reactivity of the dicopper complexes of $\mathrm{HL}^{2}$}

\subsubsection{Reactivity of $\left[\mathrm{L}^{2} \mathrm{Cu}_{2}^{\mathrm{I}}\right] \mathrm{OTf}$ with dioxygen}

When 6 was dissolved in dry, degassed EtCN at $-80{ }^{\circ} \mathrm{C}$ and exposed to dry dioxygen a transient signal at $560 \mathrm{~nm}$, along with a small shoulder at $650 \mathrm{~nm}$ characteristic for a trans-peroxo intermediate and a new band at $800 \mathrm{~nm}$ assigned to a $d$ - $d$ transition were observed (Figure 5.9). After 30 seconds, the band disappeard and a new band at $645 \mathrm{~nm}$ appeared along with a colour change from light purple to green, indicating an oxidation of the copper(I) ions to copper(II). Due to its short lifespan of only a few seconds at -80 ${ }^{\circ} \mathrm{C}$, the reactive intermediate was not studied further.

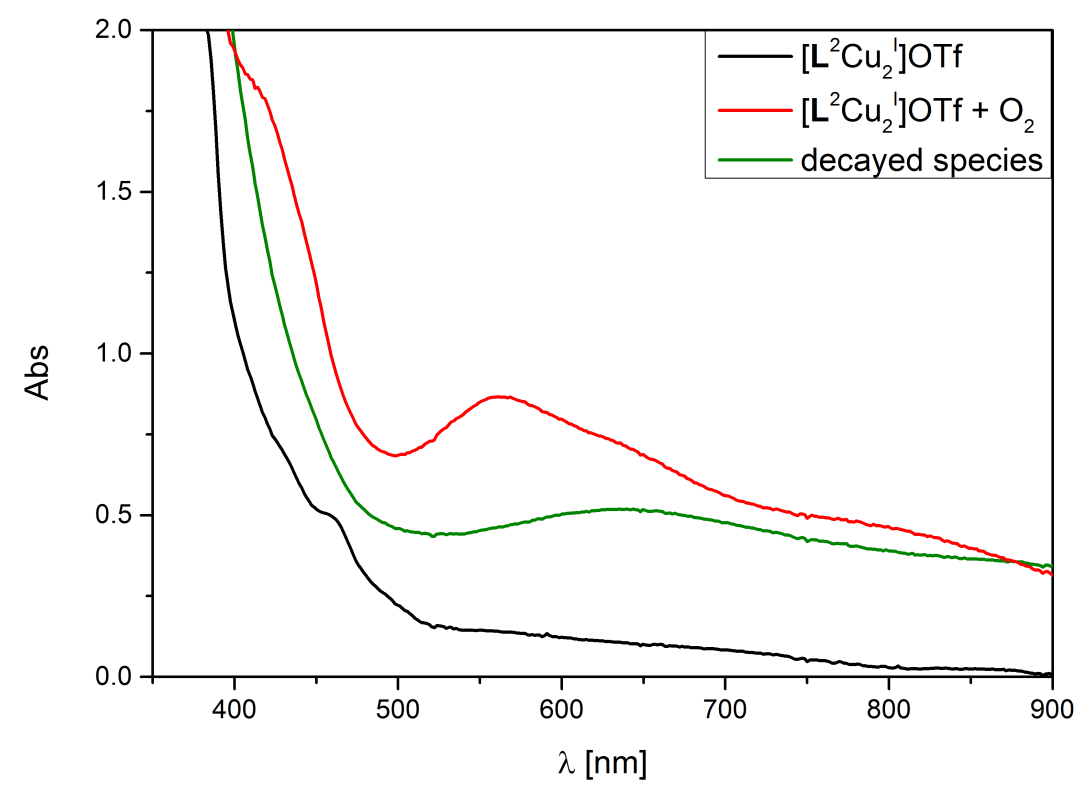

Figure 5.9 UV/Vis spectra of the reaction of $\mathbf{L}^{2} \mathrm{Cu}_{2}^{\mathrm{I}} \mathrm{OTf}$ with dioxygen in EtCN at -80 ${ }^{\circ} \mathrm{C}$.

\subsubsection{Reactivity of $\left[\mathrm{L}^{2} \mathrm{Cu}_{2}^{\mathrm{II}}\left(\mathrm{NO}_{3}\right)_{2}\right]\left(\mathrm{NO}_{3}\right)$ with sodium 2,4,6- trimethyl phenolate}

Lacking the endogenous base present in $\mathbf{1}$, an alternative method to bind a phenolate to the dicopper center was pursued by prior deprotonation of the employed substrate, 2,4,6-trimethyl phenol. The synthesis was carried out by dissolving TMP in dry THF and adding an equimolar amount of sodium hydride. The clear colourless to slightly purple solution was stirred until gas evolution ceased and was subsequently filtered, evaporated to dryness and the product washed with MeCN. However, storage and use of the solid sodium phenolate proved to be challenging, because of its strong hygroscopicity. Upon contact of the white solid with atmospheric water, it turned into a sticky, green-purple 
coloured paste and therefore was stored in a glovebox. NMR spectroscopic investigations indicated a pure compound with 0.5 equivalents of MeCN remaining. Where neccessary, the phenolate was prepared in situ.

\subsubsection{Reactivity of $\left[\mathrm{L}^{2} \mathrm{Cu}_{2}^{\mathrm{II}}\left(\mathrm{NO}_{3}\right)_{2}\right]\left(\mathrm{NO}_{3}\right)$ with one equivalent of NaTMP}

Similar to the reaction of $\mathbf{1}$ with TMP, $\mathbf{4}$ was first tested for its reactivity with one equivalent of NaTMP. In contrast to the reaction with $\mathbf{1}$, no LMCT band was observed in

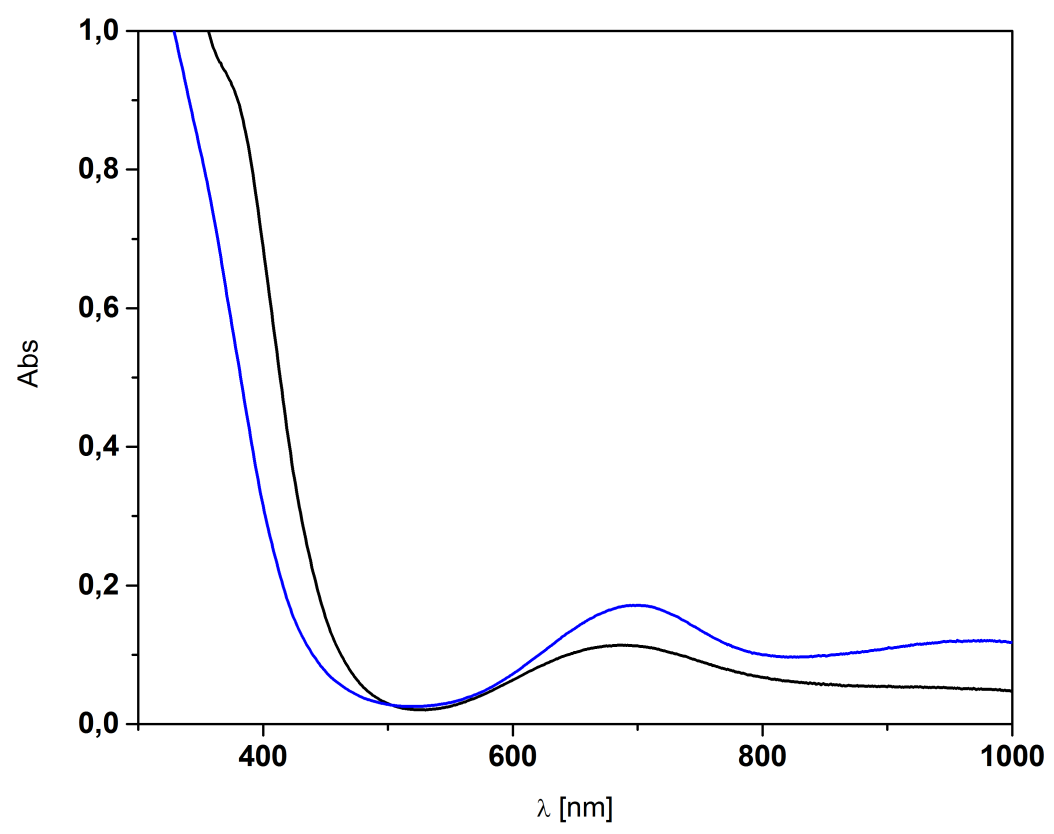

Figure 5.10 UV/Vis spectra of $4\left(c=10^{-3} \mathrm{M}\right)$ (blue) and $4\left(c=10^{-3} \mathrm{M}\right)$ and one equivalent of NaTMP (black) in MeCN.

the UV/Vis spectrum in the region around $495 \mathrm{~nm}$ (Figure 5.10), when one equivalent of NaTMP was added. At $410 \mathrm{~nm}$ a shoulder arises that was assigned to a LMCT transition of the ligand framework. The complex shows an absorption at $700 \mathrm{~nm}$, typical for a orbital forbidden $d$ - $d$ transition. When the reaction mixture is left under anerobic conditions the $d-d$ band is shifted to $685 \mathrm{~nm}$ and its intensity reduced by half, indicating a reduction of half of the copper(II) ions to copper(I) and most likely the presence of a mixed valence species as observed before for the reaction of $\mathbf{1}$ and TMP.

EPR measurement of this solution indeed showed a signal typical for a mononuclear copper(II) complex (Figure 5.11). From the simulation values of $g_{\perp}=2.06(\mathrm{~A}=8 \mathrm{MHz})$ and $\mathrm{g}_{\|}=2.25(\mathrm{~A}=440 \mathrm{MHz})$ were obtained. This result is consistent with the previously observed behaviour of $\mathbf{1}$ and TMP under anaerobic conditions.

The product of the reaction was analyzed by ${ }^{1} \mathrm{H}-\mathrm{NMR}$ and GC-MS analsysis and, just 


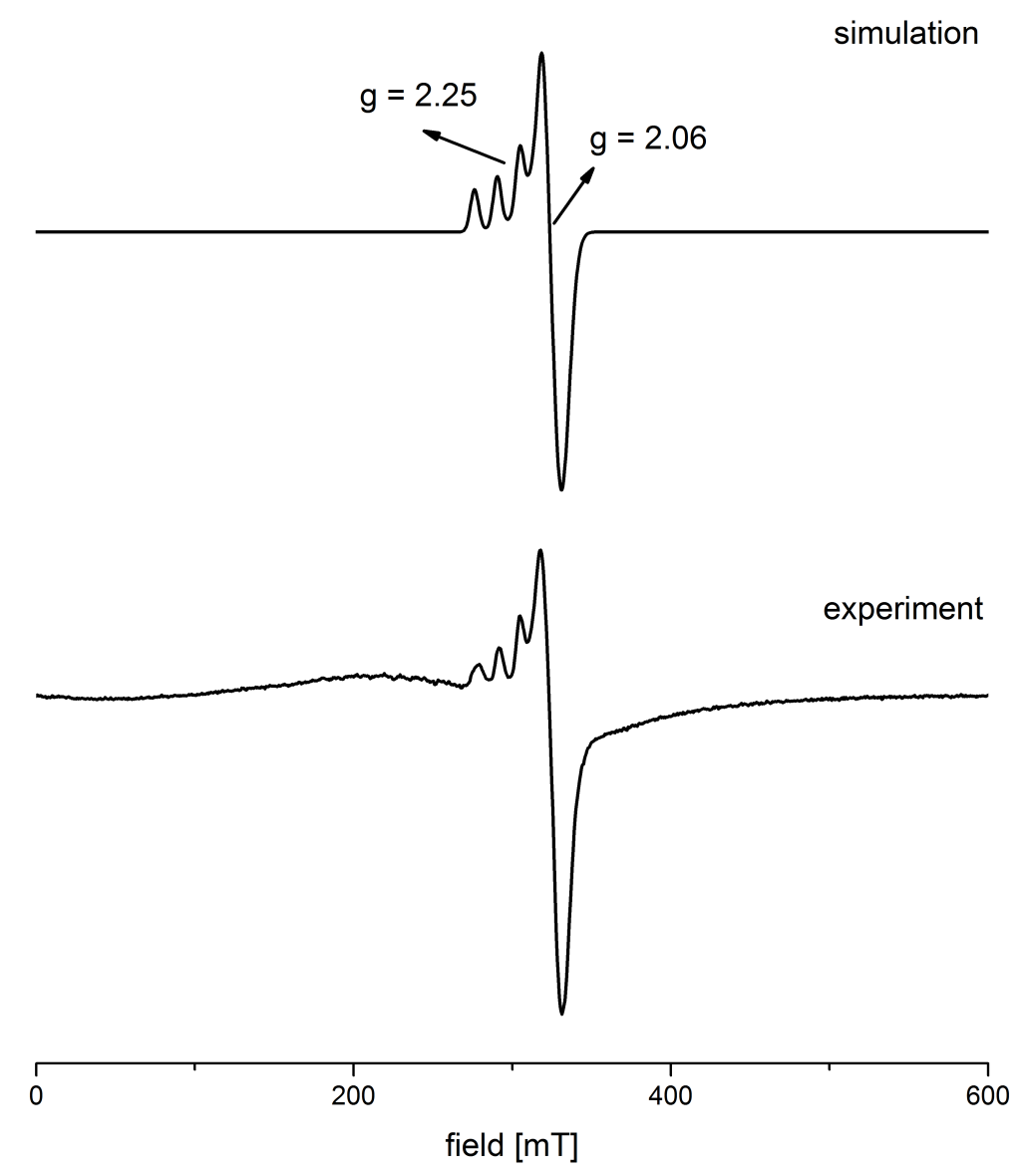

Figure 5.11 EPR spectrum and simulated spectrum of a solution containing 4 and one equivalent of NaTMP in MeCN at $160 \mathrm{~K}$ (simulation parameters: nuleus $=\mathrm{Cu}$, total spin $=1 / 2$, linewidth $=8 \mathrm{mT}$, frequency $=9.430 \mathrm{GHz}, \mathrm{g}_{\|}=2.25, \mathrm{~g}_{\perp}=2.06, \mathrm{~A}_{\|}=0.0142 \mathrm{~cm}^{-1}$, $\mathrm{A}_{\perp}=0.0013 \mathrm{~cm}^{-1}$, gstrain $\left.=[0.05,0.1,0.05]\right)$.

as in the case of $\mathbf{1}$, was found to be TMBB under anerobic and TMSQ under aerobic conditions.

\section{Reactivity of $\left[\mathrm{L}^{2} \mathrm{Cu}_{2}^{\mathrm{II}}\left(\mathrm{NO}_{3}\right)_{2}\right]\left(\mathrm{NO}_{3}\right)$ with two equivalents of $\mathrm{NaTMP}$}

When two equivalents of base were added to the reaction of TMP and $\mathbf{1}$, the reaction pathway changed and the reaction afforded 4-hydroxy-3,5-dimethyl benzaldehyde (HDB) as the only product. In this section, the reaction of $\mathbf{4}$ and two equivalents of NaTMP will be investigated.

Upon addition of the second equivalent NaTMP, a LMCT band arose at $480 \mathrm{~nm}$ Figure 5.12). Under anaerobic conditions, this band as well as the $d-d$ band decreases within a few minutes at room temperature, along with the appearace of a small sharp band at $580 \mathrm{~nm}$. This band most likely originates from the complex. Filtration of the solution through silica, which only leaves organic compounds, resulted in a disappearance 
of the band.

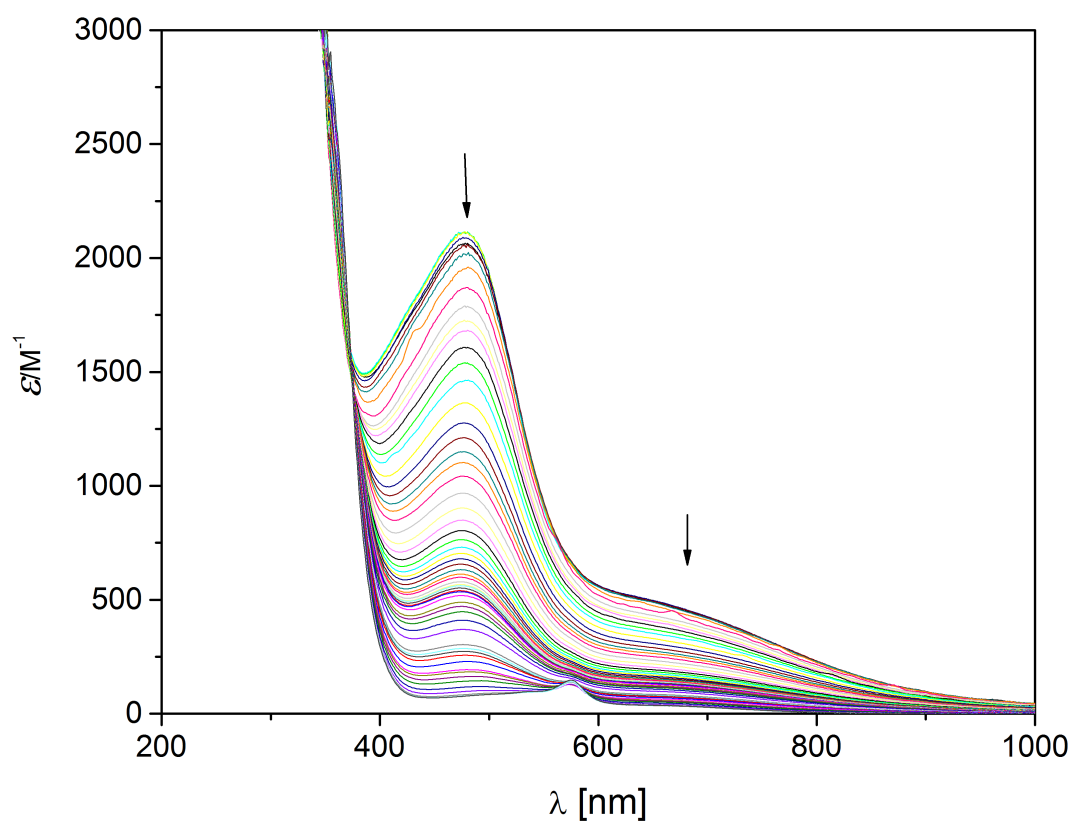

Figure 5.12 UV/Vis spectra of a solution of $4\left(\mathrm{MeCN}, c=10^{-3} \mathrm{M}\right)$ and two equivalents of NaTMP warmed from $-20^{\circ} \mathrm{C}$ to $+20^{\circ} \mathrm{C}$.

The red coloured MeCN solution obtained after adding two equivalents of NaTMP was immediately frozen in liquid nitrogen to prevent a reaction and a resonance Raman spectrum was recorded at $77 \mathrm{~K}$ (Figure 5.13). The spectrum is very similar to that of the adduct of $\mathbf{1}$ and TMP. The $\mathrm{C}-\mathrm{O}$ stretch $\left(\nu_{7}\right)$ was assigned to the band at $1317 \mathrm{~cm}^{-1}$ and the $\mathrm{C}_{\text {orthto }}-\mathrm{C}_{\text {meta }}$ stretch $\left(\nu_{8}\right)$ to the band at $1610 \mathrm{~cm}^{-1}$. Although the $\nu_{7}$ frequency at $1317 \mathrm{~cm}^{-1}$ is a bit higher in energy compared to the adduct of TMP and $\mathbf{1}$, observed at $1257 \mathrm{~cm}^{-1}$, it still lies in the region of a copper bound phenoxide. $\frac{157}{15}$ Further resonance enhanced features were observed at $1044 \mathrm{~cm}^{-1}, 1559 \mathrm{~cm}^{-1}, 1557 \mathrm{~cm}^{-1}$ and $1572 \mathrm{~cm}^{-1}$.

After the UV/Vis features vanished under anaerobic conditions, another EPR spectrum of the solution was measured. The observed spectrum (Figure 5.14) indicates the complete reduction of the complex to a dicopper(I) state, but still shows some weak signals, the sharp signal likely originates from an organic radical due to its g-value of 2.00. When the reaction mixture is exposed to dioxygen, the LMCT band reappears, along with a strong increase in the sharp band at $580 \mathrm{~nm}$ (Figure 5.15). The same band at $580 \mathrm{~nm}$ was observed, when 1, TMP and a second equivalent of base was reacted in MeCN. Its origin remains unknown.

After the reaction was worked up by filtration through silica, the product was found to be 4-hydroxy-3,5-dimethylbenzaldehyde (HDB), as observed for the previous reaction 


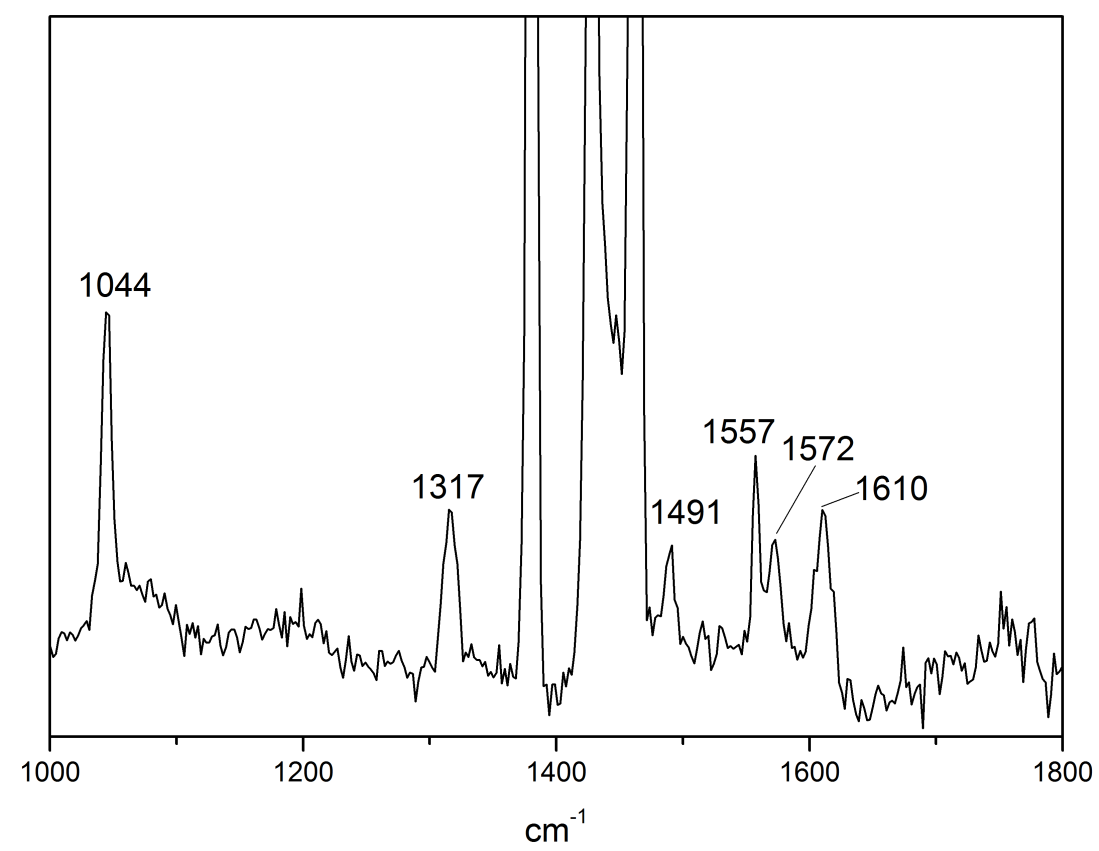

Figure 5.13 Resonance Raman spectrum of 4 and two equivalents of NaTMP in frozen $\mathrm{MeCN}$.

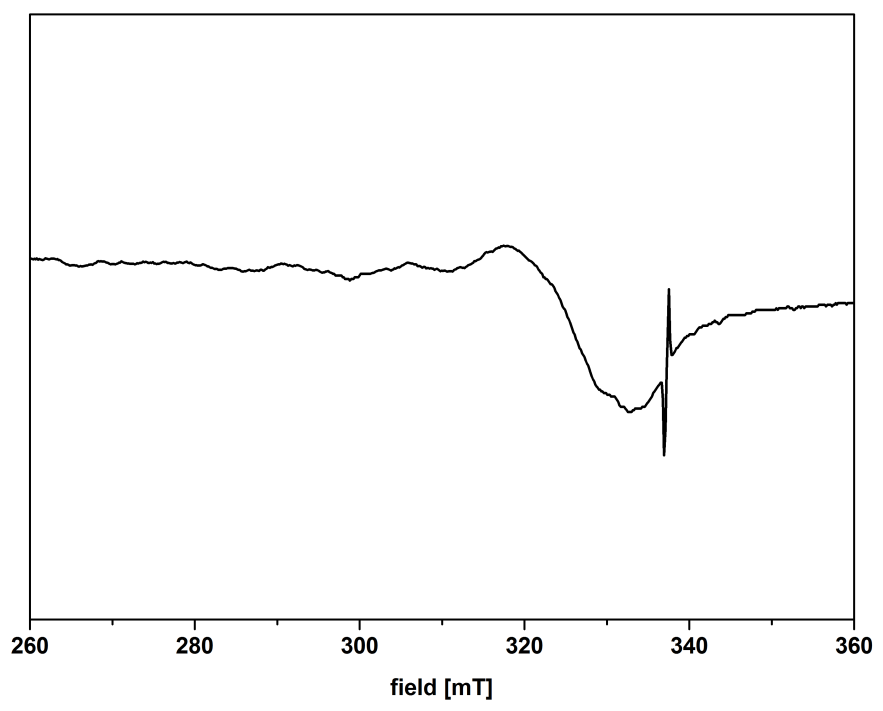

Figure 5.14 EPR spectrum of 4 after the reaction with 2 equivalents of NaTMP in $\mathrm{MeCN}$ at $160 \mathrm{~K}$ under anaerobic conditions.

with 1 . When the reaction is carried out under aerobic conditions, a yield of up to $95 \%$ HDB is possible with $3 \mathrm{~mol} \%$ of $\mathbf{4}$ in MeCN. Carrying out the reaction under an ${ }^{18} \mathrm{O}_{2}$ atmosphere, $100 \%$ incorporation of ${ }^{18} \mathrm{O}_{2}$ was observed.

Reoxidation of the dicopper(I) species

After the origin of the incorporated oxygen was determined, an oxygen uptake experiment in a sealed vessel containing $1.86 \mu \mathrm{mol}$ of $\mathbf{4}$ and $3.70 \mu \mathrm{mol}$ of NaTMP dissolved in 2 


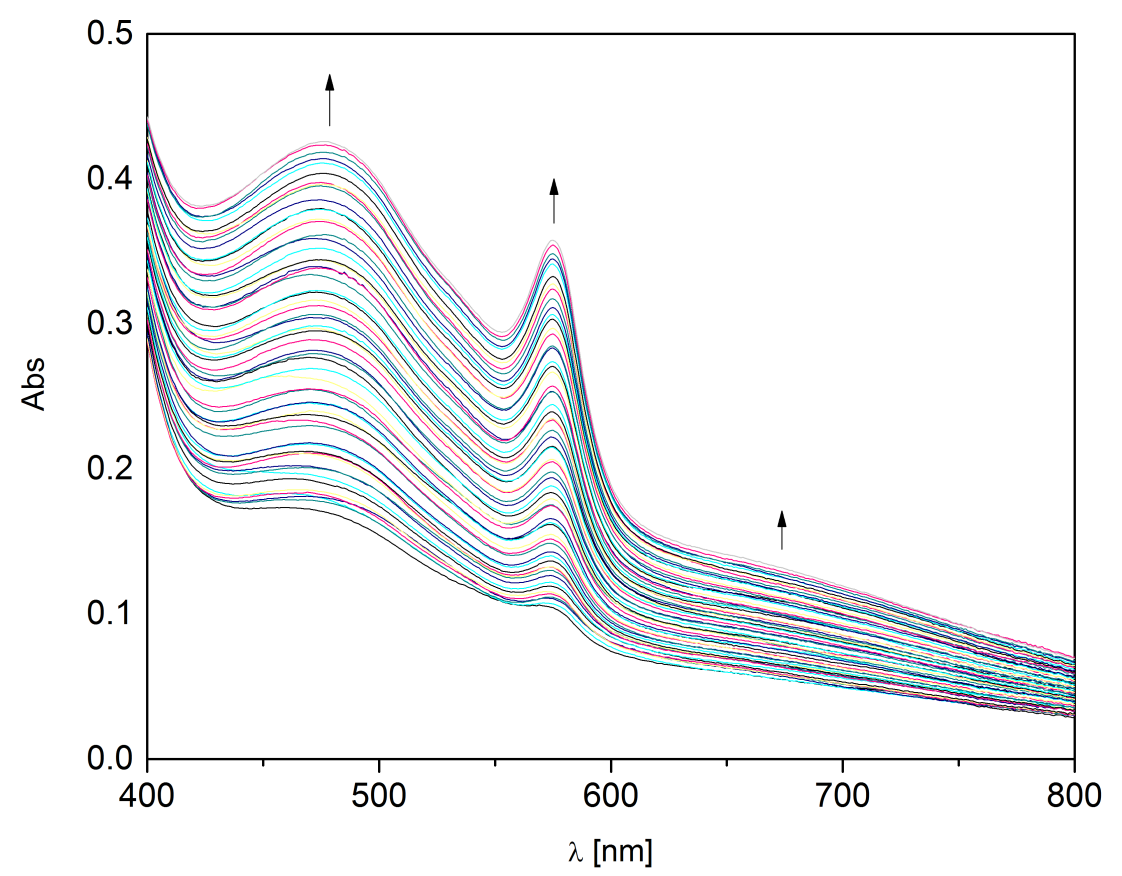

Figure 5.15 UV/Vis spectra after dioxygen exposure of the reaction of 4 with two equivalents of NaTMP in MeCN.

$\mathrm{mL}$ MeCN was performed with a CLARK-electrode setup. The resulting time trace is

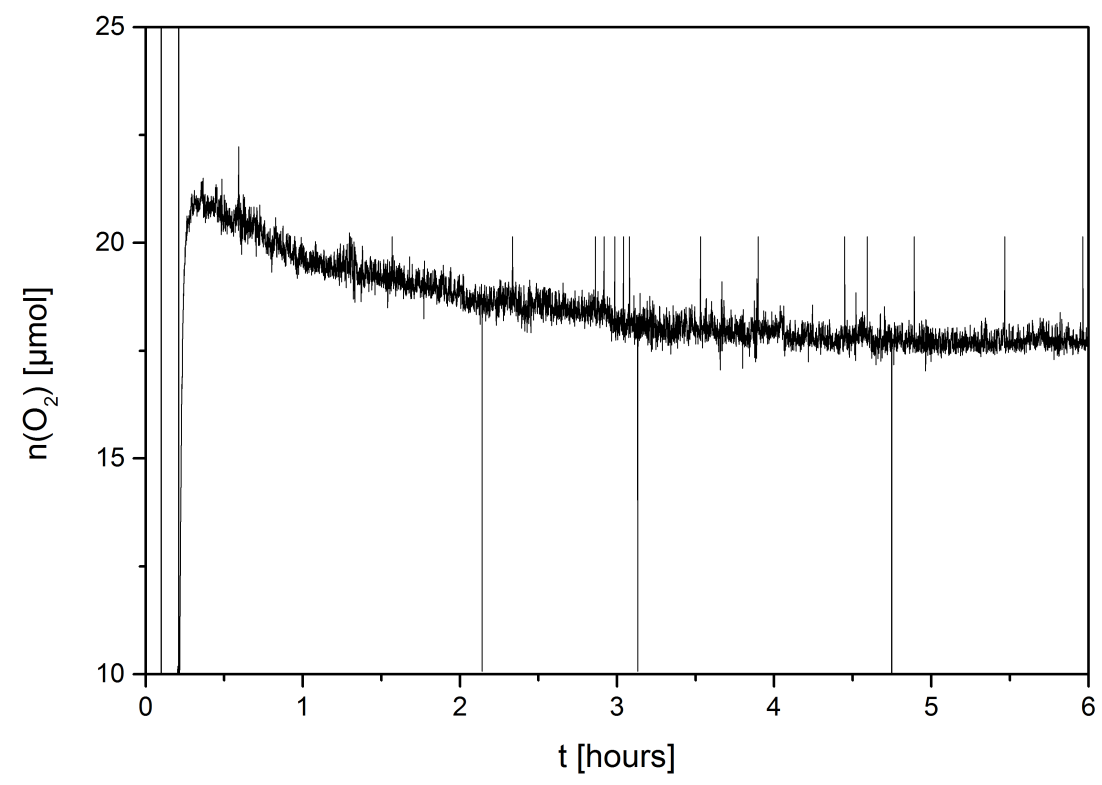

Figure 5.16 Time trace of dioxygen conten in a sealed reaction vessel as determined by a CLARK-electrode.

depicted in Figure 5.16. The reaction was finished after 5 hours and the oxygen content reached a plateau after $3.4 \mu \mathrm{mol}$ of dioxygen were consumed. This suggests a one-to-one stoichiometric ratio of TMP $/ \mathrm{O}_{2}$. One molecule of $\mathrm{O}_{2}$ can take up up to four electrons, which means one atom can take up two electrons. One oxygen atom is incorporated into the product and reduced to a -II state and the other atom is needed to reoxidize the 
dicopper(II) complex. Therefore, the second oxygen atom is reduced to water, with the two neccessary protons coming from the substrate's para- $\mathrm{CH}_{3}$ group. One of the two is abstracted after oxygen incorporation and the other is recovered from the base (NaTMP) to enable another catalytic cycle.

In conclusion, the reactivity of the catalyst with TMP and two equivalents of base are the same in the case of $\mathbf{4}$ and $\mathbf{1}$. Both give 4-hydroxy-3,5-dimethylbenzaldehyde as the only product and in both cases the source of the incorporated oxygen is aerial dioxygen. An experiment with $\mathbf{1}$ and two equivalents of NaTMP also gave HDB as the only product. Similar results were observed for the reaction of $\mathbf{4}$ with TMP and two equivalents of DBU.

A control experiment with $\mathrm{CuCl}_{2}$ and different bases in methanol always yielded complicated mixtures of products, including TMBB, TMSQ, MDB, the benzaldehyde and other non-identified compounds.

\section{Kinetic investigations}

To gain insight into the mechanism of this reaction, the decay of the LMCT band was monitored over time and evaluated using the method of reaction progress kinetic analysis. Figure $5.17 \mathrm{~b}$ shows the decay of the LMCT band over time. The decay was

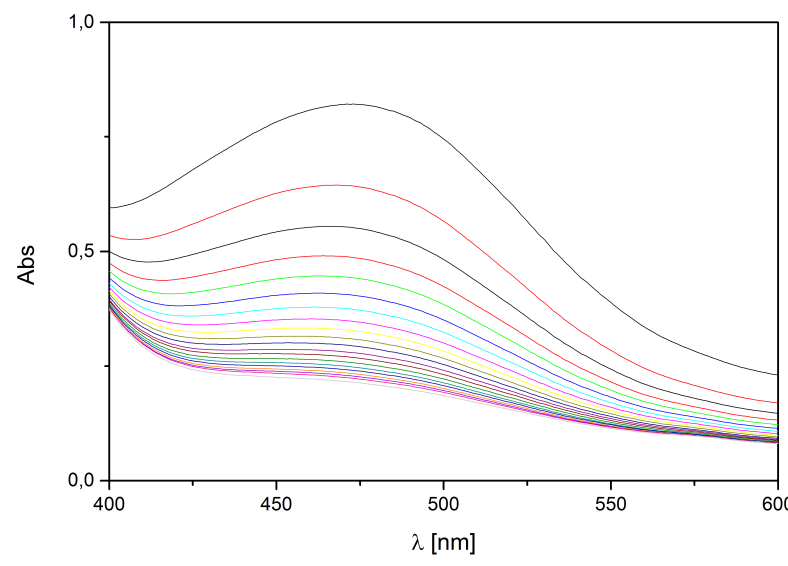

(a) $\mathrm{UV} / \mathrm{V}$ is spectra of a solution of $\mathbf{4}(c=$ $10^{-3} \mathrm{M}$ ) and 2 equivalents of NaTMP at $0{ }^{\circ} \mathrm{C}$. A spectrum was recorded every two minutes.

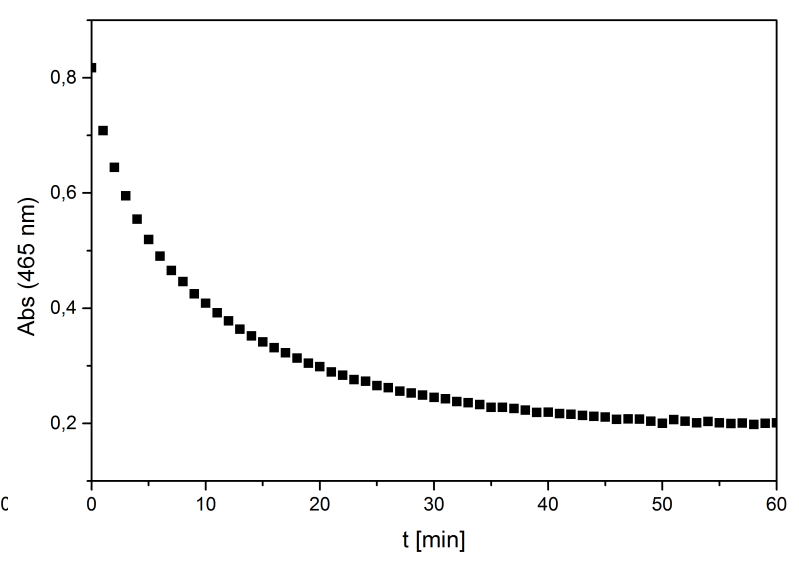

(b) Time trace of the absorption at $465 \mathrm{~nm}$.

Figure 5.17 UV/Vis spectra of the decay of the LMCT band and time trace at $465 \mathrm{~nm}$.

fitted exponentially and the obtained values used to calculate the derivative that was plotted against time (5.18b). In order to obtain the reaction order, the reaction rate is plotted against the momentary concentration of the intermediate. The slope represents the $\mathrm{k}$-value and the concentration of the intermediate (assumed to be the adduct of phenol and catalyst $[\mathrm{P}-\mathrm{S}])$ is factored in corresponding to its reaction order (termed a): 


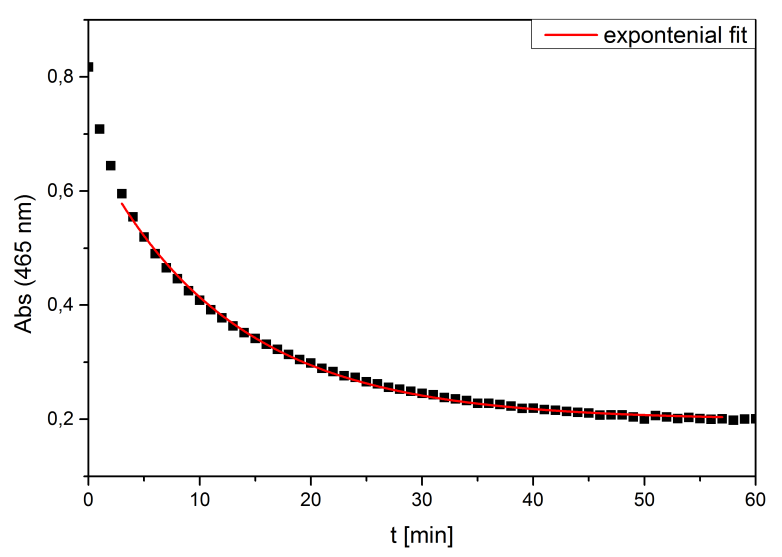

(a) time trace of the reaction at $465 \mathrm{~nm}$ (black squares) and exponential decay fit (red line)

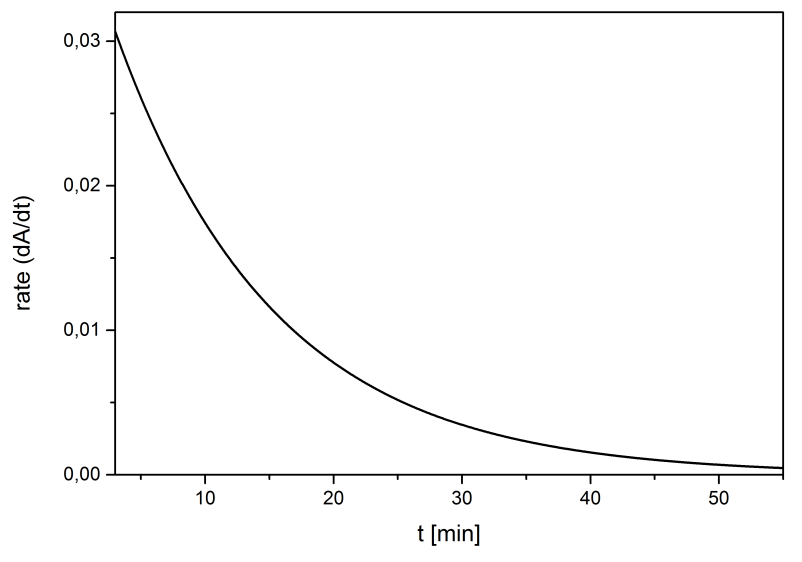

(b) reaction rate $(\mathrm{dA} / \mathrm{dt})$ plotted against time

Figure 5.18 Kinetic evaluation of the decay of the LMCT band at $465 \mathrm{~nm}$.

$$
y=k \cdot[\mathrm{P}-\mathrm{S}]^{a}
$$

The plot yields a straight line (Figure 5.19), which indicates a first order kinetic, because the rate exhibits a linear dependence on concentration of the intermediate. The $k$-value and was determined to be $0.081 \mathrm{~min}^{-1}$.

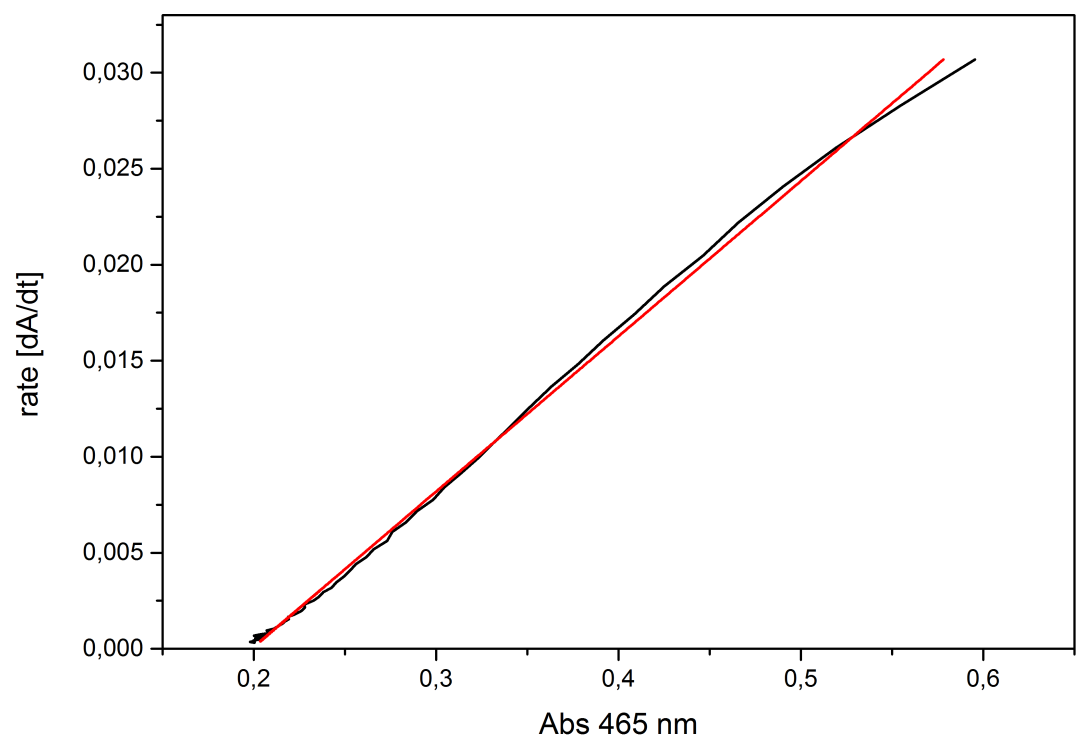

Figure 5.19 black line) reaction rate of collected data versus momentary absorption. red line) fitted reaction rate vs momentary absorption

The same process was used for the reaction with TMP-d $\mathrm{d}^{9}$ Figure 5.20. As expected the reaction is also first order for TMP- $\mathrm{d}^{9}$. The rate constant $\mathrm{k}_{\mathrm{obs}}$ was determined to be $0.0259 \mathrm{~min}^{-1}$. This results in a kinetic isotope effect (KIE) of $\mathrm{k}_{\mathrm{H} / \mathrm{D}}=3.1$, which indicates a primary KIE with direct involvement of the $\mathrm{C}-\mathrm{H} / \mathrm{D}$ bond. Secondary kinetic isotope effects on the pKa of the $\mathrm{O}-\mathrm{H}$ bond in similar compounds were found to be 


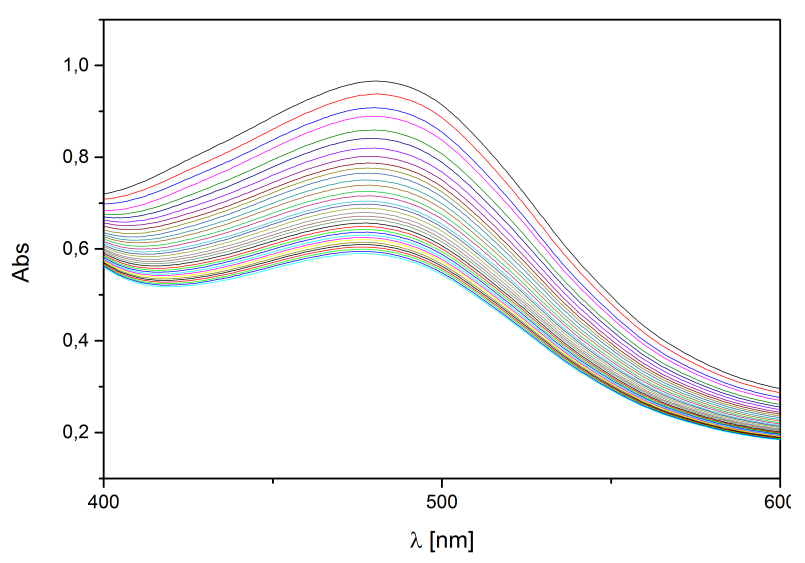

(a) $\mathrm{UV} / \mathrm{V}$ is spetra of a solution containing 4 $\left(\mathrm{c}=10^{-3} \mathrm{M}\right.$ and 2 equivalents of NaTMP- $\mathrm{d}^{9}$ at

$0{ }^{\circ} \mathrm{C}$. One spectrum every two minutes.

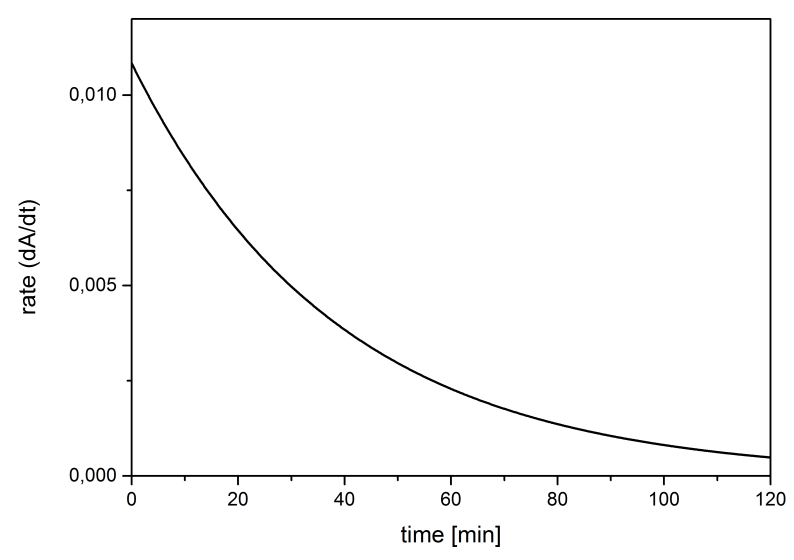

(c) reaction rate plotted against time

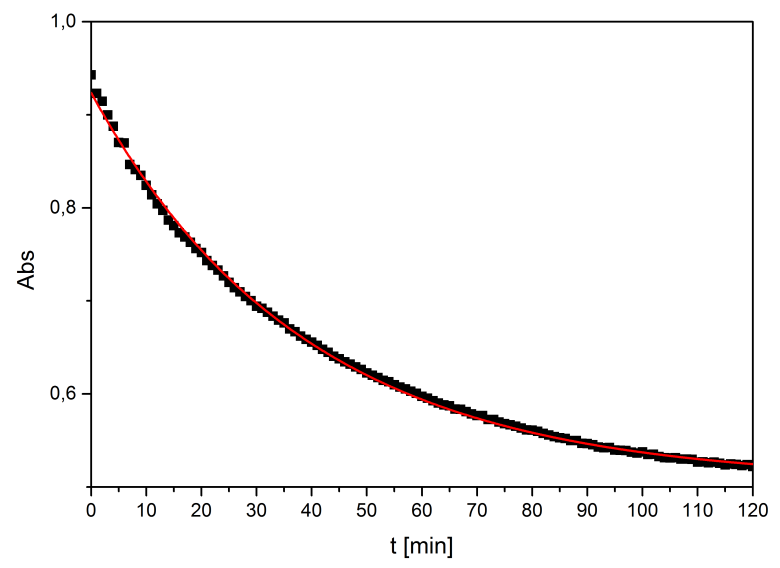

(b) time trace of the absorption at $465 \mathrm{~nm}$ (black squares) and exponential decay fit (redline)

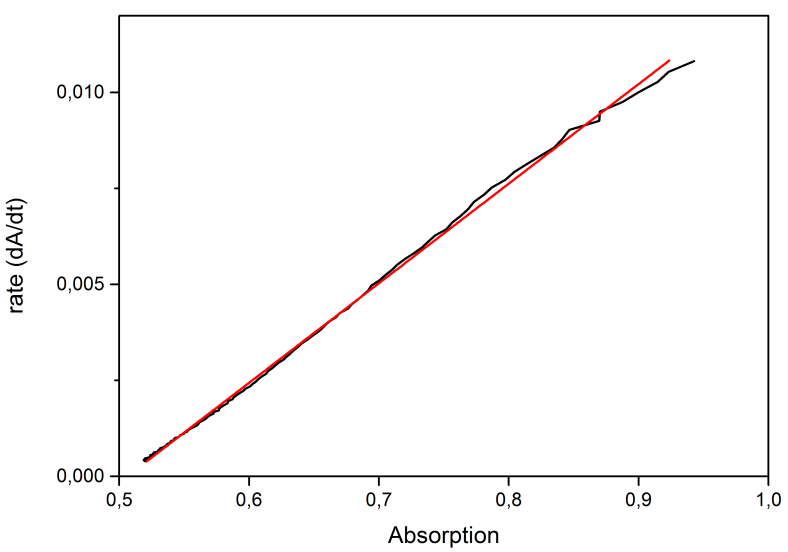

(d) black line) reaction rate of collected data versus momentary absorption. red line) fitted reaction rate vs momentary absorption

Figure 5.20 Evaluation of the reaction progress kinetics.

around 1.02 to 1.05. 194 A KIE of of 5.6 was observed for the para-C-H deprotonation in a tautomerization reaction of an ortho-quinone to the corresponding para-quinone methide. $\frac{195}{}$ A kinetic isotope effect of hybridization from $\mathrm{sp}^{3}$ to $\mathrm{sp}^{2}$ lies in the range of 1.2 to 1.4 . $196[197$

An interpretation of the witnessed KIE of 3.1 is difficult, because there is no data available for a direct comparison. Nevertheless, it points towards a primary KIE in the formation of a quinone methide, when compared to the mentioned tautomerization. 


\subsection{Nature of the intermediate}

For reactions that involve a two-electron oxidation of ortho- or para-substituted phenols a quinone methide intermediate has been proposed. Quinone methides are highly polarized compounds that are difficult to observe and sometimes only proposed as

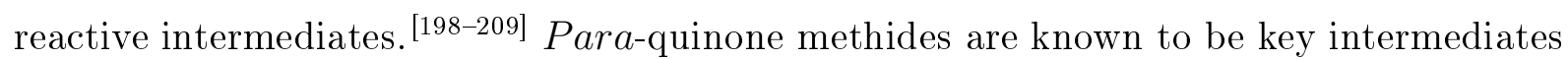
in the biosynthesis of natural polymers lignin and melanin. $\frac{210}{20}$ They owe their instability to their zwitterionic nature (Figure 5.21).

Several groups have succeeded in stabilizing these reactive compounds through transition

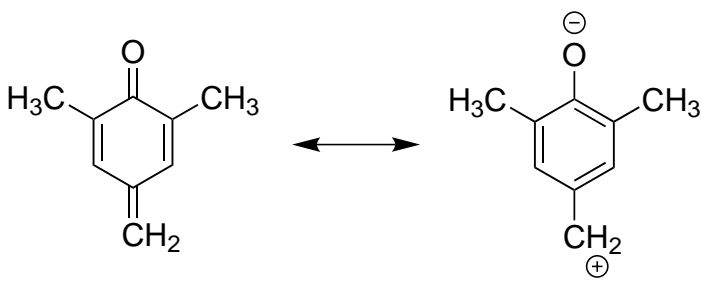

Figure 5.21 Representation of the zwitterionic nature of para-quinone methides.

metal coordination. 211-215 Due to their reactive nature, characterization of these intermediates is difficult and only few reference NMR and UV/Vis-spectra exist in the literature. 216-219]

As mentioned before, they are often characterized by their reactivity. Ortho-quinone methides serve as synthetic precoursers with a reactivity similar to $\alpha, \beta$-unsaturated ketones. 220 Their reactivity includes a variety of cycloadditions like the inter- and intramolecular Diels-Alder reaction, 221222$][4+1]$ cyclo additions, 201$]$ nucleophilic addition to the methide carbon 201 and rearrangement reactions. ${ }^{220}$

Para-quinone methides act as Michael acceptors and undergo 1,6-conjugate addition or annulation. $223+225$

While there have been reports of the direct synthesis of aldehdes from methyl substituted phenols, and some propose a quinone methide intermediate, ${ }^{226}$ there are no studies that further elucidate the mechanism of the reaction. Other working groups were monitoring the amount of quinone methide in their reaction mixture by means of mass spectrometry coupled to GC analysis. 2031227

A first attempt to grasp the nature of the intermediate was made by ESI mass spectrometry. The reaction mixture of $\mathbf{1}, \mathrm{TMP}$ and $\mathrm{DBU}$ as well as the reaction mixture of $\mathbf{4}$ and two equivalents of NaTMP were analyzed to detect possible quinone methide intermediates. A spectrum measured by GHosH is depicted in Figure 5.22, A possible 2,6-dimethyl-quinone methide intermediate bound to the dicopper complex, $\left[\mathrm{L}^{2} \mathrm{Cu}_{2}\left(\mathrm{OC}_{9} \mathrm{H}_{10}\right)\right]^{+}$, was found at $m / z=1017.4$ though the peak intensity is very low. 


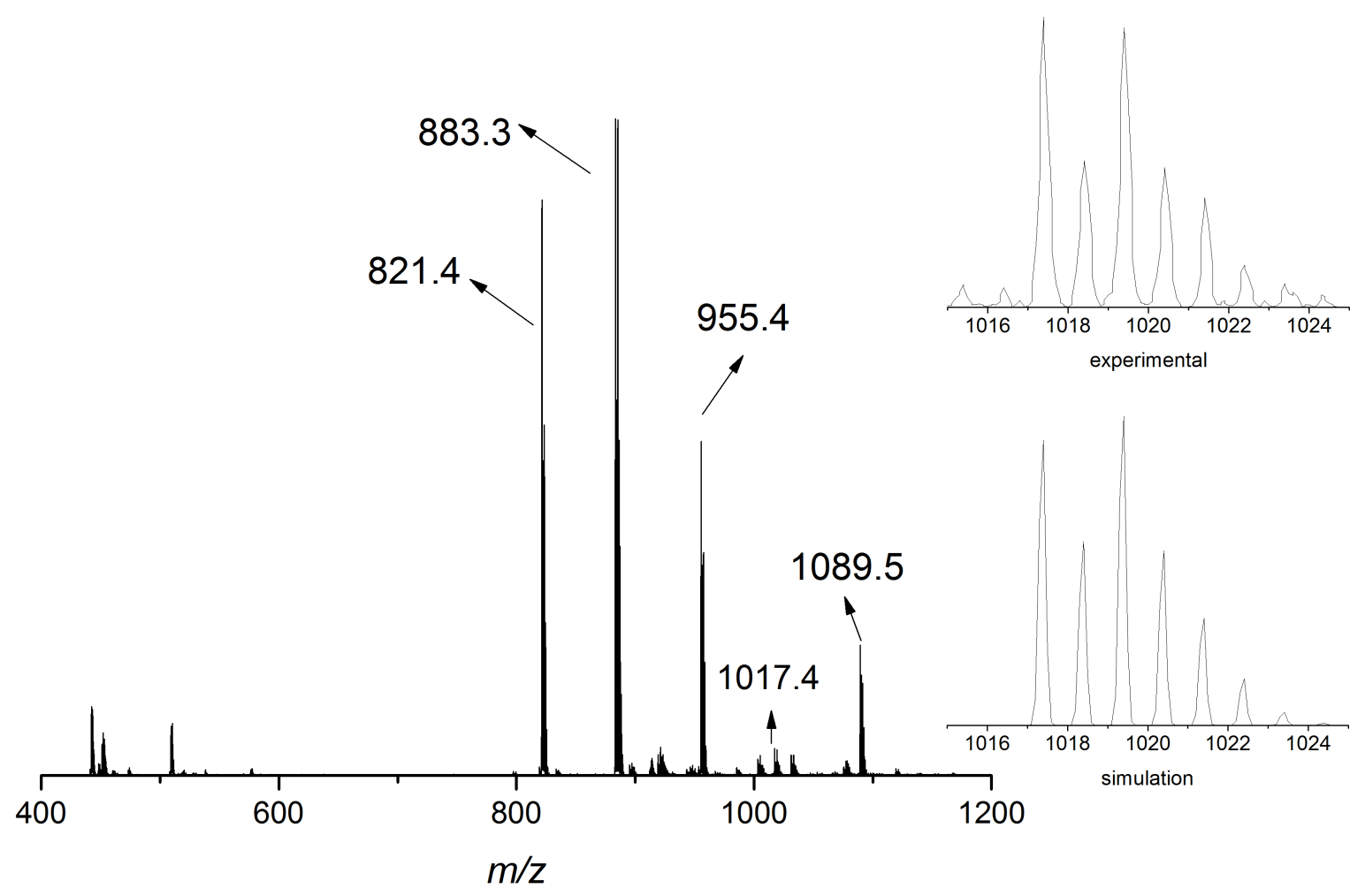

Figure 5.22 ESI mass spectrum of the reaction solution of 4 and 2 equivalents of NaTMP in MeCN measured under inert conditions. Inset shows enlargemnt and simulation of a postulated quinone methide bound to the dinuclear copper(I) complex $\left(\left[\mathbf{L}^{2} \mathrm{Cu}_{2}\left(\mathrm{OC}_{9} \mathrm{H}_{10}\right)\right]^{+}\right)$.

The low intensity of the peak for this intermediate can be explained either by the rapid dissociation of the generated methide from the reduced metal complex or its high reactivity. The main peak of the spectrum corresponds to the dicopper(I) complex found at $m / z=883.3\left(\left[\mathbf{L}^{2} \mathrm{Cu}_{2}\right]^{+}\right)$. The second most intense peak at $m / z=821.4$ corresponds to a demetallated monocopper $(\mathrm{I})$ species $\left(\left[\mathrm{L}^{2} \mathrm{Cu}+\mathrm{H}\right]^{+}\right)$that is always observed for the dicopper(I) species and most likely is a result of the ionisation in the spectrometer. A peak at $m / z=955.4$ was assigned to a protonated monocopper(I) species, with a bound quinone methide $\left[\mathrm{L}^{2} \mathrm{Cu}\left(\mathrm{OC}_{9} \mathrm{H}_{10}\right)\right]^{+}$. Due to its charge, no other species can be assigned to this peak. The peak at $m / z=1089.5$ could not be unambigiuosly identified but fits to a simulation of the protonated mononuclear copper(I) complex with a para-C-O coupled diphenoquinone methide.

An ESI mass spectrum of the reaction mixture of $\mathbf{1}$, TMP and DBU in MeCN is depicted in Figure 5.23. At $m / z=761.2$ an analogous species that was assigned to the quinone methide bound to the dicopper(I) complex was found $\left(\left(\left[\mathbf{L}^{1} \mathrm{Cu}_{2}\left(\mathrm{OC}_{9} \mathrm{H}_{10}\right)\right]^{+}\right)\right.$. A protonated species with one copper less was found at $m / z=699.3\left(\left[\mathbf{L}^{1} \mathrm{Cu}\left(\mathrm{OC}_{9} \mathrm{H}_{10}\right) \mathrm{H}\right]^{+}\right)$. Futhermore the dicopper(I) species $\left(\left[\mathbf{L}^{1} \mathrm{Cu}_{2}\right]^{+}\right)$was found at $m / z=627.2$ and the 


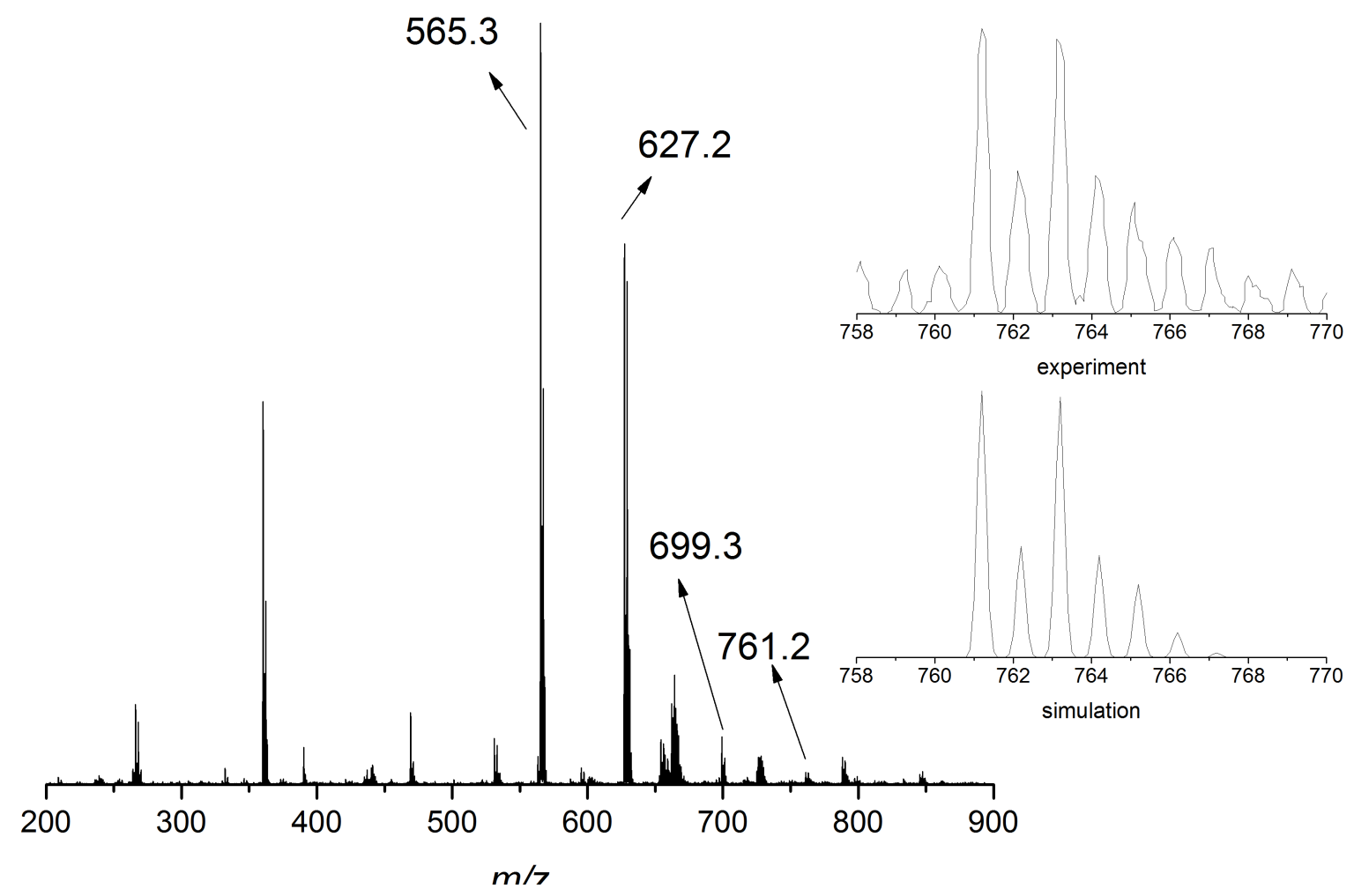

Figure 5.23 ESI mass spectrum of the reaction solution of 1 and 2 equivalents of NaTMP in MeCN measured under inert conditions. Inset shows enlargemnt and simulation of a postulated quinone methide bound to the dinucler copper(I) complex $\left(\left[\mathbf{L}^{1} \mathrm{Cu}_{2}\left(\mathrm{OC}_{9} \mathrm{H}_{10}\right)\right]^{+}\right)$.

protonated moncopper(I) species $\left(\left[\mathbf{L}^{1} \mathbf{C u}+\mathrm{H}\right]^{+}\right)$at $m / z=$ at 565.3 as the major peak. In conclusion, in ESI mass spectra, peaks were found that fit to a possible quinone methide intermedate. Because of the small difference of $m / z=1$ between a phenolate and a quinone methide bound to the respective complex, further investigation is neccessary to clarify the nature of the intermediate.

To characterize the intermediate further, NMR spectra of the reaction mixtures under inert conditions and independently generated quinone methides were recorded. Because reference data was only available in $\mathrm{CCl}_{4}$ as solvent, $\mathrm{CDCl}_{3}$ was chosen to first characterize the para-quinone methide gerenated from 2,4,6-trimethyl phenol. it was synthesized in situ with $\mathrm{Ag}_{2} \mathrm{O}$ in chloroform. 2181219] The reaction was carried out in $\mathrm{CDCl}_{3}$ with a substoichiometric amount to prevent overoxidation. The resulting spectrum is shown in Figure 5.24.

Peaks that belong to TMP are denoted as such along with the chemical shift in the spectrum and are found at $2.22 \mathrm{ppm}, 4.45 \mathrm{ppm}$ and $6.79 \mathrm{ppm}$. New signals are found at $1.58,2.01,5.75$ and $6.99 \mathrm{ppm}$. There are two other possible oxidation products of 


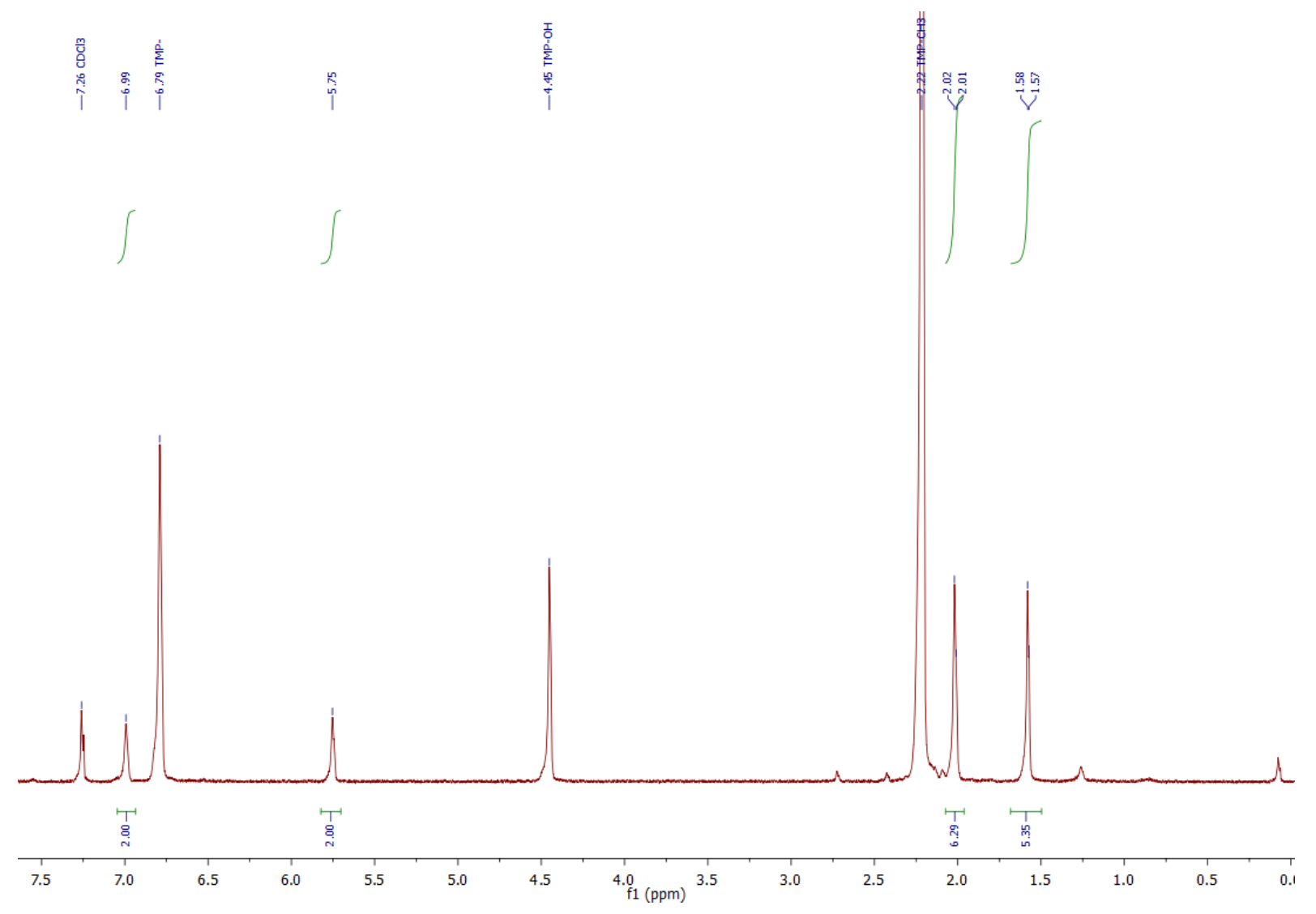

Figure $5.24{ }^{1} \mathrm{H}-\mathrm{NMR}$ spectrum of the reaction mixture containing TMP and $\mathrm{Ag}_{2} \mathrm{O}$ in $\mathrm{CDCl}_{3}$

this reaction. Radical reactions and decay of the quinone methide intermediate lead to the formation of TMBB and TMSQ. However, their NMR signals are found at different shifts than the newly found peaks and are therefore not present in the solution. While the peak at $1.58 \mathrm{ppm}$ coud not be explained, the remaining signals fit well to a possible quinone methide. The peak found at $2.01 \mathrm{ppm}$ corresponds to the ortho- $\mathrm{CH}_{3}$ groups with an integral of 6 . A peak found at $5.57 \mathrm{ppm}$ with an integal of 2.00 fits well to the methide $\mathrm{CH}_{2}$ group. The aromatic protons with an integral of 2.00 could be found at $6.99 \mathrm{ppm}$. Literature values for the reaction in $\mathrm{CCl}_{4}$ are $1.90 \mathrm{ppm}$ for the $\mathrm{CH}_{3}$ groups, $5.49 \mathrm{ppm}$ for the methide protons and $6.76 \mathrm{ppm}$ for the aromatic protons and are in good agreement with the values obtained in $\mathrm{CDCl}_{3} .{ }^{218}$ These shifts will be used as a reference for comparing NMR data with those of the reaction solutions that will be investigated.

When the reaction is carried out in $\mathrm{CD}_{3} \mathrm{CN}$, shifts are a little different compared to $\mathrm{CDCl}_{3}$ but assignment of the signals was possible nevertheless. A NMR spectrum of the reaction of TMP and $\mathrm{Ag}_{2} \mathrm{O}$ in $\mathrm{CD}_{3} \mathrm{CN}$ is depicted in Figure 5.25. Signals for TMP are found at 2.14, 2.16, 5.81 and $6.74 \mathrm{ppm}$. Traces of TMBB are also present. Its signals are found at 2.67 and $6.79 \mathrm{ppm}$. The remaining expected signals for TMBB at 2.12, 2.15 


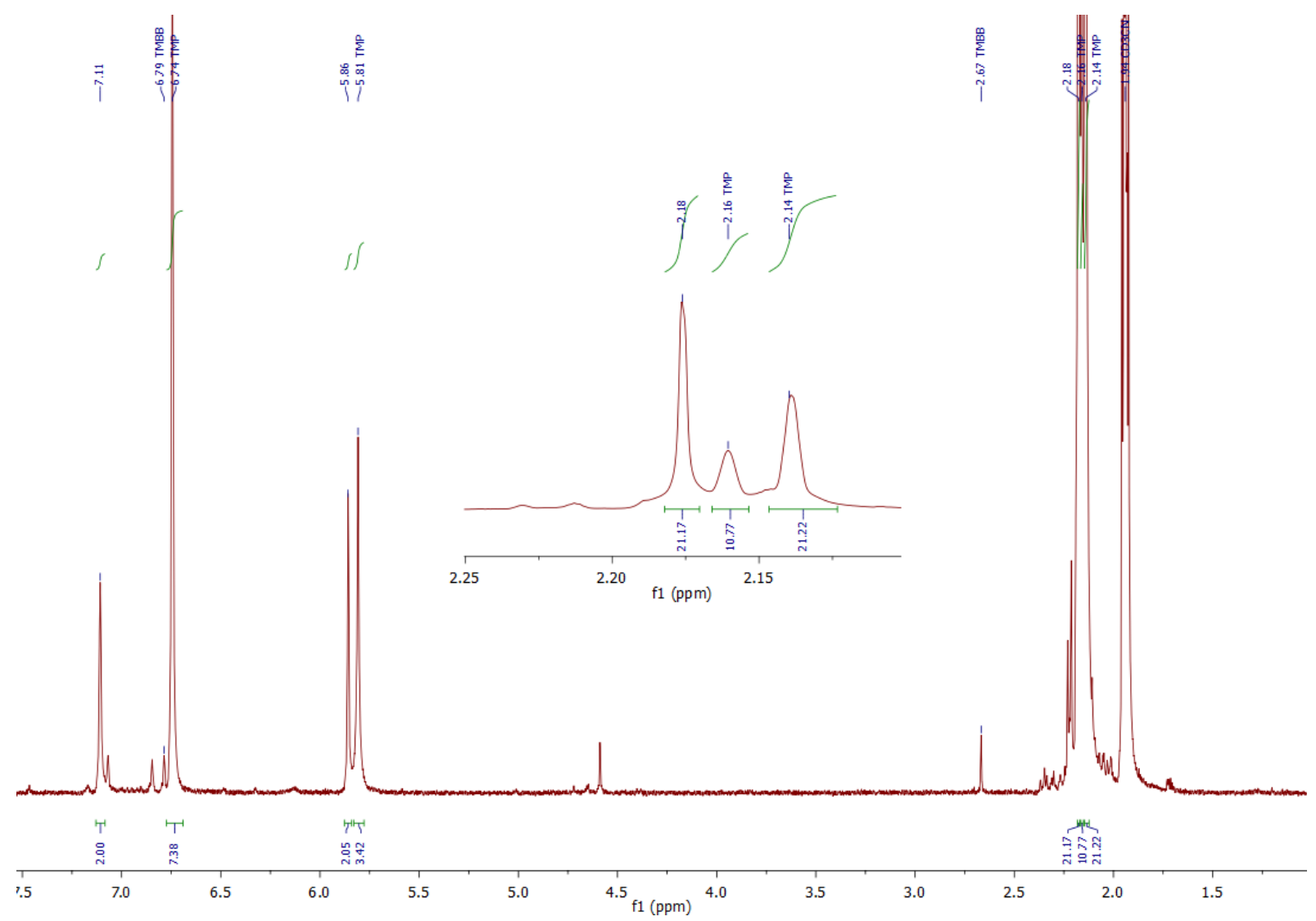

Figure $5.25{ }^{1} \mathrm{H}-\mathrm{NMR}$ spectrum of the reaction mixture containing TMP and $\mathrm{Ag}_{2} \mathrm{O}$ in $\mathrm{CD}_{3} \mathrm{CN}$. Inset shows an enlargement of the area around $2.15 \mathrm{ppm}$.

and $6.79 \mathrm{ppm}$ are not visible due to an overlap with stronger signals of different origin. Signals found at 2.18, 5.86 and $7.11 \mathrm{ppm}$ were assigned to the $p$-quinone methide species. The $\mathrm{CH}_{3}$ group at $2.18 \mathrm{ppm}$ has an unusually high integral and probably overlaps with another signal. The signal at 5.86 with an integral of 2 falls within the range of the methide protons. A peak at $7.11 \mathrm{ppm}$ for the aromatic protons with an integral of 2 was assigned to the protons in meta position of the ring. All those signal are in the same range as those observed in $\mathrm{CDCl}_{3}$ and $\mathrm{CCl}_{4}$ and will be used as reference.

A ${ }^{1} \mathrm{H}-\mathrm{NMR}$ spectrum of the reaction mixture of $\mathbf{4}$ and two equivalents of NaTMP, measured by GHosh, is depicted in Figure 5.26. Comparing the assigned methide peak to the pyrazole proton in the dicopper(I) complex shows an almost identical shift for both protons, which could make assignment difficult.

Assignment of the complex and TMP signals was achieved by comparison with previous spectra. There are two new signals in the aromatic region at 6.83 and $7.04 \mathrm{ppm}$ and three new signals in the region of the $\mathrm{CH}_{3}$ groups at 2.17, 2.19 and $2.22 \mathrm{ppm}$. Due to their relative integral of to one-to-two, the signals at 2.19 and $2.22 \mathrm{ppm}$ were assigned to another TMP species; possibly the sodium phenolate. The assignment of the remaining 


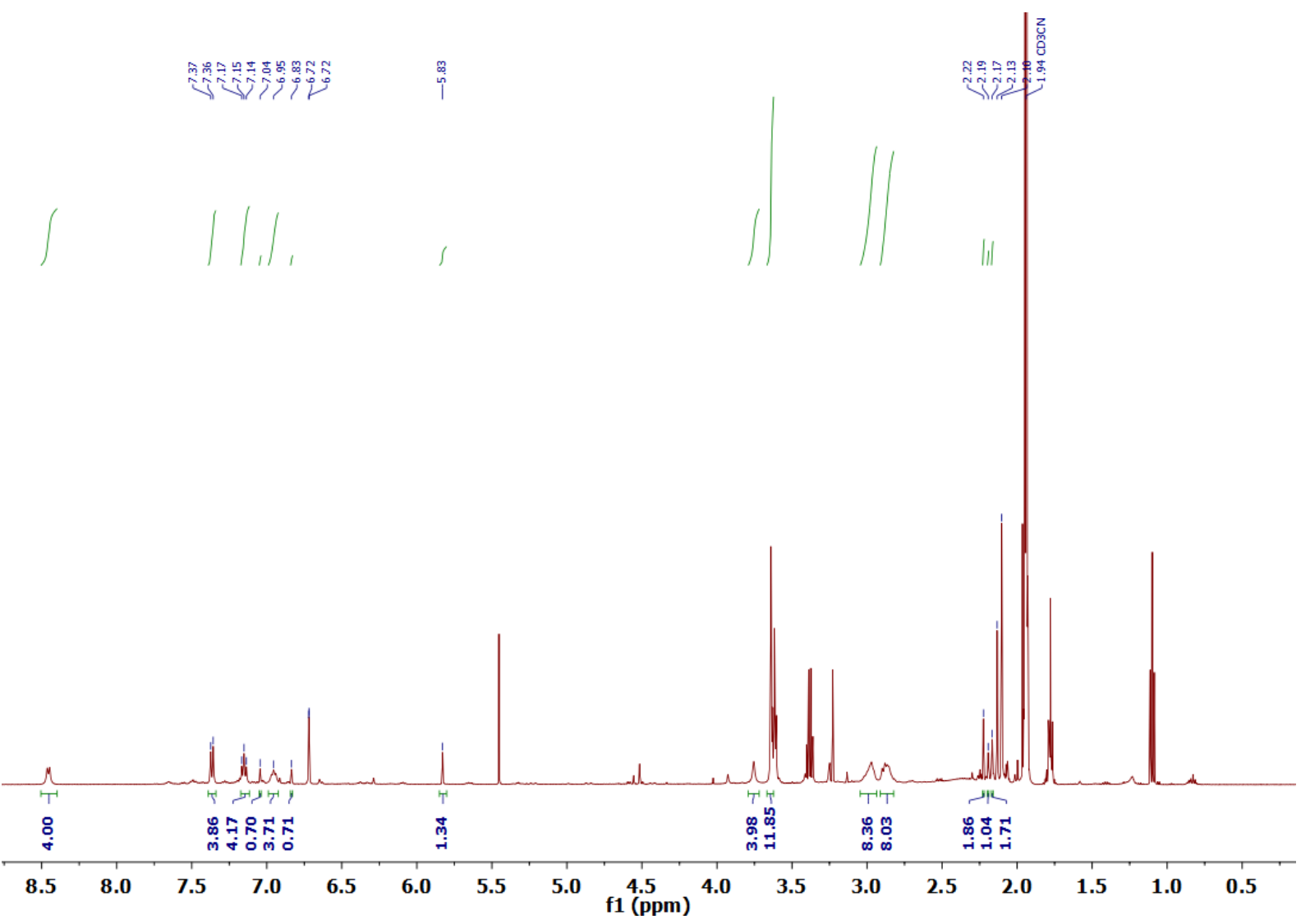

Figure 5.26 ${ }^{1} \mathrm{H}-\mathrm{NMR}$ spectrum of 4 and two equivalents of NaTMP in $\mathrm{CD}_{3} \mathrm{CN}$ under inert conditions.

signals could not be done unambigiously. Further ${ }^{13} \mathrm{C}-\mathrm{NMR}$ measurements as well as two-dimensional spectra of the mixture could prove useful in determining the residing species.

\section{Reactivity of quinone methides}

The reactivity of para-quinone methides is that of Michael acceptors undergoing 1,6-conjugate addition. This addition requires a nucleophile to attack the methide carbon. In the present case, the added atom stems from aerial dioxygen that does not act as a nucleophile. REEDIJK and coworkers observed formation of HDB from trimethyl phenol in a two step mechanism. $\frac{154}{154}$ First one nucleophile (in most cases an alcohol) added to the para- $\mathrm{CH}_{2}$ group. Then another nucleophile attacked the same position leading to an acetale function in para-position, that was immediately hydrolyzed to yield the benzaldehyde and liberate the two nucleophiles. Other groups witnessed the same behaviour. 1512281229$]$ HEIJ proposed a radical mechanism for the reaction of dioxygen with a para-methyl radical with the interaction of a metal ion binding the dioxygen and a resulting benzaldehyde and a metal hydroxide. ${ }^{230}$ This scenario does not seem to apply in the present case, as no radical was observed at any point during the reaction. 
JI and coworkers investigated the oxidation of para-substituted phenols in presence of $\mathrm{Cu}(\mathrm{OAc})_{2}$ and found ${ }^{18} \mathrm{O}$-incoporation. ${ }^{226}$. The incorporation was explained by reduction of molecular oxygen to water by the copper salt and incorporation of the newly formed ${ }^{18} \mathrm{OH}_{2}$ into the product.

This explanation might be valid also in the case at hand. From experiment it is known, that dioxygen is reduced to water by the dicopper complex. The reduction at the metal center in proximity to the oxidized substrate might lead to a direct reaction with the newly formed water/hydroxide. An experiment with $\mathrm{H}_{2}^{18} \mathrm{O}$ under anaerobic conditions might help to validate or falsify this assumption.

\section{Concluding remarks}

In this chapter the synthesis and characterization of dicopper(II) and dicopper(I) complexes of $\mathbf{H L}^{2}$ was described. The dicopper(I) complex $\mathbf{6}$ was tested for direct activation of dioxygen and a shortlived intermediate was observable by UV/Vis spectroscopy.

The dicopper(II) complex $\mathbf{4}$ was investigated towards it ability to catalitically oxidize TMP and was found to catalyze the para $\mathrm{C}-\mathrm{C}$ coupling to give TMBB under anaerobic and TMSQ under aerobic conditions when one equivalent of NaTMP was added (as a base). When two equivalents of NaTMP were added to 4 in MeCN, HDB was formed catalytically with a yield of up to $95 \%$. Kinetic aspects and the mechanism behind the transformation of TMP to HDB by 4 were investigated by means of $\mathrm{UV} / \mathrm{V}$ is, resonance Raman, EPR and NMR spectroscopy and ESI mass spectrometry as well as an oxygen uptake experiment. A quinone methide species was discussed as the possible intermediate in the process of forming HDB. 


\section{Structure and reactivity of dicopper complexes of $\mathrm{HL}^{3}$}

In the design of this ligand, the orientation of the imidazoles was changed from a connection of the 2-position to the backbone, to a connection of the 4-position, with the 2-position now bearing a phenyl group that is pointing outwards. These phenyl rings were anticipated to shield the active site and create a well defined environment around the active center to direct reactivity and prevent side reactions.

\subsection{Synthesis and characterization of dicopper(II) complexes of $\mathrm{HL}^{3}$}

The synthesis of the dicopper(II) complex was achieved by deprotonation of $\mathbf{H L}^{3}$ with $\mathrm{KO}^{t} \mathrm{Bu}$ in $\mathrm{MeOH}$ and subsequent addition of two equivalents of $\mathrm{Cu}\left(\mathrm{NO}_{3}\right)_{2} \cdot 3 \mathrm{H}_{2} \mathrm{O}$ (Scheme 6.1). Purification was done by dissolution of the complex in MeCN and slow
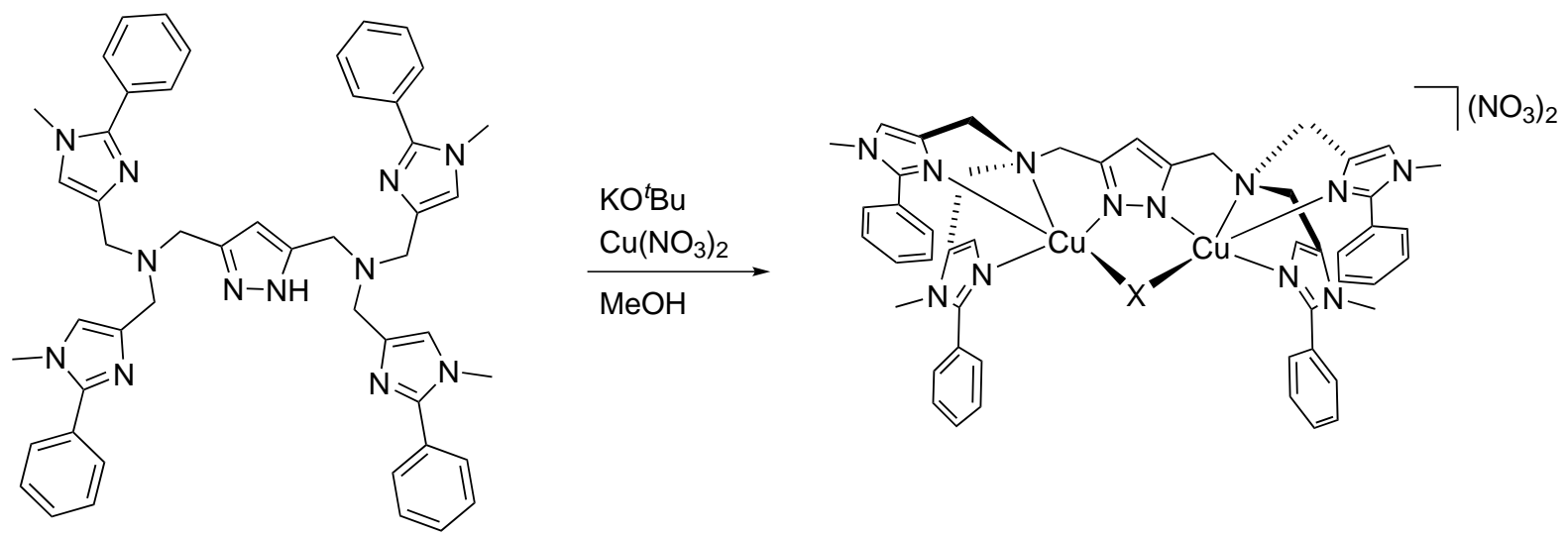

Scheme 6.1 Schematic representation of the complex synthesis

diffusion of $\mathrm{Et}_{2} \mathrm{O}$. After standing for 2-3 days, a green precipitate formed and was isolated for further studies. Crystallisation attempts were mostly unsuccessful with a variety of combinations of solvents, counterions and additives for cocrystallization, such as acetate, benzoic acid or other aromatic compounds. Nevertheless, a structure was obtained and will be discussed later in this section.

An ESI mass spectrum in MeCN shows various dicopper(II) species with different counterions (Figure 6.1). The main peak at $m / z=488.1$ could be assigned to $\left[\mathrm{L}^{3} \mathrm{Cu}_{2}(\mathrm{HCOO})\right]^{2+}$. Formic acid was added by the service department as a source of protons. Other The peak at $m / z=503.1$ was assigned to $\left[\mathrm{L}^{3} \mathrm{Cu}_{2}\left((\mathrm{OH}) \mathrm{H}_{2} \mathrm{CCOO}_{2}\right)\right]^{2+}$. 


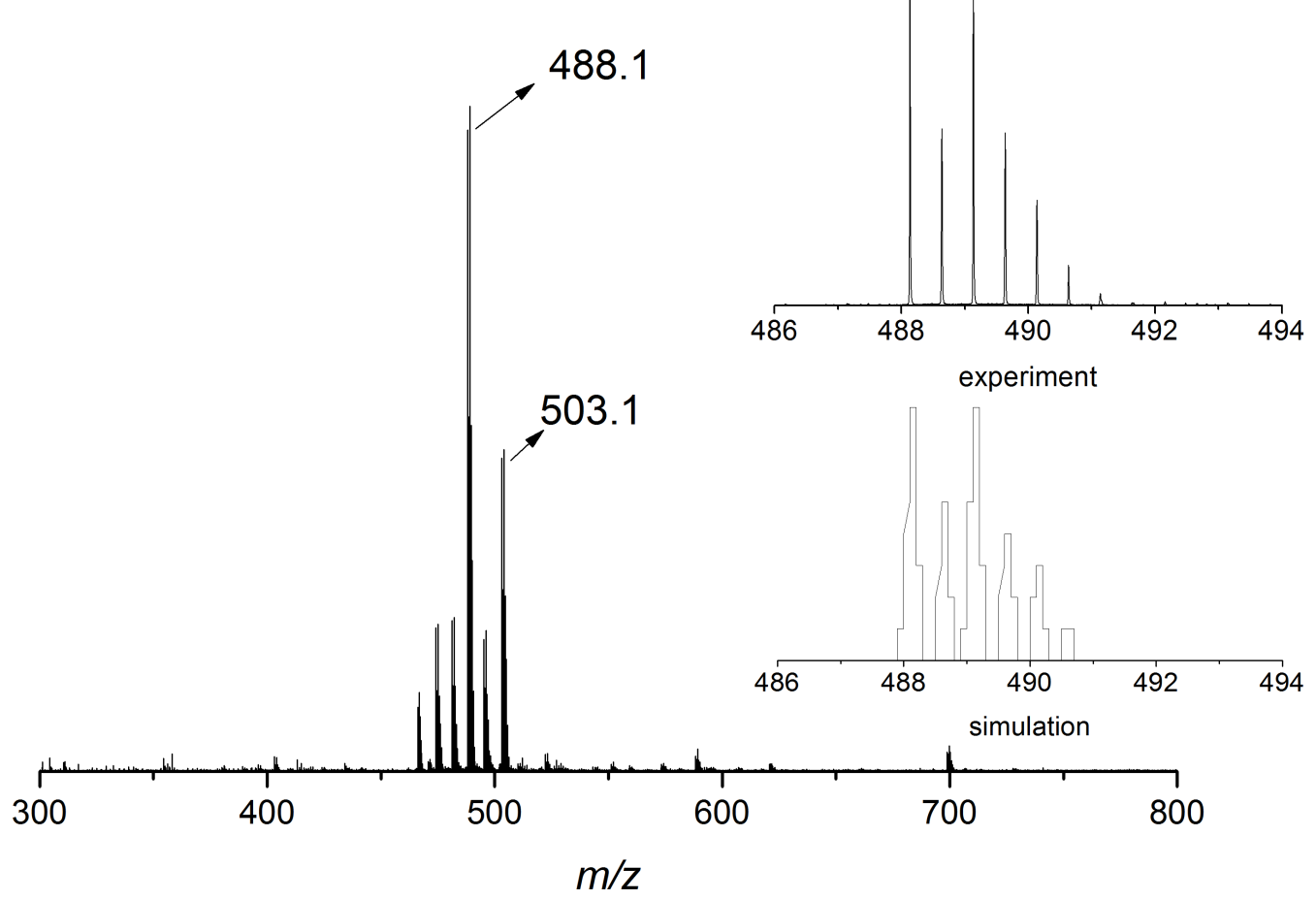

Figure 6.1 ESI mass spectrum of a solution of $\mathbf{7}$ in MeCN. Inset shows enlargement of the main peak at $m / z=488.1$ and simulation of $\left[\mathbf{L}^{3} \mathrm{Cu}_{2}(\mathrm{HCOO})\right]^{2+}$. The formate ion stems from formic acid added by the service department.

A UV/Vis absorption spectrum of $\mathbf{L}^{3} \mathrm{Cu}_{2}^{\mathrm{II}}\left(\mathrm{NO}_{3}\right)_{3}(\mathbf{7})$ is depicted in Figure 6.2. $\mathrm{A}$

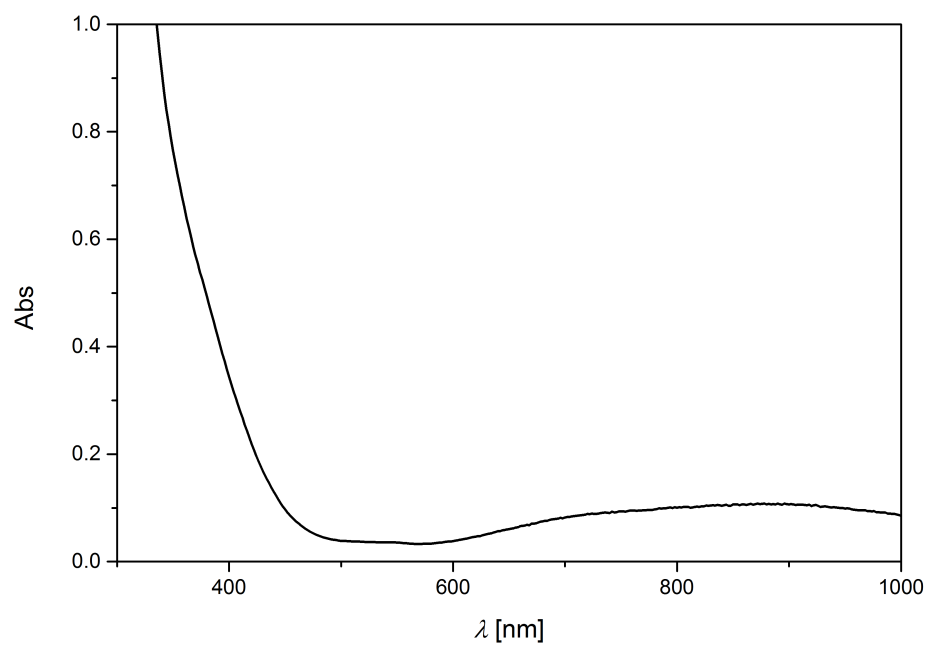

Figure 6.2 UV/Vis spectrum of $\mathbf{L}^{3} \mathrm{Cu}_{2}^{\mathrm{II}}\left(\mathrm{NO}_{3}\right)_{3}(\mathbf{7})\left(\mathrm{MeCN}, c=10^{-3} \mathrm{M}\right.$ at $20^{\circ} \mathrm{C}$.)

strong absorption feature below $430 \mathrm{~nm}$ is observed. This band was assigned to ligand based $\pi-\pi^{*}$ transitions. A broad band is found at $900 \mathrm{~nm}$ with a shoulder at $730 \mathrm{~nm}$. 
Both were assigned to $d-d$ transitions due to their low intensity and broadness.

A single crystal suitable for x-ray diffraction was obtained after leaving the crystallization mixture standing for severals weeks at room temperature (Figure 6.3). $\mathrm{Cu}\left(\mathrm{ClO}_{4}\right)_{2} \cdot 6 \mathrm{H}_{2} \mathrm{O}$ was used as the copper source, therefore perchlorates were found to
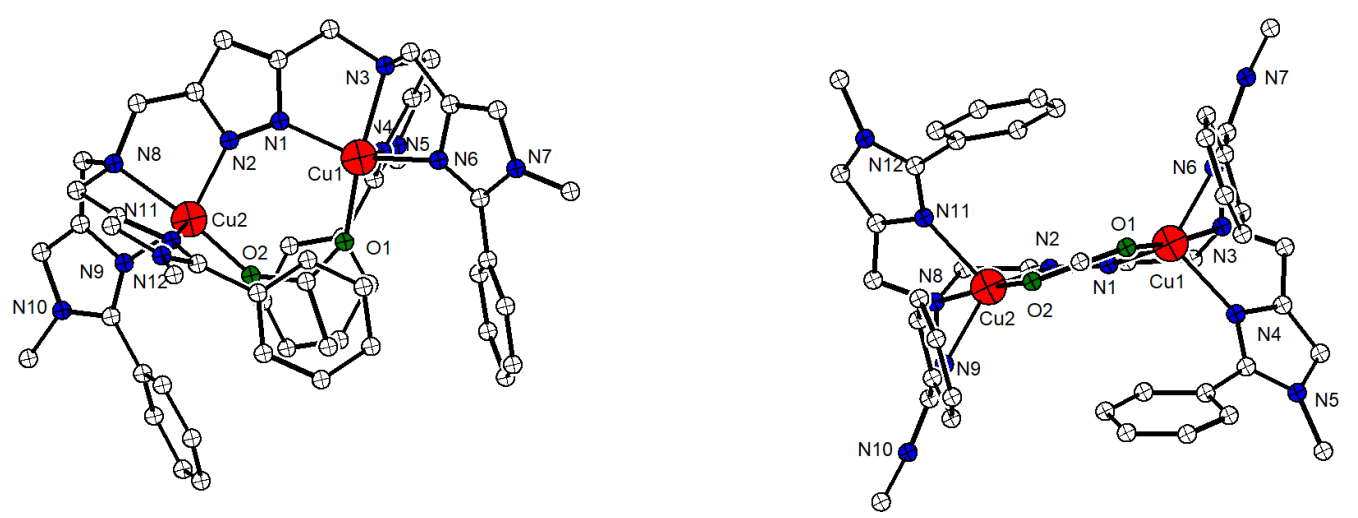

Figure 6.3 Molecular structure of the cation of 8 together with a partial labeling scheme; thermal displacement ellipsoids are drawn at $50 \%$ probability. Hydrogen atoms and counterions have been omitted for clarity. Left) View along the z-achses. Right) View along the $\mathrm{x} / \mathrm{y}$ achses.

be the counterions of the complex. The origin of the acetate found in the structure is unknown as it was not added intentionally. Any attempts to recreate the crystallization with deliberate addition of acetate failed.

The molecular structure revealed a box like arrangement of the phenyl rings of the imidazole sidearms. The desired shielding of the binding site was thus achieved. The copper ions are found $4.16 \AA$ apart. Each copper ion is five-fold coordinated in a highly distorted trigonal bipyramidal fashion $(\tau=0.66)$. They each diverge by $15.3^{\circ}$ from the plane of the pyrazolate. The copper-copper distance is $4.16 \AA$.

\subsection{Synthesis, characterization and reactivity of a dicopper(I) complex of $\mathrm{HL}^{3}$}

The synthesis of the dicopper(I) complex proceeds via deprotonation of the ligand $\mathbf{H L}^{3}$ with a strong alkoxide base like $\mathrm{KO}^{t} \mathrm{Bu}$ in $\mathrm{MeCN}$ with subsequent addition of two equivalents of $\left.\left[\mathrm{Cu}(\mathrm{MeCN})_{4}\right)\right]$ OTf to give the desired compound $\mathbf{L}^{3} \mathrm{Cu}_{2}^{\mathrm{I}} \mathrm{OTf}(\mathbf{9})$.

ESI mass spectrometry revealed the existence of the target complex $\left[\mathrm{L}^{3} \mathrm{Cu}_{2}\right]^{+}$Figure 6.4). The main peak at $m / z=466.3$ could be assigned to $[\mathrm{M}+\mathrm{H}]^{2+}$, while the $[\mathrm{M}]^{+}$ species is found at $m / z=931.3$.

Recorded NMR spectra were of little help to investigate the complex because spectra 


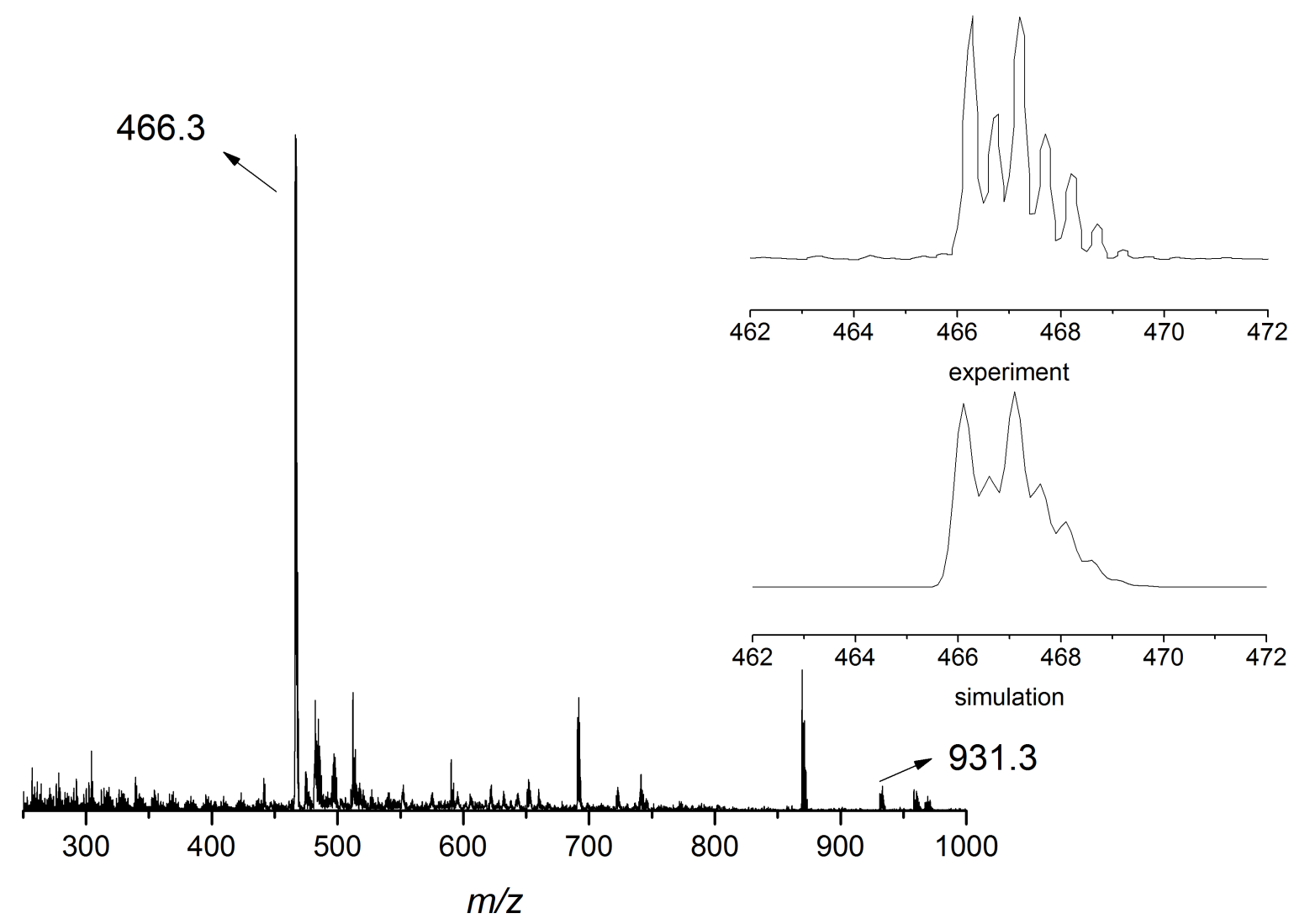

Figure 6.4 ESI mass spectrum of a solution of $\left[\mathbf{L}^{3} \mathrm{Cu}_{2}^{\mathrm{I}}\right] \mathrm{OTf}(\mathbf{9})$ in MeCN measured from a glovebox. Inset shows enlargement of the peak at $\mathrm{m} / z=466.3$ and simulation of $\left[\mathbf{L}^{3} \mathrm{Cu}_{2}+\mathrm{H}\right]^{2+}$.

of the diamagnetic dicopper(I) complex 9, as well as for the additionally synthesized $\left[\mathrm{L}^{3} \mathrm{Cu}_{2}^{\mathrm{I}}\right] \mathrm{BF}_{4}$, recorded in $\mathrm{MeCN}$ and acetone at temperatures between $-30{ }^{\circ} \mathrm{C}$ and $+70{ }^{\circ} \mathrm{C}$, only gave broad and overlapping signals that revealed no relevant information about the complex. A possible explanation of this behaviour is pronounced flexibility and dynamic behaviour of the complex in solution.

\subsection{Reactivity with $\mathrm{O}_{2}$}

To investigate the possibility of oxygen activation by the dinuclear copper(I) complex 9, UV/Vis experiments were carried out. 9 was dissolved in dry EtCN in a glovebox, transferred into a UV/Vis cuvette with a screw cap and septum and transferred out of the glovebox into a spectrometer, where it was cooled to $-70{ }^{\circ} \mathrm{C}$. When the desired temperature was reached, dry dioxygen was bubbled through the solution with a syringe. At this temperature, no change in the absorption spectrum was observed (Figure 6.5). To investigate a possible reaction at higher temperatures, the temperature was elevated gradually. No change was observed until $-20{ }^{\circ} \mathrm{C}$ were reached. At this point the dicopper(I) complex was oxidized to a dicopper(II) complex (Figure 6.5). This behaviour 


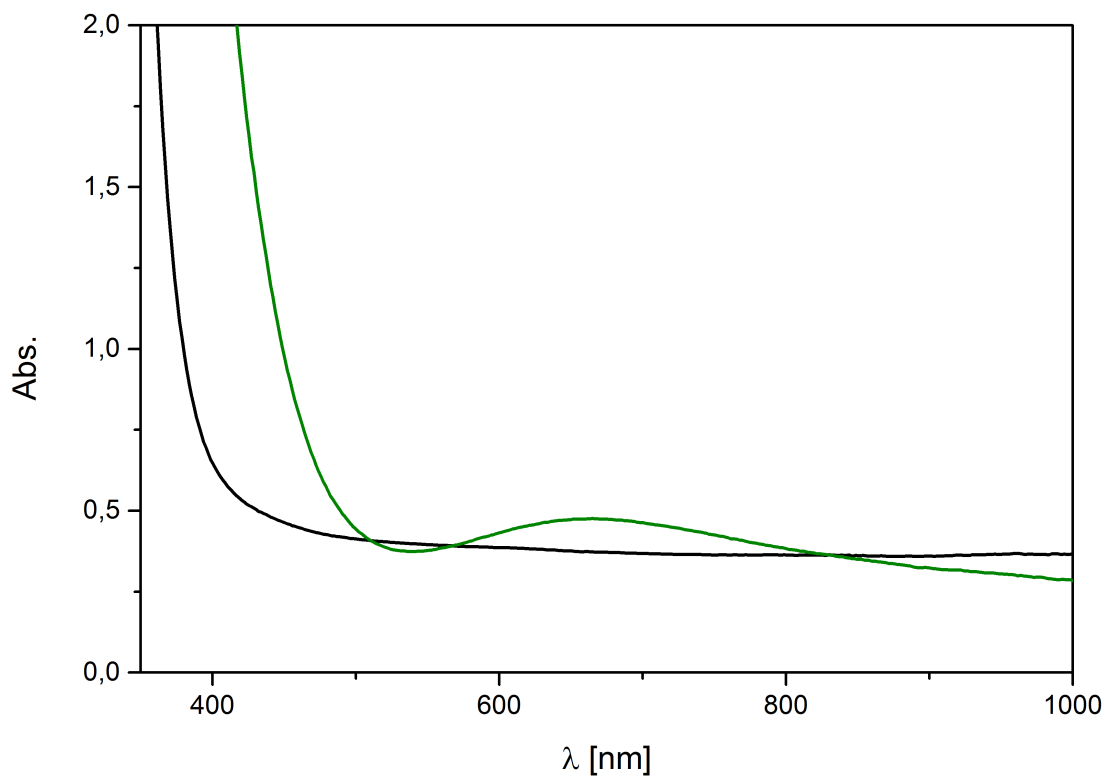

Figure 6.5 black) UV/Vis spectrum of $\mathbf{L}^{3} \mathrm{Cu}_{2}^{\mathrm{I}} \mathrm{OTf}\left(c=5 \cdot 10^{-3} \mathrm{M}\right)$ in the presence of $\mathrm{O}_{2}$ in acetone at $-70^{\circ} \mathrm{C}$. green) $\mathrm{UV} / \mathrm{Vis}$ spectrum of the oxidized complex at $-20^{\circ} \mathrm{C}$ in acetone.

was also observed for $\left[\mathbf{L}^{3} \mathrm{Cu}_{2}\right] \mathrm{BF}_{4}$ and for both complexes also in acetone and MeCN solution. In conclusion, it was not possible to trap an intermediate that would be indicative of direct activation of dioxygen by a dinuclear copper(I) complex of $\mathbf{H L}^{3}$.

An alternative route for generating ta putative dicopper(II)-peroxo complex, that is usually obtained by reaction of a dicopper(I) complex with dioxygen, is a reverse approach, wherein the dicopper(II) complex is treated with a peroxide. However, when the dicopper(II) complex was treated with hydrogen peroxide at $-30{ }^{\circ} \mathrm{C}$, no change was evident in the UV/Vis spectrum. The absence of reactivity also persisted when the temperature was elevated to ambient level. The same behaviour was witnessed for other peroxides, such as tert-butylhydroperoxide.

\subsection{Reactivity with Sulfur}

The dicopper(I) and dicopper(II) complex of $\mathbf{H L}^{3}$ have been tested for reaction with elemental sulfur. Unfortunately, however, neither the copper(II) nor the copper(I) complex showed any change in their $\mathrm{UV} / \mathrm{V}$ is absorption spectrum, when $\mathrm{S}_{8}$ dissolved in $\mathrm{Et}_{2} \mathrm{O}$ was added.

In the same manner as before, the reverse approach of adding sulfide to the dicopper(II) complex was used to potentially generate the desired sulfide complex. Lithium sulfide was chosen as a substrate due to its good solubility in MeCN. UV/Vis experiments 
were carried out in $\mathrm{MeCN}$ starting from $-35^{\circ} \mathrm{C}$. Upon addition of one equivalent of lithium sulfide, a band at $400 \mathrm{~nm}$ rises (Figure 6.6). The $d$ - $d$ band at $690 \mathrm{~nm}$ does not shift. When the temperature is increased stepwise to $15^{\circ} \mathrm{C}$ no significant change in the spectrum is observed. When two equivalents of sulfide are present, at $-70^{\circ} \mathrm{C}$ only subtle

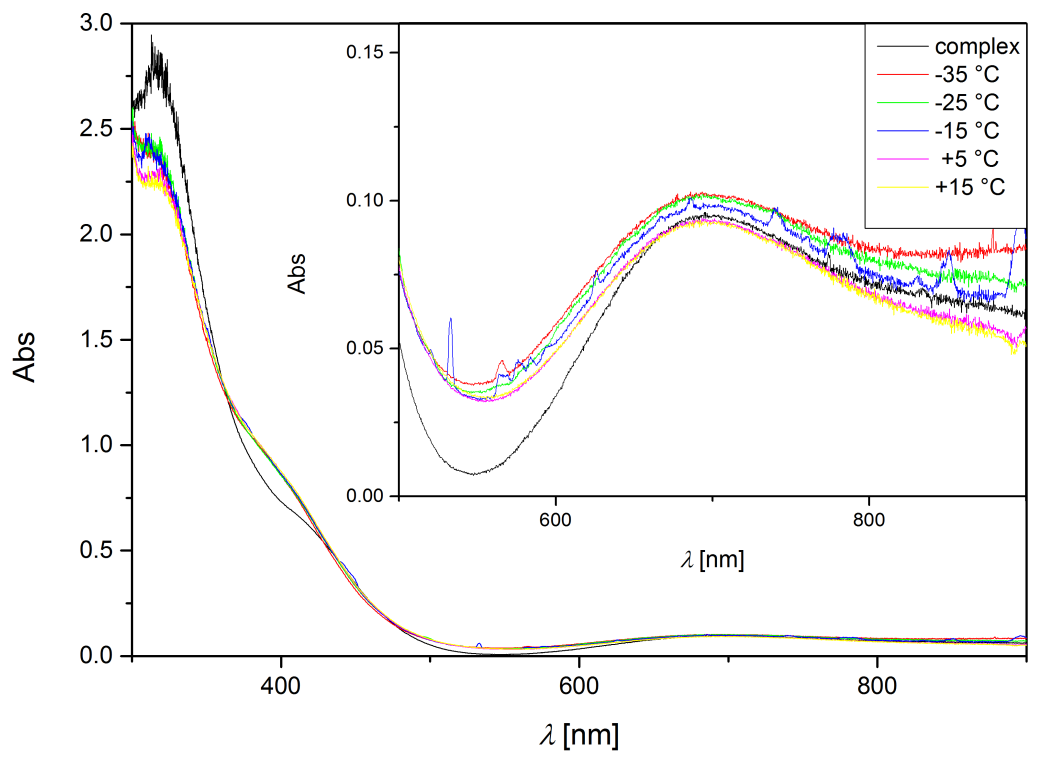

Figure 6.6 UV/Vis spectra of $7\left(c=5 \cdot 10^{-4} \mathrm{M}\right)$ and $\mathrm{Li}_{2} \mathrm{~S}\left(c=5 \cdot 10^{-4} \mathrm{M}\right)$ in $\mathrm{MeCN}$ at increasing temperatures starting from $-35^{\circ} \mathrm{C}$.

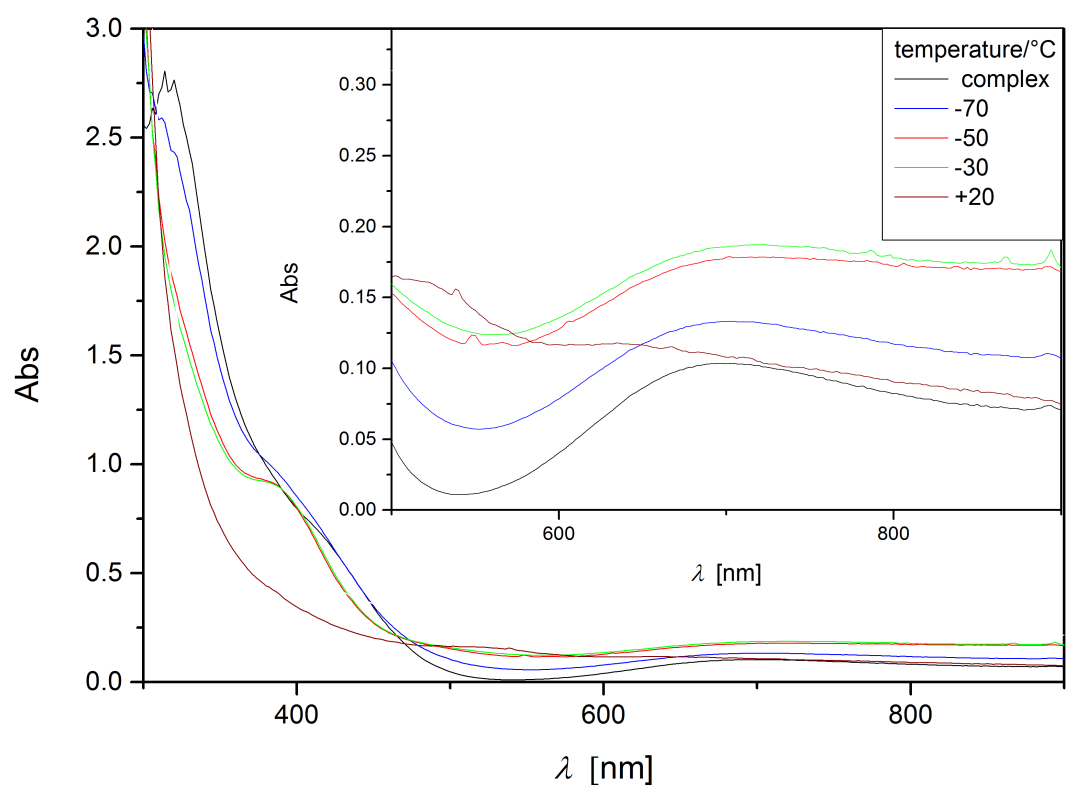

Figure 6.7 UV/vis spectra of $7\left(c=5 \cdot 10^{-4} \mathrm{M}\right)$ and $\mathrm{Li}_{2} \mathrm{~S}\left(c=1 \cdot 10^{-3} \mathrm{M}\right)$ in propionitrile at increasing temperatures starting from $-70^{\circ} \mathrm{C}$.

changes are visible (Figure 6.7). A band at $400 \mathrm{~nm}$ rises slightly and the band at 316 $\mathrm{nm}$ decreases a bit. Upon further waming of the solution to $-50{ }^{\circ} \mathrm{C}$ the band at $400 \mathrm{~nm}$ is more pronounced due to a larger decrease of the band at $316 \mathrm{~nm}$. The $d-d$ at $690 \mathrm{~nm}$ 
band does not shift during the process. Also further warming to $-30{ }^{\circ} \mathrm{C}$ does not alter the spectrum. Only a baseline shift is observed. When the solution reaches a threshold of about $10{ }^{\circ} \mathrm{C}$ the colour of the solution vanishes along with the spectroscopic features of the dicopper(II) species. This indicates the reduction of the complex to its dicopper(I) state.

Figure 6.8 shows the change in absorption in $\mathrm{MeCN}$ at $10^{\circ} \mathrm{C}$ with increasing addition

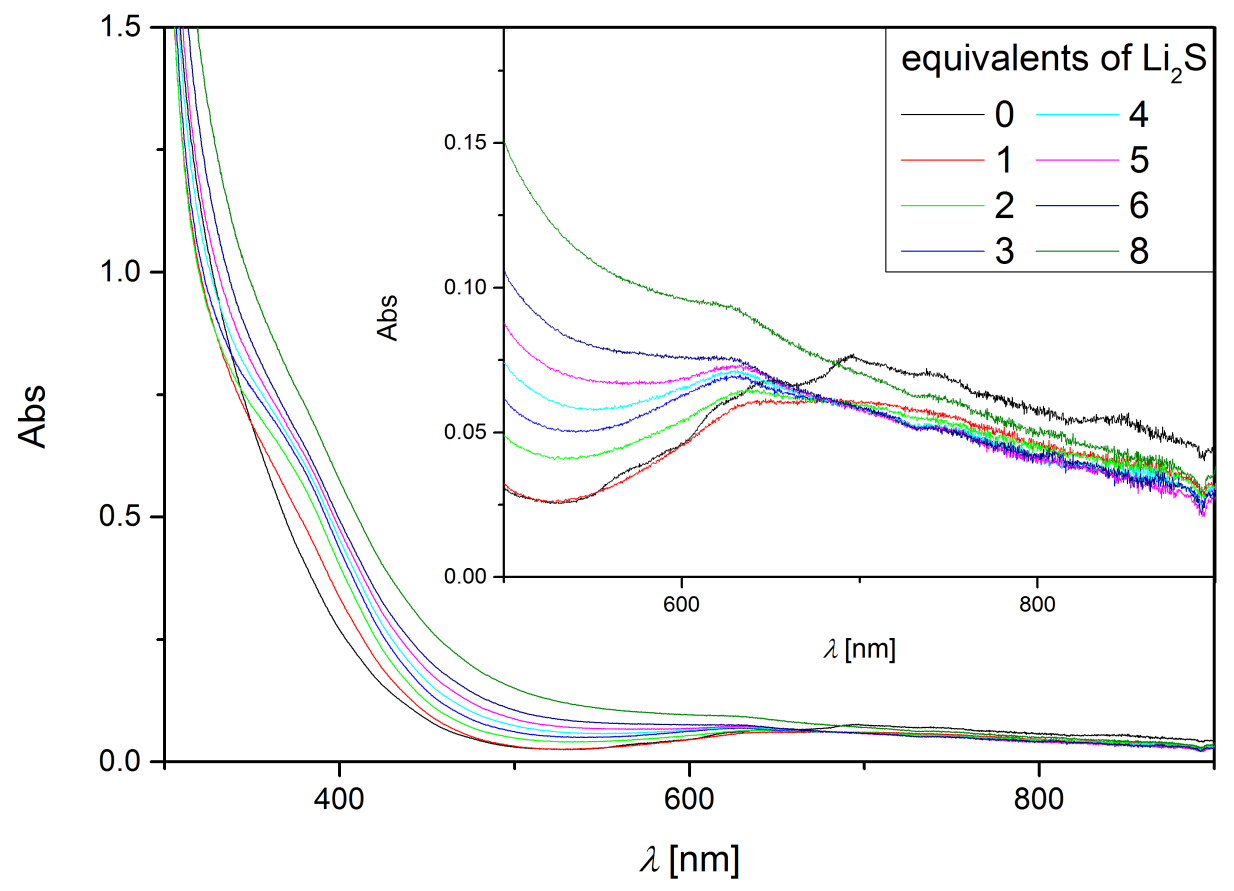

Figure 6.8 UV/Vis spectra of $7\left(\mathrm{MeCN}, c=5 \cdot 10^{-4} \mathrm{M}\right)$ and increasing equivalents of $\mathrm{Li}_{2} \mathrm{~S}$.

of equivalents. The already known band at $400 \mathrm{~nm}$ is again present and increases with further addition of lithium sulfide; the $d$ - $d$ band at $620 \mathrm{~nm}$ remains unaffected.

In conclusion, there is not enough evidence for the formation of a new species, especially because there is no shift of the $d-d$ band and therefore no change in the coordination environment of the metal. 


\subsection{Reactivity with NO}

In the literature, very few examples of copper-NO species exist, although their synthesis follows simple procedures. ${ }^{231}$ 237 In this light, different NO-donors have been tried to react with the dicopper(I) and the copper(II) complexes.

$\mathrm{Ph}_{3} \mathrm{SCNO}$ was first tried as an easy to handle NO source. It will not release $\mathrm{NO}$ spontaneously but only in a redox reaction with copper(II). 238. However, no such reaction took place and both compounds remained unaltered in the solution as $\mathrm{UV} / \mathrm{Vis}$ spectroscopy indicated.

Secondly, $\mathrm{NOBF}_{4}$, a moisture sensitive white solid, strong oxidant and $\mathrm{NO}^{+}$donor did show a result upon reaction with the dicopper(I) species (Figure 6.9). There are

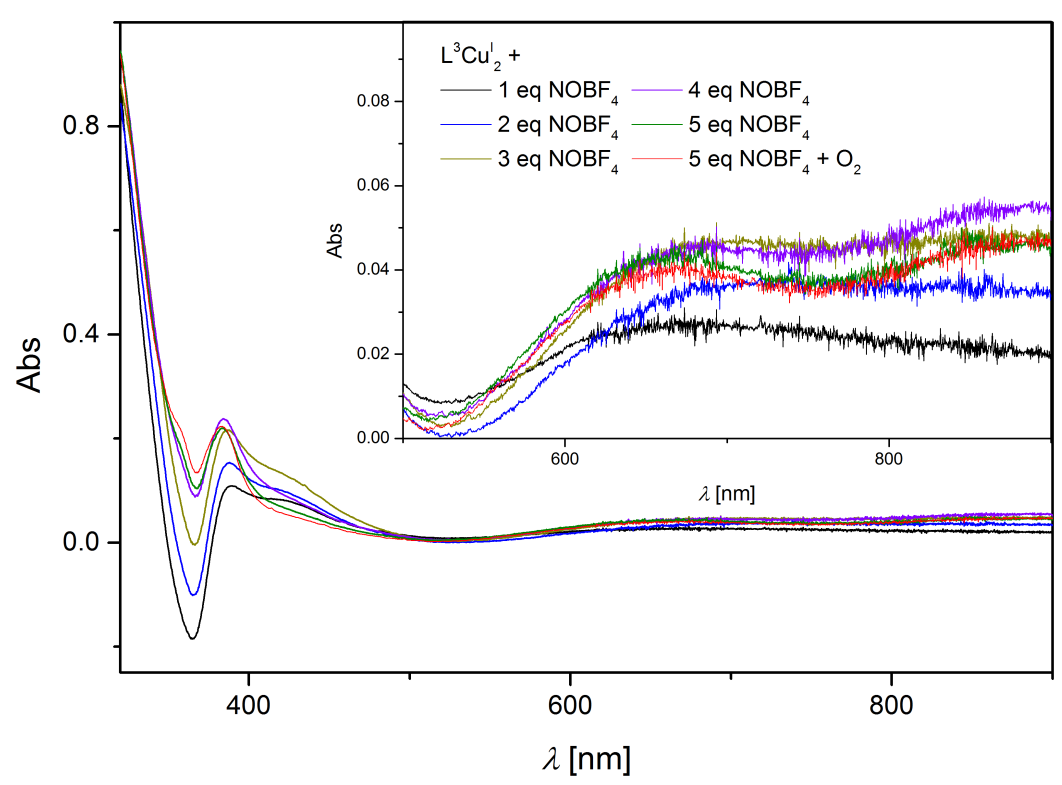

Figure 6.9 UV/Vis spectra of $\mathbf{L}^{3} \mathrm{Cu}_{2}^{\mathrm{I}} \mathrm{OTf}$ in $\mathrm{MeCN}\left(c=5 \cdot 10^{-4} \mathrm{M}\right)$ with increasing equivalents of $\mathrm{NOBF}_{4}$.

significant changes around 310 and $400 \mathrm{~nm}$ that follow no obvious pattern. A clearer indication of a reaction is the rise of a previously absent $d-d$ band at $640 \mathrm{~nm}$. The dicopper(II) complex shows a $d$ - $d$ absorption feature at $690 \mathrm{~nm}$, so this band is likely to originate from a newly generated species. Further addition of $\mathrm{NOBF}_{4}$ led to an increase in the absorption of the $d-d$ band until three equivalents were added. Adiition of more $\mathrm{NOBF}_{4}$ did not change the spectrum significantly. Furthermore, opening the sealed vessel to the atmosphere does not have any influence on the absorption spectrum. Solution IR spectra of $\left[\mathbf{L}^{3} \mathrm{Cu}_{2}^{\mathrm{I}}\right] \mathrm{OTf}$ in $\mathrm{MeCN}$ and one equivalent of $\mathrm{NOBF}_{4}$ are shown in Figure 6.10. There is no evidental change in the spectrum after addition of $\mathrm{NOBF}_{4}$ and no expected NO stretching frequency has been observed. 


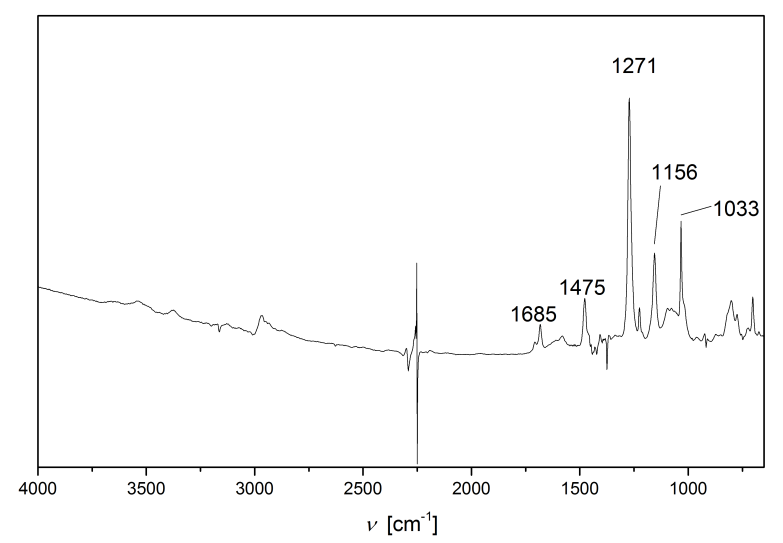

(a) Solution IR spectrum of $\left[\mathbf{L}^{3} \mathrm{Cu}_{2}^{\mathrm{I}}\right] \mathrm{Otf}$ in $\mathrm{MeCN}$.

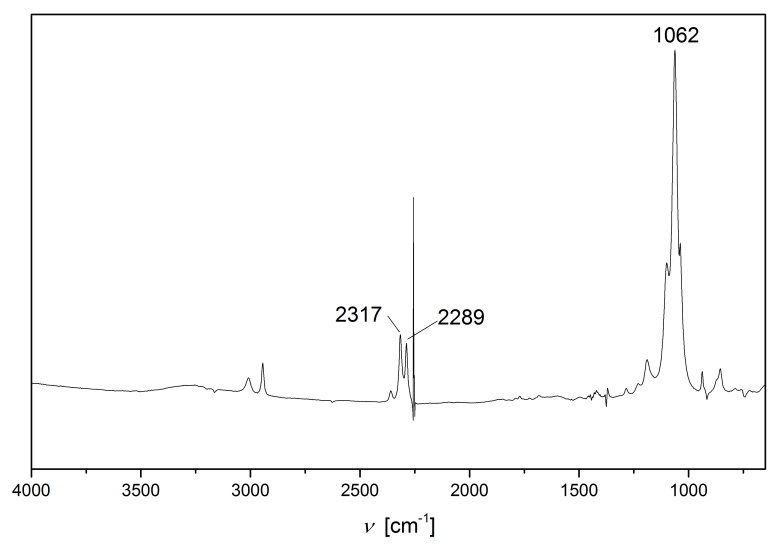

(c) Solution IR spectrum of $\mathrm{NOBF}_{4}$ in $\mathrm{MeCN}$.

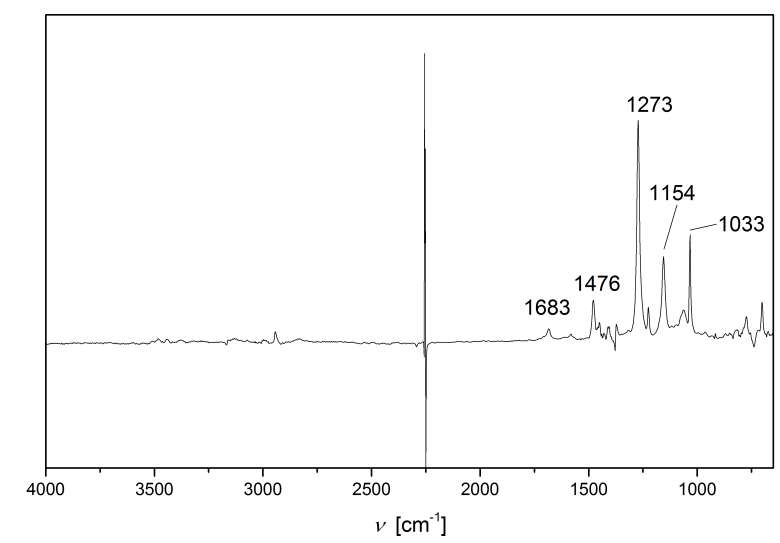

(b) Solution IR spectrum of $\mathbf{L}^{3} \mathrm{Cu}_{2}^{\mathrm{I}} \mathrm{Otf}$ and one equivalent of $\mathrm{NOBF}_{4}$ in $\mathrm{MeCN}$.

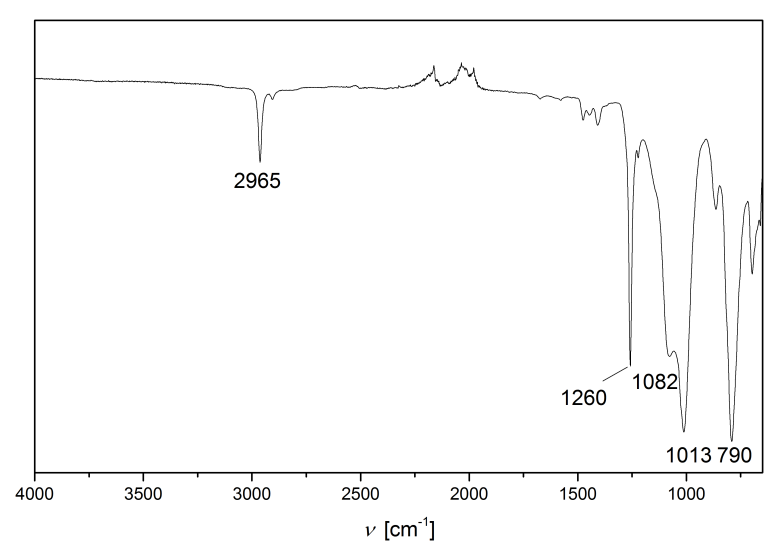

(d) Solid state IR spectrum of $\left[\mathbf{L}^{3} \mathrm{Cu}_{2}^{\mathrm{I}}\right] \mathrm{Otf}$ and one equivalent of $\mathrm{NOBF}_{4}$.

Figure 6.10 IR spectra of different solutions of $\mathbf{9}$ and $\mathrm{NOBF}_{4}$.

ESI-MS analysis showed only peaks with isotopic pattern characteristic for mononuclear copper species, indicating the decomposition of the complex due to the high oxidation potential of $\mathrm{NOBF}_{4}$.

In conclusion, the addition of $\mathrm{NOBF}_{4}$ to $\mathbf{9}$ does not lead to the formation of the desired NO species but to decompostion of the complex.

A further alternative is the direct reaction of the copper complex with gaseous NO. Experiments have been carried out with the copper(I) as well as the copper(II) complex. The respective solutions were prepared in a glovebox in a schlenk-flask. Gaseous NO was taken from a special apparatus with a dry ice/acetone cooling trap to remove other $\mathrm{NO}_{x}$ species. The colorless gas was taken from the apparatus via syringe through a septum and added to the solution of the dicopper complex through a septum. $\mathrm{UV} / \mathrm{Vis}$ spectra have been recorded at room temperature in MeCN. The copper(II) complex did not show any change in absorption, when gaseous NO was added. The dicopper(I) complex on the other hand underwent visible change, as can be seen in 


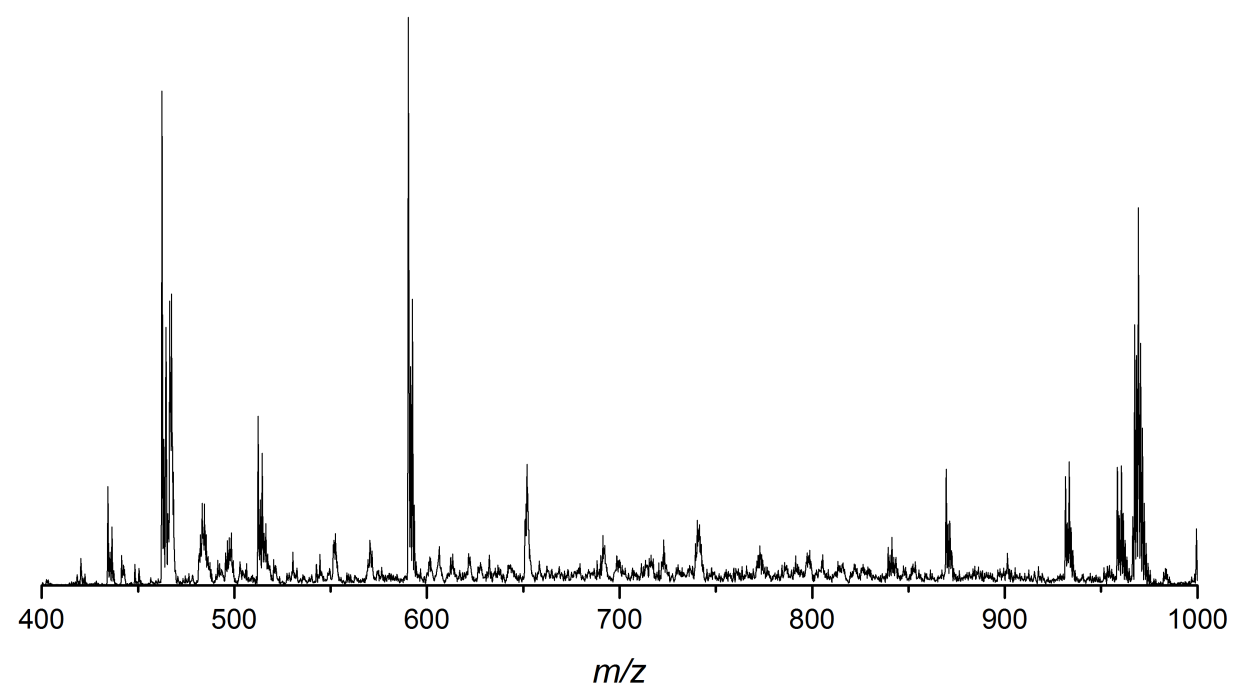

Figure 6.11 ESI mass spectrum of a solution of $\mathbf{9}$ and $\mathrm{NOBF}_{4}$ in MeCN. Mainly mononuclear copper species were observed.

\section{Figure 6.12.}

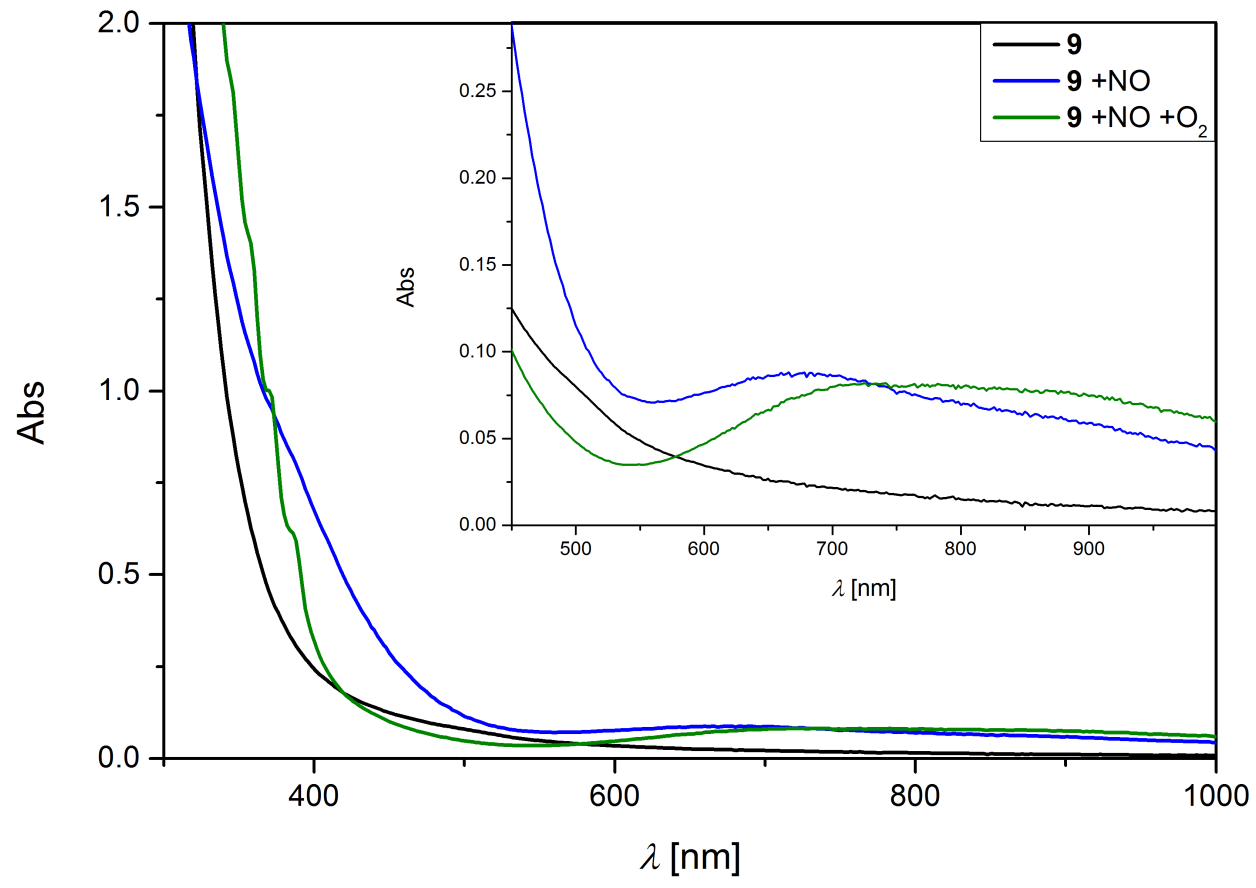

Figure 6.12 UV/Vis spectra of $\mathbf{9}\left(c=10^{-4} \mathrm{M}\right)$ with gaseous NO and oxygen in MeCN.

When the dicopper(I) complex is exposed to gaseous NO, a new band at $388 \mathrm{~nm}$ rises, along with a $d$-d band at $675 \mathrm{~nm}$ that shows a small shoulder at $875 \mathrm{~nm}$. When this species is exposed to dry dioxygen, the band at $388 \mathrm{~nm}$ decreases and the $d$ - $d$ band shifts 
to $735 \mathrm{~nm}$. The dicopper(II) complex shows $d-d$ FF features at $734 \mathrm{~nm}$ and $875 \mathrm{~nm}$. Since

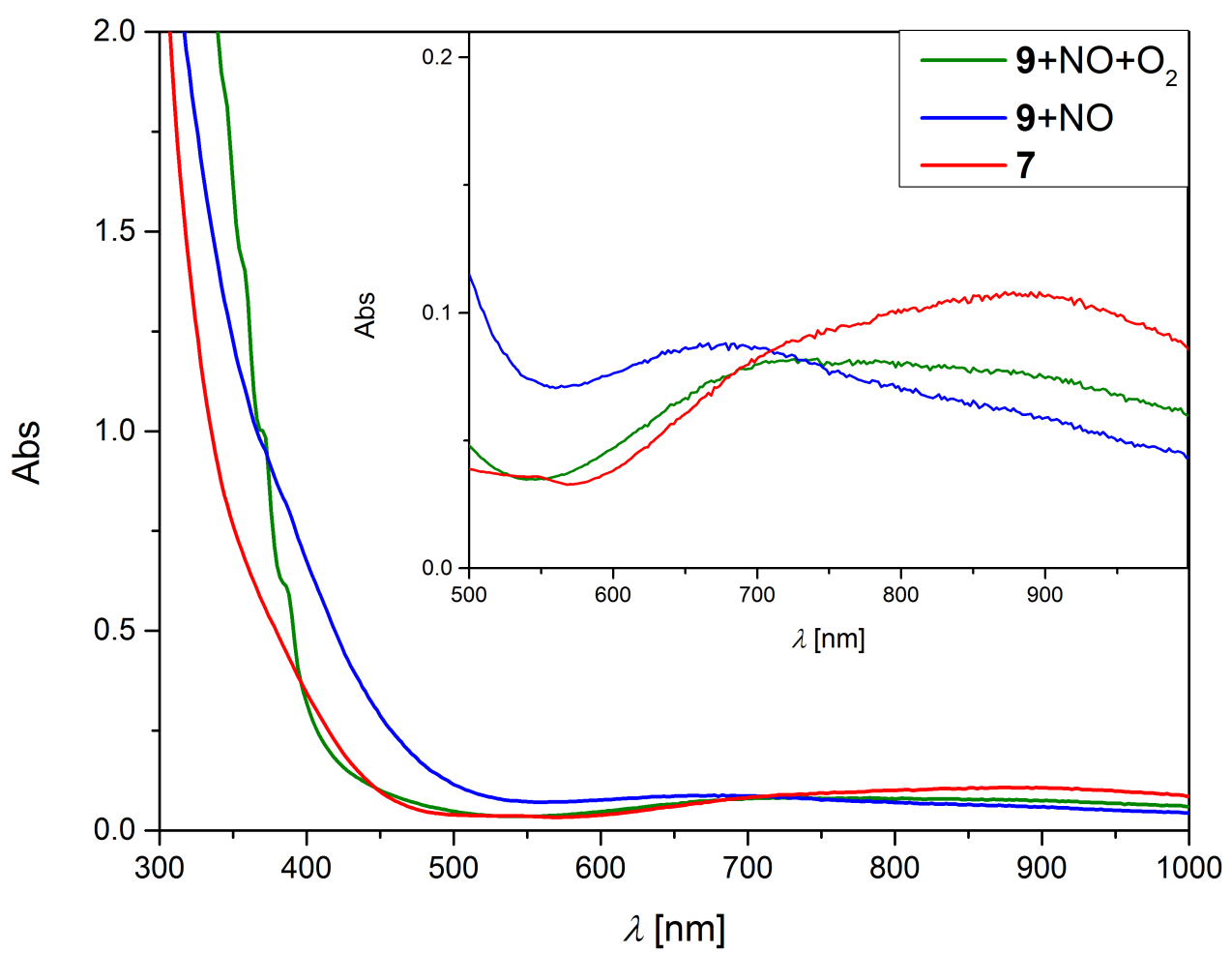

Figure 6.13 UV/Vis spectra of $9\left(c=10^{-4} \mathrm{M}\right)$ with gaseous NO and oxygen in MeCN

the spectroscopic properties for all species are different, the presence of two different NOcomplexes can be assumed in the presence and absence of dioxygen.

IR spectroscopy was chosen in the next step as it is a powerful tool for the identification of pronounced stretching bands like the NO stretch that can be found between $1500 \mathrm{~cm}^{-1}$ and $1800 \mathrm{~cm}^{-1}$.232|239|240 Solution IR spectroscopy of a solution containing $\mathbf{9}$ and gaseous NO 6.14c in MeCN revealed the presence of two new bands at 1088 and $1339 \mathrm{~cm}^{-1}$ compared to the spectrum of 9 6.10a. These are too low in energy for a $\mathrm{N}-\mathrm{O}$ stretching frequency. Also, a shift of the band at $1582 \mathrm{~cm}^{-1}$ present in the spectrum of $\mathbf{9}$ is shifted to $1618 \mathrm{~cm}^{-1}$ but could not be assigned.

KARLIN and coworkers very recently observed the formation of $\mathrm{N}_{2} \mathrm{O}_{2}^{2-}$ when exposing $\left[\mathrm{Cu}^{\mathrm{I}}(\text { tmpa })(\mathrm{MeCN})\right]^{+}$to gaseous NO. ${ }^{241}$ The stretching frequencies for transition metal stabilized $\mathrm{N}_{2} \mathrm{O}_{2}^{2-}$ are found around $1250-1390 \mathrm{~cm}^{-1}\left(1392 \mathrm{~cm}^{-1}\right.$ in aqueous solution) for the $\mathrm{N}=\mathrm{N}$ stretch and $1050-1150 \mathrm{~cm}^{-1}$ for the $\mathrm{N}-\mathrm{O}$ stretch. ${ }^{242} 245$ Therefore, the bands found at 1088 and $1339 \mathrm{~cm}^{-1}$ after reaction of $\mathbf{9}$ with gaseous NO fall well within the window of a $\mathrm{N}-\mathrm{O}$ and $\mathrm{N}=\mathrm{N}$ stretch of $\mathrm{N}_{2} \mathrm{O}_{2}^{2-}$.

When the solution was evaporated to dryness and a solid state IR was recorded (6.14d), these two band are no longer present and the band at $1618 \mathrm{~cm}^{-1}$ is shifted back to $1580 \mathrm{~cm}^{-1}$. This indicates the reversibility of a possible $\mathrm{N}_{2} \mathrm{O}_{2}$ binding process. To further investigate the assumed $\mathrm{N}_{2} \mathrm{O}_{2}$-species, an ESI mass spectrum was measured of the solu- 


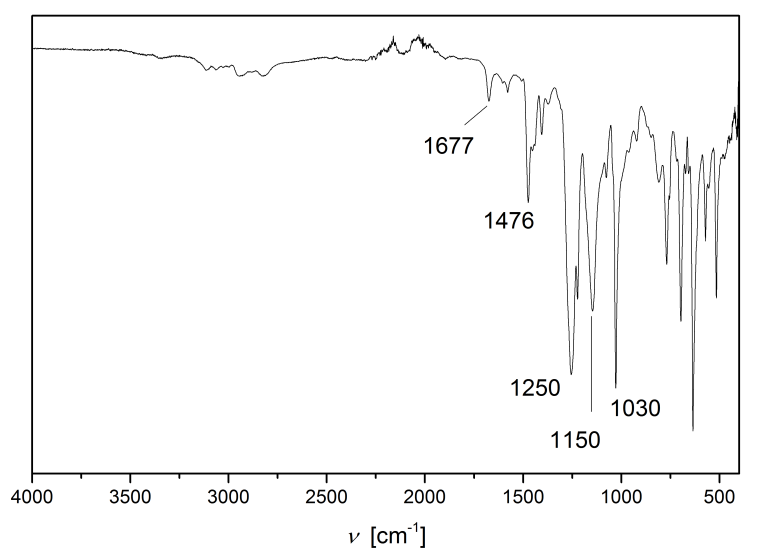

(a) Solid state IR spectrum of $\mathbf{9}$

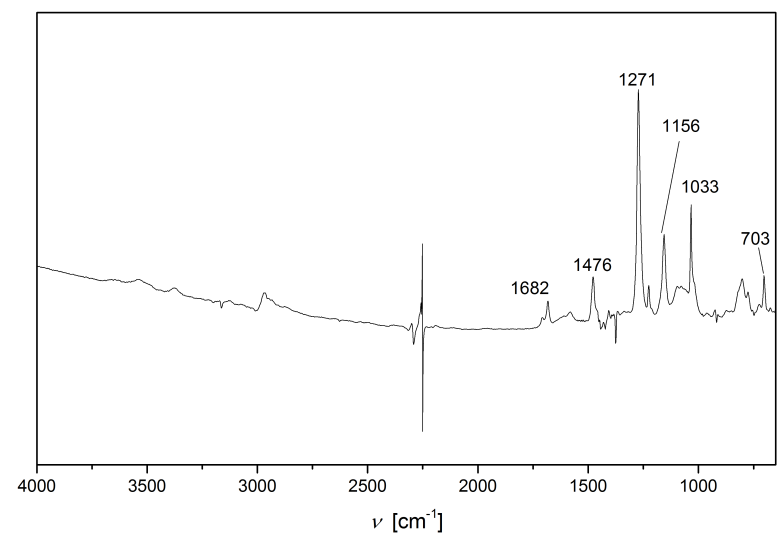

(c) Solution IR specrtrum in $\mathrm{MeCN}$ of $\mathbf{9}$ and gaseous NO.

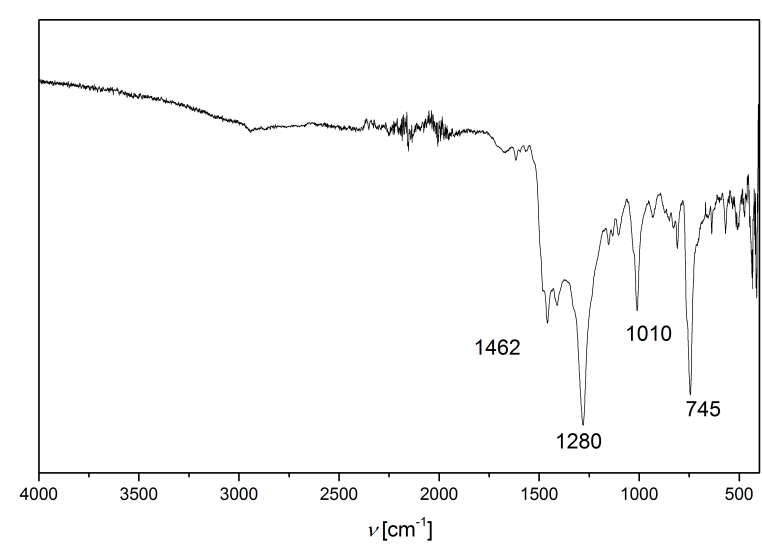

(b) Solid state IR spectrum of 7 .

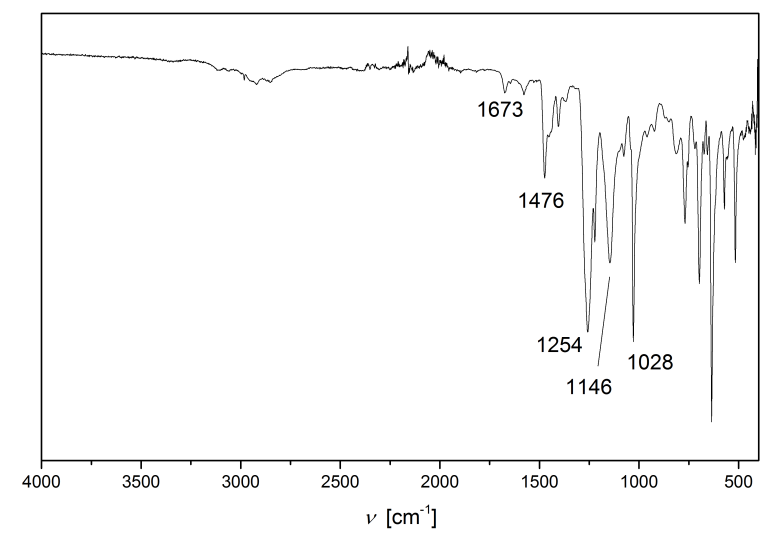

(d) Solid state IR spectrum of $\mathbf{9}$ and gaseous NO after evaporation of the solvent.

Figure 6.14 IR spectra of different solutions of $\mathbf{9}$ and gaseous NO

tion of 9 and gaseous NO in MeCN. The resulting spectrum is depicted in Figure 6.15. The main peak at $m / z=488.3$ was assigned to $\left[\mathbf{L}^{3} \mathrm{Cu}_{2}\left(\mathrm{NO}_{2}\right)\right]^{2+}$. It cannot be excluded that the $\mathrm{NO}_{2}$ species stems from reaction of $\mathrm{NO}$, or the initial intermediate resulting from 9 and NO with dioxygen. Because of its +2 charge, the copper ions could be assigned an oxidation state of + II with a negatively charged $\mathrm{NO}_{2}$-ion and one negative charge from the pyrazolate. Another species was found at $m / z=495.2$. Assignment of a hyponitrite bound to the complex $\left(\left[\mathbf{L}^{3} \mathrm{Cu}_{2}\left(\mathrm{~N}_{2} \mathrm{O}_{2}\right)\right]^{+}\right)$would lead to a $m / z$ of 495.64 that is half a $m / z$ unit too high. Also, this species would have a charge of $+1 ; 2-$ from the $\mathrm{N}_{2} \mathrm{O}_{2}$ and 1 - from the pyrazolate and +4 from two copper(II) ions. The addition of a proton to rationalize the charge of +2 would lead to a $m / z$ value of $496.14\left(\left(\left[\mathrm{~L}^{3} \mathrm{Cu}_{2}\left(\mathrm{~N}_{2} \mathrm{O}_{2}\right)+\mathrm{H}\right]^{2+}\right)\right.$ that is one $m / z$ value too large for a doubly charged species and two mass units for a singly charged species. Therefore, the observed species in the mass spectrum is unlikely to be a hyponitrite bound to the complex.

Another possible explanation is the exchange of the pyrazole $4-\mathrm{H}$ in the spectrometer. The pyrazole $4-\mathrm{H}$ is known to be acidic and therefore exchangable for other functions. 
The simulation of a species with one proton exchanged for NO $(m / z=495.1)$ is close to the measured value of $m / z=495.2$. This would also explain the charge of +2 . However, the low resolution of the ESI-spectrum does not permit assumptions based on such small mass differences. In conclusion, it was possible to generate a new species when $\mathrm{L}^{3} \mathrm{Cu}_{2}^{\mathrm{I}} \mathrm{OTf}$

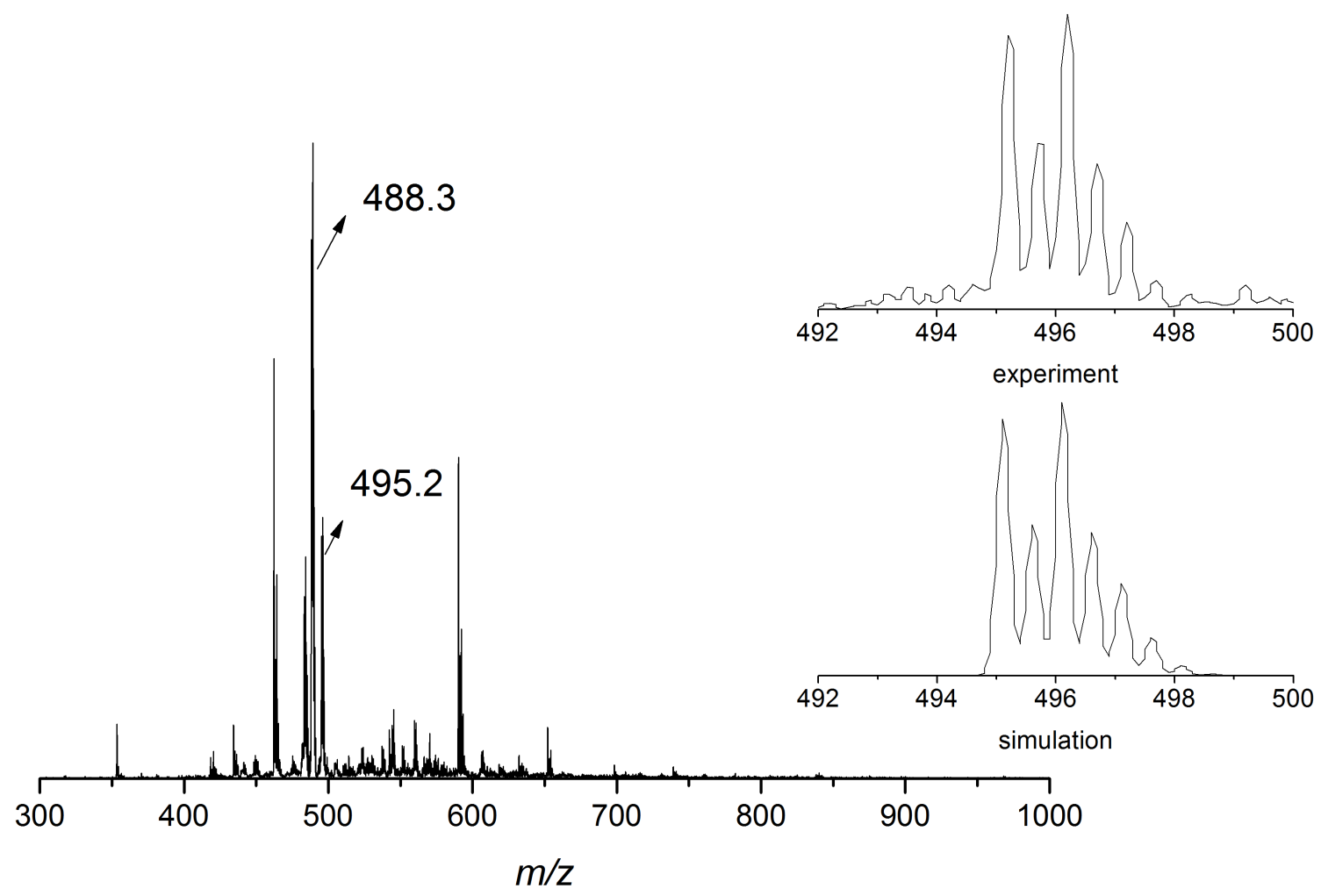

Figure 6.15 ESI mass spectrum of a solution of $\mathbf{9}$ and gaseous NO in acteonitrile. Inset shows enlargement and simulation of $\left.\left[\mathbf{L}^{3} \mathrm{Cu}_{2}(\mathrm{NO})_{2}\right)\right]^{2+}$.

was reacted with gaseous NO under inert conditions. UV/Vis spectroscopy indicates the formation of a new species, which was confirmed by IR spetroscopy. An IR spectrum of the solution of $\mathbf{9}$ and gaseous NO in MeCN showed two new peaks at 1088 and $1339 \mathrm{~cm}^{-1}$ that would fit to a hyponitrite species. A UV/Vis spectrum of $\mathbf{9}$ and gaseous NO in MeCN showed a new band at $675 \mathrm{~nm}$ with a small shoulder at $875 \mathrm{~nm}$ that coincide well with a spectrum of the copper/hyponitrite species (675 and $870 \mathrm{~nm}$ ) observed by KARLIN and coworkers. Unfortunately, ESI mass spectrometry provided no evidence for the assumption of a copper bound hyponitrite species.

\subsubsection{Reactivity with sodium phenolate}

The dicopper(II) complex was tested for phenol oxidation capabilities similar to those observed for $\mathbf{1}$ and $\mathbf{4}$ by means of UV/Vis spectroscopy. As the complex hosts no endogenous base, sodium 2,4,6-trimethyl phenolate was prepared in situ and added to a MeCN 
solution of 7 .

The reactions with one and two equivalents of TMP were run over night and the solution filtered through silica to remove the complex. The solvent was removed and the residue submitted for ${ }^{1} \mathrm{H}-\mathrm{NMR}$ analysis. The resulting spectra did not show significant differences and both indicated the presence of a mixture of many compounds.

Because a mixture of products and no clean conversion was observed, this aspect was not investigated further.

\subsubsection{Conclusion}

Complexes of copper(I) and copper(II) of the new ligand $\mathrm{HL}^{3}$ were synthesized and characterized succesfully. The oxidative coupling of NaTMP turned out to result in a mixture of compounds rather than a clean conversion. Copper(II) complexes of $\mathrm{HL}^{3}$ were reacted with different peroxides, sulfide and $\mathrm{Ph}_{3} \mathrm{CSNO}$ but showed no reactivity with these substrates. The reactivity of the copper(I) complexes with molecular oxygen, elemental sulfur and NO was investigated. However, no reactive intermediate for oxygen and sulfur was observed. A possible hyponitrite/copper(II) species was observed by UV/Vis and IR spectroscopy. However, it was not possible to verify the existece of the assumed species by ESI mass spectrometry. 


\section{Conclusion \& Outlook}

In the presented work, dicopper(I) and dicopper(II) complexes of four different compartmental pyrazolate bridged dinucleating ligands were prepared, fully characterized and investigated towards their capabilities to catalytically oxidize and functionalize phenolic substrates. The scope of bioinspired ligand systems bearing imidazole sidearms was expanded from the previously reported $\mathbf{H L}^{1}$ by two additional systems with $\mathbf{H L}^{3}$ and $\mathbf{H L}^{2}$.

The mechanism of the para-C-C coupling of 2,4,6-trimethylphenol to form TMBB and its subsequent oxidation to TMSQ catalyzed by $\mathbf{1}$ was elucidated by various spectroscopic methods. Furthermore, an intermediate mixed valent copper(I)-copper(II) species of the catalyst was isolated and structurally characterized.

The reaction conditions for the functionalization of TMP in para-position were optimized, the time trace of these reactions recorded and the mechanism as well as the involved catalyst species further investigated.

It was shown that for reactions catalyzed by the dicopper systems of $\mathbf{H L}^{1}$ and $\mathbf{H L}^{2}$ involving TMP, dioxygen is required as the terminal oxidant. The required amount of dioxygen per turnover was determined and thereby the fate of the oxygen molecule involved in the reoxidation of the reduced catalyst.

The polymerization reaction of 4-bromo-2,6-dimethyl phenol catalyzed by $\mathbf{1}$ was optimized, the mechanism was elucidated and the obatined polymer was fully characterized.

The direct synthesis of 4-hydroxy-3,5-dimethylbenzaldehyde was investigated and the involvement of a quinone methide intermediate discussed.

All these studies have shown the potential of bioinspired copper complexes bearing imidazole sidearms, based on biorelevant pyrazole ligands, to act as catalysts in various reactions involving the oxidation of phenols. Moreover, this work presents mechanistic insight, on the unusual oxidative $\mathrm{C}-\mathrm{C}$ coupling of TMP to yield TMSQ as well as the functionalization of TMP in para-position. These results will undoubtedly provide a significant background for the future advancement of studies on bioinspired phenol oxidation reactions. 



\section{Experimental section}

\subsection{General considerations}

The synthesis of building blocks, ligands or complexes that are sensitive to air or moisture was carried out employing the standard Schlenk technique or in an nitrogen filled glovebox. All glassware that was used has been dried at $120{ }^{\circ} \mathrm{C}$ overnight prior to use. Pirfication and drying of solvents was done according to established procedures. Prior to use, they were distilled and subsequently degassed by bubbling them with argon. Deuterium substituted solvents were first dried and then distilled the same way as their unsubstituted analogues. $\left[\mathrm{Cu}(\mathrm{MeCN})_{4}\right]^{+}$salts were synthesized from copper(I)oxide and the corresponding acids according to literature procedure. ${ }^{246}$ All other chemicals were purchased from Merck, Sigma Aldrich, abcr or VWR and used without further purification.

3,5-bis(hydroxymethyl)pyrazole ${ }^{[178}$ and bis((1-methyl imidazolyl)-methyl)amine ${ }^{[179]}$ were synthesized according to literature procedure.

\section{NMR spectroscopy}

${ }^{1} \mathrm{H}$ and ${ }^{13} \mathrm{C}\left\{{ }^{1} \mathrm{H}\right\}$ NMR spectra were recorded on Bruker Avance $500 \mathrm{MHz}$ or $300 \mathrm{MHz}$ spectrometers. Chemical shifts are reported in ppm relative to residual proton signals of the solvents $\left(\mathrm{CDCl}_{3}=7.26 \mathrm{ppm}, \mathrm{CD}_{3} \mathrm{CN}=1.94 \mathrm{ppm}, \mathrm{CD}_{3} \mathrm{OD}=3.31 \mathrm{ppm}\right.$, DMSO- $\mathrm{d}^{6}=$ $2.50 \mathrm{ppm})$.

\section{Mass spectrometry}

Low resolution ESI mass spectra under inert conditions were recorded on a Bruker (HCT Ultra) spectrometer coupled to a glovebox. MALDI-MS spectra were recorded on a Bruker Daltonics spectrometer.

\section{$U V /$ Vis spectroscopy}

$\mathrm{UV} /$ vis spectra, recorded at ambient temperature, were measured with a Cary 5000 Bio spectrophotometer using quartz cuvettes that were sealed by a rubber septum. Low temperature UV-vis spectra were either recorded with a Varian Cary 50 Bioinstrument coupled to a quartz immersion probe (1 mm, Hellma Analytics), or on an Agilent Cary 60 equipped with an Unisoku Cryostat (CoolSpek) and magnetic stirrer using quartz cuvettes with attached tube and screw cap with septum.

Magnetic measurements 
Temperature-dependent magnetic susceptibility measurements were carried out using a Quantum-Design MPMS XL-5 SQUID magnetometer equipped with a $5 \mathrm{~T}$ magnet with a variable temperature ranging from $300 \mathrm{~K}$ to $2 \mathrm{~K}$. The polycrystalline sample was placed in a capsule (made of gelatin), if necessary covered with polyfluorinated oil, and placed in a non-magnetic sample holder. Correction for the diamagnetic contribution of the gelatin capsule was applied to the raw data according to $M^{\text {dia }}($ capsule $)=\chi \mathrm{g} \cdot \mathrm{m} \cdot \mathrm{H}$, with a gram susceptibility of the gelatin capsule, that was determined experimentally. Data of molar susceptibility was corrected for the diamagnetic contribution using the increment method according to Haberditzl as well as the Pascal constants. The experimental data was simulated with full matrix diagonalization of exchange coupling using the $j u l X$ program (E. Bill, Max-Planck Institute for Chemical Energy Conversion, Mülheim/Ruhr, Germany, 2008). The influence of temperature independent paramagnetism $(T I P)$ as well as paramagnetic impurity $(P I)$ were considered according to:

$\chi_{\text {calcd }}=\chi \cdot(1-P I)+\chi m o n o \cdot P I+T I P$

The coupling constant $J_{\mathrm{AB}}$ was obtained by using the Heisenberg-Dirac-vanVleck (HDvV) Hamiltonian

$H_{\mathrm{HDvV}}=-2 J_{\mathrm{AB}} \cdot S_{A} \cdot S_{B}$

\section{Resonance Raman spectroscopy}

A LabRAM HR Vis $(400-1100 \mathrm{~nm})$ with an open-electrode CCD detector was used to record Resonance Raman spectra. An $\mathrm{Ar}^{+}$ion laser with a wavelength of 488.7 $\mathrm{nm}$, was chosen because it coincides with the intense absorption transition of the copper(II)-phenolate species. Measurement of solids was carried out at room temperature. Measurements in frozen solution were carried out in liquid nitrogen.

\section{EPR spectroscopy}

EPR measurements were performed on a Bruker ELEXSYS E 500 continuous wave spectrometer (CW EPR) using X-band radiation. Cooling was provided using either liquid nitrogen or liquid helium. Spectrum simulation with easyspin afforded the calculated Spin Hamiltonian parameters. 247.

\section{Oxygen Uptake Experiments}

Oxygen uptake experiments were performed in a self-made glass reactor (each $\mathrm{V}=$ $16.5 \mathrm{~mL}$ ) whose temperature was controlled at $25^{\circ} \mathrm{C}$. To monitor the increasing oxygen concentration, a Clark oxygen sensor (UNISENSE, Ox-N sensor and Oxy-meter) was placed in the gas phase of the reactor. The oxygen sensor had to be calibrated prior to the experiment. Therefore, $2 \mathrm{~mL}$ of degassed MeCN were added and defined amounts of dioxygen were added to the degassed reactor. The complex was added to the degassed 
reactor in $1.5 \mathrm{~mL} \mathrm{MeCN}$. When the dioxygen value measured in the reactor was stable, the substrate was added in $0.5 \mathrm{~mL} \mathrm{MeCN}$ to the reactor.

\section{Binding constants}

Binding constants of phenolates to the corresponding dicopper complex were determined using an equation for a high affinity binding equilibrium derived from enzyme kinetics. 186 . A 1:1 binding ratio of complex $([\mathrm{C}])$ and substrate $([\mathrm{S}])$ was assumed and the following equation was fitted to a plot of the absorbtion difference $(\Delta \mathrm{A})$ of the unsubstituted complex.

$[\mathrm{S}]+[\mathrm{C}] \rightleftarrows[\mathrm{CS}]$

$\Delta A=\frac{\Delta A_{\infty}}{2 K[\mathrm{C}]_{0}}=\left\{K\left([\mathrm{C}]_{0}+[\mathrm{S}]_{0}\right)+1-\sqrt{\left.\left.K^{2}(\mathrm{C}]_{0}-[\mathrm{S}]_{0}\right)^{2}+2 k(\mathrm{C}]_{0}+[\mathrm{S}]_{0}\right)+1}\right\}$

$\Delta A_{\infty}$ is the absorbance change at saturation, $[\mathrm{C}]_{0}$ the initial complex concentration, $[\mathrm{S}]_{0}$ the total substrate concentration $([\mathrm{S}]+[\mathrm{CS}])$ and $K$ the equilibrium constant.

\section{X-Ray Crystallography}

Data for x-ray structure determination were collected on a STOE IPDS II diffractometer equipped with an area detector (graphite monochromated MO-K $\alpha$ radiation, $\lambda=0.71073$ $\AA)$ employing $\omega$ scans at $133 \mathrm{~K}$. Solving of structures was done by using direct methods and refined against F 2 using all reflections with SHELX-2013.[172] Unless stated otherwise, anisotropic refinement was used for non-hydrogen atoms. Hydrogen atoms were positioned in calculated coordinates and assigned to an isotropic displacement parameter of 1.2/1.5 Ueq $(\mathrm{C})$ or 1.5 Ueq. Corrections for face-indexed absorption was performed by use of X-RED (STOE \& CIE GmbH, Darmstadt, Germany, 2002). Additional details on crystal data and refinement can be found in the Appendix.

\subsection{Synthesis of bulding blocks and Ligands}

\subsubsection{Synthesis of 3,5-Bis(chloromethyl)-1-(tetrahydropyran-2- yl)-pyrazole}

\section{3,5-Bis(chloromethyl)pyrazole}

The synthesis was carried out according to literature procedure. 178

The product was obtained as colourless crystalline material.

yield: $4.35 \mathrm{~g}, 21.6 \mathrm{mmol}, 89 \%$.

${ }^{1} \mathbf{H}$ NMR $\left(300 \mathrm{MHz}, \mathrm{DMSO} \mathrm{d}^{6}\right): \delta(\mathrm{ppm})=4.72\left(\mathrm{~s}, 4 \mathrm{H}, \mathrm{CH}_{2}\right), 6.39(\mathrm{~s}, 1 \mathrm{H}, \mathrm{CHpz})$, $7.94\left(\mathrm{~s}_{b r}, 2 \mathrm{H}, \mathrm{NH}\right)$.

${ }^{13} \mathbf{C}\left\{{ }^{1} \mathbf{H}\right\}$ NMR $\left(75 \mathrm{MHz}, \mathrm{DMSO} \mathrm{d}^{6}\right): \delta(\mathrm{ppm})=37.3\left(\mathrm{CH}_{2}\right), 105.1(4-\mathrm{CHpz}), 144.1$ (3,5-Cpz). 


\section{3,5-Bis(chloromethyl)-1-(tetrahydropyran-2-yl)-pyrazole}

3,5-Bis(chloromethyl)pyrazole hydrochloride $(2.000 \mathrm{~g}, 8.18 \mathrm{mmol})$ was suspended in DCM $(25 \mathrm{~mL})$ and 3,4-dihydro-2 $H$-pyrane $(2.75 \mathrm{~mL}, 30.4 \mathrm{mmol})$ was added. The resulting solution was stirred at room temperature for $1 \mathrm{~d}$ and subsequently hydrolyzed by addition of sodium carbonate in water $(1.5 \mathrm{M}, 30 \mathrm{~mL})$. The organic phase was separated and dried over $\mathrm{MgSO}_{4}$. Volatile components were removed in vacuo and petroleum ether was added to the residue. The precipitate was recrystallized from DCM and hexane which gave the product as colourless needles.

yield: $1.56 \mathrm{~g}, 6.26 \mathrm{mmol}, 76 \%$.

${ }^{1} \mathbf{H}$ NMR $\left(300 \mathrm{MHz}, \mathrm{CDCl}_{3}\right): \delta(\mathrm{ppm})=1.60-1.78\left(\mathrm{~m}, 3 \mathrm{H}, \mathrm{CH}_{2}{ }^{4^{\prime}}, \mathrm{CH}_{2}{ }^{5^{\prime}}\right), 2.00-2.15$ $\left(\mathrm{m}, 2 \mathrm{H}, \mathrm{CH}_{2}{ }^{3^{\prime}}, \mathrm{CH}_{2}{ }^{4^{\prime}}\right), 2.33-2.48\left(\mathrm{~m}, 1 \mathrm{H} \mathrm{CH}_{2}{ }^{3^{\prime}}\right), 3.66-3.75\left(\mathrm{~m}, 1 \mathrm{H}, \mathrm{CH}_{2}{ }^{6^{\prime}}\right), 4.02-4.08$ $\left(\mathrm{m}, 1 \mathrm{H}, \mathrm{CH}_{2}{ }^{6^{\prime}}\right), 4.59\left(2,2 \mathrm{H}, \mathrm{ClCH}_{2}{ }^{3}\right), 4.67$ (s, $\left.1 \mathrm{H}, \mathrm{ClCH}_{2}{ }^{5}\right), 4.70\left(\mathrm{~s}, 1 \mathrm{H}, \mathrm{ClCH}_{2}{ }^{5}\right)$, $5.47\left(\mathrm{dd},{ }^{3} \mathrm{~J}=9.6,2.6 \mathrm{~Hz}, 1 \mathrm{H}, \mathrm{CH}-2\right), 6.42\left(\mathrm{~s}, 1 \mathrm{H}, \mathrm{CH}^{4}\right)$.

\subsubsection{Synthesis of bis(2-(1-methylbenzimidazolyl)ethyl)amine}

The synthesis was carried out according to literature procedure. 248 Instead of N-methylortho-phenylene diamine, its dihydrochloride salt was used.

yield: $1.067 \mathrm{~g}, 3.2 \mathrm{mmol}, 25 \%$.

${ }^{1} \mathbf{H}$ NMR $\left(300 \mathrm{MHz}, \mathrm{CDCl}_{3}\right): \delta(\mathrm{ppm})=2,44\left(\mathrm{~s}_{b r}, \mathrm{NH}, 1 \mathrm{H}\right), 3.27(\mathrm{t}, J=6.4 \mathrm{~Hz}$, $\left.\mathrm{NCH}_{2} \mathrm{CH}_{2}, 4 \mathrm{H}\right), 3.27$ (t, $\left.J=6.4 \mathrm{~Hz}, \mathrm{NCH}_{2}, 4 \mathrm{H}\right), 3.71$ (s, $\left.\mathrm{CH}_{3}, 6 \mathrm{H}\right), 7.20-7.32$ (m, Ar-H, $6 \mathrm{H})$, 7.56-7.62 (m, Ar-H, 2H).

${ }^{13} \mathbf{C}\left\{{ }^{1} \mathbf{H}\right\} \mathbf{N M R}\left(75 \mathrm{MHz}, \mathrm{CDCl}_{3}\right): \delta(\mathrm{ppm})=27.3 \mathrm{NCH}_{2} \mathrm{CH}_{2}, 29.8\left(\mathrm{NCH}_{3}\right), 46.8$ $\left(\mathrm{NCH}_{2}\right), 109.2$ (Ar-C), 119.3 (Ar-C), 122.1 (Ar-C), 122.4 (Ar-C), $135.8 / 5$-C ${ }^{\mathrm{im}}$ ), 142.4 $\left(4-\mathrm{C}^{\mathrm{im}}\right), 153.1\left(2-\mathrm{C}^{\mathrm{im}}\right)$.

\subsubsection{Synthesis of Bis(1-methyl-2-phenyl-4- imidazolylmethyl)amine}

\section{(2-Phenyl-1H-imidazol-4-yl)methanol}

The synthesis is based on a modified literature procedure. 249]

Benzamidine hydrochloride $(10.168 \mathrm{~g}, 64.9 \mathrm{mmol})$, dihydroxy aceton dimer $(11.697 \mathrm{~g}$, $64.9 \mathrm{mmol}$ ) and ammonium chloride $(13.553 \mathrm{~g}, 253.3 \mathrm{mmol})$ were dissolved in ammonium hydroxide solution $(25 \%)$ and heated to $80^{\circ} \mathrm{C}$ for $30 \mathrm{~min}$. The mixture was let cool down and stored at $4{ }^{\circ} \mathrm{C}$ over night. The precipitate was filtered off, washed with diluted ammonia solution and dried in vacuo. The product was obtained as an off white solid. 
yield: $6.228 \mathrm{~g}, 35.8 \mathrm{mmol} 55 \%$.

${ }^{1} \mathbf{H}$ NMR $\left(300 \mathrm{MHz}, \mathrm{DMSO}-\mathrm{d}^{6}\right): \delta(\mathrm{ppm})=4.44\left(\mathrm{~s}, \mathrm{CH}_{2}, 2 \mathrm{H}\right), 5.01\left(\mathrm{~s}_{b r}, \mathrm{OH}, 1 \mathrm{H}\right), 6.98$ (s, H-5, 1H), 7.23-7.35 (m, Ar-H, 1H), 7.37-7.48 (m, Ar-H, 2H), 7.87-7.99 (m, Ar-H, 2H), $12.44\left(\mathrm{~s}_{b r}, \mathrm{NH}, 1 \mathrm{H}\right)$

${ }^{13} \mathbf{C}\left\{{ }^{1} \mathbf{H}\right\}$ NMR $\left(75 \mathrm{MHz}, \mathrm{DMSO} \mathrm{d}^{6}\right): \delta(\mathrm{ppm})=55.8\left(\mathrm{CH}_{2} \mathrm{OH}\right), 124.7(\mathrm{Ar}-\mathrm{CH}), 127.8$, $128.7,128.9,130.9(2-\mathrm{C})$.

\section{2-Phenyl-1H-imidazole-4-carbaldehyde}

2-Phenyl-1 $H$-imidazol-4-yl)methanol $(6.200 \mathrm{~g}, 35.6 \mathrm{mmol})$ was dissolved in THF and $\mathrm{MnO}_{2}(30.943 \mathrm{~g}, 356 \mathrm{mmol})$ was added. The mixture was heated to $80{ }^{\circ} \mathrm{C}$ for $3 \mathrm{~h}$ and let cool to remtemperature. The mixture was filtered through celite and the solvent removed in vacuo. The product was obtained as a yellow-orange solid.

yield: $5.510 \mathrm{~g}, 32.0 \mathrm{mmol}, 90 \%$.

${ }^{1} \mathbf{H}$ NMR $\left(300 \mathrm{MHz}, \mathrm{DMSO}-\mathrm{d}^{6}\right): \delta(\mathrm{ppm})=7.34-7.66(\mathrm{~m}, \mathrm{Ar}-\mathrm{H}, 3 \mathrm{H}), 7.99-8.09(\mathrm{~m}$, Ar-H, 2H), 8.11 (s,5-H, 1H), 9.76 (s, C(O)H, 1H), $13.44\left(\mathrm{~s}_{b r}, \mathrm{NH}, 1 \mathrm{H}\right)$.

${ }^{13} \mathbf{C}\left\{{ }^{1} \mathbf{H}\right\}$ NMR $\left(75 \mathrm{MHz}, \mathrm{DMSO} \mathrm{d}^{6}\right): \delta(\mathrm{ppm})=125.5,125.8,127.5,128.2$ 128.9, 129.4, $131, .2,184.8$ (CHO).

\section{1-Methyl-2-phenyl-4-formylimidazole}

The synthesis is based on a modified literature procedure. 181

To a suspension of NaH $(1.279 \mathrm{~g}, 32.0 \mathrm{mmol})$ in dry THF ( $80 \mathrm{~mL})$ 2-Phenyl-1H-imidazole4-carbaldehyde $(5.500 \mathrm{~g}, 32.0 \mathrm{mmol})$ was added in portions. When gas evolution ceased, MeI $(5.450 \mathrm{~g}, 38.4 \mathrm{mmol})$ was added and the mixture left stirring over night. The mixture was filtered, the solvent removed and the reisude taken up in $\mathrm{H}_{2} \mathrm{O}$. The aqueous phase was extracted with ethyl acetate $(3 \cdot 30 \mathrm{~mL})$, the combined organic phases dried over $\mathrm{NaSO}_{4}$ and the solvent removed in vacuo. The residue was purified by column chromatography (Silica, EtOAC/Hexane $1 / 1 \rightarrow$ EtOAC).

yield: $5.362 \mathrm{~g}, 28.8 \mathrm{mmol}, 90 \%$.

${ }^{1} \mathbf{H}$ NMR $\left(300 \mathrm{MHz}, \mathrm{CDCl}_{3}\right): \delta(\mathrm{ppm})=3.82\left(\mathrm{~s}, \mathrm{CH}_{3}, 3 \mathrm{H}\right), 7.47-7.85(\mathrm{~m}, \mathrm{Ar}-\mathrm{H}, 3 \mathrm{H})$, 7.69-7.77 (m, Ar-H, 2H), 8.18 (s, 5- H, 1H), 9.76 (s, C(O)H, 1H).

${ }^{13} \mathbf{C}\left\{{ }^{1} \mathbf{H}\right\}$ NMR $\left(75 \mathrm{MHz}, \mathrm{CDCl}_{3}\right): \delta(\mathrm{ppm})=35.3\left(\mathrm{NCH}_{3}\right), 127.6$ ( $p$-C ophenyl), 128.9 ( $m$-C pheny), 129.0 (o-C phenyl), 129.4 (4-C midazole), 129.8 (5-C imidazole), 141.22 (2-C imidazole), 149.8 (1-C phenyl), 186.4 (CHO).

\section{Bis(1-methyl-2-phenyl-4-imidazolylmethyl)benzylamine}

The synthesis is based on a modified literature procedure. 181 1-Methyl-2-phenyl4-formylimidazole $(2.500 \mathrm{~g}, 13.4 \mathrm{mmol})$ and benzylamine $(1.438 \mathrm{~g}, 13.4 \mathrm{mmol})$ were dissolved in THF $(80 \mathrm{~mL})$. $\mathrm{NaBH}(\mathrm{OAc})_{3}(8.520 \mathrm{~g}, 40.2 \mathrm{mmol})$ was added and the mixture 
stirred over night. Acetic acid $(2.414 \mathrm{~g}, 40.2 \mathrm{mmol}$ ) and another equivalent of the aldehyde $(2.500 \mathrm{~g}, 13.4 \mathrm{mmol})$ were added and the mixture stirred for $8 \mathrm{~h}$. Aqueous $\mathrm{NaOH}$ solution was added until the solution was basic. The solvent was removed and $\mathrm{NaCl}$ solution $(50 \mathrm{~mL})$ was added and the solution extracted with $\mathrm{CHCl}_{3}(3 \cdot 30 \mathrm{~mL})$. The combined extracts were dried over $\mathrm{MgSO}_{4}$ and the solvent removed in vacuo. The residue was purified by column chromatography (Alox (neutral), Hexane/ethyl acetate, $1 / 1)$.

yield:5.158 g, $115.2 \mathrm{mmol}, 86 \%$.

${ }^{1} \mathbf{H}$ NMR $\left(300 \mathrm{MHz}, \mathrm{CDCl}_{3}\right): \delta(\mathrm{ppm})=3.71\left(\mathrm{~s}, \mathrm{CH}_{3}, 6 \mathrm{H}\right), 3.79\left(\mathrm{~s}, \mathrm{CH}_{2}, 4 \mathrm{H}\right), 3.82(\mathrm{~s}$, Ph- $\left.\mathrm{CH}_{2}, 2 \mathrm{H}\right), 7.02$ (s, CH, 2H), 7.15-7.51 (m, Ar-H, 11H), 7.58-7.70 (m, 4H).

${ }^{13} \mathbf{C}\left\{{ }^{1} \mathbf{H}\right\} \quad$ NMR $\left(75 \mathrm{MHz}_{2} \mathrm{CDCl}_{3}\right): \quad \delta(\mathrm{ppm})=34.4\left(\mathrm{NCH}_{3}\right), 50.8\left(\mathrm{NCH}_{2}-\mathrm{Im}\right), 57.3$ $\left(\mathrm{NCH}_{2}-\mathrm{Bn}\right), 121.7$ (4-CH ${ }^{\mathrm{Im}}, 127.5$ (Ar-C), 128.2 (Ar-C), 128.4 (Ar-C), 128.5 (Ar-C), 128.7 (Ar-C), 128.7 (Ar-C), 129.3 (1-C $\left.{ }^{\mathrm{Bn}}\right), 130.26,137.35138 .1,147.3\left(2-\mathrm{C}^{\mathrm{Im}}\right)$.

\section{Bis(1-methyl-2-phenyl-4-imidazolylmethyl)amine}

The synthesis was carried out acording to literature procedure. ${ }^{181}$ The analytical properties were consistent with literature.

yield: $1.533 \mathrm{~g}, 4.29 \mathrm{mmol}, 96 \%$.

${ }^{1} \mathbf{H}$ NMR $\left(300 \mathrm{MHz}, \mathrm{CD}_{3} \mathrm{OD}\right): \delta(\mathrm{ppm})=3.98\left(\mathrm{~s}, \mathrm{CH}_{3}, 6 \mathrm{H}\right), 4.64\left(\mathrm{~s}, \mathrm{CH}_{2}, 4 \mathrm{H}\right), 7.64$ 7.80 (m, Ar-H, 6H), $7.83-7.89$ (m, Ar-H, 4H), 8.00 (s, CH, 2H).

${ }^{13} \mathbf{C}\left\{{ }^{1} \mathbf{H}\right\}$ NMR $\left(75 \mathrm{MHz}, \mathrm{CDCl}_{3}\right): \delta(\mathrm{ppm})=34.5\left(\mathrm{NCH}_{3}, 51.5\left(\mathrm{CH}_{2}\right.\right.$ imidazole $), 57.9$ (CH-2 benzyl), 120.8 (5-C imidazole), 126.7 ( $p$-C benzyle), 128.2 ( $m$-C benzyle), 128.5 ( $m, p$-C phenyl, 128.8 (o-C benzyl), 130.0 (o-C phenyl, 130.9 (4-C imidazole, 139.4 (1-C benzyl), 140.2 (2-C imidazole), 147.1 (1-C phenyl).

\subsubsection{Synthesis of $\mathrm{HL}^{1}$}

The synthesis was carried out according to literature procedure. 250 yield: $20.845 \mathrm{~g}, 41.5 \mathrm{mmol}, 83 \%$.

${ }^{1} \mathbf{H}$ NMR $\left(300 \mathrm{MHz}, \mathrm{CDCl}_{3}\right): \delta(\mathrm{ppm})=3.45\left(\mathrm{~s}, \mathrm{CH}_{3}, 12 \mathrm{H}\right), 3.57\left(\mathrm{~s}, \mathrm{pzCH}_{2}, 4 \mathrm{H}\right), 3.62$ $\left(\mathrm{s}, \mathrm{NCH}_{2}, 8 \mathrm{H}\right), 6.14(\mathrm{~s}, \mathrm{pz}-\mathrm{CH}, 1 \mathrm{H}), 6.79(\mathrm{~d}, J=1 \mathrm{~Hz}, \mathrm{im}-\mathrm{CH}, 4 \mathrm{H}), 6.91(\mathrm{~d}, J=1 \mathrm{~Hz}$, im- $\mathrm{CH}, 4 \mathrm{H})$.

${ }^{13} \mathbf{C}\left\{{ }^{1} \mathbf{H}\right\} \mathbf{N M R}\left(75 \mathrm{MHz}, \mathrm{CDCl}_{3}\right): \delta(\mathrm{ppm})=32.4\left(\mathrm{CH}_{3}\right), 48.6\left(\mathrm{CH}_{2}\right), 49.0\left(\mathrm{CH}_{2}\right), 106.7$ $\left(4 \mathrm{C}^{\mathrm{pz}}\right), 121.4$ (im-C), 127.0 (im-C), 145.0 (im-C2), 3,5- $\mathrm{C}^{\mathrm{pz}}$ was not observed. 


\subsubsection{Synthesis of $\mathrm{HL}^{2}$}

Bis(2-(1-methylbenzimidazolyl)ethyl)amine (1.326 g, $3.98 \mathrm{mmol})$, sodium carbonate (5.835 g, $55.05 \mathrm{mmol})$, tetrabutyl ammonium bromide $(0.129 \mathrm{~g}, 0.40 \mathrm{mmol})$ and 3,5Bis(chloromethyl)-1-(tetrahydropyran-2-yl)-pyrazole (0.495 g, $1.99 \mathrm{mmol}$ ) were dissolved in dry THF under argon atmosphere and heated to $60{ }^{\circ} \mathrm{C}$ for 3 days. The reaction mixture was filtered, the solvent removed in vacuo and the residue purified by column chromatography (Alox (neutral), Acetone to eluate pink side product, 2. DCM /w $0.75 \% \mathrm{MeOH}$ ). The obtained yellow solid was dissolved in ethanolic $\mathrm{HCl}(6.4 \mathrm{M})$ and stirred over night at roomtempeature. $\mathrm{Et}_{2} \mathrm{O}$ was added and the precipitate was filtered off, suspended in aqueous $\mathrm{NaOH}$ solution $(1.5 \mathrm{M}, 20 \mathrm{~mL})$. The aqueous phase was extracted with DCM (5 $\cdot 20 \mathrm{~mL}$ ), the combined organic phases dried over $\mathrm{Na}_{2} \mathrm{SO}_{4}$, filtered and the solvent removed in vacuo. $\mathrm{HL}^{3}$ was obtained as a yellow solid.

yield: $1.172 \mathrm{~g}, 1.45 \mathrm{mmol}, 73 \%$.

${ }^{1} \mathbf{H}$ NMR $\left.\left(300 \mathrm{MHz}, \mathrm{CDCl}_{3}\right): \delta(\mathrm{ppm})=\right) 3.70\left(\mathrm{~s}, 24 \mathrm{H}, \mathrm{NCH}_{3}\right), 3.74\left(\mathrm{~s}, 8 \mathrm{H}, \mathrm{CH}_{2}\right), 3.84$ ( $\mathrm{s}, 4 \mathrm{H}, \mathrm{CH}_{2}\left(3,5\right.$ pyrazole), 6.35 (s, $1 \mathrm{H}, 4-\mathrm{H}$ pyrazole, 7.05 ( $\mathrm{s}_{b r}, 4 \mathrm{H}, 4-\mathrm{H}$ (imidazole), 7.34 - 7.47 (m, 12 H, 3,4,5-Phenyl), 7.61 - 7.65 (m, 8 H, 2,6-H phenyl).

${ }^{13} \mathbf{C}\left\{{ }^{1} \mathbf{H}\right\} \mathbf{N M R}\left(75 \mathrm{MHz}, \mathrm{CDCl}_{3}\right): \delta(\mathrm{ppm})=34.56\left(\mathrm{NCH}_{3}\right), 50.84\left(\mathrm{NCH}_{2}, \mathrm{CH}_{2}^{\mathrm{pz}}\right), 121.54$ $\left(5-\mathrm{C}^{\mathrm{Im}}\right), 128.54 \& 128.74\left(2-\mathrm{C}^{\mathrm{Ph}}, 6-\mathrm{C}^{\mathrm{Ph}}\right), 128.85\left(3-\mathrm{C}^{\mathrm{Ph}}, 5-\mathrm{C}^{\mathrm{Ph}}\right), 130.92\left(1-\mathrm{C}^{\mathrm{Ph}}\right), 131.10$ $\left(4-\mathrm{C}^{\mathrm{Ph}}\right), 147.09\left(3,5-\mathrm{C}^{\mathrm{pz}}\right), 156.76\left(2-\mathrm{C}^{\mathrm{Im}}\right)$. The pyrazole $4-\mathrm{C}$ was not visible in the spectrum.

\subsubsection{Synthesis of $\mathrm{HL}^{3}$}

Bis(1-methyl-2-phenyl-4-imidazolylmethyl)amine $\quad(2.000 \mathrm{~g}, \quad 5.60 \mathrm{mmol}), \quad$ 3,5Bis(chloromethyl)-1-(tetrahydropyran-2-yl)-pyrazole $\quad(0.697 \mathrm{~g}, \quad 2.80 \mathrm{mmol}), \quad$ sodium carbonate $(2.965 \mathrm{~g}, 27.80 \mathrm{mmol})$ and tetrabutyle ammonium bromide (0.090 g, $0.28 \mathrm{mmol})$ were dissolved in dry MeCN $(50 \mathrm{~mL})$ in an argon atmosphere and stirred at $60^{\circ}$ for $24 \mathrm{~h}$. After cooling to roomtemperature the mxture was filtered and the solvent removed under reduced pressure. The residue was purified by column chromatigraphy (Alox (neutral), EtOAc/Acetone $3 / 1 \rightarrow$ Acetone). The obtained compound was dissolved in ethanolic $\mathrm{HCl}(6.4 \mathrm{M}, 20 \mathrm{~mL})$ and stirred at roomtemperature overnight. Dietylether $(30 \mathrm{~mL})$ was added and the resulting precipate was collected by filtration. It was suspended in aqueos sodium hydroxide solution $(1 \mathrm{M}, 15 \mathrm{~mL})$ and extracted with $\mathrm{CH}_{2} \mathrm{Cl}_{2}(5 \cdot 15 \mathrm{~mL})$. The combined organic phases were dried over $\mathrm{Na}_{2} \mathrm{SO}_{4}$, filtered and the solvent was removed in vacuo to give $\mathrm{HL}^{4}$ as an off white solid.

yield: $0.964 \mathrm{~g} 1.19 \mathrm{mmol}, 60 \%$

${ }^{1} \mathbf{H}$ NMR $\left(300 \mathrm{MHz}, \mathrm{CDCl}_{3}\right): \delta(\mathrm{ppm})=2.93\left(8 \mathrm{H}, \mathrm{br}, \mathrm{CH}_{2}\right), 3.12-3.10\left(12 \mathrm{H}, \mathrm{m}, \mathrm{CH}_{3}\right)$, $3.36\left(8 \mathrm{H}, \mathrm{br}, \mathrm{CH}_{2}\right), 3.95\left(4 \mathrm{H}, \mathrm{s}\right.$, pyrazole- $\left.\mathrm{CH}_{2}\right), 6.00$ (1H, s, pyrazole- $\mathrm{H}$ ), $6.88-7.08$ 
(12H, m, br, imidazole-H) 7.71 (4H, d, $J=7.9 \mathrm{~Hz}$, benzimidazole-H).

${ }^{13} \mathbf{C}\left\{{ }^{1} \mathbf{H}\right\}$ NMR $\left(75 \mathrm{MHz}, \mathrm{CDCl}_{3}\right): \delta(\mathrm{ppm})=26.1$ (2- $\mathrm{CH}_{2}$ benzimidazole), $29.6\left(\mathrm{NCH}_{3}\right)$, 52.0(3,5- $\mathrm{CH}_{2}$ pyrazole), $52.4\left(\mathrm{NCH}_{2}\right), 102.5\left(4-\mathrm{C}^{\mathrm{pz}}\right), 109.3$ (Ar-C), 118.7 (Ar-C), 121.8 (Ar-C), 122.0 (Ar-C), 135.3 (5-C benzimidazole), 141.9 (3,5- $\mathrm{C}^{\mathrm{pz}}$ ), 154.0 (2-C benzimidazole).

\subsection{Synthesis of complexes}

\subsubsection{Synthesis of $\left[\mathrm{L}^{1} \mathrm{Cu}_{2}(\mathrm{OMe})(\mathrm{HOMe})\right]\left(\mathrm{ClO}_{4}\right)_{2}(1)$}

The synthesis was carried out according to a modified literature procedure. Instead of $\mathrm{Cu}\left(\mathrm{NO}_{3}\right)_{2} \cdot 3 \mathrm{H}_{2} \mathrm{O}, \mathrm{Cu}\left(\mathrm{ClO}_{4}\right) \cdot 6 \mathrm{H}_{2} \mathrm{O}$ was used. yield: $223 \mathrm{mg}, 0.24 \mathrm{mmol}, 60 \%$.

$\operatorname{MS}(\mathrm{ESI}(+), \mathrm{MeCN}): m / z=313.6[\mathrm{M}-\mathrm{OMe}-\mathrm{HOMe}]^{2+}$,

$\operatorname{IR}(\mathrm{KBr}): \quad \tilde{\nu}\left[\mathrm{cm}^{-1}\right]=3458(\mathrm{~s}), 3132(\mathrm{w}), 1555(\mathrm{~m}), 1509(\mathrm{w}), 1454(\mathrm{w}), 1381(\mathrm{w} 1286$ (w), $1086(\mathrm{vs}), 953(\mathrm{w}), 871(\mathrm{w}), 665(\mathrm{w}), 626(\mathrm{~m})$

UV/Vis (MeCN): $\lambda_{\max }\left(\epsilon\left[\mathrm{L} \cdot \mathrm{mol} \cdot \mathrm{cm}^{-1}\right): 730(135), 962(269)\right.$.

Elemental analysis calcd. (\%) for $\mathrm{C}_{27} \mathrm{H}_{40} \mathrm{Cu}_{2} \mathrm{~N}_{12} \mathrm{O}_{10} \mathrm{Cl}_{2} \cdot \mathrm{MeCN}$ (found): $\mathrm{C} 34.80$ (34.82), H 4.29 (4.27), N 18.04 (18.69).

\subsubsection{Synthesis of $\left[\mathrm{L}^{1} \mathrm{Cu}^{\mathrm{II}} \mathrm{Cu}^{\mathrm{I}}(\mathrm{MeCN})\left(\mathrm{ClO}_{4}\right)_{2}\right]_{2}(2)$}

To a solution of $\mathbf{1}$ in MeCN in a glovebox, 2,4,6-trimethylphenol was added and the mixture left stirring over night. The solution was layered with $\mathrm{Et}_{2} \mathrm{O}$ to give green single crystals, suitable for x-ray diffraction over several days. The obtained crystals were unsolluble. No further analysis was done.

\subsubsection{Synthesis of $\left[\mathrm{L}^{1} \mathrm{Cu}_{2}\left(\mathrm{O}_{2} \mathrm{C}_{9} \mathrm{H}_{9}\right)(\mathrm{HOMe})\right]\left(\mathrm{ClO}_{4}\right)_{2}(3)$}

A solution of $\mathrm{HL}^{1}(0.100 \mathrm{~g}, 0.20 \mathrm{mmol})$ in $\mathrm{MeOH}(20 \mathrm{~mL})$ was treated with two equivalents of $\mathrm{KO}^{t} \mathrm{Bu}(0.040 \mathrm{~g}, 0.40 \mathrm{mmol})$ and the solution was stirred for $60 \mathrm{~min}$. $\mathrm{Cu}\left(\mathrm{ClO}_{4}\right)_{2} \cdot 6 \mathrm{H}_{2} \mathrm{O}$ was added and stirring of the resulting green solution was continued over night at room temperature. After evaporation of all volatile material, the remaining green solid was dissolved in acetone/methanol (4/1) and 4-hydroxy-3,5-dimethyl-benzaldehyde (0.304 g, $0.2 \mathrm{mmol}$ ) was added. The solution was layered with $\mathrm{Et}_{2} \mathrm{O}$ and gave red, block shaped crystals after several days. Due to the small amount of obtained maerial, no further analysis was done. 


\subsubsection{Synthesis of $\left[\mathrm{L}^{2} \mathrm{Cu}_{2}\left(\mathrm{NO}_{3}\right)_{2}\right]\left(\mathrm{NO}_{3}\right)$}

A solution of $\mathrm{HL}^{2}(0.100 \mathrm{~g}, 0.13 \mathrm{mmol})$ in $\mathrm{MeOH}(15 \mathrm{~mL})$ was treated with one equivalent of $\mathrm{KO}^{t} \mathrm{Bu}(0.0016 \mathrm{~g}, 0.13 \mathrm{mmol})$ and the solution was stirred for $60 \mathrm{~min}$. $\mathrm{Cu}\left(\mathrm{NO}_{3}\right)_{2} \cdot 3 \mathrm{H}_{2} \mathrm{O}$ $(0.063 \mathrm{~g}, 0.26 \mathrm{mmol})$ was added and stirring of the resulting green solution was continued over night at room temperature. After evaporation of all volatile material, the remaining green solid was dissolved in $\mathrm{MeCN}$. Slow diffusion of $\mathrm{Et}_{2} \mathrm{O}$ into this solution led to the formation of green crystals of $4 \cdot 2 \mathrm{H}_{2} \mathrm{O}$.

yield: $45 \mathrm{mg}, 0.057 \mathrm{mmol} 44 \%$.

$\operatorname{MS}(\operatorname{ESI}(+), \mathrm{MeCN}): m / z=759.2[\mathrm{M}+\mathrm{H}]^{+}$.

$\operatorname{IR}(\mathrm{KBr}): \tilde{\nu}\left[\mathrm{cm}^{-1}\right]=3421(\mathrm{~m}), 3059(\mathrm{w}), 2942(\mathrm{~m}), 2850(\mathrm{w}), 1617(\mathrm{~m}), 1481(\mathrm{~m}), 1448$ (m), 1360 (vs), 1260 (m), 1238 (m), 1150 m), 1125 (m), 1099 (m), 1007 (m), 835 (w), 800 (w), $744(\mathrm{~s}), 568(\mathrm{w})$.

$\mathrm{UV} / \mathrm{V}$ is $(\mathrm{MeCN}): \lambda_{\max }\left(\epsilon\left[\mathrm{L} \cdot \mathrm{mol} \cdot \mathrm{cm}^{-1}\right): 670(198)\right.$.

Elemental analysis calcd. (\%) for $\mathrm{C}_{45} \mathrm{H}_{49} \mathrm{~N}_{12}\left(\mathrm{NO}_{3}\right)_{3} \mathrm{Cu}_{2}\left(\mathrm{H}_{2} \mathrm{O}\right)_{2}$ (found): $\mathrm{C} 48.82$ (48.79), H 4.83 (4.94), N 18.98 (19.31).

\subsubsection{Synthesis of $\left[\mathrm{L}^{2} \mathrm{Cu}_{2}\left(\mathrm{H}_{2} \mathrm{O}\right)(\mathrm{OH})\right]\left(\mathrm{NO}_{3}\right)_{2}(5)$}

A solution of $\mathrm{HL}^{2}(0.100 \mathrm{~g}, 0.13 \mathrm{mmol})$ in $\mathrm{MeOH}(15 \mathrm{~mL})$ was treated with two equivalents of $\mathrm{KO}^{t} \mathrm{Bu}(0.0032 \mathrm{~g}, 0.26 \mathrm{mmol})$ and the solution was stirred for $60 \mathrm{~min}$. $\mathrm{Cu}\left(\mathrm{NO}_{3}\right)_{2} \cdot 3 \mathrm{H}_{2} \mathrm{O}$ $(0.063 \mathrm{~g}, 0.26 \mathrm{mmol})$ was added and stirring of the resulting green solution was continued over night at room temperature. After evaporation of all volatile material, the remaining green solid was dissolved in MeCN. Slow diffusion of $\mathrm{Et}_{2} \mathrm{O}$ into this solution led to the formation of green crystals of $5 \cdot \mathrm{MeCN}$.

Due to the low amount of obtained material no further analysis was done.

\subsubsection{Synthesis of $\left[\mathrm{L}^{2} \mathrm{Cu}_{2}\right] \mathrm{OTf}(6)$}

A solution of $\mathrm{HL}^{2}(50 \mathrm{mg}, 0.066 \mathrm{mmol})$ in $\mathrm{MeCN}(5 \mathrm{~mL})$ was treated with one equivalent of $\mathrm{KO}^{t} \mathrm{Bu}(7 \mathrm{~m} \mathrm{~g}, 0.066 \mathrm{mmol})$ and the solution was stirred for $60 \mathrm{~min}$. $\left[\mathrm{Cu}(\mathrm{MeCN})_{4}\right] \mathrm{OTf}$ (50 $\mathrm{mg}, 0.132 \mathrm{mmol}$ ) was added and stirring of the resulting solution was continued over night at room temperature. The solution was layered with $\mathrm{Et}_{2} \mathrm{O}$ and gave a colourless powder after two days.

yield: $41 \mathrm{mg}, 0.040 \mathrm{mmol} 60 \%$.

$\operatorname{MS}(\operatorname{ESI}(+), \operatorname{MeCN}): m / z=833.3[\mathrm{M}]^{+}$.

${ }^{1} \mathbf{H}$ NMR $\left(300 \mathrm{MHz}, \mathrm{CDCl}_{3}\right): \delta(\mathrm{ppm})=2.83\left(\mathrm{~d}, J=13.5,8 \mathrm{H}, \mathrm{NCH}_{2} \mathrm{CH}_{2}\right), 2.94(\mathrm{~d}$, $\left.J=13.5,8 \mathrm{H}, \mathrm{NCH}_{2} \mathrm{CH}_{2}\right), 3.64\left(\mathrm{~s}, 12 \mathrm{H}, \mathrm{NCH}_{3}\right) 3.90\left(\mathrm{~s}, 4 \mathrm{H}, \mathrm{NCH}_{2}\right), 5.83\left(\mathrm{~s}, 1 \mathrm{H}, \mathrm{H}^{\mathrm{pz}}\right)$, $6.95(\operatorname{tr}, J=7.7 \mathrm{~Hz}, 4 \mathrm{H}, \mathrm{Ar}-\mathrm{H}), 7.16(\operatorname{tr}, J=7.7 \mathrm{~Hz}, 4 \mathrm{H}, \mathrm{Ar}-\mathrm{H}), 7.36(\mathrm{~d}, J=8.0 \mathrm{~Hz}, 4 \mathrm{H}$, 
Ar-H), 8.44 (d, $J=8.0 \mathrm{~Hz}, 4 \mathrm{H}, \mathrm{Ar}-\mathrm{H})$.

\subsubsection{Synthesis of $\mathrm{L}^{3} \mathrm{Cu}_{2}\left(\mathrm{NO}_{3}\right)_{3}(7)$}

A solution of $\mathrm{HL}^{3}(0.250 \mathrm{~g}, 0.31 \mathrm{mmol})$ in $\mathrm{MeOH}(15 \mathrm{~mL})$ was treated with one equivalent of $\mathrm{KO}^{t} \mathrm{Bu}(0.035 \mathrm{~g}, 0.31 \mathrm{mmol})$ and the solution was stirred for $60 \mathrm{~min} . \mathrm{Cu}\left(\mathrm{NO}_{3}\right)_{2} \cdot 3 \mathrm{H}_{2} \mathrm{O}$ $(0.150 \mathrm{~g}, 0.62 \mathrm{mmol})$ was added and stirring of the resulting green solution was continued over night at room temperature. After evaporation of all volatile material, the remaining green solid was dissolved in MeCN. Slow diffusion of $\mathrm{Et}_{2} \mathrm{O}$ into this solution led to the formation of a green precipitate.

yield: $0.278 \mathrm{~g}, 0.2 \mathrm{mmol}, 80 \%$.

$\operatorname{MS}(\operatorname{ESI}(+), \mathrm{MeCN}): m / z=481.1[\mathrm{M}+\mathrm{OMe}]^{2+}$.

IR(ATR): $\left.\left.\tilde{\nu}\left[\mathrm{cm}^{-1}\right]=745(\mathrm{~s}), 1010(\mathrm{~m}), 1280\right) \mathrm{s}\right), 1462(\mathrm{~m})$.

UV/Vis $(\mathrm{MeCN}): \lambda_{\max }\left(\epsilon\left[\mathrm{L} \cdot \mathrm{mol} \cdot \mathrm{cm}^{-1}\right): 730(141), 900(273)\right.$.

Elemental analysis calcd (\%) for $\mathrm{C}_{49} \mathrm{H}_{49} \mathrm{~N}_{15} \mathrm{Cu}_{2} \mathrm{O}_{9}$ (found): $\mathrm{C} 52.59$ (52.45), H 4.41 (4.34), N 18.77 (19.07).

\subsubsection{Synthesis of $\left[\mathrm{L}^{3} \mathrm{Cu}_{2}(\mathrm{OAc})\right]\left(\mathrm{NO}_{3}\right)_{2}$}

A solution of $\mathrm{HL}^{3}(0.250 \mathrm{~g}, 0.31 \mathrm{mmol})$ in $\mathrm{MeOH}(15 \mathrm{~mL})$ was treated with one equivalent of $\mathrm{KO}^{t} \mathrm{Bu}(0.035 \mathrm{~g}, 0.31 \mathrm{mmol})$ and the solution was stirred for $60 \mathrm{~min}$. $\mathrm{Cu}\left(\mathrm{ClO}_{4}\right)_{2} \cdot 6 \mathrm{H}_{2} \mathrm{O}$ $(0.110 \mathrm{~g}, 0.62 \mathrm{mmol})$ was added and stirring of the resulting green solution was continued over night at room temperature. After evaporation of all volatile material, the remaining green solid was dissolved in $\mathrm{MeCN} /$ chlorobenzene 9/1. Slow diffusion of $\mathrm{Et}_{2} \mathrm{O}$ into this solution led to the formation of a green precipitate after several weeks to give green blockshaped crystals of $\mathbf{8} \cdot 0.25\left(\mathrm{C}_{6} \mathrm{H}_{5} \mathrm{Cl}\right)$.

Due to the low amount of obtained material no further analysis was done.

\subsubsection{Synthesis of $\left[\mathrm{L}^{3} \mathrm{Cu}_{2}\right] \mathrm{OTf}(9)$}

A solution of $\mathrm{HL}^{3}(0.100 \mathrm{~g}, 0.12 \mathrm{mmol})$ in $\mathrm{MeCN}(5 \mathrm{~mL})$ was treated with one equivalent of $\mathrm{KO}^{t} \mathrm{Bu}(0.014 \mathrm{~g}, 0.12 \mathrm{mmol})$ and the solution was stirred for $60 \mathrm{~min}$. $\left[\mathrm{Cu}(\mathrm{MeCN})_{4}\right] \mathrm{OTf}$ $(0.090 \mathrm{~g}, 0.24 \mathrm{mmol})$ was added and stirring of the resulting solution was continued over night at room temperature. Slow diffusion of $\mathrm{Et}_{2} \mathrm{O}$ into this solution led to the formation of a white to yellowish precipitate over night to give $\mathbf{9}$.

yield: $0.084 \mathrm{~g}, 0.09 \mathrm{mmol}, 75 \%$.

$\operatorname{MS}(\operatorname{ESI}(+), \operatorname{MeCN}): m / z=466.3[\mathrm{M}+\mathrm{H}]^{2+}, 931.3[\mathrm{M}]^{+}$.

No useful NMR data could be obtained. 


\section{Bibliography}

[1] M. Tissot, H. Li, A. Alexakis in Copper-Catalyzed Asymmetric Syntesis, Wiley-VCH Verlag GmbH \& Co. KGaA, Weinheim, Germany, 2014, pp. 69-84.

[2] I. Garcia-Bosch, Synlett 2017, 28, 1237-1243.

[3] F. Nahra, Chim. Nouv. 2013, 114, 22-25.

[4] F. Lazreg, C. S. J. Cazin in N-Heterocyclic Carbenes, Wiley-VCH Verlag GmbH \\& Co. KGaA, Weinheim, Germany, 2014, pp. 199-242.

[5] K. Cao, J.-L. Wang, L.-H. Wang, Y.-Y. Li, X.-H. Yu, Y. Huang, J. Yang, G. Chang, Synth. Commun. 2014, 44, 2848-2853.

[6] S. E. Allen, R. R. Walvoord, R. Padilla-Salinas, M. C. Kozlowski, Chem. Rev. 2013, 113, 6234-6458.

[7] A. E. Wendlandt, A. M. Suess, S. S. Stahl, AC 2011, 123, 11256-11283.

[8] T. G. Moga, Nature Chem. 2012, 4, 334-334.

[9] E. I. Solomon, J. W. Ginsbach, D. E. Heppner, M. T. Kieber-Emmons, C. H. Kjaergaard, P. J. Smeets, L. Tian, J. S. Woertink, Faraday Discuss. 2011, 148 , 11-39; discussion 97-108.

[10] R. A. Festa, D. J. Thiele, Curr. Biol. 2011, 21, 877-883.

[11] R. L. Peterson, S. Kim, K. D. Karlin, Copper Enzymes, Vol. 3, Elsevier Ltd., 2013, pp. $149-177$.

[12] F. Monnier, M. Taillefer, AC 2009, 121, 7088-7105.

[13] J. K. Stille, ACIE 1986, 25, 508-524.

[14] V. Farina, S. Kapadia, B. Krishnan, C. Wang, L. S. Liebeskind, J. Org. Chem 1994, 59, 5905-5911.

[15] P. Espinet, A. M. Echavarren, ACIE 2004, 43, 4704-4734.

[16] J. Smidt, W. Hafner, R. Jira, R. Sieber, J. SedlMeier, A. Sabel, AC 1962, 1, 80-88.

[17] B. W. Michel, L. D. Steffens, M. S. Sigman in Org. React., John Wiley \& Sons, Inc., Hoboken, NJ, USA, 2014, pp. 75-414. 
[18] E. I. Solomon, D. E. Heppner, E. M. Johnston, J. W. Ginsbach, J. Cirera, M. Qayyum, M. T. Kieber-Emmons, C. H. Kjaergaard, R. G. Hadt, L. Tian, Chem. Rev. 2014, 114, 3659-3853.

[19] R. Balasubramanian, S. M. Smith, S. Rawat, L. A. Yatsunyk, T. L. Stemmler, A. C. Rosenzweig, Nature 2010, 465, 115-119.

[20] S. Sirajuddin, A. C. Rosenzweig, Biochemistry 2015, 54, 2283-2294.

[21] D. A. Armstrong, R. E. Huie, W. H. Koppenol, S. V. Lymar, G. Merenyi, P. Neta, B. Ruscic, D. M. Stanbury, S. Steenken, P. Wardman, Pure Appl. Chem. 2015, 87, 1139-1150.

[22] A. L. Feig, S. J. Lippard, Chem. Rev. 1994, 94, 759-805.

[23] I. A. Koval, P. Gamez, C. Belle, J. Reedijk, Chem. Soc. Rev. 2006, 35, 814-840.

[24] L. B. LaCroix, D. W. Randall, A. M. Nersissian, C. W. G. Hoitink, G. W. Canters, J. S. Valentine, E. I. Solomon, J. Am. Chem. Soc. 1998, 120, 9621-9631.

[25] E. I. Solomon, Inorg. Chem. 2006, 45, 8012-8025.

[26] R. L. Lieberman, D. M. Arciero, A. B. Hooper, A. C. Rosenzweig, Biochemistry 2001, 40, 5674-5681.

[27] E. I. Solomon, R. K. Szilagyi, S. D. George, L. Basumallick, Chem. Rev. 2004, 104, 419-458.

[28] J. P. Klinman, Chem. Rev. 1996, 96, 2541-2562.

[29] A. Gibbons, Science 2014, 346, 934-936.

[30] J.-S. Taylor, Science 2015, 31-35.

[31] E. Dadachova, R. A. Bryan, X. Huang, T. Moadel, A. D. Schweitzer, P. Aisen, J. D. Nosanchuk, A. Casadevall, PLoS One 2007, 2, 1-13.

[32] C. J. Coates, J. Nairn, Dev. Comp. Immunol. 2014, 45, 43-55.

[33] K. M. Lancaster, S. DeBeer George, K. Yokoyama, J. H. Richards, H. B. Gray, Nature Chem. 2009, 1, 711-715.

[34] A. C. Rosenzweig, Nature Chem. 2009, 1, 684-685.

[35] K. A. Magnus, B. Hazes, H. Ton-That, C. Bonaventura, J. Bonaventura, W. G. J. Hol, Proteins: Struct. Funct. Genet. 1994, 19, 302-309. 
[36] J. A. Halfen, S. Mahapatra, E. C. Wilkinson, S. Kaderli, V. G. Young, L. Que, A. D. Zuberbühler, W. B. Tolman, Science 1996, 271, 1397 - 1400.

[37] R. R. Jacobson, Z. Tyeklar, A. Farooq, K. D. Karlin, S. Liu, J. Zubieta, J. Am. Chem. Soc. 1988, 110, 3690-3692.

[38] K. E. Dalle, T. Gruene, S. Dechert, S. Demeshko, F. Meyer, J. Am. Chem. Soc. 2014, 2, 7428-7434.

[39] M. Becker, F. Heinemann, S. Schindler, Inorg. Chem. 1999, 42, 1800-6.

[40] M. Schatz, M. Becker, O. Walter, Inorg. Chim. Acta 2001, 324, 173-179.

[41] T. Hoppe, S. Schaub, J. Becker, C. Würtele, S. Schindler, ACIE 2013, 52, 870-873.

[42] L. M. Mirica, X. Ottenwaelder, T. D. P. Stack, Chem. Rev. 2004, 104, 1013-1045.

[43] N. Kindermann, E. Bill, S. Dechert, S. Demeshko, E. J. Reijerse, F. Meyer, ACIE 2015, 54, 1738-1743.

[44] N. Kitajima, K. Fujisawa, Y. Morooka, K. Toriumi, J. Am. Chem. Soc. 1989, 111, 8975-8976.

[45] E. I. Solomon, P. Chen, M. Metz, S.-K. Lee, A. E. Palmer, ACIE 2001, 40, 45704590.

[46] K. E. Van Holde, K. I. Miller, H. Decker, J. Biol. Chem. 2001, 276, 15563-15566.

[47] M. Goldfeder, M. Kanteev, S. Isaschar-ovdat, N. Adir, A. Fishman, Nat. Commun. 2014, 5, 1-5.

[48] E. Solem, F. Tuczek, H. Decker, ACIE 2016, 55, 2884-2888.

[49] T. Klabunde, C. Eicken, J. C. Sacchettini, B. Krebs, Nat. Struct. Biol. 1998, 5, 1084-1090.

[50] H. Decker, F. Tuczek, Trends Biochem. Sci. 2000, 25, 392-397.

[51] K. N. Naresh, A. Sreekumar, S. S. Rajan, J. Mol. Graph. Model. 2015, 61, 272-280.

[52] R. J. Deeth, C. Diedrich, J. Biol. Inorg. Chem. 2010, 15, 117-129.

[53] C. A. Ramsden, P. A. Riley, Bioorganic Med. Chem. 2014, 22, 2388-2395.

[54] A. W. J. W. Tepper, E. Lonardi, L. Bubacco, G. W. Canters in Handbook of Metalloproteins, Vol. 5, John Wiley \& Sons, Ltd, Chichester, UK, 2010, pp. 571-587. 
[55] M. Kanteev, M. Goldfeder, A. Fishman, Protein Sci. 2015, 24, 1360-1369.

[56] E. J. Land, C. Ramsden, P. Riley, Tohoku J. Exp. Med. 2007, 212, 341-348.

[57] A. C. Rosenzweig, P. Nordlund, P. M. Takahara, C. A. Frederick, S. J. Lippard, Chem. Biol. 1995, 2, 409-418.

[58] S. Sirajuddin, A. C. Rosenzweig, Biochemistry 2015, 54, 2283-2294.

[59] R. L. Lieberman, A. C. Rosenzweig, Nature 2005, 434, 177-182.

[60] M. A. Culpepper, G. E. Cutsail, W. A. Gunderson, B. M. Hoffman, A. C. Rosenzweig, J. Am. Chem. Soc. 2014, 136, 11767-11775.

[61] M. a. Culpepper, G. E. Cutsail, B. M. Hoffman, A. C. Rosenzweig, J. Am. Chem. Soc. 2012, 134, 7640-7643.

[62] J. S. Woertink, P. J. Smeets, M. H. Groothaert, M. a. Vance, B. F. Sels, R. A. Schoonheydt, E. I. Solomon, Proc. Natl. Acad. Sci. U. S. A. 2009, 106, 1890818913.

[63] L. Que, B. Tolman, William, Nature 2008, 455, 333-340.

[64] Y. Wang, Science 1998, 279, 537-540.

[65] P. Chaudhuri, M. Hess, T. Weyhermüller, K. Wieghardt, ACIE 1999, 38, 10951098.

[66] X. You, Z. Wei, H. Wang, D. Li, J. Liu, B. Xu, X. Liu, RSC Adv. 2014, 4, 6179061798.

[67] D.-W. Tan, J.-B. Xie, Q. Li, H.-X. Li, J.-C. Li, H.-Y. Li, J.-P. Lang, Dalton Trans. 2014, 43, 14061.

[68] H. Liu, M. Wang, H. Li, N. Luo, S. Xu, F. Wang, J. Catal. 2017, 346, 170-179.

[69] E. Article, Z. Huang, M. S. Askari, V. N. Esguerra, T.-y. Dai, Chem. Sci. 2016, 7, 358-369.

[70] W. Lu, H. Xu, Z. Shen, Org. Biomol. Chem. 2017, 15, 1261-1267.

[71] M. S. Askari, A. Proppe, B. Mcallister, J. Lumb, X. Ottenwaelder, Dalton Trans. 2015, 44, 12094-12097.

[72] T. V. Hansen, L. Skattebøl, Tetrahedron Lett. 2005, 46, 3357-3358. 
[73] A. Pezzella, L. Lista, A. Napolitano, M. D'Ischia, Tetrahedron Lett. 2005, 46, 35413544.

[74] C. Huang, N. Ghavtadze, B. Chattopadhyay, V. Gevorgyan, Synthesis 2011, 1763017633.

[75] S. Itoh, M. Ogino, S. Haranou, T. Terasaka, T. Ando, M. Komatsu, Y. Ohshiro, S. Fukuzumi, K. Kano, J. Am. Chem. Soc. 1995, 117, 1485-1493.

[76] B. Gigante, C. Santos, A. M. Silva, M. J. M. Curto, M. S. J. Nascimento, E. Pinto, M. Pedro, F. Cerqueira, M. M. Pinto, M. P. Duarte, A. Laires, J. Rueff, J. Gonçalves, M. I. Pegado, M. L. Valdeira, Bioorganic Med. Chem. 2003, 11, 1631-1638.

[77] C. Citek, C. T. Lyons, E. C. Wasinger, T. D. P. Stack, Nature Chem. 2012, 4, $317-322$.

[78] L. Santagostini, M. Gullotti, E. Monzani, L. Casella, R. Dillinger, F. Tuczek, Chem. Eur. J. 2000, 6, 519-522.

[79] S. Itoh, H. Kumei, M. Taki, S. Nagatomo, T. Kitagawa, S. Fukuzumi, J. Am. Chem. Soc. 2001, 123, 6708-6709.

[80] A. Hoffmann, C. Citek, S. Binder, A. Goos, M. Rübhausen, O. Troeppner, I. Ivanovic-Burmazovic, E. C. Wasinger, T. D. P. Stack, S. Herres-Pawlis, ACIE 2013, 52, 5398-5401.

[81] Z. Huang, O. Kwon, K. V. N. Esguerra, J.-p. Lumb, Tetrahedron 2015, 71, 58715885.

[82] K. D. Karlin, J. C. Hayes, Y. Gultneh, R. W. Cruse, J. W. McKown, J. P. Hutchinson, J. Zubieta, J. Am. Chem. Soc. 1984, 106, 2121-2128.

[83] K. D. Karlin, B. I. Cohen, A. Farooq, S. Liu, J. Zubieta, Inorg. Chim. Acta 1988, 153, 9-11.

[84] M. Rolff, J. N. Hamann, F. Tuczek, ACIE 2011, 50, 6924-6927.

[85] J. N. Hamann, M. Rolff, F. Tuczek, Dalton Trans. 2015, 44, 3251-3258.

[86] L. Casella, E. Monzani, M. Gullotti, D. Cavagnino, G. Cerina, L. Santagostini, R. Ugo, Inorg. Chem. 1996, 35, 7516-7525.

[87] E. Monzani, L. Quinti, A. Perotti, L. Casella, M. Gullotti, L. Randaccio, S. Geremia, G. Nardin, P. Faleschini, G. Tabbì, Inorg. Chem. 1998, 37, 553-562. 
[88] E. Monzani, G. Battaini, A. Perotti, L. Casella, M. Gullotti, L. Santagostini, G. Nardin, L. Randaccio, S. Geremia, P. Zanello, G. Opromolla, Inorg. Chem. 1999, 38, 5359-5369.

[89] M. Réglier, C. Jorand, B. Waegell, J. Chem. Soc. Chem. Commun. 1990, 1752.

[90] M. Rolff, J. Schottenheim, G. Peters, F. Tuczek, ACIE 2010, 49, 6438-6442.

[91] J. Schottenheim, N. Fateeva, W. Thimm, J. Krahmer, F. Tuczek, ZAAC 2013, 639, 1491-1497.

[92] J. N. Hamann, F. Tuczek, Chem. Commun. 2014, 50, 2298-2300.

[93] A. Hoffmann, U. Flörke, M. Schürmann, S. Herres-Pawlis, Eur. J. Org. Chem. 2010, 4136-4144.

[94] A. C. Mendes, E. T. Baran, R. L. Reis, H. S. Azevedo, Wiley Interdiscip. Rev. Nanomedicine Nanobiotechnology 2013, 5, 582-612.

[95] D. Philp, J. F. Stoddart, ACIE 1996, 35, 1154-1196.

[96] J. Meyer, J. Biol. Inorg. Chem. 2008, 13, 157-170.

[97] L. M. Mirica, M. Vance, D. J. Rudd, B. Hedman, K. O. Hodgson, E. I. Solomon, T. D. P. Stack, J. Am. Chem. Soc. 2002, 124, 9332-9333.

[98] L. M. Mirica, D. J. Rudd, M. A. Vance, E. I. Solomon, K. O. Hodgson, B. Hedman, T. D. P. Stack, J. Am. Chem. Soc. 2006, 128, 2654-2665.

[99] L. M. Mirica, M. Vance, D. J. Rudd, B. Hedman, K. O. Hodgson, E. I. Solomon, T. D. P. Stack, Science 2005, 308, 1890-1892.

[100] J. Reedijk, Science 2005, 308, 1876-1877.

[101] B. T. Op, M. A. Vance, L. M. Mirica, D. E. Heppner, T. D. P. Stack, E. I. Solomon, J. Am. Chem. Soc. 2009, 131, 6421-6438.

[102] W. B. Tolman, D. J. E. Spencer, Curr. Opin. Chem. Biol. 2001, 5, 188-195.

[103] C. W. Liu, R. J. Staples, J. P. Fackler, Coord. Chem. Rev. 1998, 174, 147-177.

[104] K. Fujisawa, Y. Moro-oka, N. Kitajima, J. Chem. Soc. Chem. Commun. 1994, 379, 623-624.

[105] D. W. Randall, D. R. Gamelin, L. B. LaCroix, E. I. Solomon, J. Biol. Inorg. Chem. JBIC a Publ. Soc. Biol. Inorg. Chem. 2000, 5, 16-29. 
[106] A. C. Rosenzweig, T. V. O'Halloran, Curr. Opin. Chem. Biol. 2000, 4, 140-147.

[107] T. Rasmussen, B. C. Berks, J. Sanders-Loehr, D. M. Dooley, W. G. Zumft, A. J. Thomson, Biochemistry 2000, 39, 12753-12756.

[108] M. E. Helton, P. Chen, P. P. Paul, R. D. Sommer, L. N. Zakharov, A. L. Rheingold, E. I. Solomon, K. D. Karlin, J. Am. Chem. Soc. 2003, 1160-1161.

[109] P. Chen, K. Fujisawa, M. E. Helton, K. D. Karlin, E. I. Solomon, J. Am. Chem. Soc. 2003, 125, 6394-6408.

[110] S. Zhang, M. M. Melzer, S. N. Sen, N. Çelebi-Ölçüm, T. H. Warren, Nature Chem. 2016, 8, 663-669.

[111] D. Maiti, J. S. Woertink, M. A. Vance, A. E. Milligan, A. A. Narducci Sarjeant, E. I. Solomon, K. D. Karlin, J. Am. Chem. Soc. 2007, 129, 8882-8892.

[112] J. Srogl, J. Hývl, A. Révész, D. Schröder, Chem. Commun. 2009, 3463-3465.

[113] U. P. Chaudhuri, D. R. Powell, R. P. Houser, Inorg. Chim. Acta 2009, 362, 23712378.

[114] M. C. Kozlowski, B. J. Morgan, E. C. Linton, Chem. Soc. Rev. 2009, 38, 3193.

[115] M. Sainsbury, Tetrahedron 1980, 36, 3327-3359.

[116] G. Bringmann, R. Walter, R. Weirich, ACIE 1990, 29, 977-991.

[117] J. Hassan, M. Sévignon, C. Gozzi, E. Schulz, M. Lemaire, Chem. Rev. 2002, 102, $1359-1470$.

[118] F. Ullmann, M. Zlokasoff, Berichte der Dtsch. Chem. Gesellschaft 1905, 38, 21112119.

[119] K. V. N. Esguerra, Y. Fall, J. P. Lumb, ACIE 2014, 53, 5877-5881.

[120] K. V. N. Esguerra, Y. Fall, L. Petitjean, J. P. Lumb, J. Am. Chem. Soc. 2014, 136, $7662-7668$.

[121] M. S. Askari, M. Orio, X. Ottenwaelder, Chem. Commun. 2015, 51, 11206-11209.

[122] K. V. N. Esguerra, Synlett 2015, 26, 2731-2738.

[123] M. S. Askari, K. V. N. Esguerra, J.-p. Lumb, X. Ottenwaelder, Inorg. Chem. 2015, $54,8665-8672$. 
[124] L. Chaloner, A. Khomutovskaya, F. Thomas, X. Ottenwaelder, Dalton Trans. 2016, 45, 11109-11119.

[125] Z. Huang, J. P. Lumb, ACIE 2016, 55, 11543-11547.

[126] K. V. N. Esguerra, Y. Fall, J. P. Lumb, Inorg. Chim. Acta 2017, 3-6.

[127] K. V. N. Esguerra, W. Xu, J. P. Lumb, Chem 2017, 2, 533-549.

[128] O. Kwon, K. V. N. Esguerra, M. Glazerman, L. Petitjean, Y. Xu, X. Ottenwaelder, J. P. Lumb, Synlett 2017, 28, 1548-1553.

[129] K. V. N. Esguerra, J. P. Lumb, Chem. Eur. J. 2017, 23, 8596-8600.

[130] K. V. N. Esguerra, J. P. Lumb, ACS Catal. 2017, 7, 3477-3482.

[131] A. S. Hay, H. S. Blanchard, G. F. Endres, J. W. Eustance, J. Am. Chem. Soc. 1959, 81, 6335-6336.

[132] P. J. Baesjou, W. L. Driessen, G. Challa, J. Reedijk, J. Mol. Catal. A: Chem. 1996, $110,195-210$.

[133] J. K. Fink, Handbook of Engineering and Speciality Thermoplastics Volume 3, Vol. 1, 2011.

[134] L. M. Wan, H. X. Li, W. Zhao, H. Y. Ding, Y. Y. Fang, P. H. Ni, J. P. Lang, J. Polym. Sci. Part A: Polym. Chem. 2012, 50, 4864-4870.

[135] P. Gamez, J. A. P. P. Van Dijk, W. L. Driessen, G. Challa, J. Reedijk, Adv. Synth. Catal. 2002, 344, 890-893.

[136] K. Saito, T. Tago, T. Masuyama, H. Nishide, ACIE 2004, 43, 730-733.

[137] C. W. Chen, I. H. Lin, C. C. Lin, J. L. Lin, M. Horie, Polym. (United Kingdom) 2013, 54, 5684-5690.

[138] K. Saito, S. Pant, M. T. W. Hearn, J. Appl. Polym. Sci. 2011, 122, 2174-2180.

[139] H.-Y. Chen, H.-W. Lee, S.-C. Hua, K.-J. Chen, C.-W. Chen, I.-H. Lin, C.-C. Lin, M. Horie, J. Polym. Res. 2016, 23, 248.

[140] Q. Liu, H. G. Wu, C. R. Chim. 2013, 16, 451-461.

[141] X. Sun, R. Bai, Y. Zhang, Q. Wang, X. Fan, J. Yuan, L. Cui, P. Wang, Appl. Biochem. Biotechnol. 2013, 171, 1673-1680. 
[142] P. J. Baesjou, W. L. Driessen, G. Challa, J. Reedijk, J. Am. Chem. Soc. 1997, 119, 12590-12594.

[143] P. Gamez, S. Gupta, J. Reedijk, C. R. Chim. 2007, 10, 295-304.

[144] A. Suzuki, ACIE 2011, 50, 6722-6737.

[145] J. Wencel-Delord, F. Glorius, Nature Chem. 2013, 5, 369-375.

[146] C. Shimokawa, S. Yokota, Y. Tachi, N. Nishiwaki, M. Ariga, S. Itoh, Inorg. Chem. 2003, 42, 8395-8405.

[147] C. Shimokawa, J. Teraoka, Y. Tachi, S. Itoh, J. Inorg. Biochem. 2006, 100, 1118 1127.

[148] S.-I. Murahashi, Y. Oda, T. Naota, N. Komiya, J. Chem. Soc. Chem. Commun. 1993, 2, 139 - 140.

[149] N. Komiya, T. Naota, S. I. Murahashi, Tetrahedron Lett. 1996, 37, 1633-1636.

[150] M. Costas, A. Llobet, J. Mol. Catal. A: Chem. 1999, 142, 113-124.

[151] M. Shimizu, Y. Watanabe, H. Orita, T. Hayakawa, K. Takehira, Tetrahedron Lett. 1991, 32, 2053-2056.

[152] R. H. H. van den Heuvel, M. W. Fraaije, M. Ferrer, A. Mattevi, W. J. H. van Berkel, Proc. Natl. Acad. Sci. 2000, 97, 9455-9460.

[153] C. Boldron, P. Gamez, D. M. Tooke, A. L. Spek, J. Reedijk, ACIE 2005, 44, $3585-3587$.

[154] C. Boldron, e. Özalp-Yaman, P. Gamez, D. M. Tooke, A. L. Spek, J. Reedijk, Dalton Trans. 2005, 3535 .

[155] A. Prokofieva, A. I. Prikhod'ko, S. Dechert, F. Meyer, Chem. Commun. 2008, $1005-1007$.

[156] A. Prokofieva, PhD thesis, Georg-August-Universität Göttingen, 2008.

[157] P. Milko, J. Roithova, N. Tsierkezos, D. Schröder, J. Am. Chem. Soc. 2008, 130, $7186-7187$.

[158] B. A. Jazdzewski, P. L. Holland, M. Pink, V. G. Young, D. J. E. Spencer, W. B. Tolman, Inorg. Chem. 2001, 40, 6097-6107. 
[159] J. A. Halfen, B. A. Jazdzewski, S. Mahapatra, L. M. Berreau, E. C. Wilkinson, L. Que, W. B. Tolman, J. Am. Chem. Soc. 1997, 119, 8217-8227.

[160] L. Que, Coord. Chem. Rev. 1983, 50, 73-108.

[161] A. Mukherjee, M. L. Mcglashen, T. G. Spiro, J. Phys. Chem. 1995, 70, 4912-4917.

[162] M. McGlashen, D. Eads, J. Phys. Chem. 1995, 4918-4922.

[163] J. Lorösch, W. Haase, P. V. Huong, J. Inorg. Biochem. 1986, 27, 53-63.

[164] A. Sokolowski, H. Leutbecher, T. Weyhermüller, R. Schnepf, E. Bothe, E. Bill, P. Hildebrandt, K. Wieghardt, J. Biol. Inorg. Chem. 1997, 2, 444-453.

[165] R. Schnepf, A. Sokolowski, J. Müller, V. Bachler, K. Wieghardt, P. Hildebrandt, J. Am. Chem. Soc. 1998, 120, 2352-2364.

[166] J. C. Röder, F. Meyer, H. Pritzkow, Organometallics 2001, 20, 811-817.

[167] A. L. Gavrilova, B. Bosnich, Chem. Rev. 2004, 104, 349-383.

[168] J. Klingele, S. Dechert, F. Meyer, Coord. Chem. Rev. 2009, 253, 2698-2741.

[169] C. Würtele, E. Gaoutchenova, K. Harms, M. C. Holthausen, J. Sundermeyer, S. Schindler, ACIE 2006, 45, 3867-3869.

[170] S. Schindler, Eur. J. Inorg. Chem. 2000, 2311-2326.

[171] L. Siegfried, T. A. Kaden, F. Meyer, P. Kircher, H. Pritzkow, J. Chem. Soc. Dalt. Trans. 2001, 2310-2315.

[172] S. Buchler, F. Meyer, E. Kaifer, H. Pritzkow, Inorg. Chim. Acta 2002, 337, 371-386.

[173] J. C. Röder, F. Meyer, E. Kaifer, H. Pritzkow, Eur. J. Inorg. Chem. 2004, 16461660.

[174] J. Ackermann, F. Meyer, H. Pritzkow, Inorg. Chim. Acta 2004, 357, 3703-3711.

[175] G. Noël, J. C. Röder, S. Dechert, H. Pritzkow, L. Bolk, S. Mecking, F. Meyer, Adv. Synth. Catal. 2006, 348, 887-897.

[176] L. Casella, E. Monzani, B. M. Gullotti, F. Gliubichb, L. D. Gioiab, Dalton Trans. 1994, 3203-3210.

[177] J. S. Bradshaw, R. B. Nielsen, P.-k. Tse, G. Arena, B. E. Wilson, N. K. Dalley, J. D. Lamb, J. J. Christensen, R. M. Izatt, J. Heterocycl. Chem. 1986, 23, 361-268. 
[178] T. G. Schenck, J. M. Downes, C. R. C. Milne, P. B. Mackenzie, H. Boucher, J. Whelan, B. Bosnich, Inorg. Chem. 1992, 24, 2334-2337.

[179] K. J. Oberhausen, J. F. Richardson, R. M. Buchanan, W. Pierce, Polyhedron 1989, 8, 659-668.

[180] M.-c. Rodriguez, J. Guilhem, B. Keita, L. Nadjo, R. V. August, Inorg. Chem. 1996, 35, 7804-7810.

[181] K. Itoh, H. Hayashi, H. Furutachi, T. Matsumoto, S. Nagatomo, T. Tosha, S. Terada, S. Fujinami, M. Suzuki, T. Kitagawa, J. Am. Chem. Soc. 2005, 127, 5212-5223.

[182] Z. Li, A. Zhu, J. Yang, J. Heterocycl. Chem. 2012, 49, 1458 -1461.

[183] D. G. Blackmond, ACIE 2005, 44, 4302-4320.

[184] S. C. Hyung, W. K. Jun, Y. N. Cha, C. Kim, J. Immunoass. Immunochem. 2006, 27, 31-44.

[185] B. Porstmann, T. Porstmann, E. Nugel, Clin. Chem. Lab. Med. 1981, 19, 435-439.

[186] A. Fallarini, C. Redaelli, E. Monzani, B. Bonafe, Biochim. Biophys. Acta-Bioenerg. 2001, 1547, 302-312.

[187] M. K. Nazeeruddin, S. M. Zakeeruddin, K. Kalyanasundaram, J. Phys. Chem. 1993, 97, 9607-9612.

[188] J. W. Pyrz, K. D. Karlin, T. N. Sorrell, G. C. Vogel, L. Que, Inorg. Chem. 1984, 23, 4581-4584.

[189] S. Ghosh, J. Cirera, M. A. Vance, T. Ono, K. Fujisawa, E. I. Solomon, J. Am. Chem. Soc. 2008, 130, 16262-16273.

[190] W. Y. Shiu, K. C. Ma, D. Varhanícková, D. Mackay, Cancer Lett. 1990, 49, 11551224.

[191] K. Kaupmees, A. Trummal, I. Leito, Croat. Chem. Acta 2014, 87, 385-395.

[192] I. Kaljurand, A. Kütt, L. Sooväli, T. Rodima, V. Mäemets, I. Leito, I. A. Koppel, J. Org. Chem. 2005, 70, 1019-1028.

[193] M. Chanda, R. Salil, Industrial Polymers, Specialty Polymers, and Their Applications (Plastics Engineering), CRC Press Inc., 2008.

[194] C. L. Perrin, Y. Dong, J. Am. Chem. Soc. 2007, 129, 4490-4497. 
[195] J. L. Bolton, H. M. Wu, L. Q. Hu, Chem. Res. Toxicol. 1996, 9, 109-113.

[196] A. Streitwieser, R. H. Jagow, R. C. Fahey, S. Suzuki, J. Am. Chem. Soc. 1958, 80, $2326-2332$.

[197] I. Lee, Chem. Soc. Rev. 1995, 24, 223.

[198] H. Amouri, Synlett 2011, 1357-1369.

[199] H. Amouri, J. Le Bras, Acc. Chem. Res. 2002, 35, 501-510.

[200] Q. Zhou, K. D. Turnbull, J. Org. Chem. 2001, 66, 7072-7.

[201] X. Lei, C.-H. Jiang, X. Wen, Q.-L. Xu, H. Sun, RSC Adv. 2015, 5, 14953-14957.

[202] C. Jarava-Barrera, A. Parra, A. López, F. Cruz-Acosta, D. Collado-Sanz, D. J. Cárdenas, M. Tortosa, ACS Catal. 2016, 6, 442-446.

[203] W. Baik, H. J. Lee, C. H. Yoo, J. W. Jung, B. H. Kim, J. Chem. Soc. Perkin Trans. 1 1997, 587-590.

[204] X. Z. Zhang, J. Y. Du, Y. H. Deng, W. D. Chu, X. Yan, K. Y. Yu, C. A. Fan, J. Org. Chem. 2016, 81, 2598-2606.

[205] X. Li, X. Xu, W. Wei, A. Lin, H. Yao, Org. Lett. 2016, 18, 428-431.

[206] Y. Shen, J. Qi, Z. Mao, S. Cui, Org. Lett. 2016, 18, 2722-2725.

[207] Z. Yuan, W. Wei, A. Lin, H. Yao, Org. Lett. 2016, 18, 3370-3373.

[208] K. Zhao, Y. Zhi, A. Wang, D. Enders, ACS Catal. 2016, 6, 657-660.

[209] M. Kretzschmar, T. Hodk, C. Schneider, ACIE 2016, 55, 9788-9792.

[210] A. Vigalok, D. Milstein, Acc. Chem. Res. 2001, 34, 798-807.

[211] A. D. MacIntosh, H. Yang, R. D. Pike, D. A. Sweigart, J. Organomet. Chem. 2012, $719,14-17$.

[212] H. Amouri, Y. Besace, J. L. Bras, J. Vaissermann, J. Am. Chem. Soc. 1998, 120, $6171-6172$.

[213] A. Vigalok, D. Milstein, J. Am. Chem. Soc. 1997, 119, 7873-7874.

[214] M. A. Todd, M. L. Grachan, M. Sabat, W. H. Myers, W. D. Harman, Organometallics 2006, 25, 3948-3954.

[215] O. Rabin, V. Arkadi, D. Milstein, Chem. Eur. J. 2000, 6, 454-462. 
[216] M. Benson, L. Jurd, Org. Magn. Reson. 1984, 22, 86-89.

[217] K.-T. Wei, I. Paul, G. Le Coustumer, R. Pinel, Y. Mollier, Tetrahedron Lett. 1977, 18, 2717-2720.

[218] L. K. Dyall, S. Winstein, J. Am. Chem. Soc. 1972, 94, 2196-2199.

[219] L. J. Filar, S. Winstein, Tetrahedron Lett. 1960, 1, 9-16.

[220] M. S. Singh, A. Nagaraju, N. Anand, S. Chowdhury, RSC Adv. 2014, 4, 5592455959.

[221] Z. Wang, J. Sun, Org. Lett. 2017, 19, 2334-2337.

[222] Y. Xie, B. List, ACIE 2017, 56, 4936-4940.

[223] C. Ma, Y. Huang, Y. Zhao, ACS Catal. 2016, 6, 6408-6412.

[224] B. T. Ramanjaneyulu, S. Mahesh, R. V. Anand, Org. Lett. 2015, 17, 3952-3955.

[225] A. Parra, M. Tortosa, ChemCatChem 2015, 7, 1524-1526.

[226] J.-A. Jiang, C. Chen, J.-G. Huang, H.-W. Liu, S. Cao, Y.-F. Ji, Green Chem. 2014, $16,1248-1254$.

[227] W. Baik, H. J. Lee, J. M. Jang, S. Koo, B. H. Kim, J. Org. Chem. 2000, 65, $108-115$.

[228] X. Sun, Z. M. a. Judeh, B. F. Ali, S. F. Alshahateet, Catal. Today 2008, 131, $423-426$.

[229] B. Barton, C. G. Logie, B. M. Schoonees, B. Zeelie, Org. Process Res. Dev. 2005, 8 , year.

[230] N. de Heij, R. Sheldon in The role of oxygen in chemistry and biochemistry, Elsevier, Amsterdam, 1988, pp. 243-256.

[231] A. M. Wright, G. Wu, T. W. Hayton, J. Am. Chem. Soc. 2010, 132, 14336-14337.

[232] A. Kalita, P. Kumar, C. Deka, B. Mondal, Chem. Commun. 2012, 48, 1251-1253.

[233] P. Kumar, A. Kalita, B. Mondal, Inorg. Chim. Acta 2013, 404, 88-96.

[234] V. A. Online, L. Wang, G. Wang, H. Qu, Z. H. Li, M. Zhou, Phys. Chem. Chem. Phys. 2014, 16, 10788-10798.

[235] A. Kalita, V. Kumar, B. Mondal, Inorg. Chim. Acta 2015. 
[236] T. Ghosh, B. Mondal, T. Ghosh, M. Sutradhar, G. Mukherjee, M. G. B. Drew, Inorg. Chim. Acta 2007, 360, 1753-1761.

[237] T. Ghosh, B. Mondal, R. Patra, Transit. Met. Chem. 2007, 32, 468-474.

[238] S. Zhang, C. Nihan, M. M. Melzer, K. N. Houk, T. H. Warren, J. Am. Chem. Soc. 2013, 135, 16746-16749.

[239] K. Nakamoto in Handbook of Vibrational Spectroscopy 6th Ed. (Ed.: P. R. Griffiths), John Wiley \& Sons, Ltd, Chichester, UK, 6th ed., 2006.

[240] C. E. Ruggiero, S. M. Carrier, W. E. Antholine, J. W. Whittaker, C. J. Cramer, W. B. Tolman, J. Am. Chem. Soc. 1993, 115, 11285-11298.

[241] G. B. Wijeratne, S. Hematian, M. A. Siegler, K. D. Karlin, J. Am. Chem. Soc. 2017, jacs.7b07808.

[242] M. E. Chacón Villalba, C. A. Franca, J. A. Güida, Spectrochim. Acta - Part A Mol. Biomol. Spectrosc. 2017, 176, 189-196.

[243] L. Kuhn, E. R. Lippincott, J. Am. Chem. Soc. 1956, 78, 1820-1821.

[244] C. Varotsis, T. Ohta, T. Kitagawa, T. Soulimane, E. Pinakoulaki, ACIE 2007, 46, 2210-2214.

[245] M. E. Villalba, A. Navaza, J. A. Güida, E. L. Varetti, P. J. Aymonino, Inorg. Chim. Acta 2006, 359, 707-712.

[246] G. J. Kubas, B. Monzyk, A. L. Crumbliss in Inorg. Synth., 2007, pp. 90-92.

[247] S. Stoll, A. Schweiger, J. Magn. Reson. 2006, 178, 42-55.

[248] L. Casella, M. Gullotti, R. Radaelli, P. Di Gennaro, J. Chem. Soc. Chem. Commun. 1991, 1611.

[249] A. Thurkauf, A. Hutchison, J. Peterson, L. Cornfield, R. Meade, K. Huston, K. Harris, P. C. Ross, K. Gerber, T. V. Ramabhadrano, J. Med. Chem 1995, 2251-2255.

[250] A. Prokofieva, A. I. Prikhod'ko, E. A. Enyedy, E. Farkas, W. Maringgele, S. Demeshko, S. Dechert, F. Meyer, Inorg. Chem. 2007, 46, 4298-4307. 


\section{Chrystallographic Data}

Table 8.1 Crystal data and refinement details for $\mathbf{2 ,} \mathbf{3}$, and $\mathbf{4}$.

\begin{tabular}{|c|c|c|c|}
\hline compound & 2 & 3 & 4 \\
\hline empirical formula & $\mathrm{C}_{62} \mathrm{H}_{84} \mathrm{Cl}_{4} \mathrm{Cu}_{4} \mathrm{~N}_{30} \mathrm{O}_{16}$ & $\mathrm{C}_{38} \mathrm{H}_{52} \mathrm{Cl}_{2} \mathrm{Cu}_{2} \mathrm{~N}_{12} \mathrm{O}_{12}$ & $\mathrm{C}_{45} \mathrm{H}_{49} \mathrm{Cu}_{2} \mathrm{~N}_{15} \mathrm{O}_{10}$ \\
\hline formula weight & 1901.55 & 1066.89 & 1087.07 \\
\hline$T[\mathrm{~K}]$ & $133(2)$ & $133(2)$ & $133(2)$ \\
\hline crystal size $\left[\mathrm{mm}^{3}\right]$ & $0.330 \times 0.140 \times 0.070$ & $0.210 \times 0.170 \times 0.100$ & $0.410 \times 0.220 \times 0.140$ \\
\hline crystal system & monoclinic & triclinic & triclinic \\
\hline space group & $P 2_{1} / c($ No. 14$)$ & $P \overline{1}($ No. 2$)$ & $P \overline{1}($ No. 2$)$ \\
\hline$a[\AA]$ & $13.7463(5)$ & $12.1710(5)$ & $12.1627(5)$ \\
\hline$b[\AA]$ & $24.5829(6)$ & $12.4574(5)$ & $13.7045(6)$ \\
\hline$c[\AA]$ & $11.9537(4)$ & $17.1454(6)$ & $14.3981(6)$ \\
\hline$\alpha\left[^{\circ}\right]$ & 90 & $103.484(3)$ & $98.711(4)$ \\
\hline$\beta\left[^{\circ}\right]$ & $98.316(3)$ & $100.319(3)$ & $102.756(3)$ \\
\hline$\gamma\left[^{\circ}\right]$ & 90 & $111.250(3)$ & $95.319(4)$ \\
\hline$V\left[\AA^{3}\right]$ & $3997.0(2)$ & $2254.15(16)$ & $2293.81(17)$ \\
\hline$Z$ & 2 & 2 & 2 \\
\hline$\rho\left[\mathrm{g} \cdot \mathrm{cm}^{-3}\right]$ & 1.580 & 1.572 & 1.574 \\
\hline$F(000)$ & 1956 & 1104 & 1124 \\
\hline$\mu\left[\mathrm{mm}^{-1}\right]$ & 1.266 & 1.137 & 1.005 \\
\hline$T_{\min } / T_{\max }$ & $0.7546 / 0.9171$ & $0.7586 / 0.9154$ & $0.6445 / 0.8279$ \\
\hline$\theta$-range $\left[^{\circ}\right]$ & $1.657-25.624$ & $1.876-26.718$ & $1.473-26.096$ \\
\hline \multirow[t]{3}{*}{$h k l$-range } & $-16 \leq h \leq 14$ & $-15 \leq h \leq 15$ & $-15 \leq h \leq 15$ \\
\hline & $-29 \leq k \leq 29$ & $-15 \leq k \leq 15$ & $-16 \leq k \leq 16$ \\
\hline & $-14 \leq l \leq 29$ & $-21 \leq l \leq 21$ & $-17 \leq l \leq 17$ \\
\hline measured refl. & 42334 & 25158 & 43002 \\
\hline unique refl. $\left[R_{\mathrm{int}}\right]$ & $7531[0.0732]$ & 9558 [0.0423] & $43002[?]$ \\
\hline obs. refl. $(I>2 \sigma(I))$ & 6166 & 7429 & 32945 \\
\hline data / restr. / param. & $7531 / 0 / 530$ & $9558 / 27 / 712$ & $43002 / 25 / 697$ \\
\hline goodness-of-fit $\left(F^{2}\right)$ & 1.092 & 0.965 & 0.958 \\
\hline$R 1, w R 2(I>2 \sigma(I))$ & $0.0442 / 0.0938$ & $0.0352 / 0.0763$ & $0.0539 / 0.1279$ \\
\hline$R 1, w R 2$ (all data) & $0.0602 / 0.0991$ & $0.0534 / 0.0810$ & $0.0703 / 0.1345$ \\
\hline res. el. dens. $\left[\mathrm{e} \cdot \AA^{-3}\right]$ & $-0.322 / 0.659$ & $-0.493 / 0.818$ & $-0.521 / 0.890$ \\
\hline
\end{tabular}


Table 8.2 Crystal data and refinement details for $\mathbf{5}$ and $\mathbf{7}$.

\begin{tabular}{|c|c|c|}
\hline compound & 5 & 7 \\
\hline empirical formula & $\mathrm{C}_{47} \mathrm{H}_{54} \mathrm{Cu}_{2} \mathrm{~N}_{15} \mathrm{O}_{8}$ & $\mathrm{C}_{53.50} \mathrm{H}_{55.75} \mathrm{Cl}_{2.25} \mathrm{Cu}_{2} \mathrm{~N}_{12} \mathrm{O}_{10.25}$ \\
\hline formula weight & 1084.13 & 1237.69 \\
\hline$T[\mathrm{~K}]$ & $133(2)$ & $133(2)$ \\
\hline crystal size $\left[\mathrm{mm}^{3}\right]$ & $0.500 \times 0.290 \times 0.050$ & $0.500 \times 0.320 \times 0.230$ \\
\hline crystal system & triclinic & triclinic \\
\hline space group & $P \overline{1}($ No. 2$)$ & $P \overline{1}($ No. 2$)$ \\
\hline$a[\AA]$ & $9.5434(3)$ & $11.7615(7)$ \\
\hline$b[\AA]$ & $16.0564(6)$ & $12.1187(5))$ \\
\hline$c[\AA]$ & $16.1091(6)$ & $23.6549(14))$ \\
\hline$\alpha\left[^{\circ}\right]$ & $99.724(3)$ & $92.570(4$ \\
\hline$\beta\left[^{\circ}\right]$ & $98.018(3)$ & $92.412(5)$ \\
\hline$\gamma\left[^{\circ}\right]$ & $97.799(3)$ & $117.201(4)$ \\
\hline$V\left[\AA^{3}\right]$ & $2376.90(15)$ & $2988.3(3)$ \\
\hline$Z$ & 2 & 2 \\
\hline$\rho\left[\mathrm{g} \cdot \mathrm{cm}^{-3}\right]$ & 1.515 & 1.376 \\
\hline$F(000)$ & 1126 & 1278 \\
\hline$\mu\left[\mathrm{mm}^{-1}\right]$ & 0.967 & 0.877 \\
\hline$T_{\min } / T_{\max }$ & $0.6430 / 0.9315$ & $0.7478 / 0.9087$ \\
\hline$\theta$-range $\left[^{\circ}\right]$ & $1.304-26.748$ & $1.894-26.066$ \\
\hline \multirow[t]{3}{*}{$h k l$-range } & $-11 \leq h \leq 12$ & $-14 \leq h \leq 14$ \\
\hline & $-20 \leq k \leq 20$ & $-14 \leq k \leq 14$ \\
\hline & $-20 \leq l \leq 20$ & $-29 \leq l \leq 29$ \\
\hline measured refl. & 33301 & 42507 \\
\hline unique refl. $\left[R_{\text {int }}\right]$ & $10067[0.0554]$ & $42507[?]$ \\
\hline obs. refl. $(I>2 \sigma(I))$ & 7838 & 30405 \\
\hline data / restr. / param. & $10067 / 55 / 798$ & $42507 / 55 / 7905$ \\
\hline goodness-of-fit $\left(F^{2}\right)$ & 1.039 & 1.080 \\
\hline$R 1, w R 2(I>2 \sigma(I))$ & $0.0482 / 0.1274$ & $0.0716 / 0.2058$ \\
\hline$R 1, w R 2($ all data $)$ & $0.0664 / 0.1356$ & $0.1066 / 0.2390$ \\
\hline res. el. dens. $\left[\mathrm{e} \cdot \AA^{-3}\right]$ & $-0.723 / 0.896$ & $-0.592 / 1.175$ \\
\hline
\end{tabular}

Table 8.3 Selected bond lengths $[\AA]$ for $\mathbf{2}$.

\begin{tabular}{lr}
\hline Atoms & Bond lengths \\
\hline Cu1-N1 & $1.952(2)$ \\
Cu1-N2' & $2.031(2)$ \\
Cu1-N11' & $2.076(2)$ \\
Cu1-N9' & $2.107(2)$ \\
Cu1-N8 & $2.110(2)$ \\
Cu2-N13 & $1.937(3)$ \\
Cu2-N4 & $1.948(3)$ \\
Cu2-N6 & $2.000(3)$ \\
\hline
\end{tabular}


Table 8.4 Selected bond angles $\left[^{\circ}\right]$ for 2.

\begin{tabular}{lc}
\hline Atoms & Bond angles \\
\hline $\mathrm{N} 1-\mathrm{Cu} 1-\mathrm{N} 2^{\prime}$ & $100.55(10)$ \\
$\mathrm{N} 1-\mathrm{Cu} 1-\mathrm{N} 11^{\prime}$ & $96.26(9)$ \\
$\mathrm{N}^{\prime}-\mathrm{Cu} 1-\mathrm{N} 11^{\prime}$ & $117.14(10)$ \\
$\mathrm{N} 1-\mathrm{Cu} 1-\mathrm{N} 9^{\prime}$ & $105.28(10)$ \\
$\mathrm{N} 2^{\prime}-\mathrm{Cu} 1-\mathrm{N} 9^{\prime}$ & $115.29(9)$ \\
$\mathrm{N} 11^{\prime}-\mathrm{Cu} 1-\mathrm{N} 9^{\prime}$ & $117.49(9)$ \\
$\mathrm{N} 1-\mathrm{Cu} 1-\mathrm{N} 8^{\prime}$ & $175.18(10)$ \\
$\mathrm{N} 2^{\prime}-\mathrm{Cu} 1-\mathrm{N} 8^{\prime}$ & $79.64(9)$ \\
$\mathrm{N} 11^{\prime}-\mathrm{Cu} 1-\mathrm{N} 8^{\prime}$ & $79.47(9)$ \\
$\mathrm{N} 9^{\prime}-\mathrm{Cu} 1-\mathrm{N} 8^{\prime}$ & $78.83(9)$ \\
$\mathrm{N} 13-\mathrm{Cu} 2-\mathrm{N} 4$ & $137.47(11)$ \\
$\mathrm{N} 13-\mathrm{Cu} 2-\mathrm{N} 6$ & $104.04(11)$ \\
$\mathrm{N} 4-\mathrm{Cu} 2-\mathrm{N} 6$ & $116.60(11)$ \\
$\mathrm{C} 2-\mathrm{N} 1-\mathrm{Cu} 1$ & $129.50(19)$ \\
$\mathrm{N} 2-\mathrm{N} 1-\mathrm{Cu} 1$ & $119.96(17)$ \\
$\mathrm{C} 3-\mathrm{N} 2-\mathrm{Cu} 1^{\prime}$ & $114.76(19)$ \\
$\mathrm{N} 1-\mathrm{N} 2-\mathrm{Cu} 1^{\prime}$ & $137.34(18)$ \\
$\mathrm{C} 6-\mathrm{N} 4-\mathrm{Cu} 2$ & $125.0(2)$ \\
$\mathrm{C} 7-\mathrm{N} 4-\mathrm{Cu} 2$ & $129.0(2)$ \\
$\mathrm{C} 11-\mathrm{N} 6-\mathrm{Cu} 2$ & $126.5(2)$ \\
$\mathrm{C} 12-\mathrm{N} 6-\mathrm{Cu} 2$ & $127.1(2)$ \\
$\mathrm{C} 21-\mathrm{N} 8-\mathrm{Cu} 1^{\prime}$ & $107.87(17)$ \\
$\mathrm{C} 15-\mathrm{N} 8-\mathrm{Cu} 1^{\prime}$ & $106.44(17)$ \\
$\mathrm{C} 16-\mathrm{N} 8-\mathrm{Cu} 1^{\prime}$ & $107.67(17)$ \\
$\mathrm{C} 17-\mathrm{N} 9-\mathrm{Cu} 1^{\prime}$ & $110.37(19)$ \\
$\mathrm{C} 18-\mathrm{N} 9-\mathrm{Cu} 1^{\prime}$ & $143.6(2)$ \\
$\mathrm{C} 22-\mathrm{N} 11-\mathrm{Cu} 1^{\prime}$ & $110.19(18)$ \\
$\mathrm{C} 23-\mathrm{N} 11-\mathrm{Cu} 1^{\prime}$ & $142.1(2)$ \\
$\mathrm{C} 31-\mathrm{N} 13-\mathrm{Cu} 2$ & $161.6(3)$ \\
\hline
\end{tabular}

Table 8.5 Selected bond lengths $[\AA]$ for $\mathbf{3}$.

\begin{tabular}{lc}
\hline Atoms & Bond lengths \\
\hline Cu1-O4 & $1.9563(16)$ \\
Cu1-N6 & $1.9965(18)$ \\
Cu1-N2 & $2.0010(18)$ \\
Cu1-N3 & $2.0903(18)$ \\
Cu1-N5 & $2.130(2)$ \\
Cu2-O31A & $1.91(3)$ \\
Cu2-O31B & $1.96(11)$ \\
Cu2-N1 & $2.0131(18)$ \\
Cu2-N9 & $2.0207(18)$ \\
Cu2-N8 & $2.1273(18)$ \\
Cu2-N11 & $2.146(2)$ \\
\hline
\end{tabular}


Table 8.6 Selected bond angles $\left[^{\circ}\right]$ for 3 .

\begin{tabular}{lc}
\hline Atoms & Bond angles \\
\hline O4-Cu1-N6 & $97.33(7)$ \\
O4-Cu1-N2 & $96.98(7)$ \\
N6-Cu1-N2 & $134.29(8)$ \\
O4-Cu1-N3 & $176.91(7)$ \\
N6-Cu1-N3 & $82.39(7)$ \\
N2-Cu1-N3 & $81.12(7)$ \\
O4-Cu1-N5 & $102.00(7)$ \\
N6-Cu1-N5 & $110.61(7)$ \\
N2-Cu1-N5 & $108.40(7)$ \\
N3-Cu1-N5 & $80.94(7)$ \\
O31A-Cu2-N1 & $99.6(8)$ \\
O31B-Cu2-N1 & $100(3)$ \\
O31A-Cu2-N9 & $100.8(8)$ \\
O31B-Cu2-N9 & $102(3)$ \\
N1-Cu2-N9 & $133.05(8)$ \\
O31A-Cu2-N8 & $176.9(5)$ \\
O31B-Cu2-N8 & $174.7(15)$ \\
N1-Cu2-N8 & $79.83(7)$ \\
N9-Cu2-N8 & $81.75(7)$ \\
O31A-Cu2-N11 & $98.3(3)$ \\
N1-Cu2-N11 & $111.51(8)$ \\
N9-Cu2-N11 & $106.76(8)$ \\
N8-Cu2-N11 & $79.13(8)$ \\
C1-N1-Cu2 & $114.46(13)$ \\
N2-N1-Cu2 & $137.92(14)$ \\
C3-N2-Cu1 & $113.12(13)$ \\
N1-N2-Cu1 & $138.56(14)$ \\
C10-N3-Cu1 & $109.24(13)$ \\
C4-N3-Cu1 & $104.43(12)$ \\
C5-N3-Cu1 & $110.08(13)$ \\
C14-N5-Cu1 & $108.83(15)$ \\
C11-N5-Cu1 & $144.04(16)$ \\
C6-N6-Cu1 & $113.62(15)$ \\
C7-N6-Cu1 & $139.59(15)$ \\
\hline & \\
&
\end{tabular}


Table 8.7 Selected bond lengths $[\AA]$ for $\mathbf{5}$.

\begin{tabular}{lr}
\hline Atoms & Bond lengths \\
\hline $\mathrm{Cu} 1-\mathrm{N} 1$ & $1.957(3)$ \\
$\mathrm{Cu} 1-\mathrm{N} 6$ & $1.989(3)$ \\
$\mathrm{Cu} 1-\mathrm{O} 1$ & $2.074(3)$ \\
$\mathrm{Cu} 1-\mathrm{N} 4$ & $2.110(4)$ \\
$\mathrm{Cu} 1-\mathrm{N} 3$ & $2.198(3)$ \\
$\mathrm{Cu} 2-\mathrm{N} 2$ & $1.966(3)$ \\
$\mathrm{Cu} 2-\mathrm{N} 11$ & $1.997(3)$ \\
$\mathrm{Cu} 2-\mathrm{O} 4$ & $2.038(3)$ \\
$\mathrm{Cu} 2-\mathrm{N} 9$ & $2.073(4)$ \\
$\mathrm{Cu} 2-\mathrm{N} 8$ & $2.226(3)$ \\
\hline
\end{tabular}


Table 8.8 Selected bond angles [ $\left.{ }^{\circ}\right]$ for $\mathbf{5}$.

\begin{tabular}{lr}
\hline Atoms & Bond angles \\
\hline N1-Cu1-N6 & $169.59(14)$ \\
N1-Cu1-O1 & $90.36(13)$ \\
N6-Cu1-O1 & $88.57(13)$ \\
N1-Cu1-N4 & $92.57(14)$ \\
N6-Cu1-N4 & $95.66(14)$ \\
O1-Cu1-N4 & $133.63(12)$ \\
N1-Cu1-N3 & $80.27(13)$ \\
N6-Cu1-N3 & $92.78(13)$ \\
$\mathrm{O} 1-\mathrm{Cu} 1-\mathrm{N} 3$ & $131.91(12)$ \\
N4-Cu1-N3 & $94.09(13)$ \\
N2-Cu2-N11 & $169.36(14)$ \\
N2-Cu2-O4 & $90.52(13)$ \\
N11-Cu2-O4 & $90.10(13)$ \\
N2-Cu2-N9 & $94.45(14)$ \\
N11-Cu2-N9 & $92.97(14)$ \\
O4-Cu2-N9 & $132.32(12)$ \\
N2-Cu2-N8 & $79.53(13)$ \\
N11-Cu2-N8 & $92.32(13)$ \\
O4-Cu2-N8 & $132.98(12)$ \\
N9-Cu2-N8 & $94.44(13)$ \\
C2-N1-Cu1 & $116.2(3)$ \\
N2-N1-Cu1 & $135.1(3)$ \\
C3-N2-Cu2 & $115.0(3)$ \\
N1-N2-Cu2 & $136.2(3)$ \\
C5-N3-Cu1 & $112.7(2)$ \\
C15-N3-Cu1 & $111.1(3)$ \\
C4-N3-Cu1 & $104.1(2)$ \\
C7-N4-Cu1 & $120.4(3)$ \\
C8-N4-Cu1 & $132.4(3)$ \\
C17-N6-Cu1 & $126.3(3)$ \\
C18-N6-Cu1 & $125.9(3)$ \\
C36-N8-Cu2 & $111.3(2)$ \\
C26-N8-Cu2 & $115.0(2)$ \\
C25-N8-Cu2 & $101.2(2)$ \\
C28-N9-Cu2 & $121.1(3)$ \\
C29-N9-Cu2 & $133.1(3)$ \\
C38-N11-Cu2 & $125.4(3)$ \\
$\mathrm{C} 39-\mathrm{N} 11-\mathrm{Cu2}$ & $126.0(3)$ \\
N13-O1-Cu1 & $110.7(2)$ \\
N14-O4-Cu2 & $111.9(2)$ \\
\hline
\end{tabular}


Table 8.9 Selected bond lengths $[\AA]$ for 7 .

\begin{tabular}{lc}
\hline Atoms & Bond lengths \\
\hline Cu1-N1 & $1.976(2)$ \\
Cu1-N6 & $1.994(2)$ \\
Cu1-O1 & $2.021(2)$ \\
Cu1-N3 & $2.107(3)$ \\
Cu1-N4 & $2.309(3)$ \\
O2-Cu2B & $1.848(7)$ \\
O2-Cu2A & $1.880(4)$ \\
N2-Cu2B & $1.988(7)$ \\
N2-Cu2A & $2.030(4)$ \\
N8-Cu2A & $2.036(5)$ \\
N8-Cu2B & $2.077(7)$ \\
N9-Cu2A & $2.100(4)$ \\
N9-Cu2B & $2.278(9)$ \\
Cu2A-N11A & $2.100(7)$ \\
Cu2B-N11B & $2.094(11)$ \\
\hline
\end{tabular}


Table 8.10 Selected bond angles [ $\left.{ }^{\circ}\right]$ for 7 .

\begin{tabular}{lc}
\hline Atoms & Bond angles \\
\hline N1-Cu1-N6 & $158.02(10)$ \\
N1-Cu1-O1 & $90.19(9)$ \\
N6-Cu1-O1 & $91.62(10)$ \\
N1-Cu1-N3 & $80.91(9)$ \\
N6-Cu1-N3 & $93.29(10)$ \\
O1-Cu1-N3 & $167.27(10)$ \\
N1-Cu1-N4 & $105.27(10)$ \\
N6-Cu1-N4 & $96.02(10)$ \\
O1-Cu1-N4 & $99.55(10)$ \\
N3-Cu1-N4 & $91.61(10)$ \\
C2-N1-Cu1 & $114.53(17)$ \\
N2-N1-Cu1 & $136.90(18)$ \\
C3-N2-Cu2B & $114.8(3)$ \\
N1-N2-Cu2B & $137.2(3)$ \\
C3-N2-Cu2A & $112.4(2)$ \\
N1-N2-Cu2A & $140.1(2)$ \\
C15-N3-Cu1 & $117.81(18)$ \\
C4-N3-Cu1 & $103.18(17)$ \\
C5-N3-Cu1 & $110.37(19)$ \\
C7-N4-Cu1 & $117.4(2)$ \\
C8-N4-Cu1 & $128.3(2)$ \\
C17-N6-Cu1 & $123.55(19)$ \\
C23-N6-Cu1 & $129.85(19)$ \\
C26-N8-Cu2A & $114.7(2)$ \\
C25-N8-Cu2A & $106.0(2)$ \\
C36A-N8-Cu2A & $110.3(5)$ \\
C26-N8-Cu2B & $118.2(3)$ \\
C25-N8-Cu2B & $107.1(3)$ \\
C36B-N8-Cu2B & $98.2(10)$ \\
C28-N9-Cu2A & $122.0(3)$ \\
C29-N9-Cu2A & $127.1(3)$ \\
C28-N9-Cu2B & $123.3(3)$ \\
C29-N9-Cu2B & $126.5(3)$ \\
O2-Cu2A-N2 & $91.17(16)$ \\
O2-Cu2A-N8 & $172.8(2)$ \\
N2-Cu2A-N8 & $81.76(17)$ \\
O2-Cu2A-N11A & $96.0(3)$ \\
N2-Cu2A-N11A & $127.0(4)$ \\
N8-Cu2A-N11A & $87.5(3)$ \\
O2-Cu2A-N9 & $93.21(17)$ \\
N2-Cu2A-N9 & $126.00(18)$ \\
N8-Cu2A-N9 & $91.83(16)$ \\
\hline
\end{tabular}


Table 8.11 Selected bond lengths $[\AA]$ for 7 .

\begin{tabular}{lc}
\hline Atoms & Bond lengths \\
\hline $\mathrm{Cu} 1-\mathrm{O} 1$ & $1.925(4)$ \\
$\mathrm{Cu} 1-\mathrm{N} 1$ & $1.972(6)$ \\
$\mathrm{Cu} 1-\mathrm{N} 6$ & $2.028(6)$ \\
$\mathrm{Cu} 1-\mathrm{N} 3$ & $2.117(5)$ \\
$\mathrm{Cu} 1-\mathrm{N} 4$ & $2.150(6)$ \\
$\mathrm{Cu} 2-\mathrm{O} 2$ & $1.912(5)$ \\
$\mathrm{Cu} 2-\mathrm{N} 2$ & $1.974(6)$ \\
$\mathrm{Cu} 2-\mathrm{N} 9$ & $2.046(7)$ \\
$\mathrm{Cu} 2-\mathrm{N} 8$ & $2.109(6)$ \\
$\mathrm{Cu} 2-\mathrm{N} 11$ & $2.120(7)$ \\
\hline
\end{tabular}

Table 8.12 Selected bond angles [ $\left.{ }^{\circ}\right]$ for $\mathbf{7}$.

\begin{tabular}{lr}
\hline Atoms & Bond angles \\
\hline $\mathrm{O} 1-\mathrm{Cu} 1-\mathrm{N} 1$ & $103.0(2)$ \\
$\mathrm{O} 1-\mathrm{Cu} 1-\mathrm{N} 6$ & $92.4(2)$ \\
N1-Cu1-N6 & $135.9(2)$ \\
$\mathrm{O} 1-\mathrm{Cu} 1-\mathrm{N} 3$ & $171.7(2)$ \\
N1-Cu1-N3 & $82.0(2)$ \\
N6-Cu1-N3 & $79.5(2)$ \\
$\mathrm{O} 1-\mathrm{Cu} 1-\mathrm{N} 4$ & $104.7(2)$ \\
N1-Cu1-N4 & $109.5(2)$ \\
N6-Cu1-N4 & $105.8(2)$ \\
N3-Cu1-N4 & $79.4(2)$ \\
$\mathrm{O} 2-\mathrm{Cu} 2-\mathrm{N} 2$ & $103.7(2)$ \\
$\mathrm{O} 2-\mathrm{Cu} 2-\mathrm{N} 9$ & $94.0(2)$ \\
$\mathrm{N} 2-\mathrm{Cu} 2-\mathrm{N} 9$ & $133.7(3)$ \\
$\mathrm{O} 2-\mathrm{Cu} 2-\mathrm{N} 8$ & $173.1(2)$ \\
N2-Cu2-N8 & $81.8(2)$ \\
N9-Cu2-N8 & $79.1(2)$ \\
$\mathrm{O} 2-\mathrm{Cu} 2-\mathrm{N} 11$ & $101.7(2)$ \\
N2-Cu2-N11 & $112.2(2)$ \\
N9-Cu2-N11 & $105.3(3)$ \\
N8-Cu2-N11 & $79.7(2)$ \\
C50-O1-Cu1 & $146.3(5)$ \\
C50-O2-Cu2 & $144.0(4)$ \\
N2-N1-Cu1 & $133.9(4)$ \\
C2-N1-Cu1 & $115.4(5)$ \\
N1-N2-Cu2 & $135.3(4)$ \\
C3-N2-Cu2 & $115.4(5)$ \\
C16-N3-Cu1 & $104.3(4)$ \\
C5-N3-Cu1 & $109.1(4)$ \\
C4-N3-Cu1 & $107.8(4)$ \\
C8-N4-Cu1 & $142.4(5)$ \\
C6-N4-Cu1 & $110.9(5)$ \\
\hline &
\end{tabular}





\section{Abbreviations}

$\begin{array}{ll}\text { Bu } & \text { (tert)-butyl } \\ \text { ATR } & \text { attenuated total reflection } \\ \text { Bz } & \text { benzyl } \\ \text { DBED } & \text { Di-tert-buthyl ethylene diamine } \\ \text { DBP } & \text { 3.5-di-tert-buthylphenol } \\ \text { DCM } & \text { dichloromethane } \\ \text { DHP } & \text { 3,4-dihydropyran } \\ \text { DME } & \text { dimethoxyethane } \\ \text { DMF } & \text { N,N-dimethylformamide } \\ \text { DMP } & 2,6 \text {-dimethylphenol } \\ \text { DPQ } & 3,3 \text { ',5,5'-tetramethyl-4,4'-diphenoquinone } \\ \text { DTBP } & 2,4 \text {-di-tertbutylphenol } \\ \text { DTBQ } & 3,5 \text {-di-tertbutyl quinone } \\ \text { EPR } & \text { electron paramagnetic resonance } \\ \text { ESI } & \text { electrospray ionization } \\ \text { Et } & \text { ethyl } \\ \text { GC-MS } & \text { gas chromatography-mass spectrometry } \\ \text { HDVV } & \text { Heisenberg-Dirac-Van-Vleck } \\ \text { HR } & \text { high resolution } \\ \text { HS } & \text { High spin } \\ \text { Im } & \text { Imidazol } \\ \text { iPr } & \text { isopropyl } \\ \text { IR } & \text { infra-red } \\ \text { LMCT } & \text { ligand-to-metal charge transfer } \\ \text { Me } & \text { methyl } \\ \text { MS } & \text { mass spectrometry } \\ \text { MTBE } & \text { methyl tert-butyl ether } \\ \text { NMR } & \text { nuclear magnetic resonance spctroscopy } \\ \text { OTf } & \text { triflate } \\ \text { Ph } & \text { Phenyl } \\ \text { pMMO } & \text { particulate methane monooxygenase } \\ \text { ppm } & \text { parts per million } \\ \text { pyrazole } \\ \text { room temperature } \\ \text { residue } \\ \text { DE }\end{array}$


sMMO soluble methane monooxygenase

SQUID superconducting quantum interference device

TBA tetrabutylammonium

tetb rac-5,5,7,12,12,14-hexamethyl-1,4,8,11,tetraazacyclotetradeca-4,11-diene

THF tetrahydrofuran

THP tetrahydropyran

TMBB 4,4'-dihydroxy-3,3',5,5'tetramethyl bibenzyl

tmeda tetramethyl ethylen diamine

TMP 2,4,6-trimethylphenol

TMS trimethylsilyl

TMSQ 3,3',5,5'-tetramethylstilbene-4,4'-quinone

TON turn-over number

Tp tris(pyrazolyl)borate

TPA tris(2-pyridylmethyl)amine

tren tris(2-aminoethyl)amine

Ts tosyl

UV ultraviolet

vis visible 


\section{MARTEN SEEBA}

Georg August Universität Göttingen $\diamond$ Tammannstraße $4 \diamond 37077$ Göttingen $(+49) 5513910638 \diamond$ marten.seeba@chemie.uni-goettingen.de

\section{EDUCATION}

Georg August Universität Göttingen

B.Sc. Chemistry

$10 / 2007-9 / 2010$

M.Sc. Chemistry

$10 / 2010-10 / 2012$

Ph.D. candidate in the CaSuS (Catalysis for Sustainable Synthesis)

program under the supervision of Prof. Dr. Franc Meyer

$01 / 2013-11 / 2013$

\section{DISSERTATION}

\section{Bioinspired dinuclear copper complexes for oxidation catalysis}

Inspired by tyrosinase, a type-III copper protein, that is capable of oxygenating and oxidizing phenolic substrates, several dinuclear copper complexes based on a pyrazolate bridged, dinucleating scaffold are synthesized. These complexes are investigated towards their structural properties and their reactivity with respect to to oxidation of phenolic substrates, as well as their ability to activate dioxygen.

\section{TEACHING}

- Supervision of one Bachelor student and two laboratory students

- Supervision of basic and advanced laboratory courses for chemistry and biochemistry students: Advanced Inorganic Synthesis, Methods in homogeneous and heterogeneous catalysis, Biological Chemistry, Principles in Chemistry and Laboratory Work

- Seminars for non-chemists explaining fundamentals and principles of chemistry

\section{EXPERIENCE}

Departamento de química orgánica, Universidad de Vigo, Spain

$09 / 2011-03 / 2012$

- Erasmus stay and practical laboratory course in the working group of Prof. Dr. Carmen Terán Moldes

Dipartimento di chimica, Università degli Studi di Pavia, Italy

$03 / 2013$ and $09 / 2014$

- Scientific exchange in the framework of the Cost Action CM 1003 "Biological Oxidation Reactions". The work done in the group of Prof. Dr. Luigi Casella will result in a joint publication. The manusscript is in preparation.

\section{Fakultät für Chemie, Georg August Universität Göttingen, Germany}

- Elected member of student representatives

2008-2011

- Participation in student and academic self-government

- Assistant job for restructuring and combining of study and test regulation into a new unified study regulation

- Member of the student based organizing committee for the "International Summer School on Green Chemistry", Göttingen 2011

\section{Experimental Techniques}

- Standard chracterization techniques: NMR, IR, UV/Vis, resonance Raman, mass spectrometry, GC/MS, HPLC, EPR 
- handling air and moisture sensitive compounds under inert conditions using Schlenk-technique and a glove-box setup

- Kinetic measurements using (cryo) stop flow setups for UV/Vis and IR measurements and maintenance of these instruments

- Polymer analysis via NMR, Gel-permeation Chromatography (GPC) and thermogravimetric analysis coupled with differential scanning calometry (TG/DSC)

\section{Method Courses}

- Kinetics in catalysis (Dr. Martin Klußmann, MPI Mühlheim)

- Spectroscopic methods in catalysis research (Prof. Dr. Christophe Copéret, ETH Zürich)

- Spectroscopic methods in heterogeneous catalysis (Prof. Angelika Brückner, LIKAT Rostock)

- Computational methods in catalysis research (Prof. Ricardo Mata, University Göttingen)

\section{Conferences attended}

· Niedersächsisches Katalysesymposium (NiKas), Göttingen, Germany 2014

- CaSuS Symposium at LIKAT (Leibniz Institut für Katalyse), Rostock, Germany 2014

- COST Action Meeting and IRTG 1422 Symposium, Lund, Sweden 2014

- IRTG 1422 Final Symposium, Göttingen, Germany 2015

- ICASEC (International center for advanced studies of energy conversion) Kick-off meeting, Göttingen, Germany 2015

- EuroBIC, Budapest, Hungary 2016

- Niedersächsisches Katalysesymposium (NiKas), Braunschweig, Germany, 2016.

\section{Languages}

- German (native)

· English (fluent, TOEFL iBT 112 Points, January 2013)

- Spanish (fluent, self-taught, no certificate)

- Italian (basic knowledge) 


\section{Acknowledgement}

First of all I would like to thank Prof. Franc Meyer, for the opportunity to work on this challenging project, for his continuous support, for providing an excellent working atmosphere, and for the scientific freedom he has granted me during the past years. I would also like to thank Prof. Sven Schneider for co-supervising this thesis.

I am very thankful to Prof. Luigi Casella for the opportunity to spend two months in his group during which I learned a lot, and for his continuous support and encouragement.I am also thankful to Dr. Enrico Monzani, who always had an open ear and taught me a lot during my stay. I would also like to thank everybody in LabCas for welcoming me to the group and to Pavia.

I am thankful to Dr. Sebastian Dechert for measuring crystal structures. I would also like to thank him for his support with all computer related questions and doing an excellent job as president and only steady member of the AK Meyer coffee club.

I would like to thank Dr. Claudia Stückl for measuring EPR samples, for many useful discussions, and her continuous enthusiasm.

I would like to thank Dr. Michael John, Ralf Schöne and the whole NMR service department.

I am thankful to Dr. Serhiy Demeshko for SQUID measurements.

I would like to thank Frank Hambloch for teaching me how to operate GC/MS and HPLC instruments and his continuos support in technical questions and problems.

I want to thank Alex Gers-Barlag, Mike Schütze, as well as Dr. Fraundorf and and the mass spectrometry department for ESI and MALDI measurements and I also want to thank the glass service, chemical storage service, and all care takers.

I would also like to thank my labmates Jörg Teichgräber, Nicole Kindermann, Vandana Goswami and Jerome Günes for the great atmosphere in the lab, and for drying solvents and sharing chemicals.

I would like to offer my grattitude to Kristian Dalle and Alexander Brinkmeyer 
for fruitful discussions and moral support.

I would like to thank Dr. Claudia Stückl, Britta Müller, and Petra Unger for taking care of all administrative issues.

I would like to thank Joanne Wong, Alexander Brinkmeyer, Marie Bergner, Mike Schütze and Hendrik Stevens for thoroughly proof-reading my thesis. I want to thank everybody in AK Meyer for a wonderful time in and out of the lab, and for being colleagues and friends.

Finally, I want to thank my family, for their outstanding support throughout the years that hepled me get to where I am now. 